\title{
EFFECTS OF DAMAGE ARRESTMENT DEVICES ON THE FATIGUE LIFE OF IMPACTED COMPOSITE SANDWICH BEAMS
}

\author{
A Thesis \\ presented to \\ the Faculty of California Polytechnic State University, \\ San Luis Obispo
}

\author{
In Partial Fulfillment \\ of the Requirements for the Degree of \\ Masters of Science in Aerospace Engineering
}

By

Yvette Vanessa Wood

March 2013 
(c) 2013

Yvette Vanessa Wood

ALL RIGHTS RESERVED 
TITLE:

AUTHOR:

DATE SUBMITTED:

COMMITTEE CHAIR:

COMMITTEE MEMBER:

COMMITTEE MEMBER:

COMMITTEE MEMBER:
Effects of Damage Arrestment Devices on the Fatigue Life of Impacted Composite Sandwich Beams

Yvette Vanessa Wood

March 2013

Faysal Kolkailah, Ph.D, P.E.

Eltahry Elghandour, Ph.D

Eric Kasper, Ph.D, P.E.

Susan Opava, Ph.D 


\section{Abstract}

Effects of Damage Arrestment Devices on the Fatigue Life of Impacted Composite Sandwich

\section{Beams}

Yvette Vanessa Wood

The use of composite sandwich panels is rapidly increasing in the aerospace industry. In these applications, thin composite face sheets are typically co-cured to foam or honeycomb cores. One of the greatest concerns in these types of applications is face-core delamination because it can ultimately lead to catastrophic failure of the structure. Further, the effects of foreign object damage on composite sandwich structures are often catastrophic. Damage to the structure is often undetectable through visual inspection, leading to concerns of unknown interlaminar damage through the life of the structure, which can cause a catastrophic failure. This research determines the effectiveness of a damage arrestment device (DAD) on the structural integrity of composite sandwich beams. The effects of these DADs on the fatigue life of these beams following a low-velocity impact will be considered.

Composite sandwich beams were constructed utilizing two layers of Advanced Composite Group LTM45EL/CF1803 bi-directional woven carbon fiber face sheets with a General Plastics Last-A-Foam FR-6710 rigid polyurethane core, and were cured utilizing an autoclave. Static 4-point bend testing following the ASTM standard D6272 was investigated to determine the flexural behavior of composite sandwich beams. Six different beam cases, with dimensions 1-inch wide by 11.75 -inch long, were investigated. The cases considered were beams with and without initial delamination and also, with DAD keys placed transversely and longitudinally with and without delamination. A drop test was employed to simulate a low-velocity impact to the composite sandwich beams with and without delamination. The flexural behavior of these two cases was determined and used in fatigue testing. Experimental results showed that the addition 
of DAD keys to initially delaminated composite sandwich beams improved the ultimate strength values significantly.

Fatigue testing was investigated to determine the fatigue life of composite sandwich beams following a low velocity impact. Similar to static 4-point bend testing, six different beam cases, with dimensions 1 -inch wide by 11.75 -inch long, were investigated. The cases considered were exactly those seen in static 4-point bend testing - beams with and without initial delamination and also with DAD keys placed transversely and longitudinally with and without delamination. The three cases without delamination were investigated in the 4-point bend fatigue test, with and without impact. Experimental results showed that the addition of DAD keys had a significant effect on increasing the fatigue life of composite sandwich beams with initial damage from impact, and even improved the fatigue life of beams with initial delamination.

A numerical investigation was completed using a finite element model (FEM) as a means to validate the experimental results found in this research. Static 4-point bend testing was modeled using Abaqus, where maximum deflection values at a specific load were used as a comparison between the experimental and numerical results. Experimental and numerical results showed that the addition of DADs significantly improved the integrity and fatigue life of the composite sandwich design. All cases showed close agreement between the experimental and numerical results. 


\section{Acknowledgement}

I would like to take this opportunity to express my deepest gratitude and appreciation towards my advisers Dr. Faysal Kolkailah and Dr. Eltahry Elghandour for guiding me through my undergraduate and graduate studies, and my research. Both took the time to offer their support and expertise during this research and for that I am very grateful.

In addition, I would like to thank the California Central Coast Research Partnership $\left(\mathrm{C}^{3} \mathrm{RP}\right)$ and the Cal Poly Aerospace Engineering department for funding this research. This research was sponsored by the Department of the Navy, Office of Naval Research, under Award \# N00014-11-1-0359. Further, I would like to thank my thesis committee members, Dr. Susan Opava and Dr. Eric Kasper, for their assistance in reviewing my paper and for taking the time to provide me with feedback. I give many thanks to Cody Thompson for his constant support; he was always willing to help when needed in the lab. I would also like to thank my fellow peers for their help in the lab, especially Kodi Rider for developing and building a data acquisition (DAQ) system for the Dynatup 8250 drop weight impact tester and Daniel Barath for designing and manufacturing the impact test support fixture and impacting tup.

Lastly, I would like to thank my parents for their support through my life, especially through my studies. When I expressed my desire to continue my education my parents welcomed the idea and offered their support one hundred percent. Without their financial and emotional support this journey would not have been possible. Ultimately, I pushed through the toughest times for my late brother, Joey. He is the reason I have been successful because he has always guided me in the right direction and even though he is gone, his spirit still lives inside me, pushing me to be the best I can be. I dedicate my thesis to Joseph Patrick Wood, because he never had the opportunity. 


\section{Table of Contents}

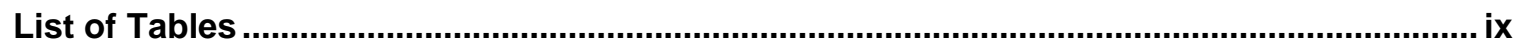

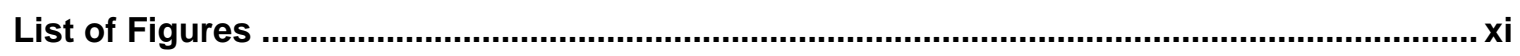

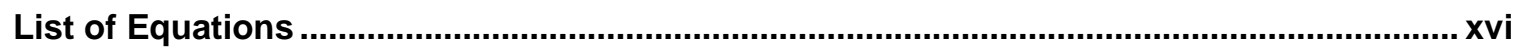

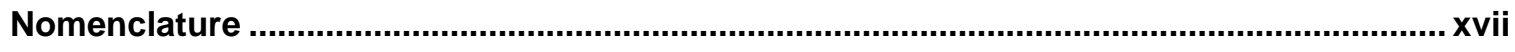

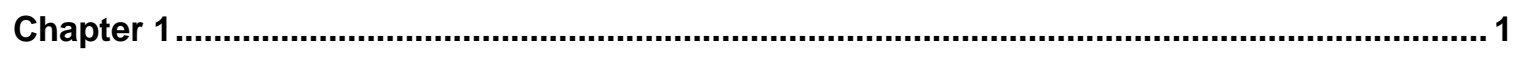

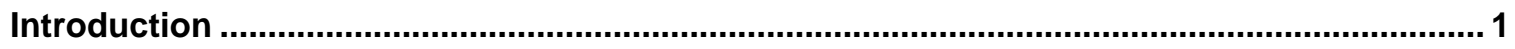

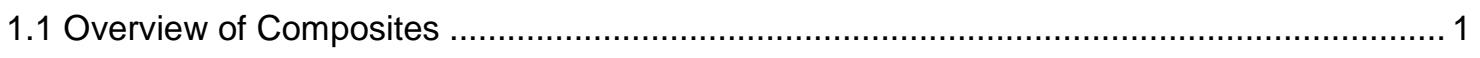

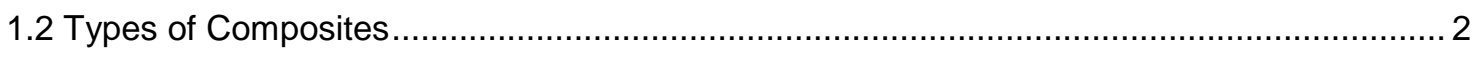

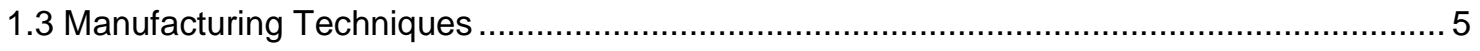

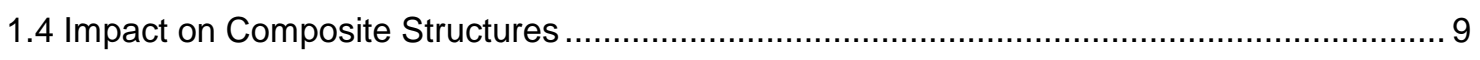

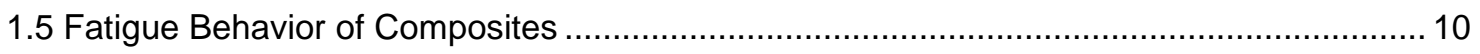

1.6 Previous Research in Fatigue of Composite Materials ...................................................... 12

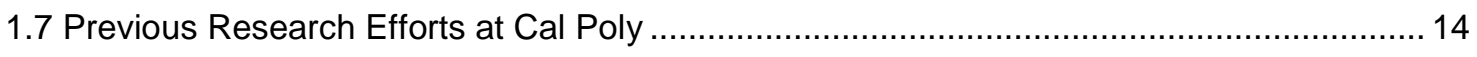

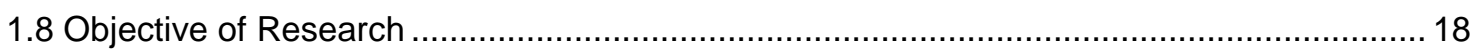

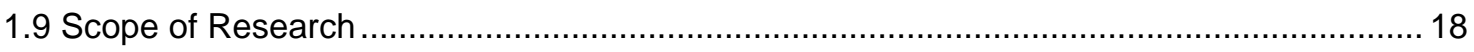

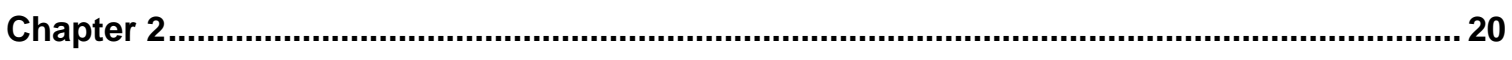

Specimen Manufacturing and Mechanical Characteristics ................................................. 20

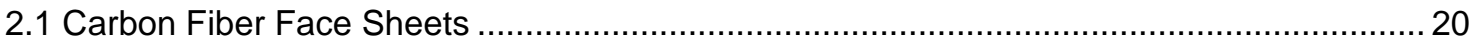

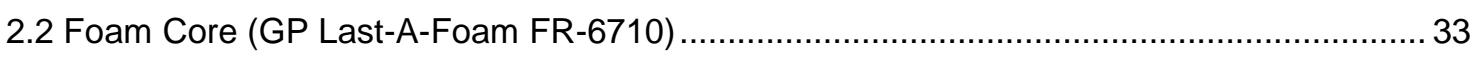

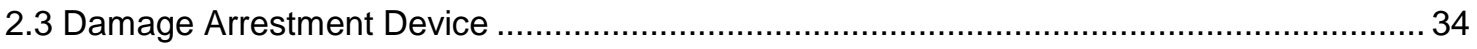

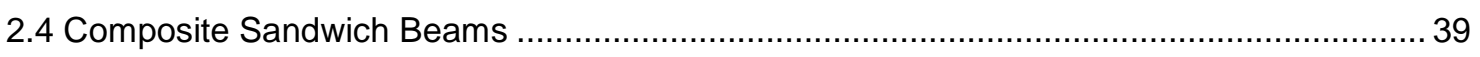

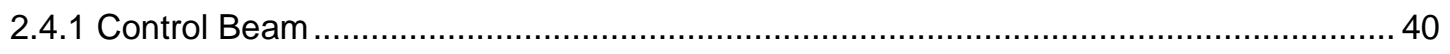

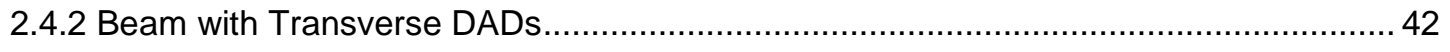

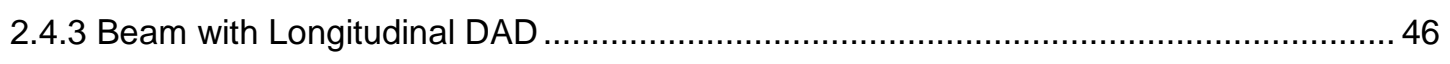

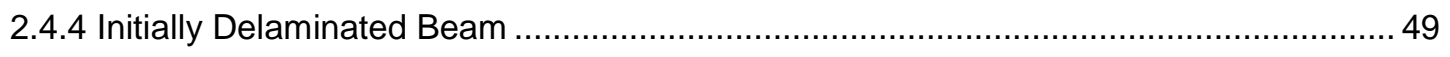

2.4.5 Initially Delaminated Beam with Transverse DADs …............................................ 51

2.4.6 Initially Delaminated Beam with Longitudinal DAD ................................................... 53

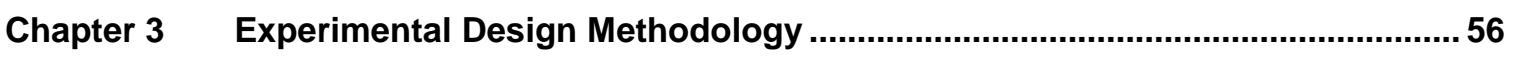

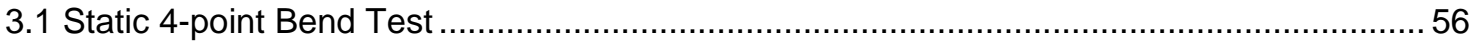

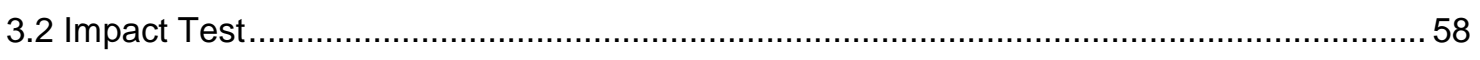

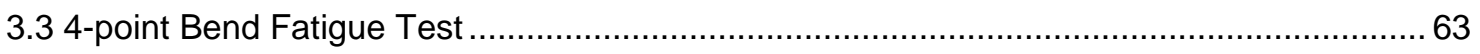

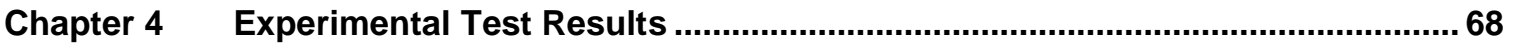




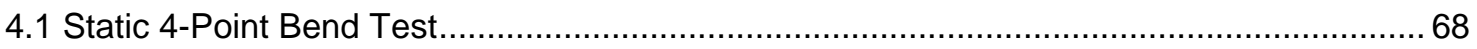

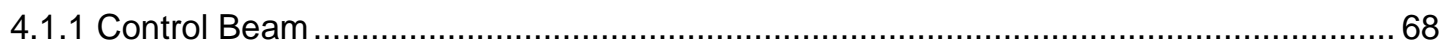

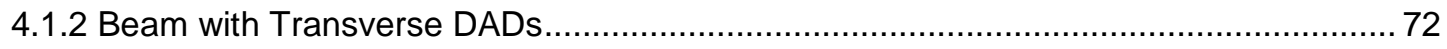

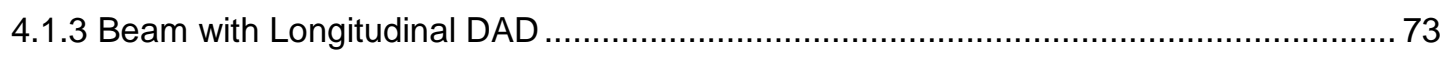

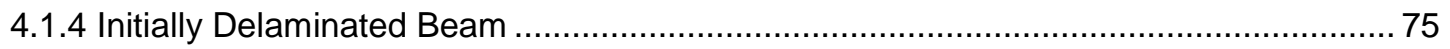

4.1.5 Initially Delaminated Beam with Transverse DADs ….............................................. 77

4.1.6 Initially Delaminated Beam with Longitudinal DADs.................................................... 79

4.1.7 Comparison of Composite Sandwich Beam Test Results ........................................... 81

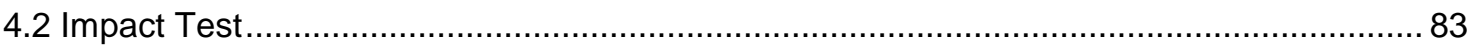

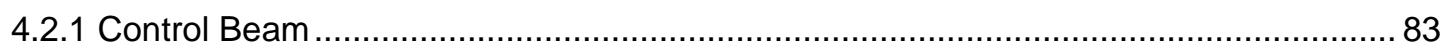

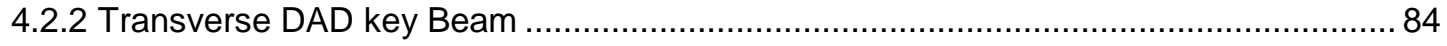

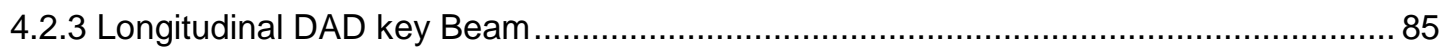

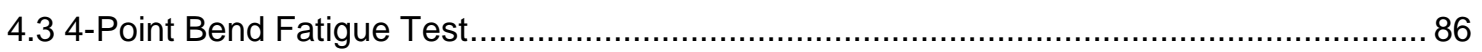

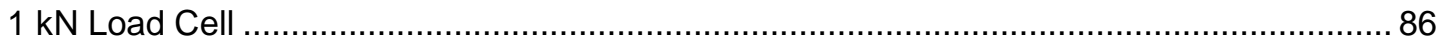

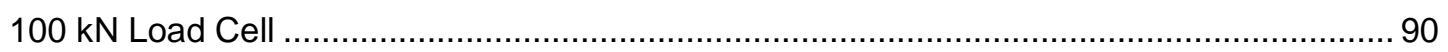

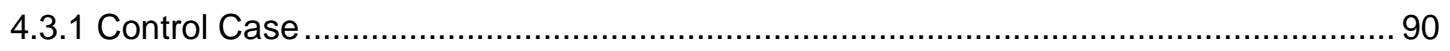

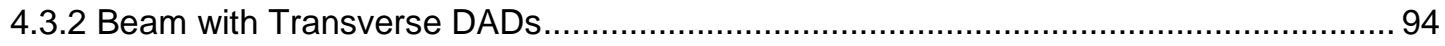

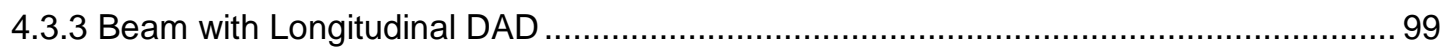

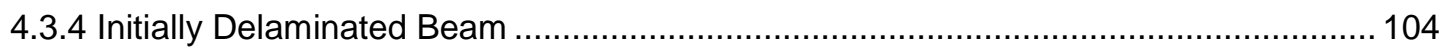

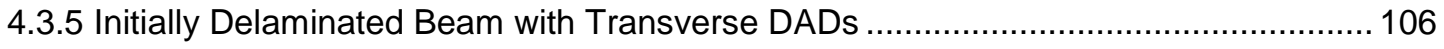

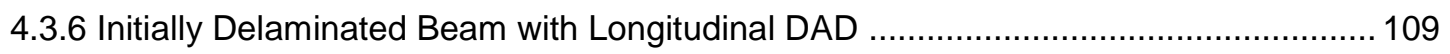

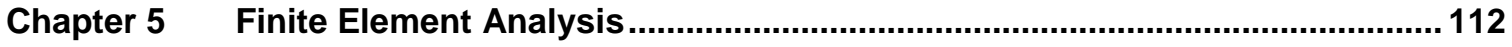

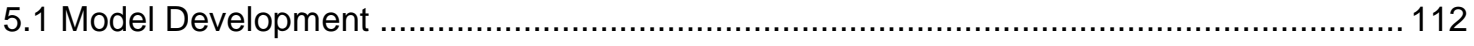

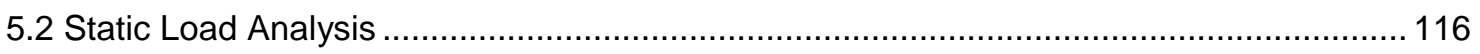

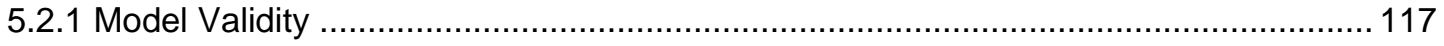

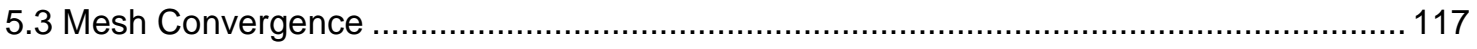

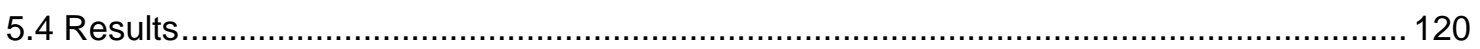

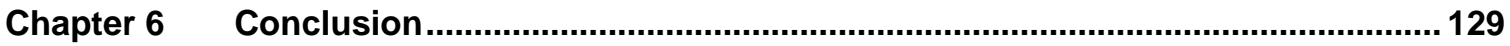

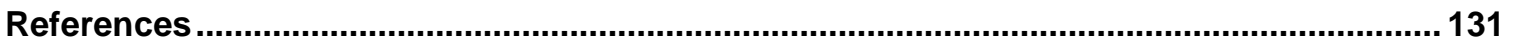

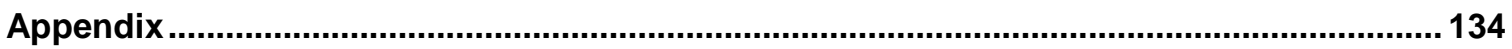




\section{List of Tables}

Table 2.1 Mechanical characteristics summary of LTM45EL/CF1803 carbon fiber.

Table 2.2 Mechanical properties of PVC foam [21]

Table 2.3 Mechanical properties of fiberglass damage arrestment device [22]

Table 4.1. Results from experimentally investigated static 4-point bend testing for all test cases.

Table 4.2. Fatigue test results of control test beams using $1 \mathrm{kN}$ load cell.

Table 4.3. Fatigue test results of control test beams using $100 \mathrm{kN}$ load cell.

Table 4.4. Fatigue test results of impacted control test beams using $100 \mathrm{kN}$ load cell.

Table 4.5. Fatigue test results of test beams with transverse DAD keys and no delamination.

Table 4.6. Fatigue test results of impacted test beams with transverse DAD keys using $100 \mathrm{kN}$ load cell.

Table 4.7. Fatigue test results of longitudinal DAD key beams with no delamination.

Table 4.8. Fatigue test results of impacted test beams with Longitudinal DAD keys using $100 \mathrm{kN}$ load cell. 102

Table 4.9. Fatigue test results of test beams with one inch initial delamination.

Table 4.10. Fatigue test results of test beams with one inch initial delamination and transverse DAD keys.

Table 4.11. Fatigue test results of test beams with one inch initial delamination and Longitudinal DAD key.

Table. 5.1. FE Simulation material properties for composite sandwich beam.

Table 5.2. FE model and hand calculation comparison

Table 5.3. Results for four-point bending condition on non-delaminated control beam.

Table 5.4. Results for four-point bending condition on delaminated beam.

Table 5.5. Results for four-point bending condition on non-delaminated beam with transverse DADs.

Table 5.6. Results for four-point bending condition on delaminated beam with transverse DADs.

Table 5.7. Results for four-point bending condition on non-delaminated beam with Transverse DADs. 
Table 5.8. Results for four-point bending condition on delaminated beam

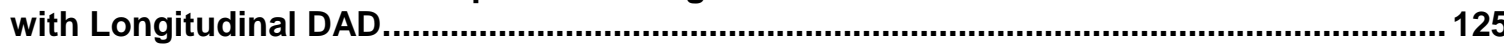

Table 5.9. Comparison of all Numerical and Experimental Deflections............................... 126 


\section{List of Figures}

Figure 1.1. Microstructure of a particulate composite material. [3]....................................... 3

Figure 1.2. Unidirectional fibrous composite.[4] .............................................................. 3

Figure 1.3 Schematic of honeycomb composite sandwich panel. [6] ................................... 5

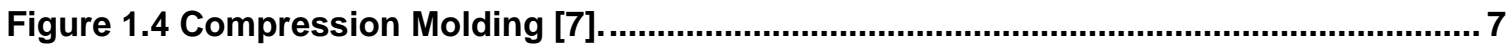

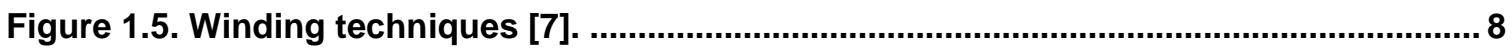

Figure 1.6. F-111 nose cone after bird-strike. [8]

Figure 1.7. The development of damage in composite laminates. [10]................................ 12

Figure 1.8. Circular DADs used in Richard Balabat's thesis [17]. .......................................... 15

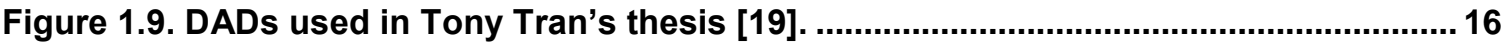

Figure 2.1. LTM45EL / CF1803 bidirectional woven carbon fiber ......................................... 21

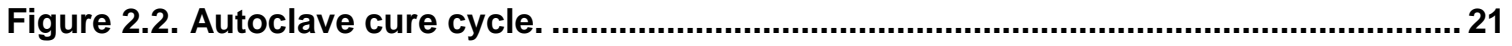

Figure 2.3. Schematic of layup and materials used to cure face sheets in

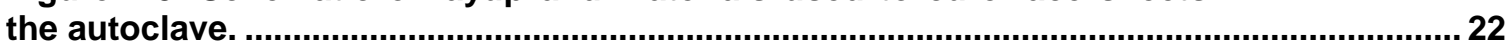

Figure 2.4. Lay-up Stacking Pattern of Materials for cure in the autoclave.......................... 22

Figure 2.5. Carbon fiber face sheet lay-up placed inside autoclave cure

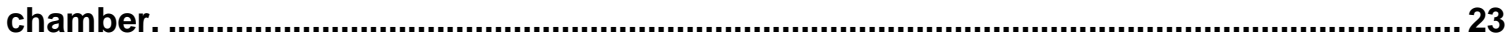

Figure 2.6. Wet tile saw used to cut test coupons for ASTM standard D3039...................... 24

Figure 2.7. Test coupons cut to be tested according to ASTM standard

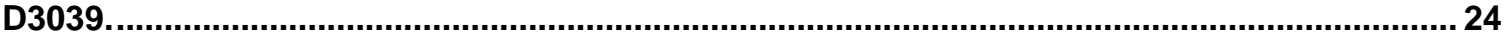

Figure 2.8. Prepared carbon fiber Test coupon ready for testing......................................... 25

Figure 2.9. Carbon fiber test coupon secured in the Instron 8801 grips. .............................. 26

Figure 2.10. Typical failure mode of the carbon fiber test coupon. ....................................... 26

Figure 2.11. Tensile stress-strain curves obtained by experiment for face

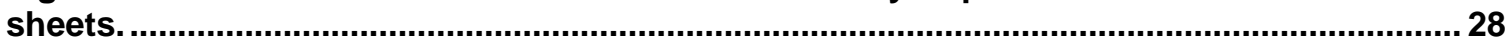

Figure 2.12. Possible strain gage locations on carbon fiber test coupons. ........................... 28

Figure 2.13. Plot of average strains versus applied load for determination of

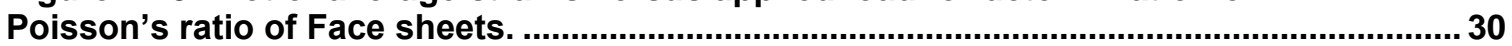

Figure 2.14. Plate used in experiment on a digital specimen weighing scale. ....................... 31

Figure 2.15. Oven used in experiment to complete resin burn-off procedure......................... 32

Figure 2.16. Woven roving fiberglass separated into unidirectional

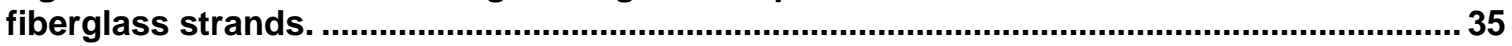

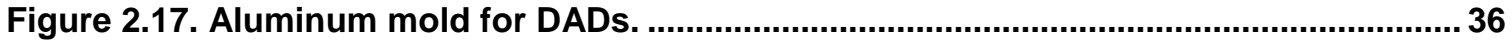

Figure 2.18. Mold release wax used to separate curved fiberglass from mold.

Figure 2.19. Bundles composed of 15 strands of unidirectional fiberglass. 
Figure 2.20. Hand Lay-up Method Used to Fabricate DAD Keys.

Figure 2.21. 200-lb of weight used to compress fiberglass DADs into mold.......................... 38

Figure 2.22. DADs are sanded down until separated from aluminum mold ........................... 38

Figure 2.23. Cured DADs separated from the aluminum mold ........................................39

Figure 2.24. Layup schematic of composite sandwich plate for curing in the autoclave.

Figure 2.25. Drawing of a control case beam with no DAD keys or initial delamination.

Figure 2.26. Epoxy resin/hardener applied to DADs used for secure bond in foam.

Figure 2.27. Milled foam sheet with DADs placed transversely (dimensions in inches).

Figure 2.28. Layup sequence of composite sandwich plate with transverse keys ready for curing in the autoclave.

Figure 2.29. plate with no initial delamination and transverse DADs placed in the autoclave with tight vacuum.

Figure 2.30. Drawing of a beam with transverse DAD keys and no initial delamination.

Figure 2.31. CNC machine milling slots in polyurethane foam to accept DAD keys.

Figure 2.32. Milled foam sheet with DADs placed longitudinally.

Figure 2.33. Layup sequence of composite sandwich plate with longitudinal keys ready for curing in the autoclave.

Figure 2.34. Cured composite sandwich plate with no initial delamination and longitudinal keys.

Figure 2.35. Configuration of beam with longitudinal DAD key and no delamination.

Figure 2.36. Drawing of a beam with longitudinal DAD key and no initial delamination.

Figure 2.37. Foam core with sketched delamination placement (left) and non-porous strip placed centrally (right).

Figure 2.38. Layup of composite sandwich plate with initial delamination and sealant tape placement for curing in the autoclave. 50

Figure 2.39. Drawing of a beam with initial delamination and no DADs.

Figure 2.40. pre-cut foam sheet with DADs placed transversely and nonporous strip to force a delamination (dimensions in inches).

Figure 2.41. Test specimen beams with DADs placed transversely and initial delamination cut from cured plates.

Figure 2.42. Exploded view of a beam with transverse DAD keys and a centrally located 1-inch initial delamination.

Figure 2.43. Pre-cut foam sheet with DADs placed longitudinally and added non-porous strip.

Figure 2.44. Layup sequence of composite sandwich plate with longitudinal keys and delamination ready for curing in the autoclave. 
Figure 2.45. Exploded view of beam with longitudinal DAD key and initial delamination.

Figure 3.1. Schematic of 4-point bending test specifications followed by ASTM 6272 standard test [27].

Figure 3.2. Instron 8801 Servohydraulic Fatigue Testing System used for 4point bend testing.

Figure 3.3. Dynatup 8250 drop weight impact machine used for impact testing. 59

Figure 3.4. Test support fixture used for impact tests.

Figure 3.5. Two added support plates for clamping test beams in support fixture.

Figure 3.6. Dynatup DAQ software window with data acquisition in progress.

Figure 3.7. Screen shot of GUI with plots from impact test data.

Figure 3.9. Several drop test penetrations tested to choose ideal drop weight height for all test specimens.

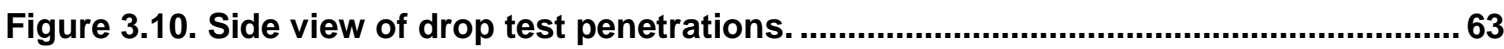

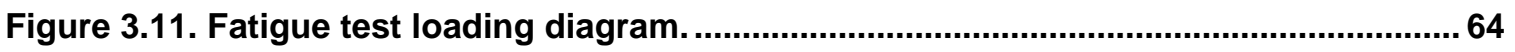

Figure 3.12. Test specimen beam secured in 4-point bend jig for 4-point bend fatigue testing.

Figure 3.13. Screen shot of Wavematrix plots during a dynamic 4-point bend test.

Figure 3.14. Small load cell used to perform 4-point bend fatigue testing on test specimen beams.

Figure 3.15. Control loop tuning tool for the Instron.

Figure 4.1. Sandwich beam load vs. deflection used to find mechanical properties.

Figure 4.2. Resulting flexural stress-strain curves of the control case beam specimens under 4-point bending.

Figure 4.3. Typical failure mode of control case beam subjected to static 4point bending.

Figure 4.4. Control case group failure mode nearly identical in each test specimen.

Figure 4.5. Final failure mode of composite sandwich beam test group with added transverse DAD keys.

Figure 4.6. Resulting flexural stress-strain curves of specimens with no initial delamination and added transverse DAD keys subjected to static 4point bending.

Figure 4.7. Final failure mode of composite sandwich beam test group with added longitudinal DAD keys and no initial delamination.

Figure 4.8. Resulting flexural stress-strain curves of composite sandwich specimens with no initial delamination and added longitudinal DAD keys subjected to static 4-point bending. .75

Figure 4.9. Typical failure mode of initially delaminated beams. 
Figure 4.10. Final failure mode of composite sandwich beam case with one inch initial delamination.

Figure 4.11. Resulting flexural stress-strain curves of test specimens with an initial one inch long delamination subjected to 4-point bending.

Figure 4.12. Final failure mode of composite sandwich beam with one inch initial delamination and added transverse DAD keys.

Figure 4.13. Final failure mode of composite sandwich beam test group with one inch initial delamination and added transverse DAD keys.

Figure 4.14. Resulting flexural stress-strain curves of composite sandwich beams with an initial one inch long delamination and transverse DAD keys subjected to static 4-point bending.

Figure 4.15. Final failure mode of composite sandwich beam with one inch initial delamination and added longitudinal DAD key.

Figure 4.16. Final failure mode of composite sandwich beam test group with one inch initial delamination and added longitudinal DAD key.

Figure 4.17. Resulting flexural stress-strain curves of longitudinal DAD key case group with an initial one inch long delamination subjected to static 4point bending.

Figure 4.18. beams with no initial delamination or DAD key Post-impact.

Figure 4.19. Composite sandwich beams with transverse DAD keys and no initial delamination Post-impact.

Figure 4.20. Composite sandwich beams with Longitudinal DAD key and no initial delamination Post-impact.

Figure 4.21. 1-kN load cell used for 4-point bend fatigue testing.

Figure 4.22. Control test specimen secured in 4-point bend jig for dynamic loading test.

Figure 4.23. Typical failure from fatigue test on control beam with no initial delamination or DAD key.

Figure 4.24. Control test group after final failure and completed fatigue life.

Figure 4.25. Typical failure from fatigue test at $1 \mathrm{~Hz}$ on control beam with no initial delamination or DAD key.

Figure 4.26. Control test group, tested with $100 \mathrm{kN}$ load cell, after final failure and completed fatigue life.

Figure 4.27. Typical failure from fatigue test at $1 \mathrm{~Hz}$ on previously impacted control beam with no initial delamination or DAD key.

Figure 4.28. Impacted Control test group tested with $100 \mathrm{kN}$ load cell, after final failure and completed fatigue life.

Figure 4.29. Typical failure from fatigue test at $1 \mathrm{~Hz}$ on beams with no initial delamination and transverse DAD keys.

Figure 4.30. Test group with the addition of transverse DAD keys after final failure and completed fatigue life.

Figure 4.31. Typical failure from fatigue test at $1 \mathrm{~Hz}$ on previously impacted beams with no initial delamination and transverse DAD keys.

Figure 4.32. Impacted test group with added transverse DAD keys tested with $100 \mathrm{kN}$ load cell, after final failure and completed fatigue life. 
Figure 4.33. Typical failure seen in fatigue test at $1 \mathrm{~Hz}$ in beams with no initial delamination and longitudinal DAD key.

Figure 4.34. Test group with the addition of a longitudinal DAD key after

final failure and completed fatigue life.

Figure 4.35. Typical failure from fatigue test at $1 \mathrm{~Hz}$ on impacted beam with no initial delamination and longitudinal DAD key.

Figure 4.36. Impacted beams with added Longitudinal DAD keys after final

failure and completed fatigue life.

Figure 4.37. Typical failure from fatigue test at $1 \mathrm{~Hz}$ on beams with an initial delamination.

Figure 4.38. Position trend of delaminated beam with transverse DAD keys.

Figure 4.39. premature failure from fatigue test at $1 \mathrm{~Hz}$ on control beam with initial delamination and transverse DAD key.

Figure 4.40. Position trend of delaminated beam with Longitudinal DAD

key.

Figure 4.41. Premature failure seen from fatigue test at $1 \mathrm{~Hz}$ on beam with initial delamination and longitudinal DAD key.

Figure 5.1. Composite Sandwich control Beam model.

Figure 5.2. Non-Delaminated Beam with Transverse DADs. .................................................113

Figure 5.3. Non-Delaminated Beam with Longitudinal DAD............................................113

Figure 5.4. Non-Delaminated Sandwich Beam with transverse DADs

Exploded View (inches).

Figure 5.5. Four-Point Bending Loading Condition, Two 50-Ib loads................................114

Figure 5.6. Location of RP for Convergence Study............................................................. 118

Figure 5.7. Convergence Study of Max Deflection. ............................................................ 119

Figure 5.8. Convergence Study of Stress at RP.

Figure 5.9. Displacement contour plot of control beam with deformation

factor $=1$.

Figure 5.10. Displacement contour plot of delaminated beam case with deformation factor $=1$.

Figure 5.11. Displacement contour plot of transverse DAD key case with deformation factor $=1$.

Figure 5.12. Displacement contour plot of delaminated beam with transverse dad keys with deformation factor $=1$.

Figure 5.13. Displacement contour plot of longitudinal DAD key case with deformation factor $=1$.

Figure 5.14. Displacement contour plot of delaminated beam with longitudinal DAD key with deformation factor $=1$. 


\section{List of Equations}

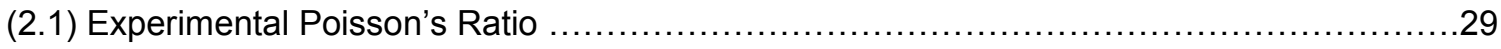

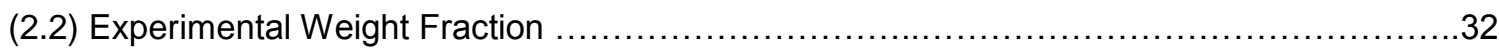

(3.1) Crosshead Loading Rate for Experimental 4-Point Bending .............................57

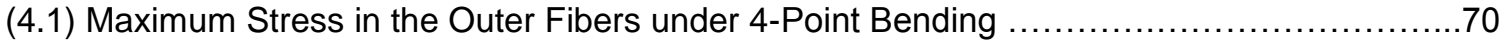

(4.2) Maximum Strain in the Outer Fibers under 4-Point Bending …........................70

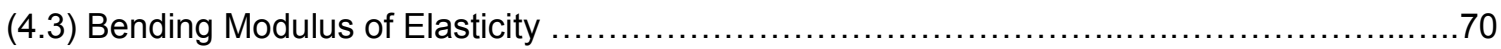




\section{Nomenclature}

D - flexural rigidity

Units

$\mathrm{E}$ - elastic modulus

$\mathrm{Ib}^{*} \mathrm{in}^{2}$

$F$ - force

psi

$G$ - shear modulus

$\mathrm{lb}$

I - area moment of inertia

$\mathrm{L}$ - length

$\mathrm{P}$ - load

$\mathrm{R}$ - crosshead loading rate

$T$ - time

$\mathrm{V}$ - volume fraction

$\mathrm{W}$ - weight fraction

$Z$ - minimum outer fiber straining rate

psi

in $^{4}$

in

lb

in $/$ min

$\mathrm{sec}$

$\%$

$\%$

in/in*min

a - acceleration

$\mathrm{ft} / \mathrm{s}^{2}$

$\mathrm{c}-$ loading width

in

$\mathrm{b}-$ width

in

d-depth

in

I - longitudinal

$\mathrm{m}$ - slope of the tangent to the initial straight line

$\mathrm{t}-$ face thickness

w - deflection

ä - displacement

in

å - strain

in/in

í - Poisson's ratio

n - density

$\mathrm{lb} / \mathrm{in}^{3}$

ó - stress

psi

\section{SUBCRIPTS}

$\mathrm{f}$ - failure

max - maximum

ult- ultimate

m- mean 


\section{Chapter 1}

\section{Introduction}

\subsection{Overview of Composites}

Composites have a long history of usage and although their precise beginnings are unknown, all recorded history contains references to some use of composite material. Some of the early uses of composites are found by Israelites who used straw to strengthen mud bricks. This provided reinforcement to buildings to maintain its strength and integrity. Further, plywood was used by ancient Egyptians when they realized that wood could be rearranged to achieve higher strength and resistance to thermal expansion and swelling. Later, the Mongols invented the first composite bow, which used a combination of wood, bone, and animal glue. In combination, these materials provided an extremely powerful and accurate weapon [1]. More recently, composite materials have become important in everyday living, for example, the use of concrete, which is typically composed of crushed rocks, sand, and water.

The word composite in the term composite material signifies two or more materials that are combined on a macroscopic scale to form a valuable third material. Many materials can be combined on a microscopic scale, such as the alloying of metals, but the resulting material is macroscopically homogeneous. A composite material, however, usually exhibits the best qualities of its constituents and typically some qualities that neither constituent possesses. Properties that can be improved include but are not limited to: strength, stiffness, fatigue life, weight, corrosion resistance, thermal insulation, and thermal conductivity [1]. However, these properties are not always improved at the same time and sometimes some of these properties can conflict with one another. The goal is to create a material that has improved characteristics necessary to perform the required design task. 
In various industries today, including the aerospace industry, composites have surpassed the use of conventional metallic materials such as aluminum and steel. The composites industry has grown so rapidly because of the exceptional mechanical properties, ease of fabrication, and the rare flexibility in design capabilities. Further, composites have advantages over traditional materials that include light weight, corrosion resistance, impact resistance and excellent fatigue strength [2]. With the continuous development of new composite materials, the composites industry has sustained its growth.

\subsection{Types of Composites}

There are four classes of composite materials: particulate, fibrous, laminated, and a combination of the first three types [1]. In general, each type of composite material listed is fabricated to improve mechanical properties such as toughness, strength, stiffness, and high temperature performance. The geometry of the reinforcement greatly determines the strength of the material. Thus, it is common to classify the material based on the geometry of a representative unit of reinforcement [2].

Particulate composites are a composite whose reinforcement is classified as particles. Typically, a particle has no long dimension and is nonfibrous, and thus, is not as effective in improving fracture resistance. However, particles are effective in enhancing the stiffness of a composite since particles share the load to a smaller extent than fibers in a fibrous composite. The materials used in a particulate composite material can be any combination of metallic and non-metallic materials. A microstructure of a particulate composite can be seen in fig. 1.1, where the dark areas represent the particulates. Particulate composites are advantageous in applications that require improved performance at elevated temperatures. Particles of tungsten, molybdenum, or other carbides in copper and silver matrices are used extensively for electricalcontact applications because of their ability to withstand high thermal and electrical conductivity conditions [2]. However, the most widely known particulate composite is concrete, where rocks and sand are considered the particles and the cement is considered the matrix material. 


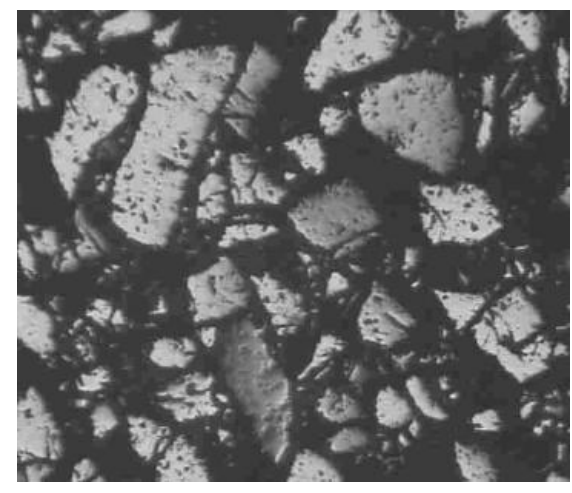

Figure 1.1. Microstructure of a particulate composite material. [3]

Fiber-reinforced composites are classified as a combination of small cross-sectional fibers and an embedded matrix. The matrix serves to bind the fibers together, transfer the loads to the fibers, and protect fibers from any type of environmental damage due to handling [2]. Figure 1.2 illustrates a typical fibrous composite material, where the fibers are unidirectional. Fibrous composites can be categorized by either continuous or discontinuous fiber-reinforced composites. Continuous-fiber-reinforced composites include long fibers, while discontinuousfiber-reinforced composites contain short fibers. Continuous fiber-reinforced composites are typically made by aligning short fibers through injection molding. Damage encountered during the loading of continuous fiber reinforced composites often involves brittle failure processes, where fibers are reloaded by the matrix via shear stress at the fiber-matrix interface. Discontinuous fiberreinforced composites are used when the stiffness and higher strength of continuous fiberreinforced composites are not required, since the manufacturing costs are much lower. Some of the more widely used fibrous composites include glass-fiber reinforced polymers (fiberglass) and carbon-fiber reinforced polymers. In general, fibrous composites are made of strong, brittle fibers in a more ductile matrix.

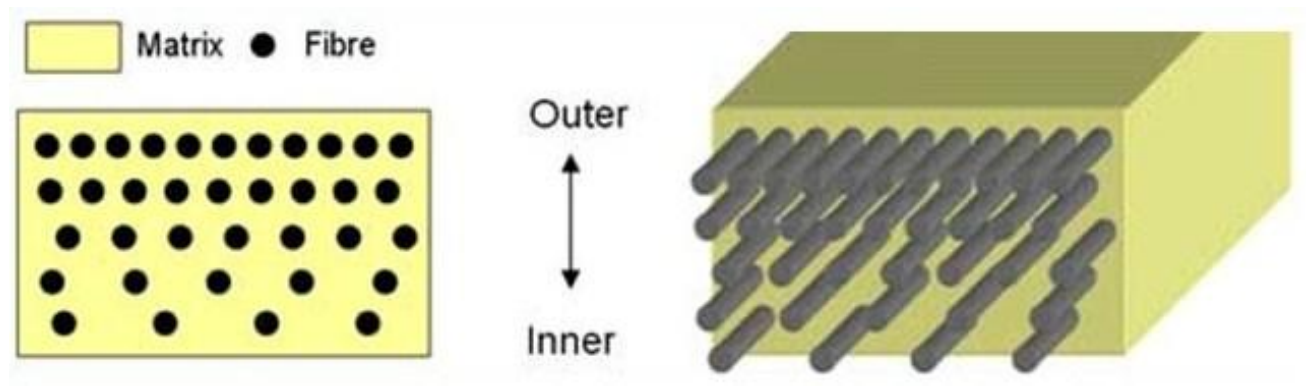

Figure 1.2. Unidirectional fibrous composite.[4] 
Laminate composites are a construction of layers of fibrous composite materials which can be uniquely oriented to provide required engineering properties, including strength, in-plane stiffness, bending stiffness, and thermal expansion. The individual layer consists of high-modulus, high-strength fibers in a metallic, polymeric, or ceramic matrix material [2]. Types of laminate composites include bimetals, clad metals, laminated glass, and plastic-based laminates. A laminate composed of two different metals combined is considered a bimetal, where the thermal expansion coefficient is significantly different. Typically, bimetals are used in high temperaturemeasuring devices because of their ability to warp and deflect a great amount. Clad metals are obtained by cladding or sheathing one metal with one another. A good example of a clad metal is a high strength metal alloy covered with a corrosion resistant material. When combined, the composite clad metal is both corrosion resistant and high in strength. Laminated glass follows a similar concept, where glass, a brittle material, is coated with another material to create a safe and transparent composite. This is seen widely in the automobile industry on windows and windshields of an automobile. Plastic-based laminates are materials that have been saturated with various plastics and are typically used for impact and penetration-resistant applications [2].

A composite sandwich core panel is a type of laminate composite that consists of two or more different materials that are combined to make a structure which is high in strength and low in weight. Typically, a low-density lightweight core material is sandwiched between two thin laminated face sheets, which dramatically increase a laminate's stiffness with little weight added. Without a core material, the thin face sheets would be forced to withstand all tensile and compressive loads in shear, torsion, and bending. With the core material bearing shear loads, the thin face sheets are left to withstand in-plane loads. Typical face sheet laminates include, fiberglass, carbon fiber, and aramid laminates. Although face sheet material plays a significant role in the overall structure, the core of the structure is the foundation of the overall strength. In aerospace applications, high performance aluminum and aramid honeycomb cores are used in aircraft primary structure, interior panels, and aircraft flooring. The composition of a honeycomb composite sandwich panel is illustrated in fig. 1.3. Honeycombs, foam, and balsa wood play a significant role in structural parts for the transportation, wind energy, and marine industries. A 
sandwich structure's bending stiffness is proportional to the core thickness, where doubling the core thickness yields a structure six times stronger and twelve times stiffer with little weight addition [5]. Further, the core helps distribute stresses and loads on the face sheets, which is excellent for impact. The compression strength of a core prevents the face sheets from wrinkling failure, which is a typical failure mode of a composite sandwich. Additional failure modes of composite sandwiches under static or dynamic loading include face-core delamination, core failure, impact failure, or failure due to compression.

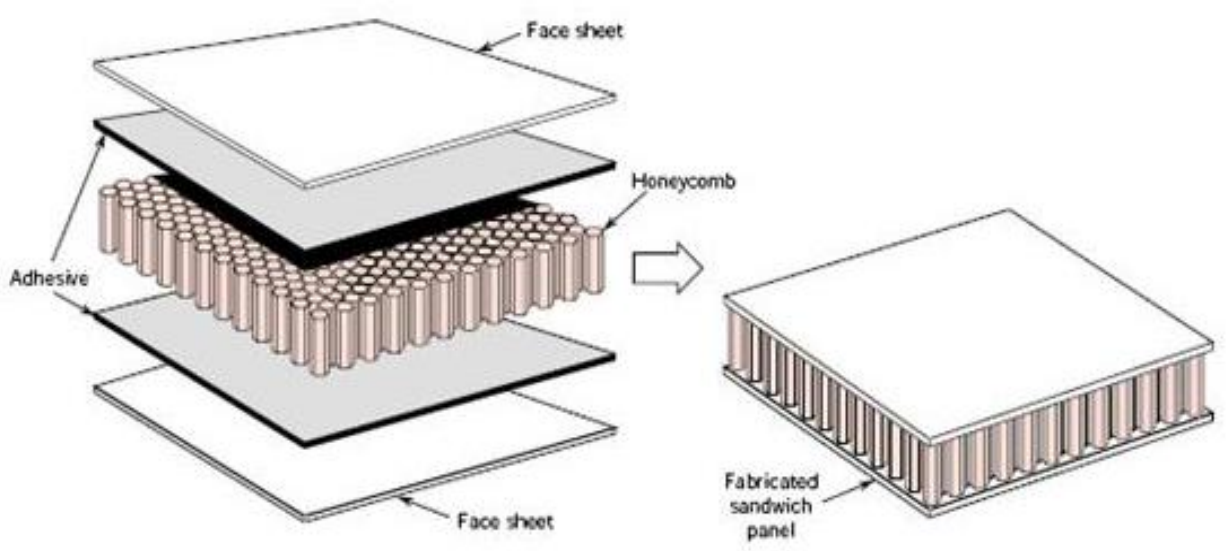

Figure 1.3 Schematic of honeycomb composite sandwich panel. [6]

\subsection{Manufacturing Techniques}

The processes of manufacturing composites vary widely, where each process has an advantage over the other and is used for a specific purpose. These processes include but are not limited to: hand lay-up, chopper head for spray-up, spray-up fiber and resin, vacuum bag molding, compression molding, vacuum resin infusion, filament winding, centrifugal casting, and pultrusion.

The hand lay-up method is quite common and advantages to this method are that it is can be cured at room temperature, it requires little technology, material cost is low, tooling costs are minimized, and the manufacturing of a large part is possible. However, disadvantages do exist for this manufacturing method which include: a poor working environment, laminate quality is dependent on the operator's skill, and thermosets pre-preg must be stored at sub-ambient temperatures to prevent from curing. The use of a chopper head for spray up of fiber and resin can be very advantageous due to its low cost tooling and established industry. Disadvantages to this method are that only short fibers can be used, waste is high, it produces resin rich laminates, 
and low viscosity resins are required. Vacuum bag molding of composites is another common manufacturing process because it can be used in combination with other machines to produce the highest quality laminate. Vacuum bag molding can cure solely with a nylon bag and vacuum mechanism at room temperature or can be cured under high pressures and temperatures in an autoclave to produce a high quality laminate. This method utilizes a wet lay-up or special pre-preg material prior to the vacuum process, where the lay-up of the composite usually consists of an epoxy that requires a long cure time. With the use of an autoclave, high quality composites and large sizes are possible. The low costs and clean manufacturing environments are a few advantages of the vacuum bag molding method. However, the capital cost of purchasing an autoclave and the cost of consumables (such as water) is very high and thus this method can be costly. Commercial aircraft wings are commonly manufactured in an autoclave with the use of vacuum bag molding, such as the Boeing 787 wings, where Boeing owns one of the largest autoclaves produced thus far [7].

Polymer composites require different types of manufacturing. Molding is the most common method for forming plastic polymers. The several molding techniques used include compression, transfer, blow, injection, and extrusion molding. For each, a finely pelletized or granulized plastic is forced, at an elevated temperature and pressure, to flow into, fill, and assume the shape of a mold cavity. Compression molding uses the appropriate amount of thoroughly mixed polymer and necessary additives are placed between male and female mold members as shown in fig. 1.4. When the mold is closed, heat and pressure are applied, causing the plastic to become viscous and flow to conform to the mold shape. Raw materials may be mixed and cold pressed into a disc before molding, which is called a preform. By preheating the preform, the molding time and pressure are reduced. This molding technique is used to fabricate both thermoplastic and thermosetting polymers; however, its use with thermoplastics is more time consuming and expensive than molding techniques such as extrusion or injection molding. In resin transfer molding, a variation of compression molding, solid ingredients are first melted in a heated transfer chamber. As the molten material is injected into the mold chamber, the pressure is distributed more uniformly over all surfaces. This process is used with thermosetting polymers 
and for pieces having complex geometries, one of its many advantages. Some other advantages to this method are that high fiber content is possible, styrene emission is reduced, and there is potential for automation. The disadvantages are a high tooling cost and size limitations, and the prediction of flow is difficult. Some of the many common applications of resin transfer molding are auto body panels, truck air deflectors, and wind blades [7].

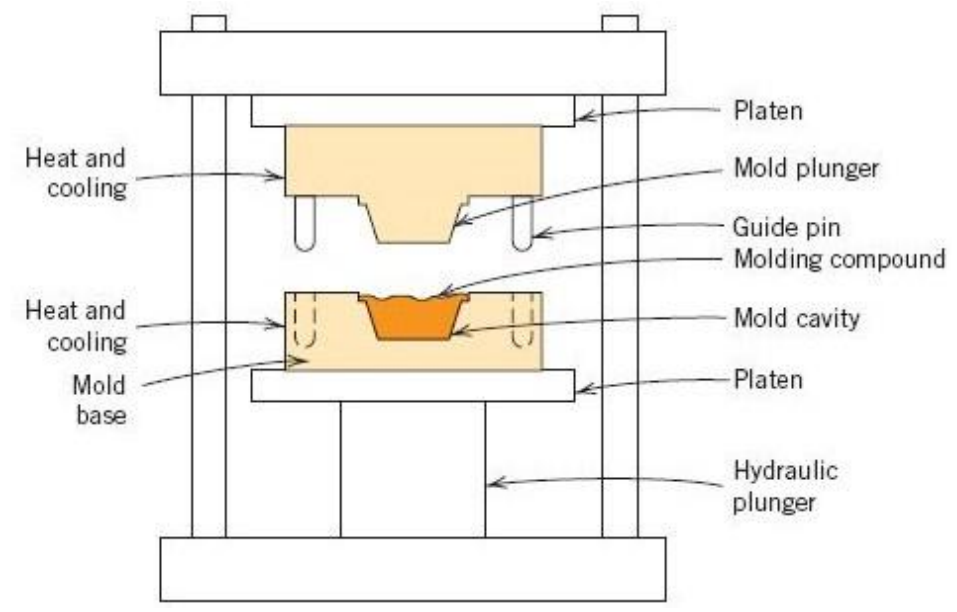

Figure 1.4 Compression Molding [7].

Further, there are many other fiber-reinforced composite manufacturing methods, such as vacuum resin infusion. This method is advantageous over transfer molding in that it has a much lower tooling cost, has no size restrictions, and can adapt to existing hand lay-up tooling. However, it has the disadvantages over resin transfer molding in that it can only have one molded surface, the injection time is limited by one bar of pressure, and there is less control over part thickness. This process also uses a contact mold, but preform is encapsulated by a sealed polymeric bag or lightweight laminate. Resin is then drawn through reinforcement under a vacuum. Marine industries, such as boat or ship companies, commonly use this method to produce products. Filament winding is a process by which continuous reinforcing fibers are accurately positioned in a predetermined pattern to form a hollow shape. This process utilizes a rotating mandrel and continuous fiber that feeds through nip rollers. Thereafter, it flows through a resin bath and flows through a moving carriage to creel the fibers. After the appropriate number of layers have been applied, curing is carried out either in an oven or at room temperature after which the mandrel is removed. Advantages of this process are its rapid process, high strength-to- 
weight ratios, various winding patterns as seen in fig. 1.5 (i.e. circumferential, helical, and polar), automation, and controlled resin content. Disadvantages are its shape limitations, unmolded outer surface, high equipment costs, and difficulty placing axial reinforcement. Common filamentwound structures include rocket motor casings, storage tanks and pipes, and pressure vessels $[7]$.
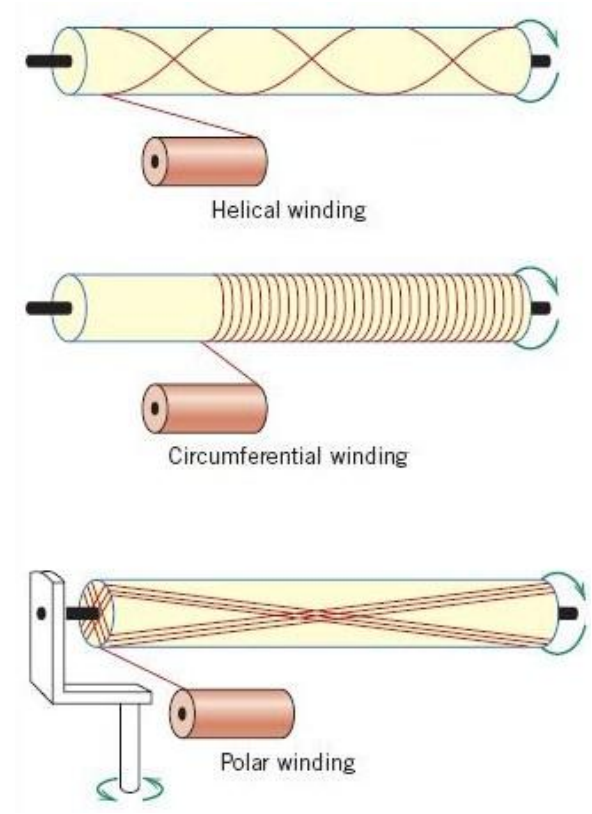

Figure 1.5. Winding techniques [7].

Pultrusion is a technique used for the manufacture of components having continuous lengths and a constant cross sectional shape. With this technique, continuous fiber rovings are first impregnated with a thermosetting resin and these are pulled through a steel die that conforms to the desired shape and established a resin-fiber ratio. The stock then passes through a curing die that is precision machined so as to impart the final shape; this die is also heated to initiate curing of the resin matrix. A pulling device draws the stock through the dies and also determines the production speed. Tubes and hollow sections are made possible by using center mandrels or inserted hollow cores. Pultrusion is a continuous process that is easily automated; production rates are relatively high, making it very cost effective. Furthermore, a wide variety of shapes are possible, and there is really no practical limit to the length of stock that may be manufactured [7]. Centrifugal casting is a method use to deposit reinforcement and matrix inside 
a cylindrical mold, where consolidation is done by mold rotation. Typical parts made by this process are pipes, boilers, pressure vessels, and cylinder liners for piston engines.

\subsection{Impact on Composite Structures}

A constant concern in composite structures is the effect of foreign object impacts because of the significant damage to the structure that often is undetectable by visual inspection. Impacts can vary from a common low velocity impact, for example, a tool dropped on a product, to the high velocity impact of debris on a commercial aircraft. Further, the most dangerous and least common type of impact is a high-velocity high-mass impact. An example of this type of impact is the impact of a bird-strike on an aircraft, as shown below in fig. 1.6. These collisions can not only damage the structure, but often lead to catastrophic events such as in-flight emergencies, though low-velocity impacts can be more dangerous due to their lack of detection. Overall, low energy impacts reduce the strength of composites and often do considerable subsurface damage. The most common damages seen in aircraft are due to impact of hail, debris upon take-off and landing, and tools dropped during maintenance. These types of events can typically be simulated with the use of a drop tower or air cannon.

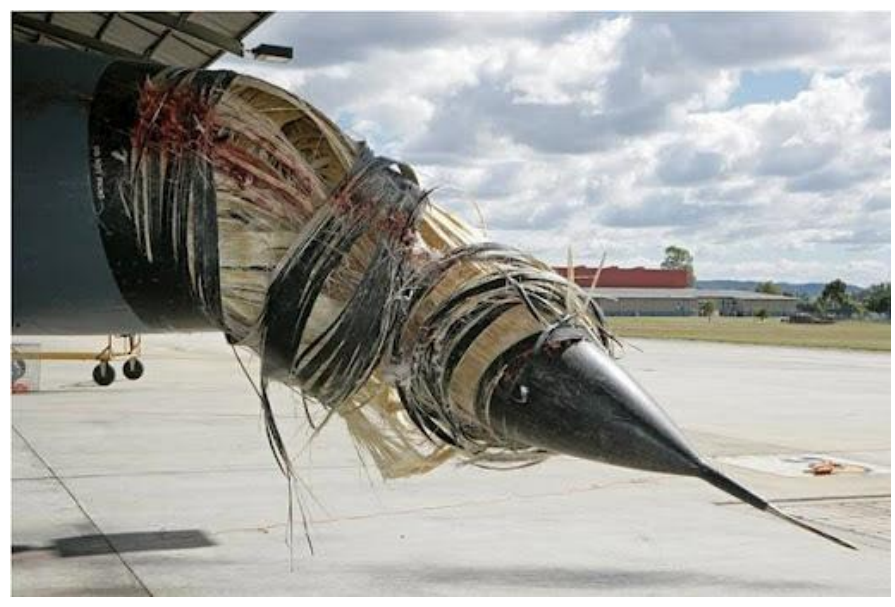

Figure 1.6. F-111 nose cone after bird-strike. [8]

In recent years, designers have discovered that designing an advanced high performance composite material is more than just achieving a high modulus and a material with the highest strength [9]. One of the most critical performance criteria is the material's ability to absorb energy and resist impact loadings. Unlike homogeneous isotropic materials, where the 
energy absorption and toughness can be determined through the use of fracture mechanics, the behavior of composites cannot be predicted. Fracturing processes for composites are much different from homogeneous materials as are energy absorptions predictions. Therefore, techniques such as drop weight testers or air-cannons are necessary for understanding the behavior of impact on composites. With a general understanding of impact behavior, the design of important structures such as aircraft, ships, and spacecraft, can be improved significantly. To improve the structural integrity of a structure that has been impacted, DADs may be utilized. The purpose of adding DADs to a structure is to retain the ultimate strength of the structure under static loading and maintain the fatigue life of the structure under fatigue loading. Therefore, in this research two DAD configurations were added to previously impacted composite sandwich beams. Static 4-point bend and 4-point bend fatigue testing was performed to observe any improvements to the structure compared to the impacted structures with no added DADs.

\subsection{Fatigue Behavior of Composites}

In general, fatigue life of classical engineering materials is challenging to predict, and for composite materials even more difficult. A composite laminate or lamina has various failure modes, and therefore each will respond differently to fatigue loading. Due to anisotropy in composites, the stress distribution can be several orders of magnitude different in any given direction while the strains may be the same. Consequently, general stress measurements such as Tresca or Von Mises used for metals cannot be used to predict the fatigue life of a composite material. The fatigue mechanisms in composites are similar to those seen in metals as they both involve a fatigue crack initiation and crack propagation phase. However, the fatigue crack initiation and crack propagation phases in composites are very dissimilar due to their complicated micro-mechanical process [10].

Typically in metals, a stress-life (S-N) diagram is plotted to represent the material's fatigue life. In composites such as lamina, it is more appropriate to plot strain versus the number of cycles. This is because there are some instances where the material constituents may be the same, but each has its own fiber volume fractions. An example of this is two unidirectional laminae of the same material constituents, where one has a high volume fraction (higher 
strength) than the other, but the strain to failure is governed by fiber properties and will usually be the same [10]. Therefore, the fatigue life should be the same in terms of strain, instead of the usual stress. In fatigue, where the load is applied parallel to the fibers, material fracture is more statistically significant due to the fact that fracture stress does not always occur at the same stress between two identical test specimens. Composite laminates are no different because they consist of a large number of fibers, where each fiber has a slightly different strength. At load levels near the static strength of the laminate, fibers fail at different load cycles, provided the stress is beyond the strength of the weakest fiber in the laminate [10]. During each cycle, the stress state is different due to the previously failed fibers. As the number of fiber failures becomes large enough in a given local area, the load redistribution in the area causes more fiber fractures to occur due to the stress concentration created. Thereafter, a crack forms and grows very rapidly during the next few load cycles, which leads to final failure of the specimen. Therefore, fatigue life of a composite can vary between any number of cycles because the process of fiber fracture is chaotic in nature, and does not involve any growth mechanism until the very last few load cycles [10].

Strength is greatly reduced in a unidirectional composite loaded at an off-axis angle to the fiber. Typical failure modes include delamination between fibers or matrix shear, and the fatigue limit is much lower in the off-axis loading case compared to the parallel loading case. This is because there are no discontinuities in the material that a growing crack can intercept in the off-axis case. On the contrary, any matrix crack forming in the case of loading parallel to fibers could arrest when meeting a matrix-fiber interface. Angle-plied laminates consist of multiple layers of off-axis layers and therefore the fracture mechanism is similar to that seen in off-axis laminates, where fiber matrix delamination is the main mode of failure [11]. However, in angleplied laminates, failure expands to delamination between layers, which is caused by inter-laminar stress present between layers. In the case of angle-plied laminate, the specimen does not fail completely upon a fiber-matrix crack in one layer which has grown the width of the specimen. This difference comes from the fact that there is another layer in another direction that will cross over the crack and carry the load, and therefore one crack does not have the ability to break the 
laminate. For a complete failure of the laminate, delamination between multiple layers must occur. However, an angle-ply laminate still precedes the fatigue limit of a unidirectional off-axis laminate due to the failure requirement of delamination in angle-ply laminates. In cross-ply laminates, the first indication of failure occurs after the fiber-matrix interface debonds in the 90degree layers. Subsequently, the stress concentrations create delamination in a comparable way as in the angle-ply laminates, where the laminate is reduced to a unidirectional lamina, though the fatigue limit of a cross-ply laminate is governed by the fatigue limit for delamination growth. Other common types of laminates include 0,90 , and \pm 45 -degree layers, where the main loading direction is applied in the zero-degree layers. Normally, the main mode of failure is found in failure of the 90-degree plies and transverse fiber debonding - which ultimately leads to delamination and overloading of the zero-degree fibers. The development of damage in composite laminates under fatigue is as follows: matrix cracking in layers of an off-axis lay-up, crack propagation, separation between plies (delamination), and fracture initiation. This illustration can be seen in fig. 1.7 below.

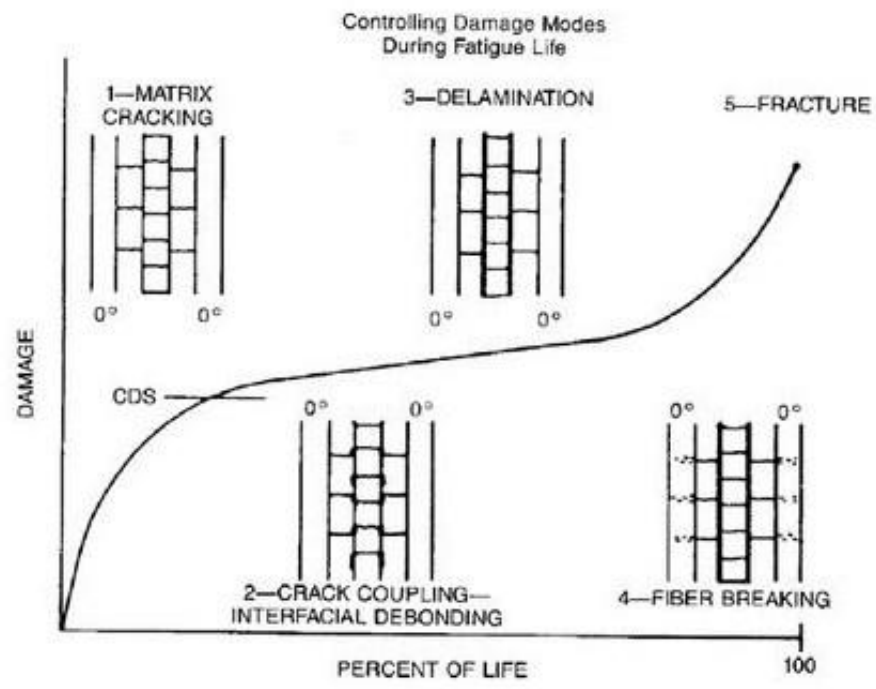

Figure 1.7. The development of damage in composite laminates. [10]

\subsection{Previous Research in Fatigue of Composite Materials}

Experimental research on fatigue in the area of composite materials has been widely developed since general theories do not exist. However, research efforts on fiber-reinforced composite sandwiches have not been as extensive. Soni et al. [12] studied the fatigue behavior of 
foam core carbon/epoxy composite sandwich beams at room temperature and low temperatures for their potential applicability in the hull materials of ships navigating in Polar Regions. In this study, four-point static flexural tests confirmed the effects of low temperatures on the strength, elastic limits, stiffness, and brittleness of the composite sandwich beams. Under fatigue loading conditions at low temperatures, the dominant failure mode was found to be core shear. Static finite element analysis was used to explain the effects of skin stiffness on the crack initiation location under fatigue loading conditions. Roosen et al. [13], investigated fatigue behavior of sandwich foam core materials, comparing the fatigue behavior among the different core materials. In this research effort, fatigue tests were conducted under a four-point bending test jig, where he concluded that PMI foams have good reliability in dynamically loaded demanding sandwich constructions. There has also been detailed research done on honeycomb aluminum cores. Harte et al. [14] examined the fatigue strength of sandwich beams with an aluminum alloy foam core under four-point bend. Upon completion of experimental and theoretical study, it was determined that a reduction in strength of sandwich beams exists for dynamic loading compared to static loading, where the set of possible collapse mechanics did not change. Further, it was found that sandwich beams are prone to shearing of the core under dynamic loading, similar to Roosen et al.

In addition to the investigation of fatigue on composite sandwiches, research has been expanded to composite sandwiches that have been previously impacted and/or delaminated. Gaedke et al. [15] researched the impact behavior and residual strength of sandwich structural elements under static and fatigue loading. In this investigation, it was found that the extent of impact damage in sandwich structures depends on the core material and the stacking sequence of the sandwich skin laminate. Further, the size, mass, and velocity of the impactor play a significant role in the ability of the component to absorb the shock at the impact point. Further, Gaedke discovered that delamination is easily detected around the impacted zone between skin and core of a foam core sandwich structure that has been previously impacted. In this case, the damage progress is triggered by the local buckling of the delaminated skin layer. A similar research investigation performed by Butler et al. [16], investigated the post buckling and fatigue 
limit of artificially delaminated composites. In this study, experimental studies were performed on plates with full-width and circular artificial delaminations at various depths. The experimental test results are compared with finite element data using Abaqus, which confirmed that two types of buckling modes can occur: thin-film buckling and overall buckling. For thin film specimens, it was found that propagation of the delamination does not occur within $10^{6}$ cycles when the maximum applied strain is below the strain that causes opening of delamination.

\subsection{Previous Research Efforts at Cal Poly}

This research is an extension of a project sponsored by the Department of the Navy, Office of Naval Research, under Award \# N00014-11-1-0359 which was awarded to the Aerospace Structures/Composites laboratory at Cal Poly in 2011. The project is titled "Damage Arrestment of Composite Sandwich Panels for Naval and Aircraft Applications" and has been researched extensively by several graduate students. This study is based off many of the previous theses under the awarded project that developed specific test methodology for manufacturing test specimens, where key aspects of each thesis is taken into account. Balabat et

al. [17] investigated the effects of damage arrestment device interaction with fasteners in a composite sandwich panel. Figure 1.8 depicts the various damage arrestment devices, made of fiberglass, that were used in Balabat's research. In this study, composite sandwich specimens were composed of Last-A-Foam FR 6710 polyurethane foam with two layers of LTM45 carbonfiber/epoxy weave, cured using a composite heat press. Damage arrestment devices were made of composite strips and the thickness of each damage arrestment device was varied and tested both under static loading and fatigue loading. Experimental static test results indicated that as the thickness of the device increased, the overall strength of the test structure also increased at a maximum thickness of 0.065 " and maximum strength increase of $109 \%$. Under fatigue loading, the test specimens with added damage arrestment devices showed similar fatigue trends as the control group, but overall the structural strength was increased with the addition. 


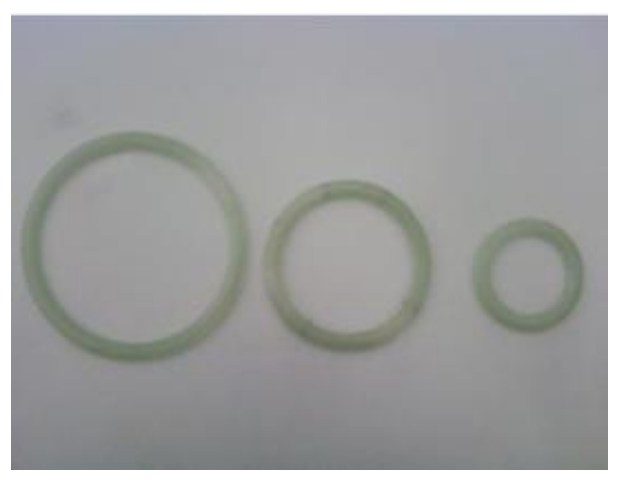

Figure 1.8. Circular DADs used in Richard Balabat's thesis [17].

Surano et al. [18] investigated the effectiveness of damage arrestment devices in delaying fastener-hole interaction failures in composite sandwich structures subjected to static and fatigue loading under increased temperatures. This study was performed concurrent with the study performed by Balabat and used the same type of test specimen seen in Balabat's research. The composite sandwich panels, with and without the addition of the damage arrestment devices, were subjected to static compression at a rate of $5 \mathrm{~mm} / \mathrm{sec}$ and compression-compression fatigue loading at $50 \%$ yield at an amplitude of $65 \%$, under temperatures $75,100,125,150,175$, and $200^{\circ} \mathrm{F}$. It was found that the most effective method to delay failure involved milling rectangular slots in the foam perpendicular to the holes and adding three additional layers of carbon fiber. With this method, the ultimate loads of the test specimens were increased by 97,87 , $100,131,96$, and $119 \%$ for each of the respective temperatures listed above. Fatigue loading showed the number of cycles remained the same with the addition of the damage arrestment devices, even with a $97 \%$ increase in yield loading. Overall, between the two studies performed by Balabat and Surano, the addition of damage arrestment devices to the polyurethane/carbon fiber sandwich panels increased the strength of the structure under static and dynamic loading.

Tran et al. [19] researched initially delaminated composite sandwich plates with damage arrestment devices under static and dynamic buckling. Composite test specimens differed from the previous two mentioned theses in that the face sheets were composed of fiberglass instead of carbon fiber, but included the same polyurethane foam core. Similar to the two previous theses mentioned, DADs were made of fiberglass. However, the strips were non-circular and 12-inches in length, as shown in fig. 1.9. Test plates were cured utilizing a vacuum infused resin (VRI) 
process. In this research, it was found that the introduction of the continuous damage arrestment key parallel to the in-plane loading provided a significant increase in the loading and buckling capabilities in comparison to the control test specimens without delamination and no embedded key. The continuous key placed parallel to the load vector increased the structural strength by $126 \%$ from a 1 -inch delaminated structure, with an $11 \%$ decrease from the non-delaminated structure. Further, failure modes of the experimental testing differed for the various configurations and delamination sizes. Failure modes included localized buckling of the face sheet, arrestment of the 1-inch delamination, and point load concentrated failure.

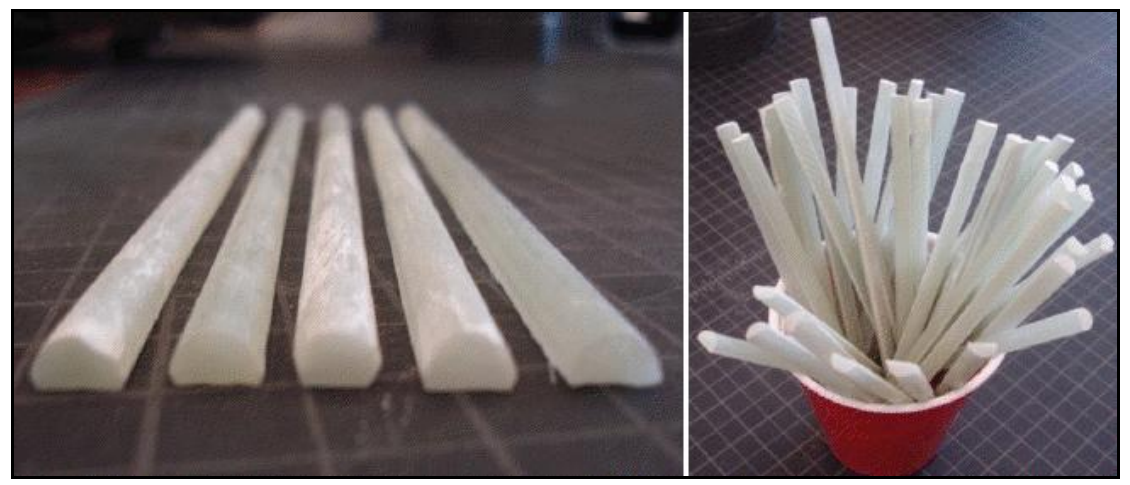

Figure 1.9. DADs used in Tony Tran's thesis [19].

Eswonia et al [20] examined the effect of shear keys and delamination in composite sandwich structures under static and fatigue loading. Sandwich structures were composed of fiberglass face sheets and a polyurethane core and cured using a vacuum resin infusion (VRI) process, similar to Tran's research. Experimental results showed that sandwich structures with an initial delamination and/or shear key in the foam core experienced a $70 \%$ reduction in ultimate strength when subjected to static loading. Additionally, sandwich structures with a shear key in the foam core had a fatigue life reduction of approximately $33 \%$. Orientation of the shear key was then changed from 0 to 90 degrees and initial tests showed a $30 \%$ increase in ultimate strength under static testing. A static numerical analysis was performed using Abaqus to verify the static behavior of the test specimens with no initial delamination and with 0.5 inch initial delamination.

Davis et al. [21] tested similar test specimens as seen in Balabat [17] and Surano [18], where the composite sandwich beam structure was composed of two layers of LTM45/CF1803 pre-impregnated carbon fiber and a Last-A-foam FR 6710 polyvinylchloride foam core. Test 
coupons were cured with the use of a composite press plate, similar to [17] and [18]. In this research, the mechanical characteristics were investigated under a static four-point bend. Damage arrestment devices, identical to Tran's, were introduced transversely and longitudinally in the beams to observe any increase in ultimate strength of the test specimens. A one-inch wide delamination was also introduced in the center of the beam to see if the damage arrestment devices improved the ultimate strength. Ten test samples were tested for each case and it was concluded that the addition of the damage arrestment keys in both configurations significantly increased the structural integrity of both the delaminated and non-delaminated control beams, at most by $66 \%$ for the non-delaminated longitudinal key case.

Lastly, Rider et al. [22] investigated the effectiveness of damage arrestment devices on the mechanical behavior of composite sandwich structures subjected to a low velocity impact. Test specimens were composed of four layers of LTM45EL/CF1803 bi-directional woven carbon fiber per face sheet and a General Plastics Last-A-Foam FR-6710 rigid polyurethane core, cured in an autoclave. Static four-point bend and vibration testing was performed on both impacted and non-impacted cases. Four different specimen cases were investigated in the 4-point bend test with and without being impacted: a control beam with no delamination or DAD, a control beam with a centrally located 1 -inch long initial delamination, a DAD key beam with two transverse DADs centrally located 1 -inch apart, and a DAD key beam with a centrally located initial delamination between two transverse DADs. Experimental results showed that the presence of DAD keys significantly improved the residual stiffness and ultimate strength of the damaged, under low velocity impact loading, including test cases with the presence of an initial face-core delamination. Further, vibration testing was performed on composite sandwich plates, with and without damage arrestment devices, with and without initial delamination, and with and without low velocity impact damage. Experimental results concluded that the damping characteristics significantly degraded for the control case after low velocity impact damage, but those with the damage arrestment devices retained more of the damping characteristics. Numerical analysis was performed to validate the experimental results using the LS-Dyna explicit and implicit FE solver. 


\subsection{Objective of Research}

The objective of this study was to determine the effects of the addition of damage arrestment devices to composite sandwich beams subjected to both static 4-point bend and 4point bend fatigue loading. Static 4-point bend testing was employed to determine whether DAD keys improved the flexural strength of composite sandwich beams with and without the presence of an initial face-core delamination. Four-point bend fatigue testing was employed to determine if the implementation of DAD keys in the composite sandwich beams increased the fatigue life of composite sandwich beams with and without the presence of an initial face-core delamination.

Composite sandwich beams were introduced to a low velocity drop weight impact event to see whether DAD keys reduced face sheet impact damage with the impact occurring directly on the DAD key. Further, an impact test was performed on composite sandwich beams to see whether DAD keys reduced the propagation of face-core delamination with the impact occurring directly between two DAD keys. Impact events were then introduced to initially delaminated composite sandwich beams to see whether DAD keys improved the damage and propagation of delamination. Fatigue tests were then performed on the impacted test specimens to see if DAD keys postponed face-core delamination propagation, and thus increase the fatigue life of the test

specimens. Ultimately, this research was performed to evaluate the effectiveness of DADs in previously impacted composite sandwich beams under fatigue loading

\subsection{Scope of Research}

The remainder of this research is detailed in chronological order, where the details of experimental, theoretical, and numerical methods and results are explained thoroughly upon final conclusion. Chapter 2 explains the methods of fabrication and testing procedures used to obtain the mechanical characteristics of each constituent material in the composite sandwich test specimens. Further, the method of manufacture used to create the completed test specimens used throughout the experiments is also explained. Chapter 3 discusses the experimental methodology and procedures performed for the static 4-point bend testing of the beams, impact of the test specimen beams, and fatigue testing of the beams. Chapter 4 presents the results of the experimentally investigated static 4-point bending of the test specimen beams, impact, and 
fatigue of the test specimen beams. Failure modes are examined and discussed in detail for the test specimens subjected to static 4-point bending, and impact loading and 4-point bend fatigue testing. Chapter 5 discusses the approach to modeling and analyzing the experimentally investigated tests using a numerical method. Further, chapter 5 discusses and compares the results of the experimentally and numerically investigated static 4-point bending of the test specimen beams. Lastly, chapter 6 discusses the conclusions derived from this research based on all of the experimental and numerical results. 


\section{Chapter 2}

\section{Specimen Manufacturing and Mechanical Characteristics}

This chapter discusses the manufacturing methods used to test constituent test coupons and the mechanical characteristics obtained thereafter for each material used in the composite sandwich beam. The test process for obtaining the mechanical characteristics is also detailed further. Important parameters obtained include the elastic modulus, Poisson's ratio, and density of the carbon fiber face sheets, polyurethane foam core, and fiberglass DAD keys. Lastly, the fabrication method of each composite sandwich beam case is discussed in detail.

\subsection{Carbon Fiber Face Sheets}

The face sheet material used in the composite sandwich beam test specimens was Advanced Composites Group pre-impregnated LTM45EL matrix with CF1803 bidirectional woven carbon fiber and can be seen in fig.2.1. There are several methods to cure pre-impregnated carbon fiber, but the chosen curing method was an American Autoclave Company autoclave which is located in the Cal Poly Aerospace Structures/Composites lab. This curing method was ultimately chosen because of its ability to produce high quality material with minimal manufacturing defects and inconsistencies. This method is used very commonly in industry because of its pressurized and closed chamber environment, which produces quality parts that are consistent. The curing method, obtained from the Advanced Composites Group specification sheet [30], consisted of a 16 hour dwell period at a temperature of $140^{\circ} \mathrm{F}$ and a pressure of 70 psi. This curing cycle is illustrated below in fig. 2.2. 


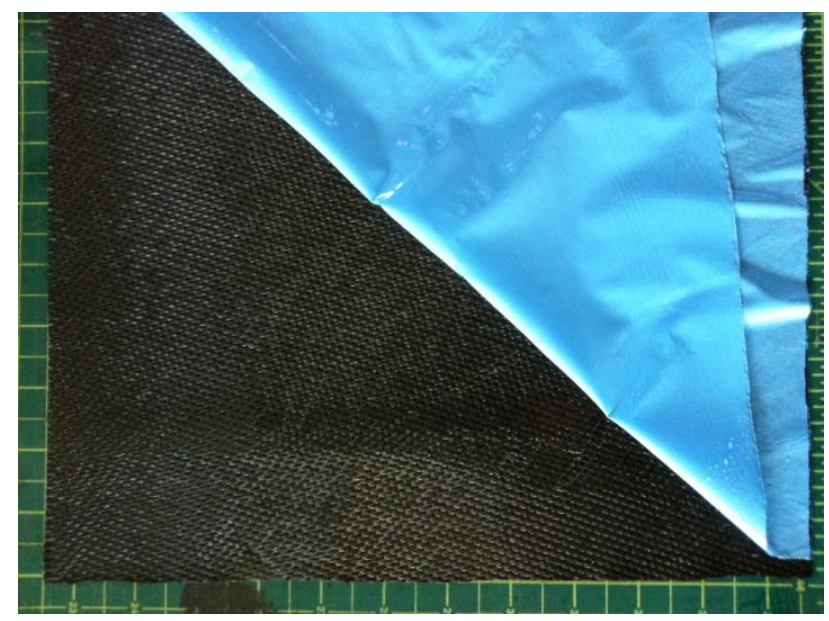

Figure 2.1. LTM45EL / CF1803 bidirectional woven carbon fiber

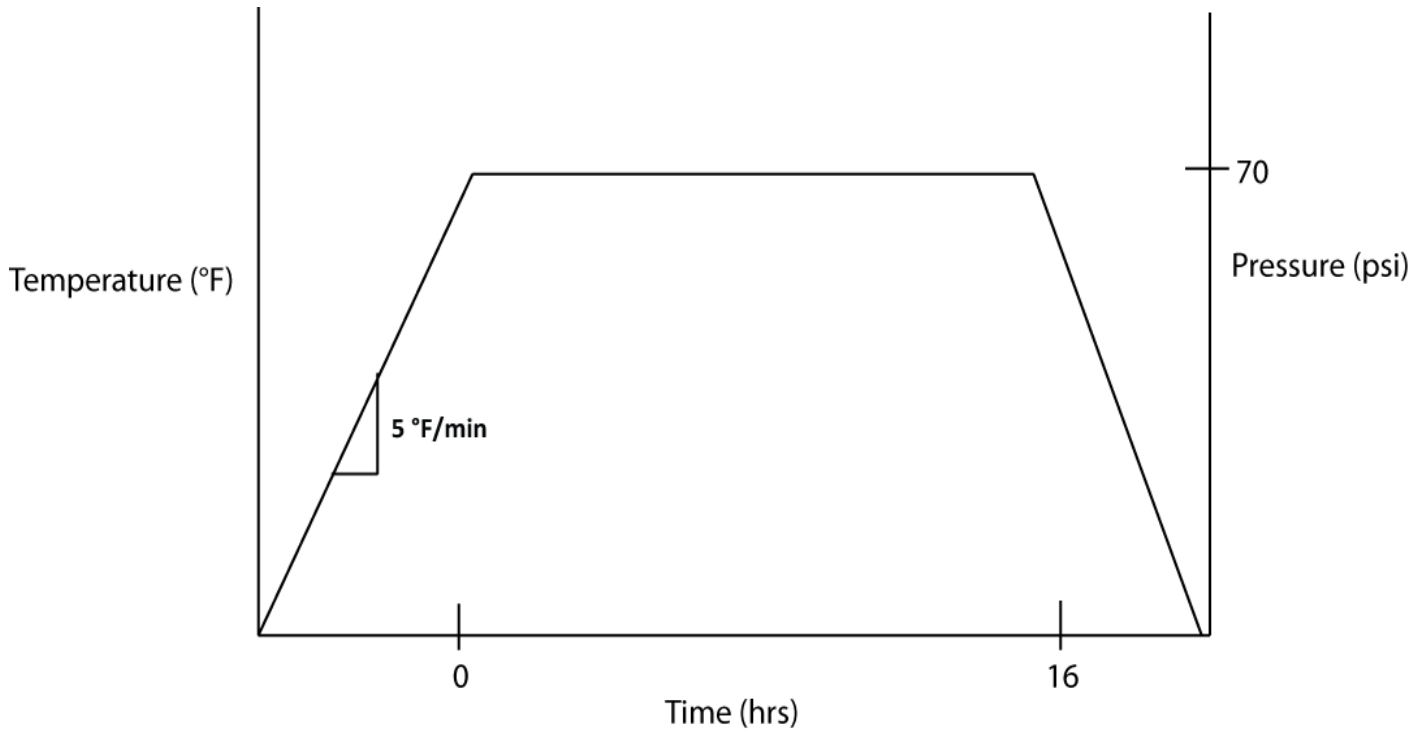

Figure 2.2. Autoclave cure cycle.

The face sheets used in the composite sandwich test specimens were each made up of two plies of Advanced Composites Group LTM45EL/CF1803 carbon fiber. Therefore, in order to obtain the correct mechanical characteristics, two-plies of 12-inch by 12 -inch pre-impregnated carbon fiber were used to manufacture a plate for testing. To prepare the specimen for curing, two 12 -inch by 12 -inch sheets of pre-impregnated LTM45EL/CF1803 carbon fiber were carefully cut using a utility knife. Two 14 -inch by 14 -inch sheets of porous release material were cut using scissors and used to reduce surface build-up of excess resin by placing both under and over the part. Breather material was implemented in the lay-up and placed over the part in order to allow 
for ideal airflow to the part and was cut to dimensions of 14 -inch by 14 -inch. A 14 -inch by 14 -inch sheet of non-porous material was used and placed directly under the part to allow for a smooth finish on the bottom surface. Lastly, a high temperature vacuum bag was cut to dimensions of 20inch by 20 -inch in order to contain the part. A vacuum-ported aluminum plate designed specifically for the autoclave was then prepared for the lay-up of the carbon fiber plate. To prepare the plate for easier part and excess resin removal post-cure, the aluminum plate was prepared with a high temperature mold release wax. After cutting each constituent material and prepping the aluminum plate, the lay-up process began. The lay-up sequence of materials to be placed on the aluminum plate was as follows: vacuum bag, breather material, porous material, two layers of bi-directional LTM45EL/CF1803 carbon fiber, porous material, and non-porous material. This stacking sequence can be seen in fig. 2.3 and 2.4, where General Sealants synthetic rubber vacuum bag sealant in tape form is used to create a tight seal between the high temperature vacuum bag and the aluminum plate.

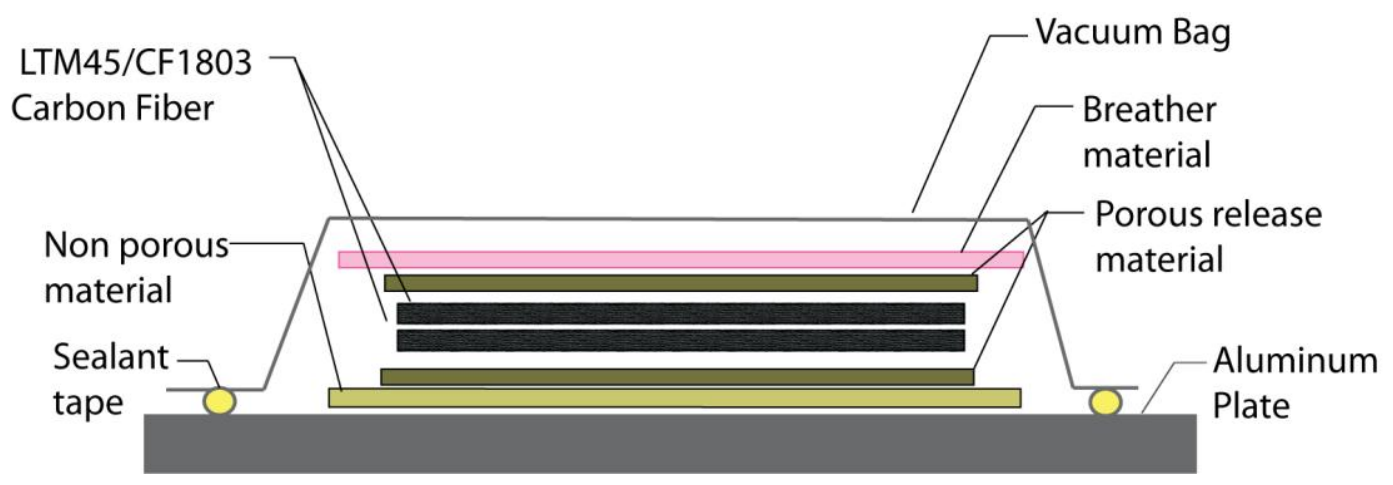

Figure 2.3. Schematic of layup and materials used to cure face sheets in the autoclave.

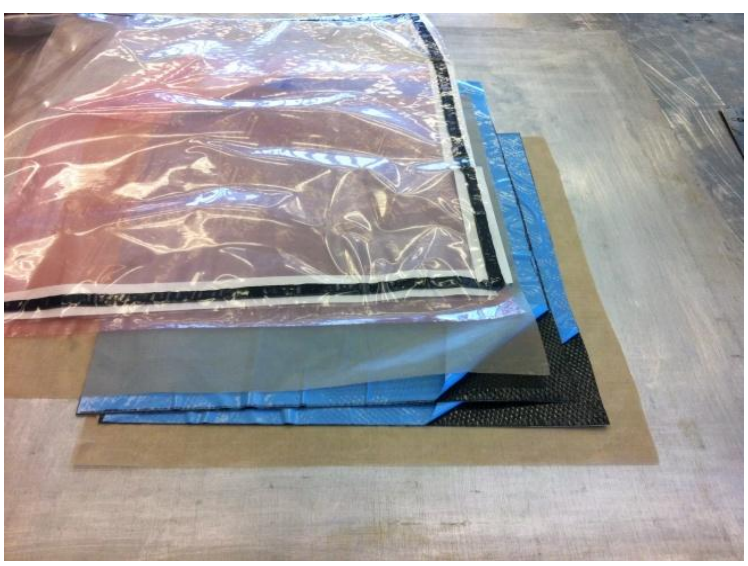

Figure 2.4. Lay-up Stacking Pattern of Materials for cure in the autoclave. 
After finishing the lay-up process and preparing the part for cure, the part was then placed inside the autoclave curing chamber and was put under an external vacuum for approximately 15 minutes by connecting the vacuum port on the aluminum plate to a pressure gage. This was employed to reduce inter-laminar voids and excess resin within the part, which is a result of atmospheric pressure applied to the part. Further, the amount of pressure applied to the vacuum dictated the quality of the seal of the vacuum bag. Bad vacuum seals were very detrimental to the bending stiffness and bonding between the core and face sheet, as they resulted in test plates with a fibrous finish due to the pre-impregnated resin burning out of the face sheet. After many trials and errors it was found that the pressure gage reading must be at least -10 in $\mathrm{Hg}$ in order to produce a part with optimum resin content. After applying an external vacuum pressure to the part, the part was then placed inside the autoclave and was prepared for curing, as shown in fig. 2.5. The autoclave used in this research was quite difficult to operate, thus detailed instructions to the operation of the autoclave can be found in Appendix A.

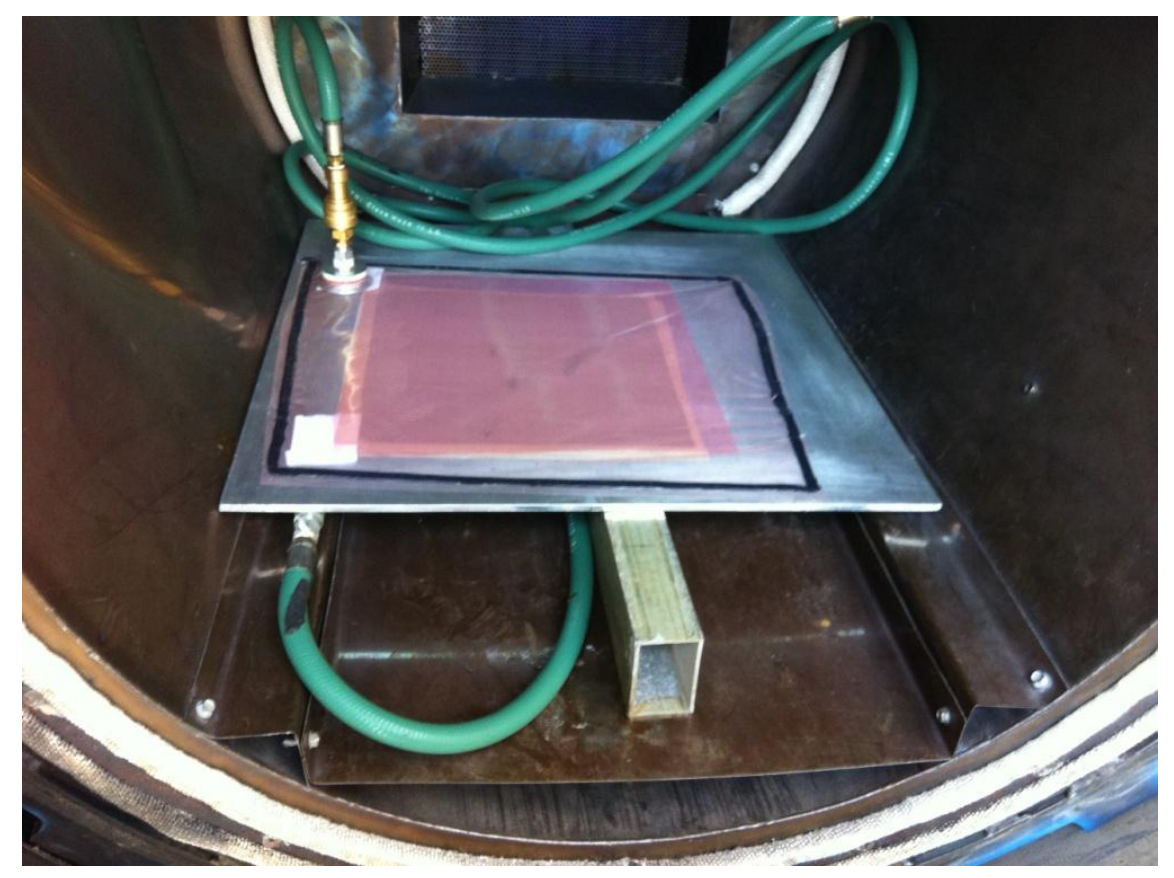

Figure 2.5. Carbon fiber face sheet lay-up placed inside autoclave cure chamber.

Following the successful completion of the sixteen hour cure cycle required for the test plate, the plate was prepared for test coupons required by the ATSM D3039 standard test"Standard Test Method for Tensile Properties of Polymer Matrix Composite Materials" [23]. Per 
the test standard, the plate was cut into 10 -inch long by 1 -inch wide test coupons with the use of a wet tile saw as shown in fig. 2.6 below. Test coupons were cut to tolerances specified by the standard, which required 1 percent for width variation and 4 percent for thickness variation. If any test coupons exceeded either tolerance value, the test coupons were not used for testing. Figure 2.7 shows the resulting cut test coupons, where ten of the most accurately cut test coupons were chosen for tensile testing.

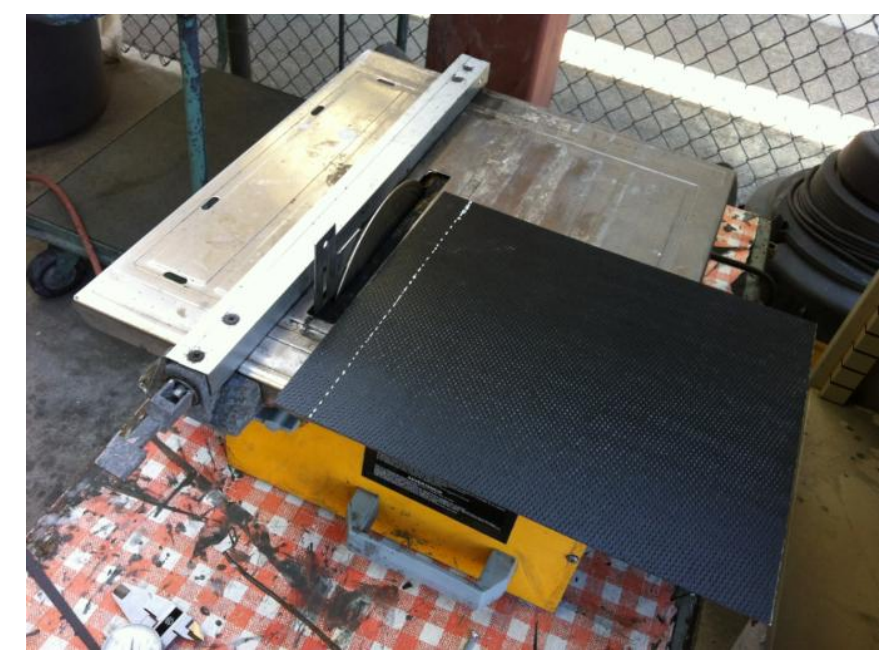

Figure 2.6. Wet tile saw used to cut test coupons for ASTM standard D3039.

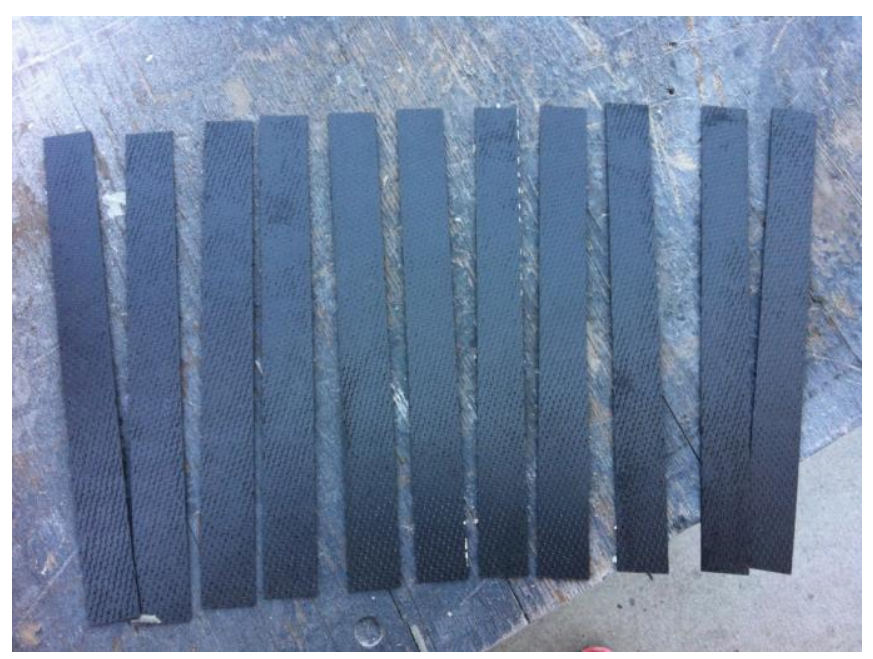

Figure 2.7. Test coupons cut to be tested according to ASTM standard D3039.

The tension test required the use of the Instron 8801 Servohydraulic Fatigue Testing System in the Cal Poly Aerospace Structures/Composites laboratory. This particular test fixture applies pressure to the coupon using wedge grips that provide a shear contacting force. To prepare the test coupons for tension testing, the standard calls for some type of grip material to 
be used in the wedge grips. Emery cloth was chosen as it provides a favorable grip between the carbon fiber test specimen and the Instron grips. In the past aluminum tabs have been used, but the cure time of the aluminum tabs to the carbon fiber test specimens and the inconsistency between each specimen ruled this method out. Each test specimen required a 1 -inch by 2 -inch cut emery cloth piece to be wrapped and taped around the ends, as shown in fig. 2.8. It must be noted that too much tape around the emery cloth could cause the grips on the Instron machine to lose hold of the test specimen; therefore, care was taken to ensure that only the required amount of tape was used.

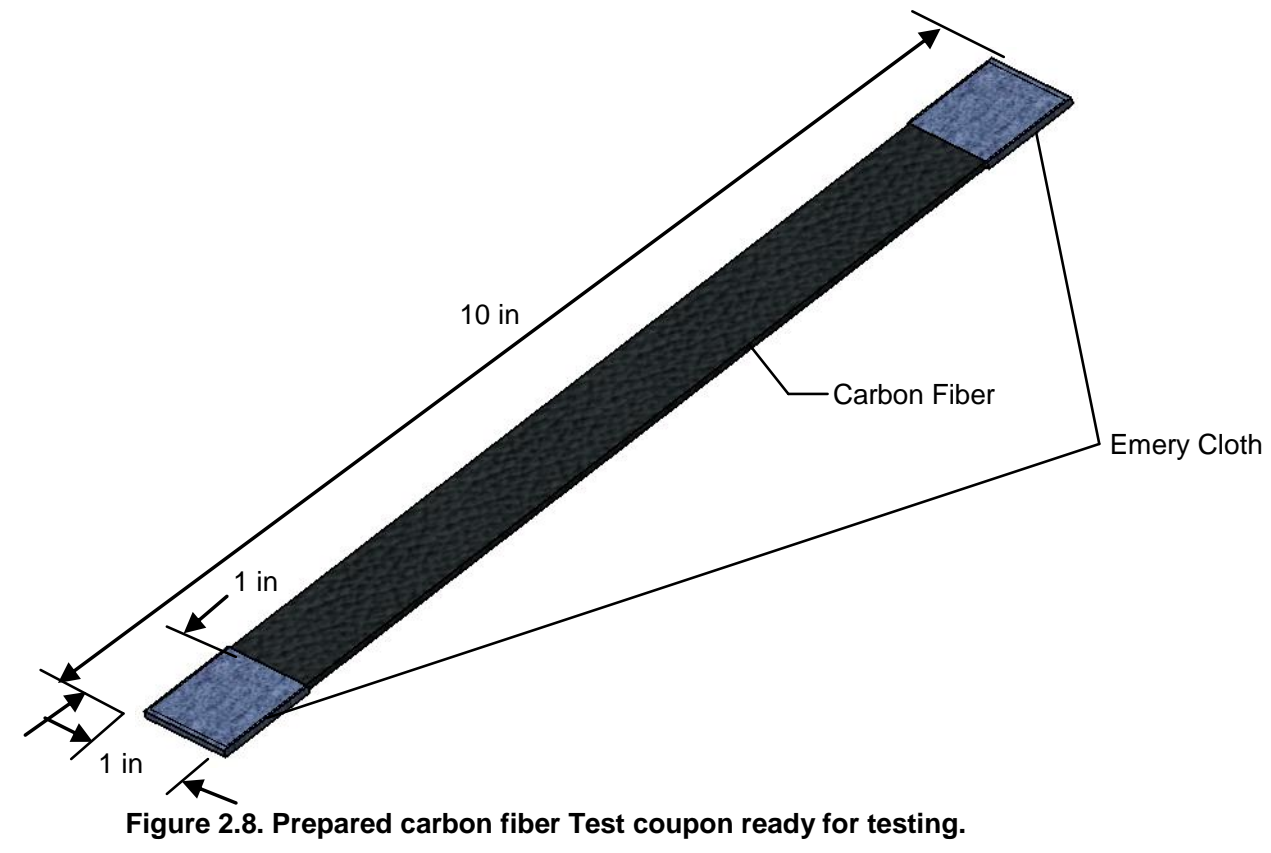

The standard tension testing method was then programmed into the Instron machine such that each sample was tested exactly the same way. Instron Bluehill software was used to program the Instron machine and determine important data parameters such as: stress, strain, load, elongation, and ultimate stress. Each test specimen was carefully placed into the Instron grips such that each specimen was parallel with the grips and perpendicular to the floor, as shown in fig. 2.9. The carbon fiber sample was loaded in tension using a constant crosshead speed of 0.05 -inches/minute until the applied load dropped by 40 percent per the standard. The data for each sample was recorded on the computer and then analyzed to obtain the Young's modulus. To obtain Young's modulus, the slope of the linear region of the resulting stress versus strain curve was found. 


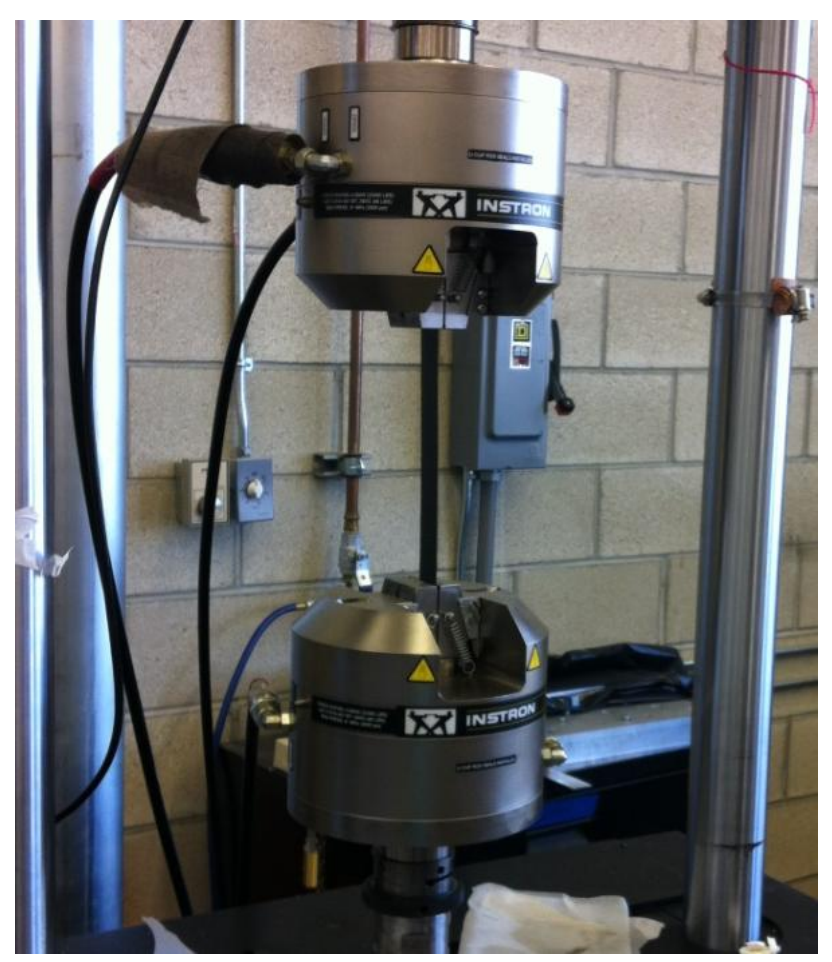

Figure 2.9. Carbon fiber test coupon secured in the Instron 8801 grips.

Figure 2.10 below shows a typical failure mode seen for the carbon fiber test coupon immediately after the tensile loading, which illustrates that the failure clearly initiates more than one inch from the wedge grip, a crucial requirement for a valid test according to the ASTM standard.

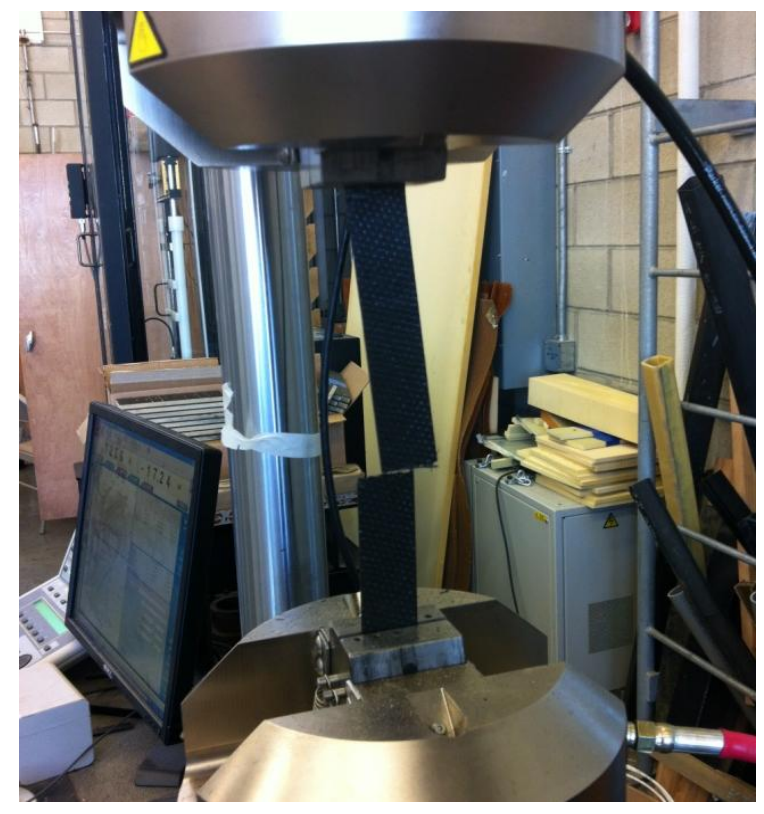

Figure 2.10. Typical failure mode of the carbon fiber test coupon. 
The Bluehill testing software yielded load versus extension curves for each of the test coupons, which resulted in ten curves. Stress versus strain curves were obtained thereafter, using the dimensions measured for each test coupon. Strain values were determined by dividing the measured extension of the test coupon throughout the duration of the test by the length of the test coupon, 10 inches. Stress values were obtained by dividing the measured load throughout the test by measured cross sectional area of each test coupon. The stress versus strain curves for each test coupon were plotted on a graph. The subsequent plot is shown in fig. 2.11 below. The ten curves are hard to distinguish because the slopes of the curves are so similar, which shows that the testing is very consistent. The average elastic modulus was found by determining the slope of the most linear region of each curve. The average ultimate stress was determined to be the maximum stress before failure of the test coupon. The resulting average elastic modulus values and average ultimate stress values are shown in Table 2.1. Ultimate stress is a very important parameter later when calculating the theoretical ultimate stress value of the entire sandwich beam structure. Table 2.1 gives a summary of the average elastic modulus of the ten test samples, the average ultimate stresses seen in the test specimens, and the standard deviations of each. Standard deviations were taken of the average elastic modulus and the average ultimate stress to make sure that the results were not misleading and that the curing method would be repeatable in the future. The average elastic modulus was determined to be $9.62 \times 10^{6}$ psi with a standard deviation of 4.48 percent and the average ultimate stress was determined to be 109,188 psi with a standard deviation of 8.51 percent. The standard deviations of the data are statistically acceptable and are considered repeatable and accurate. 


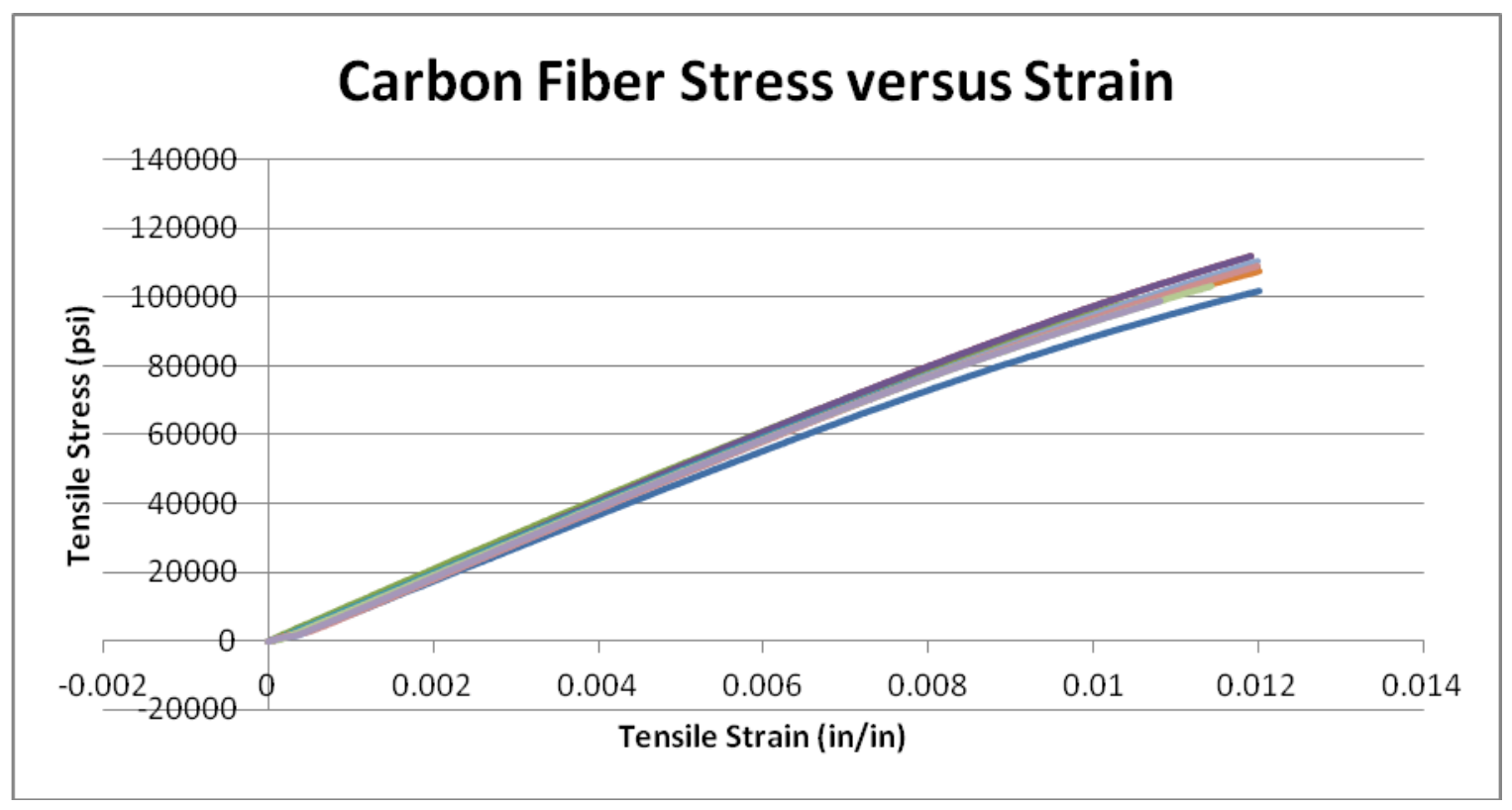

Figure 2.11. Tensile stress-strain curves obtained by experiment for face sheets.

Additionally, Poisson's ratio of the ACG LTM45EL/CF1803 carbon fiber face sheets was found experimentally following the ASTM E132 standard test- "Standard Test Method for Poisson's Ratio at Room Temperature" [24]. For this experimental test, the use of the Instron 8801 Servohydraulic Fatigue System in the Cal Poly Aerospace Structures/Composites laboratory was required, similar to the ASTM D3039 test standard. However, in addition to the typical tensile test where only the axial strain is found, the lateral strain must also be obtained.

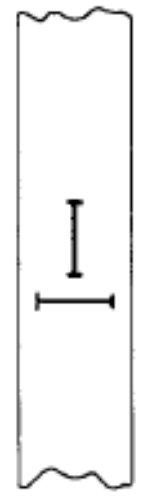

(a)

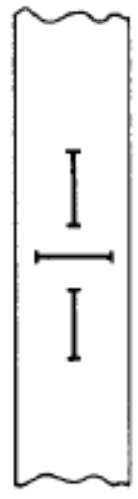

(b)

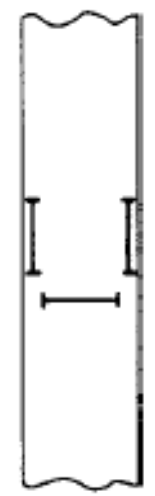

(c)

Figure 2.12. Possible strain gage locations on carbon fiber test coupons. 
Poisson's ratio was determined using strain gages attached to a P-3500 strain indicator. The strain indicator was connected to a National Instruments external connector box which was then connected to a data acquisition (DAQ) PC running Bluehill 2. The strain gages were placed both perpendicular and parallel to the length of the test coupon as shown under (a) in fig. 2.12, where the parallel strain gage obtained the axial strain and the perpendicular strain gage obtained the lateral strain. Copper leads were positioned on the test coupons and are then connected to the strain gage leads by two wires. Test coupons were cut to the same dimensions and prepared as seen in the test following ASTM D3039, where each coupon measured to be 10inches long by 1 -inch wide. The carbon fiber samples were loaded in tension using a constant crosshead speed of 0.05 -inches/minute, similar to ASTM D3039, but loaded to a maximum of $3000-\mathrm{lb}_{\mathrm{f}}$ to avoid failure. Eight test coupons were tested on the Instron 8801 testing machine in combination with Bluehill 2 software, attached controller, and data collection PC. Poisson's ratio was determined by dividing the slopes of lateral and axial strains by the applied load of a material which can be seen in equation 2.1 .

$$
v=\frac{\partial \varepsilon_{t} / \partial P}{\partial \varepsilon_{l} / \partial P}
$$

Axial and transverse strains were recorded by the strain gage external data channels. Data was output from Bluehill 2 into a raw data file. This was then opened in Microsoft Excel and the data was organized so the chart contained the axial and transverse strain gage data. Thereafter, average strain vs. applied load curves were plotted per the ASTM standard. As shown in fig. 2.13, there are two groups of very different sloped curves. The group with the shallower slope is defined as the transverse strain and the steeper slope is the axial strain. 


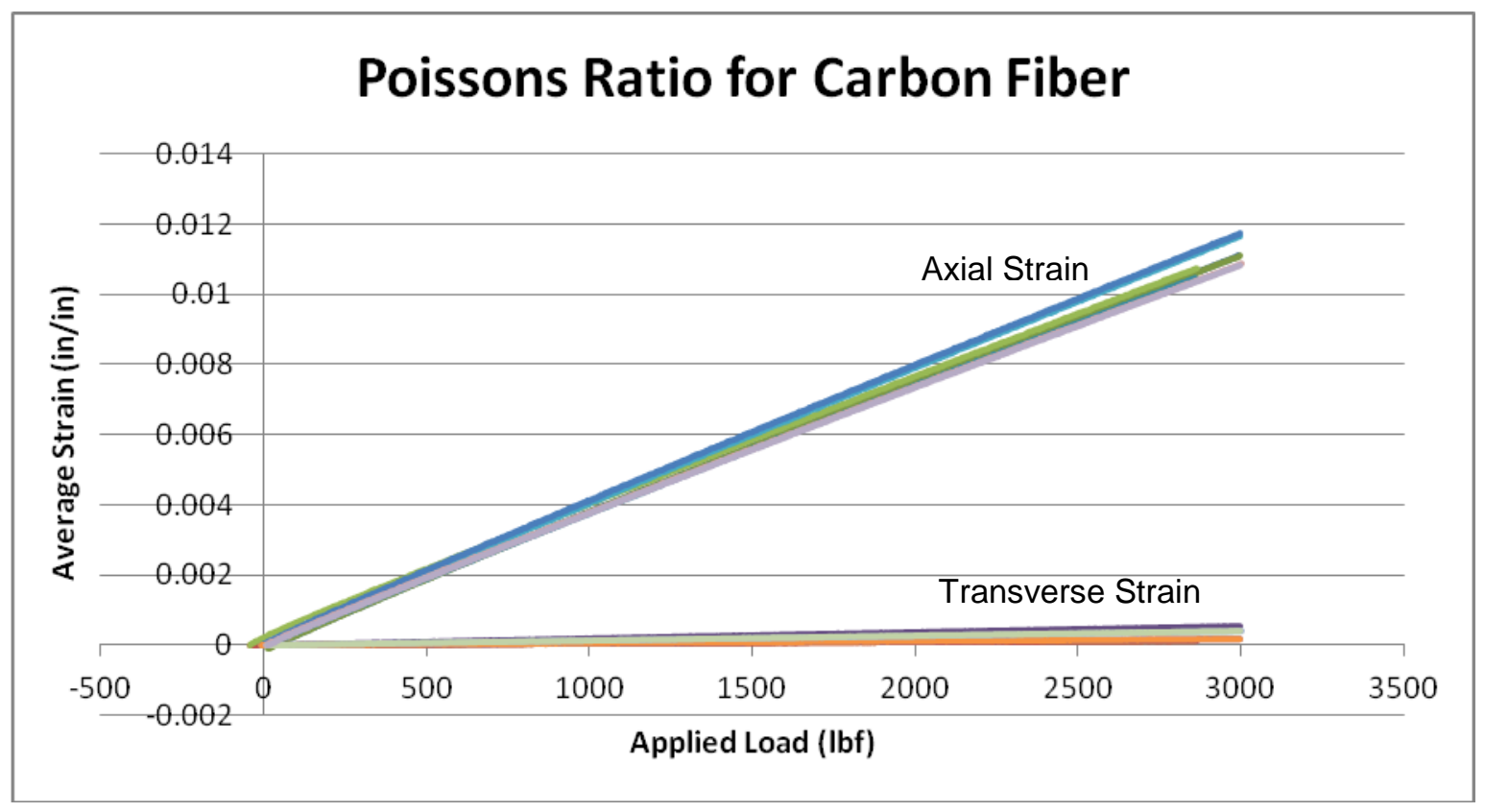

Figure 2.13. Plot of average strains versus applied load for determination of Poisson's ratio of Face sheets.

The measured Poisson's ratio was determined to be 0.096 with a standard deviation of 9.86\%. This Poisson's correlated closely to what was expected for a bidirectional weave which is often assumed to be between 0.09 and 0.1 [22].

Some important design parameters in composite materials include fiber volume fraction and laminate thickness. Volume fractions are used in design to calculate composite properties. Volume fractions almost always refer to fiber content. Weight fractions are used in manufacturing of composite materials. They may refer to fiber or resin in most composites. The thickness of a composite laminate depends on the amount of reinforcement and the relative amount of resin included in the composite laminate. In design perspectives, a laminate with a high fiber volume fraction will be thinner than one with a lower fiber volume fraction, since it will have less. Thus, we can say that the laminate thickness is inversely proportional to the fiber volume fraction. With the laminate thickness and volume ratio, one can design an optimal composite material for each specific need.

A composite material cannot contain $100 \%$ fiber. Therefore, in order to determine the amount of fiber in the composite, the volume fraction must be calculated. The volume fraction is used in design to calculate composite properties, which almost always refers to the fiber content. 
To obtain the volume fraction of the LTM45/CF1803 carbon fiber face sheets, a chemical matrix digestion was performed. To prepare for the chemical matrix digestion, the composite material was cut into squares that were 1 -inch by 2 -inches. The weight of the metal plate that each test sample was placed on was weighed on a digital scale to get the tare value as shown in fig. 2.14 below.

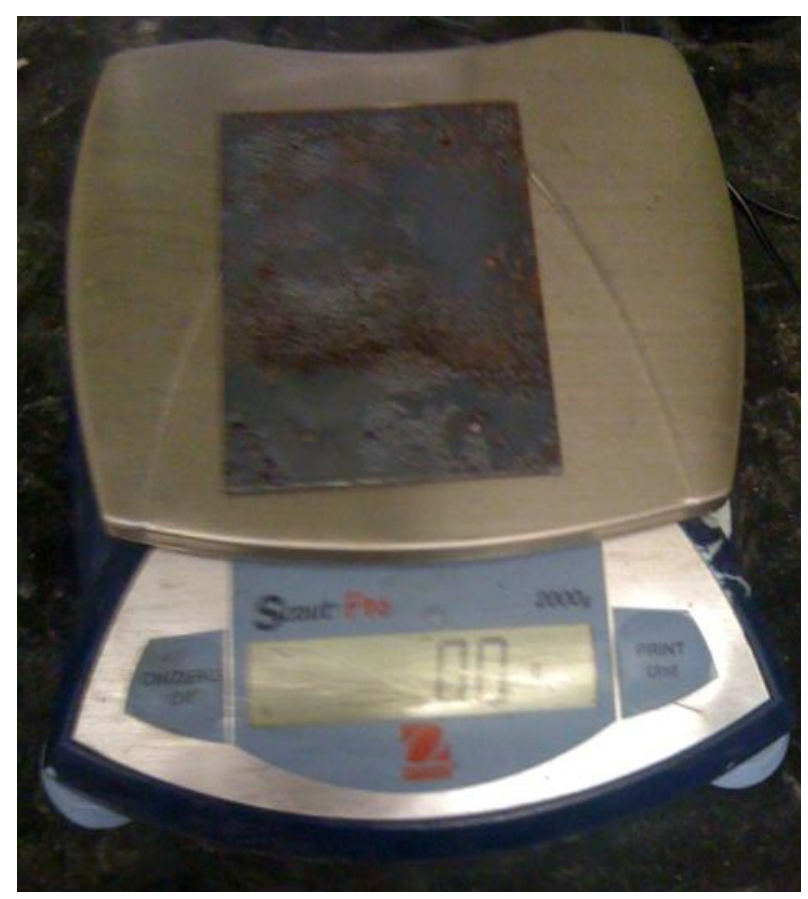

Figure 2.14. Plate used in experiment on a digital specimen weighing scale.

After recording the weight of the plate, the 1 -inch by 2 -inch composite samples were then weighed by placing each sample onto the plate and weighing both the plate and sample. This was repeated for ten test samples to ensure statistical accuracy. After weighing each composite sample and plate, the chemical matrix digestion was then ready to be performed. The chemical matrix digestion can be performed by either using chemicals or the resin burn-off method. For this experiment, the resin burn-off method was chosen.

To perform the resin burn-off method, the plate and sample were placed into an oven as shown in fig. 2.15, where each sample was heated for 45 minutes at approximately 1250 degrees Fahrenheit, in order to burn off the matrix in the composite. Once the matrix was completely burned off, the plate and composite sample were then reweighed, completing the chemical matrix digestion. Again, this was repeated for ten samples. 


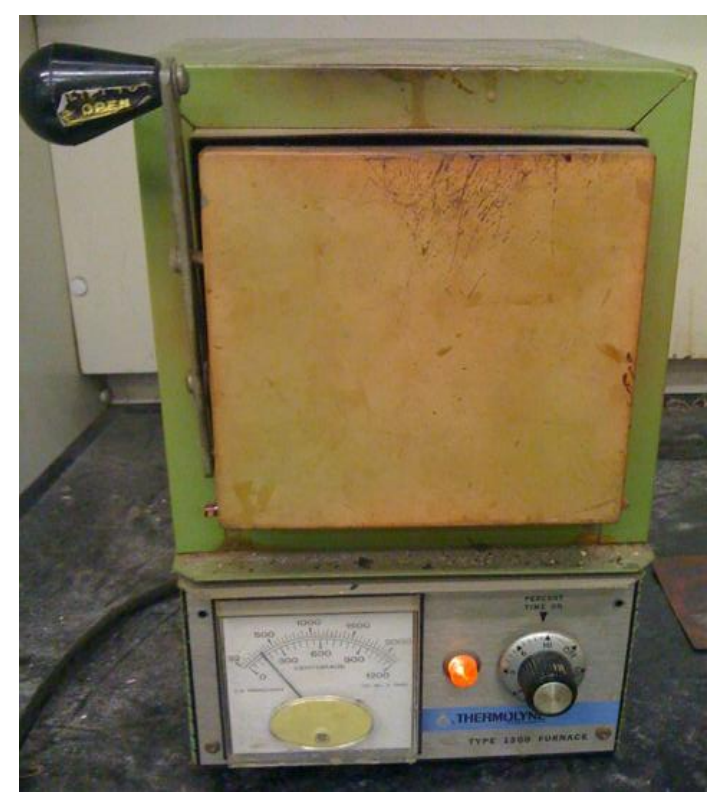

Figure 2.15. Oven used in experiment to complete resin burn-off procedure.

The density was then found by dividing the masses of the samples by the volumes of the samples, which came out to be an average of $94.84 \mathrm{lb} / \mathrm{ft}^{3}$. To validate the experimental density, the theoretical value was calculated using the densities of each constituent along with the volume fraction of the laminae. The density of the LTM45 resin and CF1803 carbon fiber was provided by the Advanced Composites Group Company's technical data sheet [30] and was 1.24 and 1.76 grams per cubic centimeter, respectively. With the volume fraction of 60 percent known, equation 2.2 can be used to find the density of the entire laminate:

$$
W_{\text {total }}=V_{\text {fibers }} * \rho_{\text {fibers }}+V_{\text {matrix }} * \rho_{\text {matrix }}
$$

With this equation, a theoretical value of the density was calculated to be $96.89 \mathrm{lb} / \mathrm{ft}^{3}$, which differs only by 2.1 percent when compared with the value found through experimentation. A summary of the mechanical characteristics of the carbon fiber face sheets is shown in Table 2.1. 
Table 2.1 Mechanical characteristics summary of LTM45EL/CF1803 carbon fiber

\begin{tabular}{|c|c|}
\hline \multicolumn{2}{|c|}{ CARBON FIBER MECHANICAL CHARACTERISTICS } \\
\hline Average Elastic Modulus (psi), ASTM D3039 & $9,619,014$ \\
\hline Elastic Modulus Standard Deviation (psi) & 431,484 \\
\hline Elastic Modulus Standard Deviation (\%) & 4.48 \\
\hline Average Ultimate Stress (psi), ASTM D3039 & 109,188 \\
\hline Ultimate Stress Standard Deviation (psi) & 8,513 \\
\hline Ultimate Stress Standard Deviation (\%) & 8.51 \\
\hline Average Density (Ib/ft ${ }^{3}$ ) & 94.84 \\
\hline Density Standard Deviation (lb/ft ${ }^{3}$ ) & 5.83 \\
\hline Density Standard Deviation (\%) & 6.15 \\
\hline Average Poisson's Ratio, ASTM E132 & 0.096 \\
\hline Poisson's Ratio Standard Deviation & 0.0095 \\
\hline Poisson's Ratio Standard Deviation (\%) & 9.86 \\
\hline
\end{tabular}

\subsection{Foam Core (GP Last-A-Foam FR-6710)}

Testing done for the General Plastics FR 6710 LastaFoam, used in the composite sandwich beam, followed the standard ASTM D1621 [25]. In this standard, the compressive properties of rigid cellular plastics can be found. However, in this standard, each test specimen must be at least two inches in height. Unfortunately, the PVC foam used in the composite sandwich beams analyzed in this research has a depth of only 0.5 -inches. This made this experiment difficult in that four 2 -inch by 2 -inch by 5 -inch squares were stacked and compressed according to the standard. This procedure produced data to plot a load-deformation curve, which yielded the compressive stress at any load and the effective Young's modulus. Since the sheets were stacked in order to obtain the correct test specimen dimensions, the samples compressed more than if there was just one homogeneous sample. This yielded significantly lower elastic moduli and ultimate strength values, compared with the given manufacturers values. A summary of the results comparing both the experimental values and manufacturer's values are found in Table 2.2. These results were obtained through an experiment performed by Richard Davis [21] in his thesis. 
Table 2.2 Mechanical properties of PVC foam [21]

\section{GP LAST-A-FOAM FR 6710 MECHANICAL PROPERTIES}

\begin{tabular}{|l|l|l|}
\hline & Manufacturer's Value & Experimental Value \\
\hline Elastic Modulus (psi), ASTM D1621 & 13,000 & 7,700 \\
\hline Elastic Modulus Standard Deviation(psi) & & 175.5 \\
\hline Elastic Modulus Standard Deviation (\%) & & 2.28 \\
\hline Ultimate Strength (psi), ASTM D1621 & 351 & 270 \\
\hline Ultimate Strength Standard Deviation(psi) & & 17.7 \\
\hline Ultimate Strength Standard Deviation (\%) & & 6.56 \\
\hline Poisson's Ratio, ASTM E132 & & \\
\hline Poisson's Ratio Standard Deviation & & \\
\hline Poisson's Ratio Standard Deviation (\%) & & 9.31 \\
\hline Density (Ib/ft $\left.{ }^{3}\right)$ & 10 & 0.16 \\
\hline Density Standard Deviation & & 1.62 \\
\hline Density Standard Deviation (\%) & & \\
\hline
\end{tabular}

\subsection{Damage Arrestment Device}

The mechanical characteristics of the damage arrestment devices used in this research were determined in an experiment performed by Kodi Rider in his master's thesis [22]. In this experiment, tensile tests were performed on unidirectional fiberglass test coupons. Test coupons were manufactured using a wet layup technique and composed of Jamestown Distributers FIB947 woven-roving fiberglass strands and a mixture of AEROPOXY PH3660 hardener and PR2023 epoxy resin. Tensile properties were found following ASTM D3039 test standard [26] and tests were performed on the Instron 8801 Servohydraulic Fatigue Testing System in the Cal Poly Aerospace Structures/Composites laboratory. Experimental tensile testing was performed with the fibers in the test coupons loaded parallel to loading and perpendicular to loading. A summary of the results found through this experiment can be found below in Table 2.3. 
Table 2.3 Mechanical properties of fiberglass damage arrestment device [22]

\begin{tabular}{|l|l|}
\hline \multicolumn{2}{|l|}{ DAMAGE ARRESTMENT DEVICE MECHANICAL PROPERTIES } \\
\hline Fibers Loaded Parallel to Loading & Experimental Value \\
\hline Elastic Modulus (psi), ASTM D3039 & $3.30 \times 10^{6}$ \\
\hline Elastic Modulus Standard Deviation (\%) & 17.2 \\
\hline Ultimate Strength (psi), ASTM D3039 & 11,985 \\
\hline Ultimate Strength Standard Deviation (\%) & 14.0 \\
\hline Fibers Loaded Perpendicular to Loading & \\
\hline Elastic Modulus (psi), ASTM D3039 & $6.24 \times 10^{5}$ \\
\hline Elastic Modulus Standard Deviation (\%) & 13.1 \\
\hline Ultimate Strength (psi), ASTM D3039 & 1,522 \\
\hline Ultimate Strength Standard Deviation (\%) & 14.0 \\
\hline
\end{tabular}

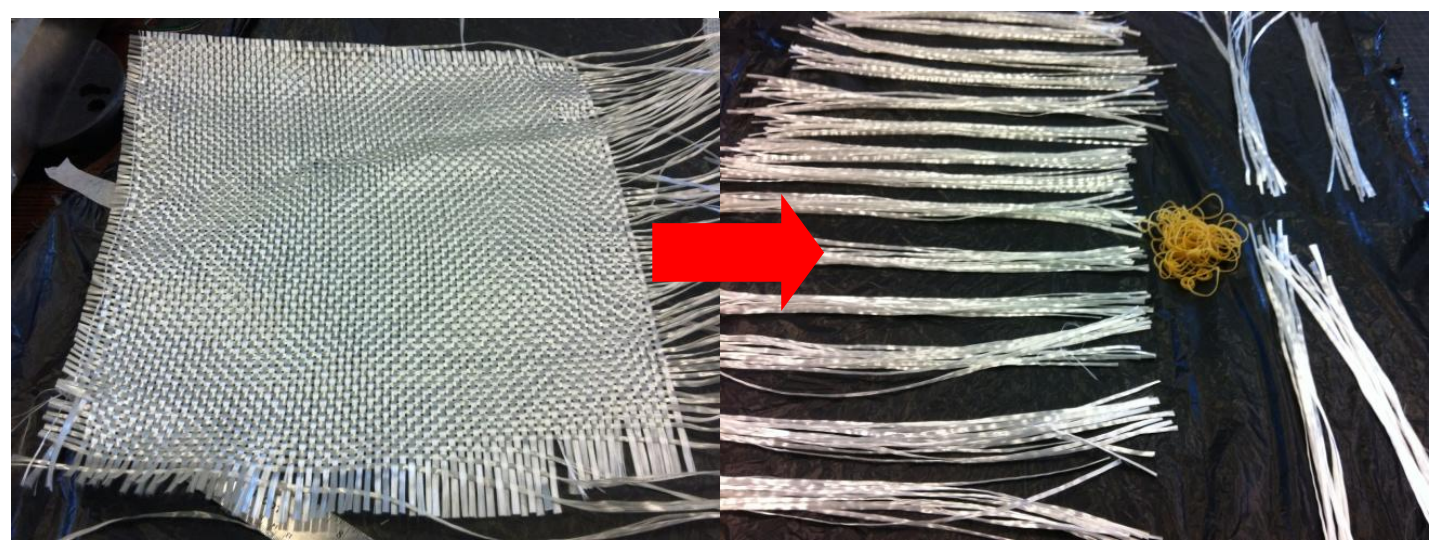

Figure 2.16. Woven roving fiberglass separated into unidirectional fiberglass strands.

The DADs were employed in this research in order to evaluate their potential to increase the ultimate strength in the delaminated beam case. The DADs were fabricated using Jamestown Distributers FIB-947 woven-roving fiberglass strands and by separating each strand as shown in fig. 2.16, where each strand was 14-inches in length. Each fiberglass bundle was cut to this length so that it may overlap the mold that the bundles were laid-up in. The mold, shown in fig. 2.17 , is made of aluminum and can hold up to 22 bundles of fiberglass. Bundles were composed 
of fifteen strands each and tied together using a rubber band. Each bundle created a 0.125 -inch radius semicircular DAD.

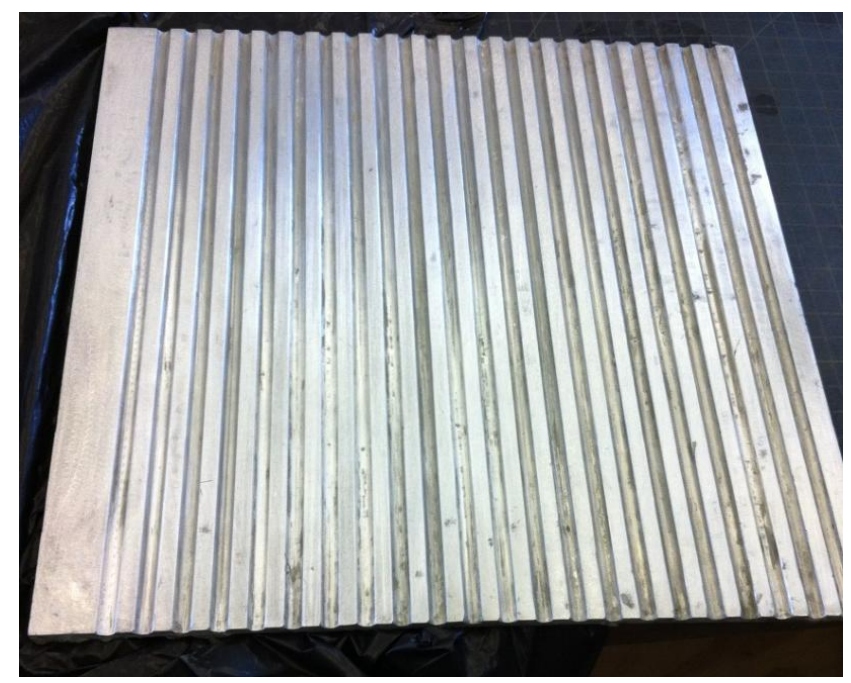

Figure 2.17. Aluminum mold for DADs.

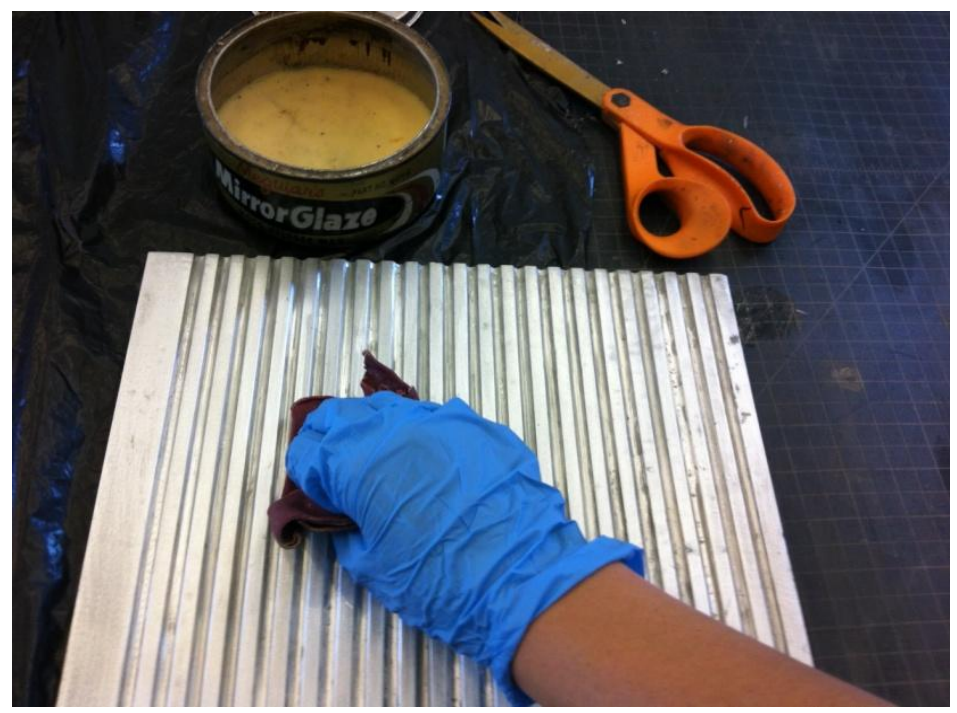

Figure 2.18. Mold release wax used to separate curved fiberglass from mold.

Before placing each fiberglass bundle in the mold, the aluminum mold was waxed with a releasing agent that allows for easy removal of the cured fiberglass DADs. This mold release wax was applied using a cloth until the entire mold was covered with the wax as depicted in fig. 2.18. 
Once the mold was prepared, the fiberglass bundles were ready for a wet layup, as shown in fig. 2.19.

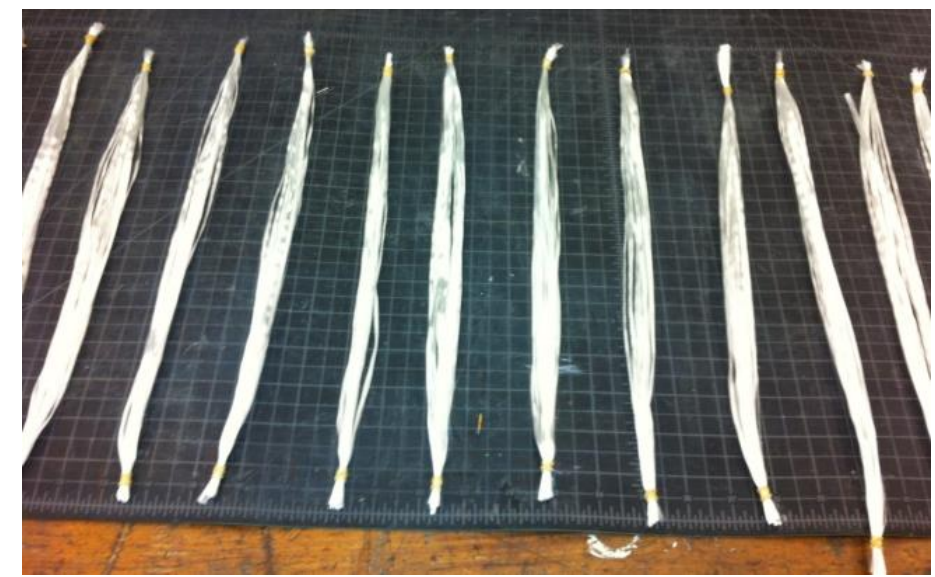

Figure 2.19. Bundles composed of 15 strands of unidirectional fiberglass.

To prepare for the wet layup, a mixture of AEROPOXY PR2032 Epoxy Resin and PH3660 Epoxy Hardener was prepared. The matrix material was composed of 100:27 resin to hardener and care was taken to ensure that the matrix was thoroughly mixed. Each bundle was then saturated thoroughly with the resin mixture, twisted at both ends, and placed inside each slot in the mold, as shown in fig. 2.20.

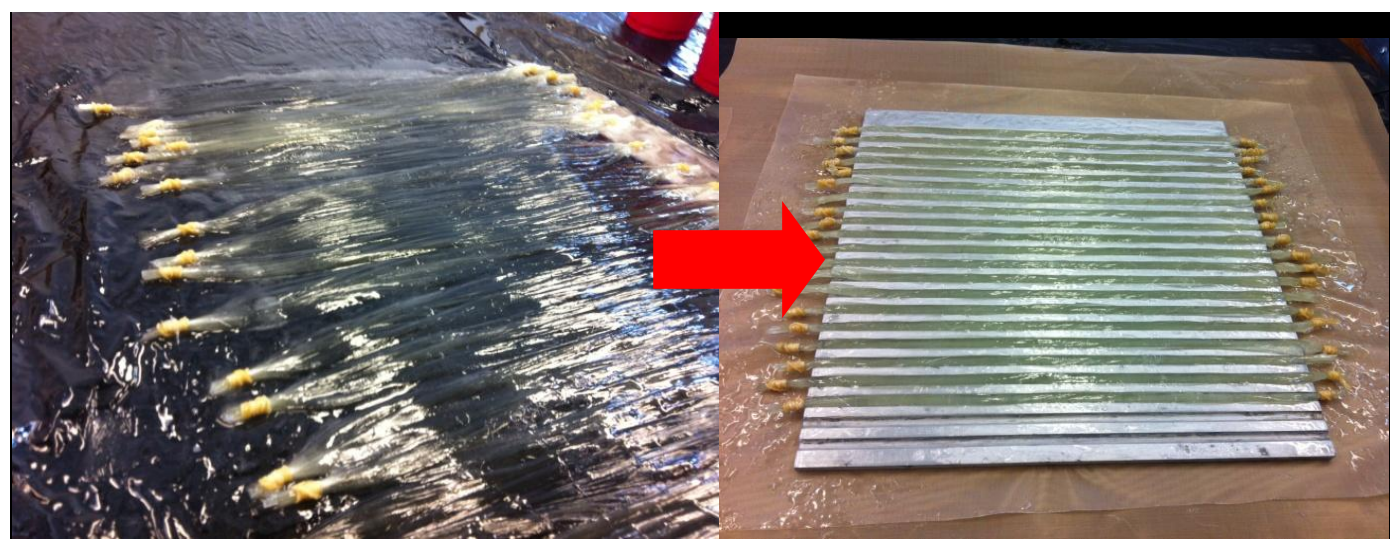

Figure 2.20. Hand Lay-up Method Used to Fabricate DAD Keys.

As shown in fig. 2.21, non-porous material was placed under the plate. This was done to prevent the resin from leaking onto surrounding surfaces. A separate sheet of non-porous material was placed on top of the plate, followed by another aluminum plate. A total weight of $200-\mathrm{lb}$ was then added on top of the plate to ensure the complete compacting of the fiberglass into the mold as can be seen in fig. 2.21 . 


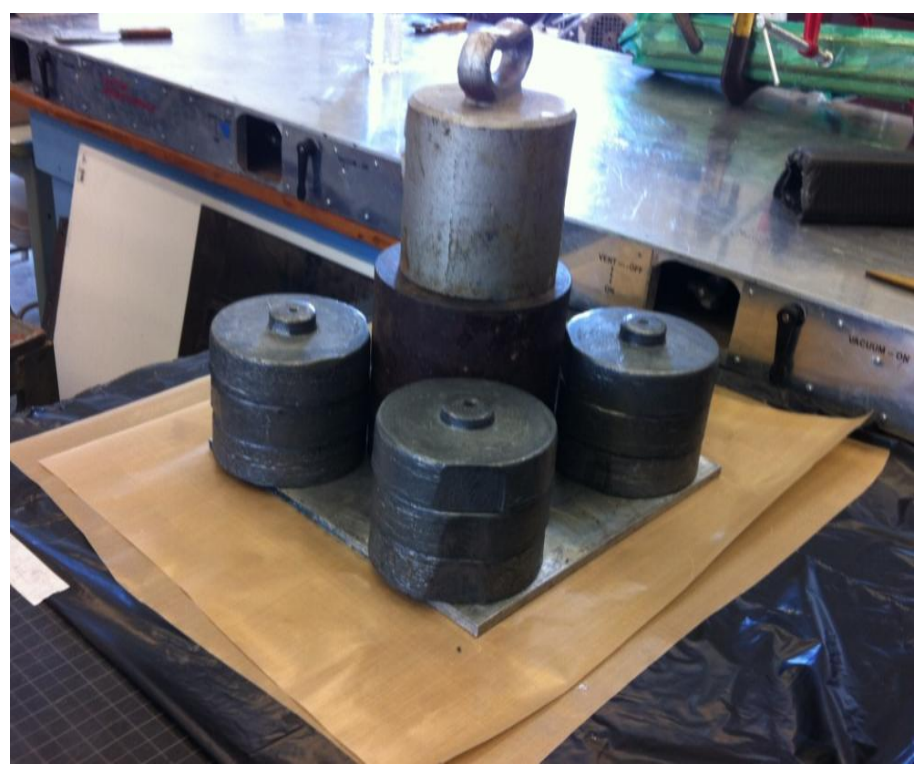

Figure 2.21. 200-lb of weight used to compress fiberglass DADs into mold.

After a 24 hour cure time, the weights were removed and the fiberglass DADs were ready to be removed. To remove the DADs from the mold, a sander was used to sand down the fiberglass until they were all separated by the aluminum as seen in fig.2.22. The final DADs were 12-inches long with a semicircular radius of 0.125 inches as shown in fig. 2.23.

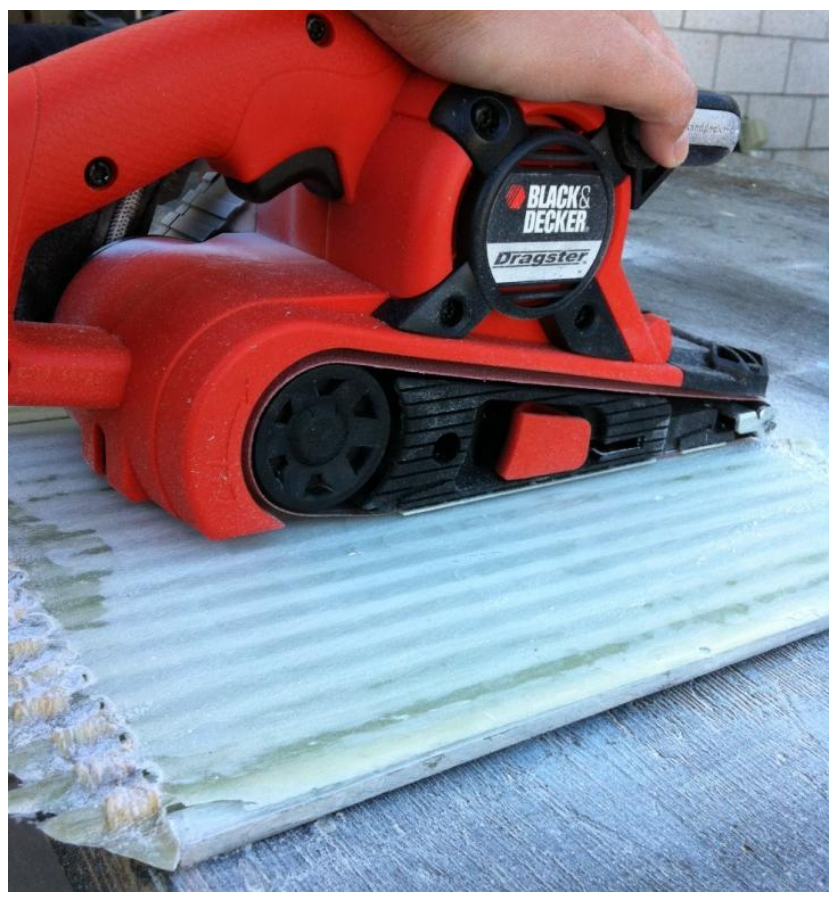

Figure 2.22. DADs are sanded down until separated from aluminum mold 


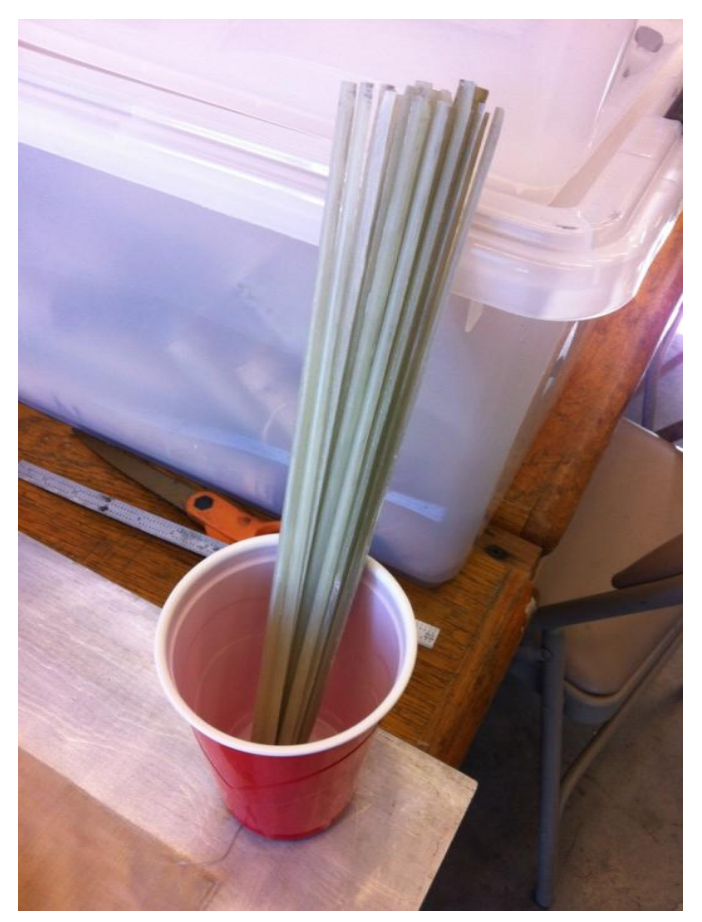

Figure 2.23. Cured DADs separated from the aluminum mold

\subsection{Composite Sandwich Beams}

Six beam cases were investigated in this research and were subjected to static and fatigue 4-point bend testing. The cases consisted of the following: a control with no initial delamination and no DAD, a beam with no delamination and two DADs placed transversely, a beam with no delamination and one DAD placed longitudinally, an initially delaminated beam, an initially delaminated beam with two DADs placed transversely, and lastly an initially delaminated beam with one DAD placed longitudinally. The initially delaminated beams included a 1 -inch long initial delamination, centrally located on the beam. The longitudinal DAD cases consisted of a beam with one DAD that was placed longitudinally and ran along the entire length of the beam. Several 12-inch by 12-inch composite sandwich plates were manufactured and cured using the autoclave, which followed the same method described in section 2.1. All test specimens manufactured consisted of two face sheets, each consisting of two layers of Advanced Composites Group LTM45EL/CF1803 pre-impregnated carbon fiber, and foam. From these composite sandwich plates, test specimen beams were cut to size using a wet tile saw. Each sub 
section will discuss the manufacturing method for each test case, including the implementation of the DAD keys and the method used for a forced delamination into the structure.

\subsubsection{Control Beam}

Two composite sandwich plates were manufactured for the control test specimen case. The control case consisted of beams with no initial delamination and no added DAD. The autoclave curing method was chosen as the curing method, again, because of its ability to produce uniform and nearly flawless test specimens and to keep the manufacturing methods consistent. This case was comprised of the Advanced Composites Group LTM45EL/CF1803 carbon fiber and General Plastics FR 6710 LastaFoam. Two layers of pre-impregnated carbon fiber were used to compose each face sheet while the core was made with one sheet of 0.5 -inch thick General Plastics FR 6710 LastaFoam polyurethane foam. To prepare the specimen for curing, four 12 -inch by 12 -inch pre-impregnated squares were carefully cut using a utility knife, followed by obtaining a pre-cut 12 -inch by 12 -inch foam sheet. Two sheets of porous material were cut into 14 -inch by 14 -inch squares using scissors, followed by cutting one 14 -inch by 14 inch square of breather material, and one 20 -inch by 20 -inch square of vacuum bag material was cut to contain the part. The layup sequence of materials was as follows: vacuum bag, breather material, porous material, two layers of bi-directional LTM45EL/CF1803 carbon fiber, FR 6710 LastaFoam, two layers of bi-directional LTM45EL/CF1803 carbon fiber, porous material, and nonporous material. A schematic of the stacking sequence can be seen in fig. 2.24 , where a sealant tape material is used to create a tight seal between the vacuum bag and aluminum plate. The porous material is used to prevent the excess resin from leaking out in the autoclave and the nonporous and breather material is used to provide a nice finish to the surfaces of the carbon fiber. 


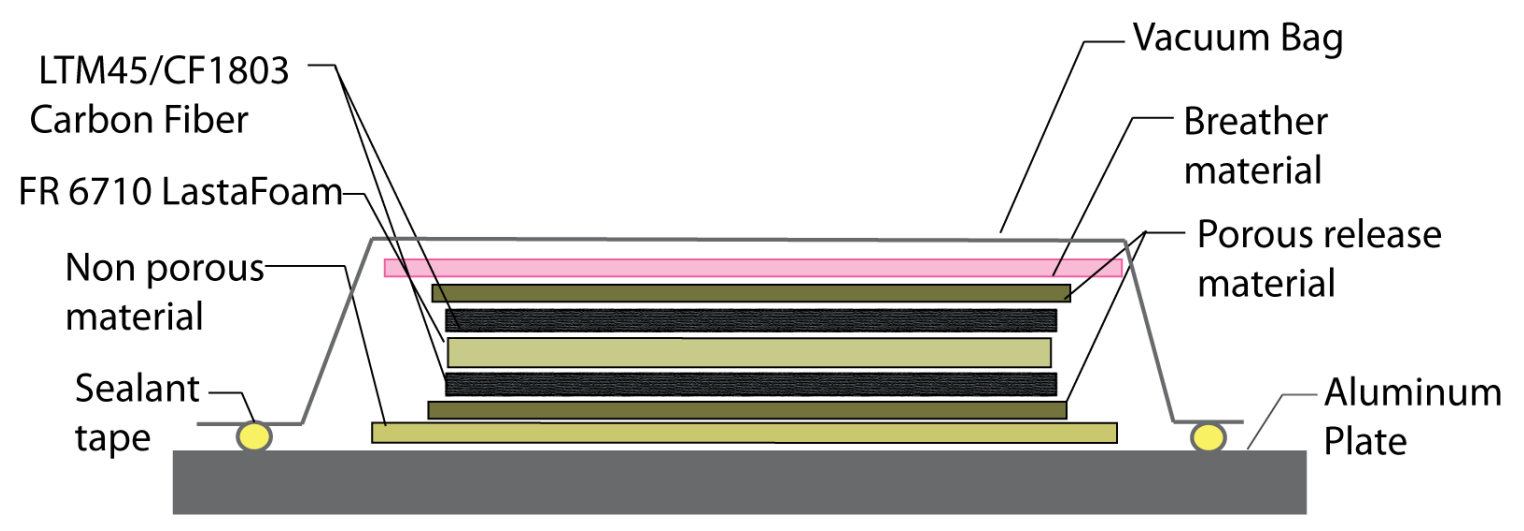

Figure 2.24. Layup schematic of composite sandwich plate for curing in the autoclave.

This layup must be placed on an aluminum plate that has been waxed using a high temp wax to prevent resin from hardening on the plate, as discussed previously. This provides a clean plate for the next cure and also provides a more uniform surface on the plate. The vacuum attachment was placed on the plate, and required a 3-inch by 3 -inch square piece of cotton, followed by placing a similar cotton piece over the holes in the aluminum plate. This was done to provide a perfect vacuum between the cured part and the atmosphere. The vacuum attachment was screwed into place by cutting a small hole in the vacuum bag. Following this procedure, the composite sandwich lay-up and aluminum plate were attached to the external vacuum and placed in the autoclave. The curing cycle employed for the composite sandwich test specimens followed the same curing cycle as the carbon fiber face sheet test coupons discussed in section 2.1 and instructions for the use of the autoclave can be found in Appendix A.

Following the cure cycle discussed in section 2.1, test specimen beams were cut to size from each composite sandwich plate using a wet tile saw. In total, twenty test specimen beams were manufactured for this case, ten specimens for 4-point bending tests and ten test specimens for fatigue testing. Figure 2.25 shows a drawing with dimensions of the control case beam with no DAD keys or initial delamination. 


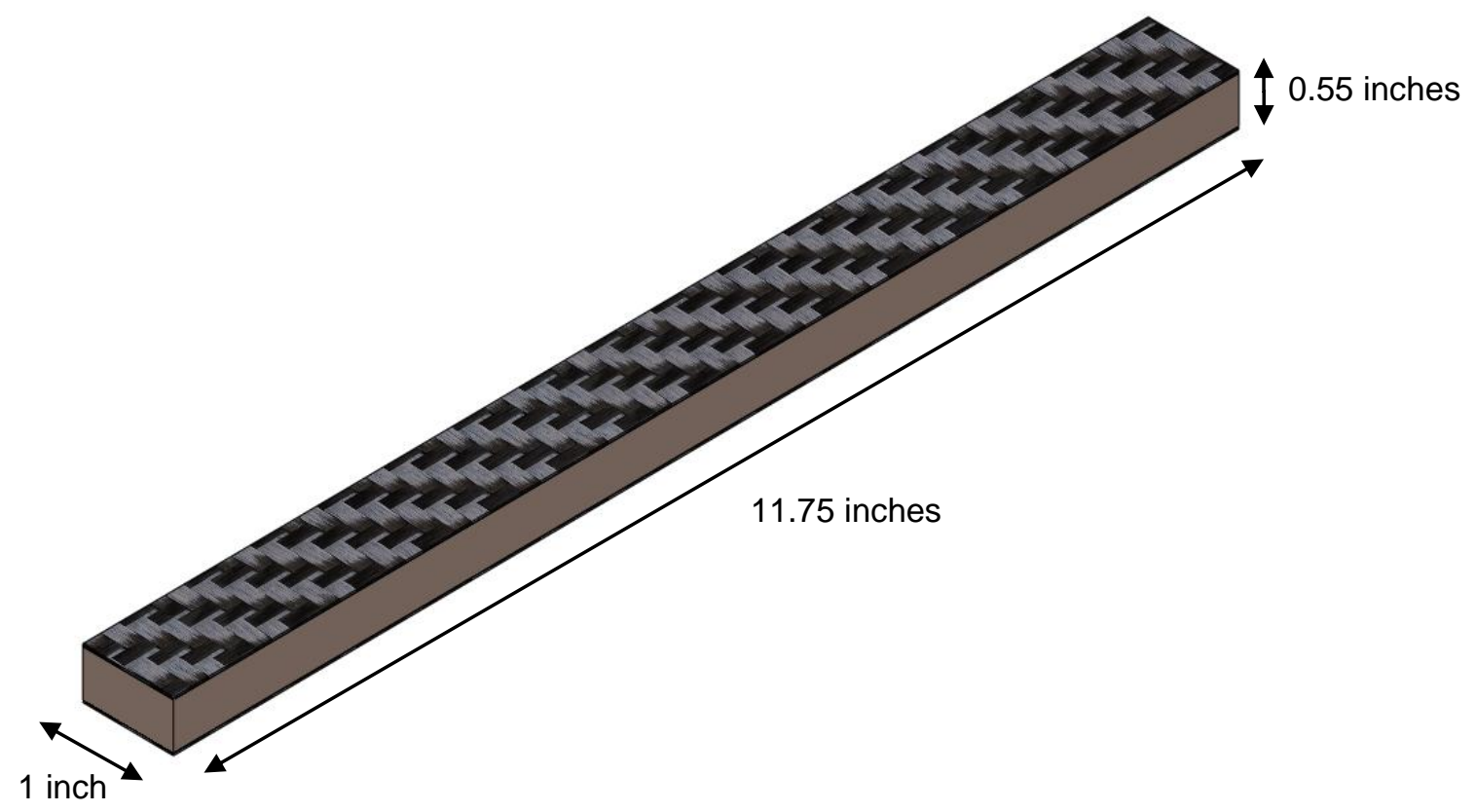

Figure 2.25. Drawing of a control case beam with no DAD keys or initial delamination.

\subsubsection{Beam with Transverse DADs}

Two composite sandwich plates were manufactured for the test specimen case with no initial delamination and transverse DAD keys. This case consisted of beams with no initial delamination and two DAD keys placed transversely in the center of the plate, 1 -inch apart. This case was comprised of the Advanced Composites Group LTM45EL/CF1803 carbon fiber, General Plastics FR 6710 LastaFoam, and two manufactured DADs as discussed in section 2.3. The layup sequence was similar to the control case with no initial delamination and no added DADs, except the foam was cut to accept the added DADs. In order for the foam core to accept the DAD keys, two 0.125 -inch radius half cylinder slots were milled in the foam using a CNC mill located in the Cal Poly Industrial and Manufacturing Engineering Machine Shop. The slots were milled so that a 1 -inch gap existed between them. The foam was cut to dimensions of 12 -inch by 12-inch, totaling two foam squares. With the prepared foam sheets, two cured DADs were placed inside the milled foam sheet and secured using a mixture of AEROPOXY PR2032 Epoxy Resin and PH3660 Epoxy Hardener, as shown in fig. 2.26 and fig. 2.27. 


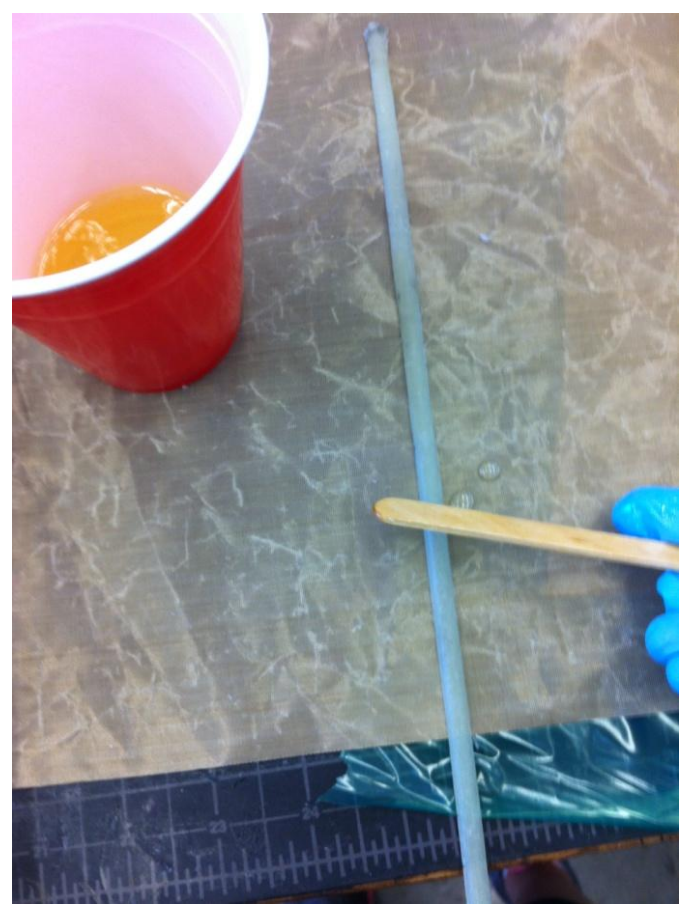

Figure 2.26. Epoxy resin/hardener applied to DADs used for secure bond in foam.

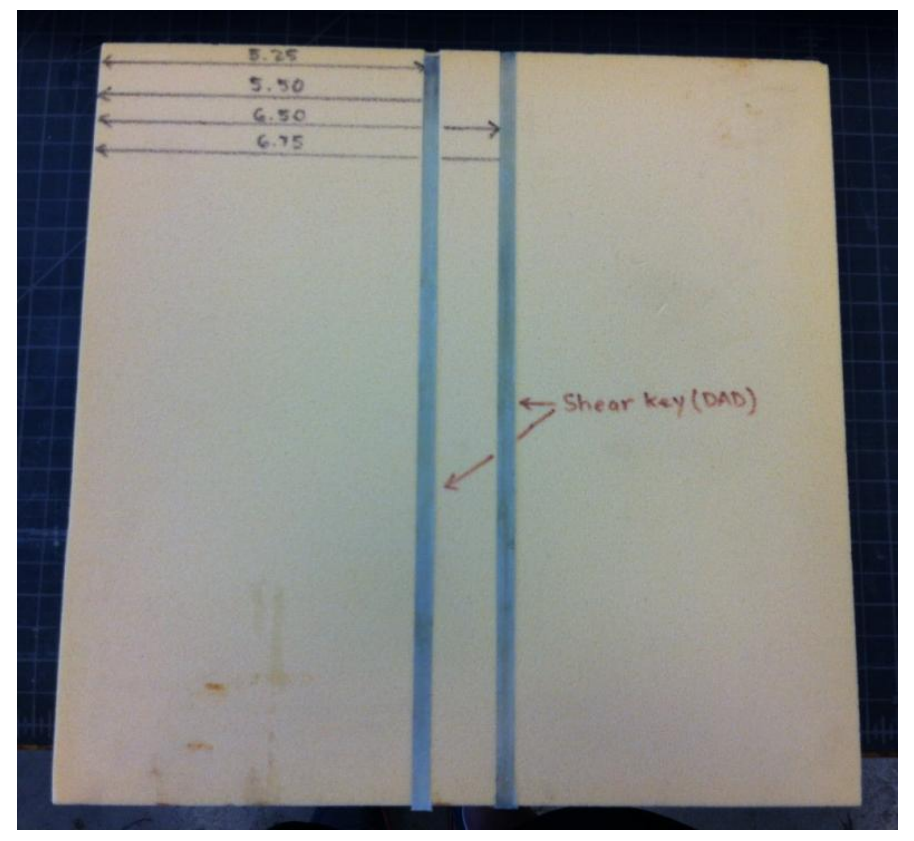

Figure 2.27. Milled foam sheet with DADs placed transversely (dimensions in inches).

Similar to the previous case, the layup sequence is as follows: vacuum bag, breather material, porous material, two layers of bi-directional LTM45EL/CF1803 carbon fiber, pre-milled FR 6710 LastaFoam, two layers of bi-directional LTM45EL/CF1803 carbon fiber, porous material, 
and non-porous material. The stacking sequence can be seen in fig. 2.28 , where a sealant tape is used and placed around the perimeter of the aluminum plate to create a tight seal between the vacuum bag and aluminum plate.

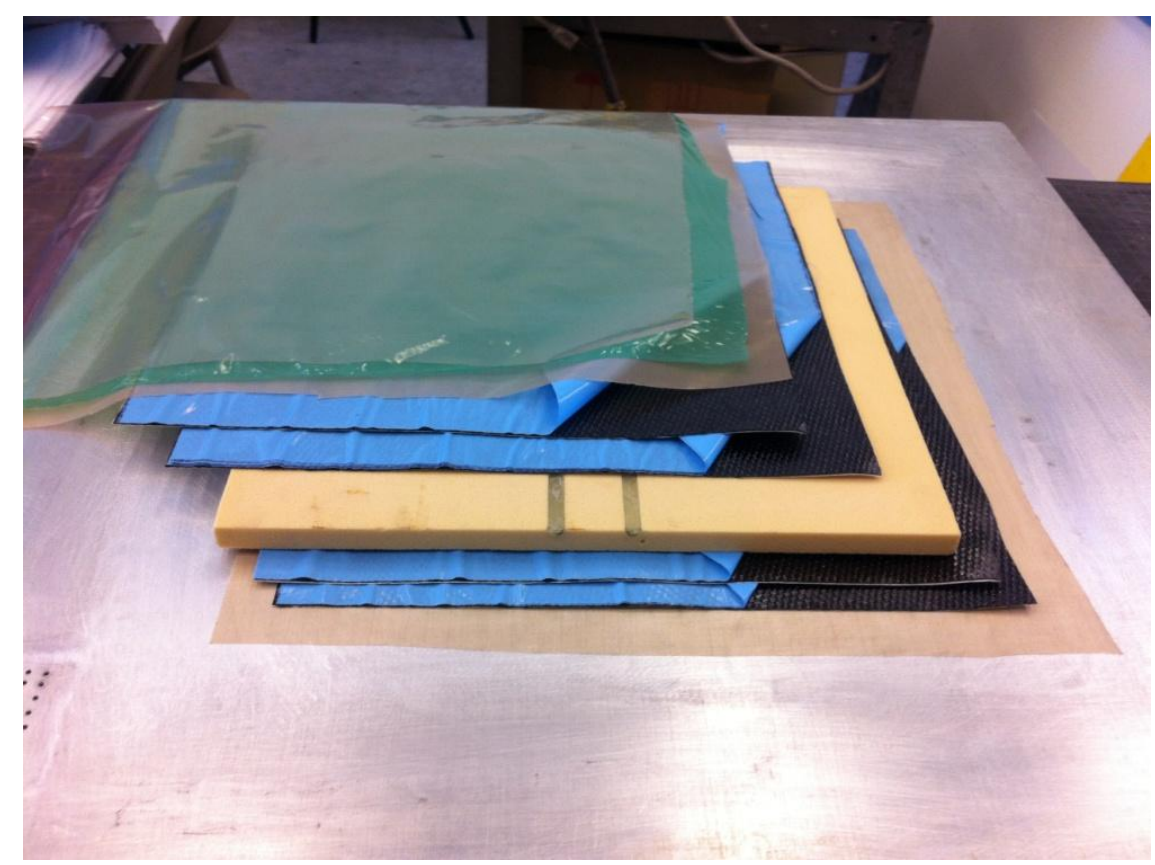

Figure 2.28. Layup sequence of composite sandwich plate with transverse keys ready for curing in the autoclave.

This layup was placed on an aluminum plate that had been waxed using a high temp wax, as stated in the previous section, and the vacuum attachment was placed on the plate with a 3-inch by 3-inch square piece of cotton. A similar cotton piece was placed over the holes in the aluminum plate. The vacuum attachment was screwed into place by cutting a small hole in the vacuum bag. The external vacuum was started before beginning the autoclave cure to create a tight vacuum for the composite sandwich plate as shown in fig. 2.29. The curing cycle in the autoclave began ten minutes after turning on the external vacuum. 


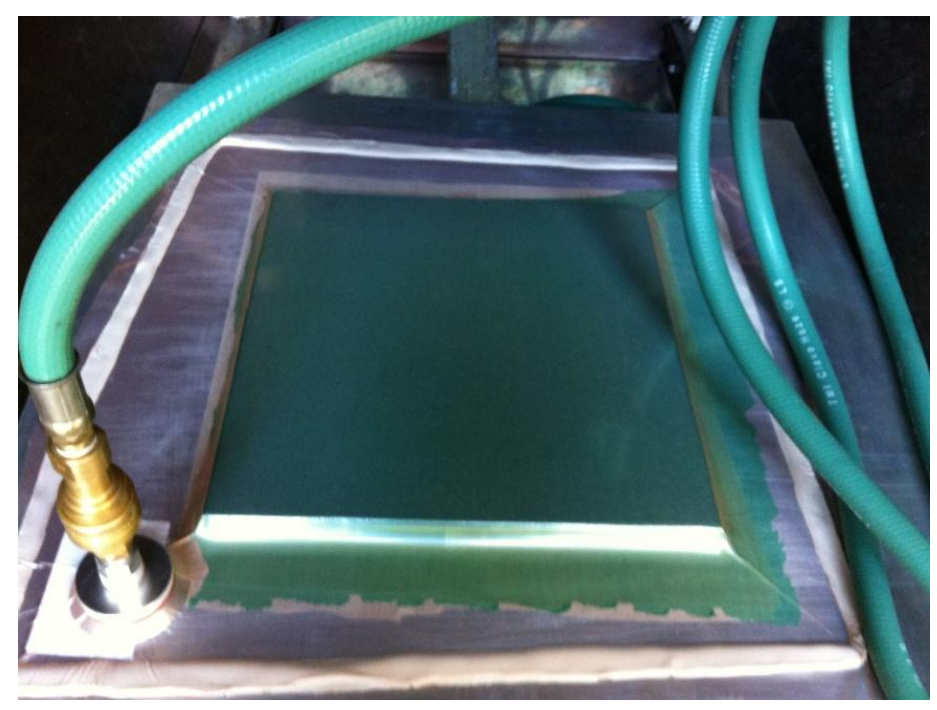

Figure 2.29. plate with no initial delamination and transverse DADs placed in the autoclave with tight vacuum.

Following the cure cycle discussed in section 2.1, test specimen beams were cut to size from each composite sandwich plate using a wet tile saw. In total, eighteen test specimen beams were manufactured for this case, eight specimens for 4-point bending tests and ten test specimens for fatigue testing. Figure 2.30 shows a drawing with dimensions of a beam with transverse DAD keys and no initial delamination.

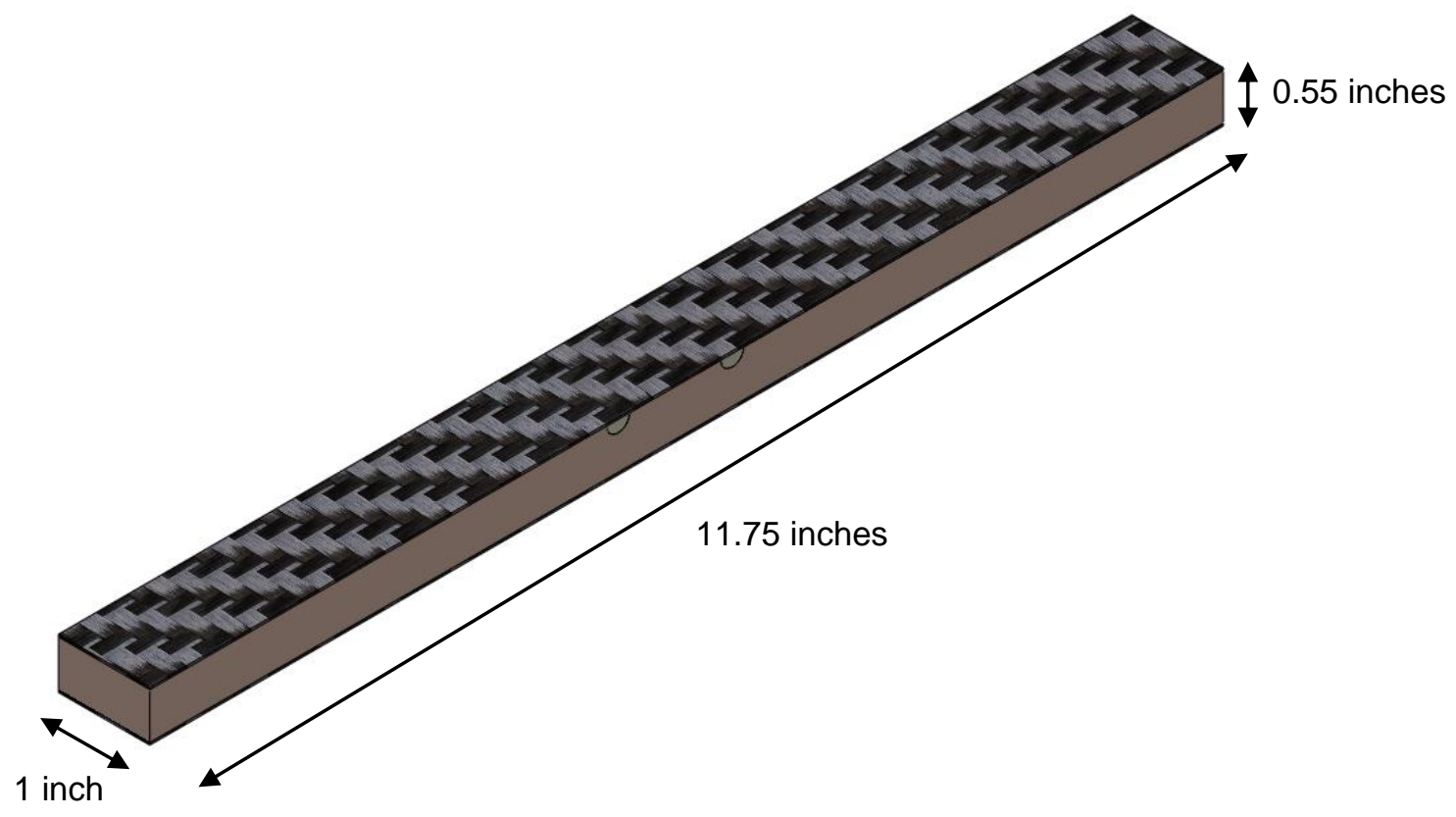

Figure 2.30. Drawing of a beam with transverse DAD keys and no initial delamination. 


\subsubsection{Beam with Longitudinal DAD}

Two composite sandwich plates were manufactured for the test specimen case with no initial delamination and a longitudinal DAD key. This control case consisted of beams with no initial delamination and one DAD key placed longitudinally along the length of the beam. This case was comprised of the Advanced Composites Group LTM45EL/CF1803 carbon fiber, General Plastics FR 6710 LastaFoam, and one manufactured DAD as discussed in section 2.3. The layup sequence was similar to the control case with no initial delamination or added DADs, except the foam was cut to accept the added DADs.

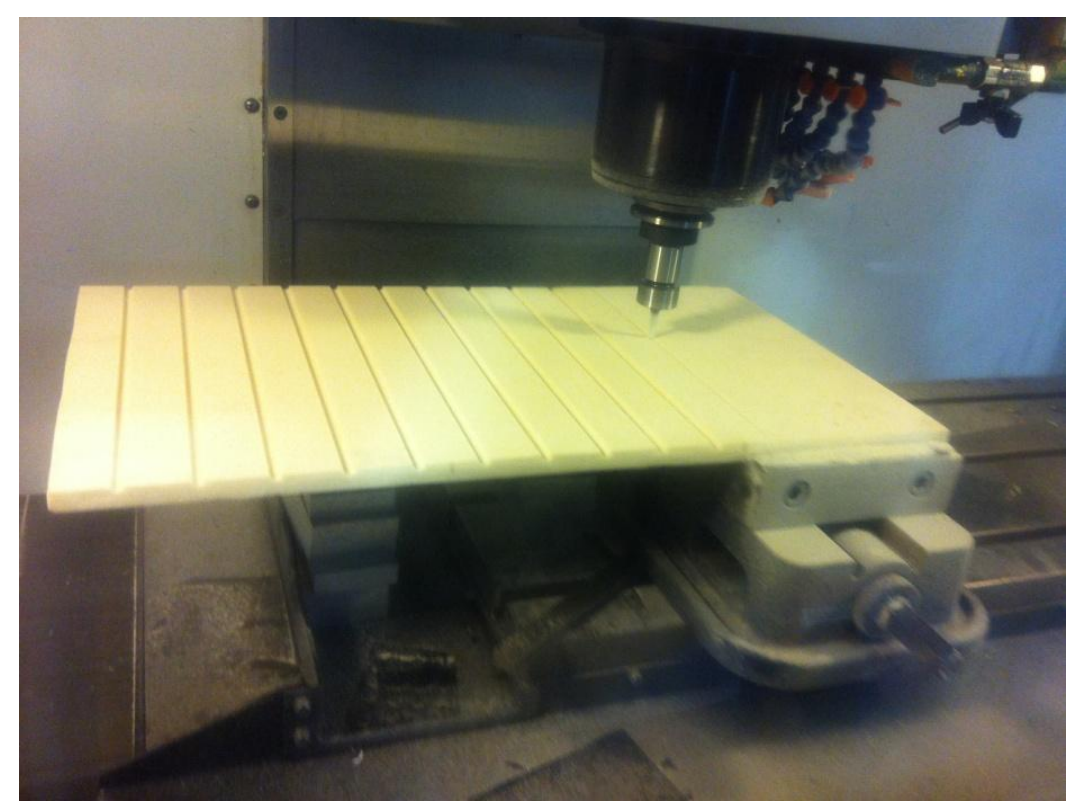

Figure 2.31. CNC machine milling slots in polyurethane foam to accept DAD keys.

In order for the foam core to accept the DAD keys, several 0.125 -inch radius half cylinder slots were milled in the foam using a CNC mill located in the Cal Poly Industrial and Manufacturing Engineering Machine Shop, as depicted in fig. 2.31. The slots were milled such that a 2.5-inch gap existed between them so there was enough tolerance when cutting the plate into test specimens. The foam was cut to dimensions of 12 -inch by 12 -inch after milling the slots which totaled to two foam squares. With the prepared foam sheets, a cured DAD was placed inside each slot and secured using a mixture of AEROPOXY PR2032 Epoxy Resin and PH3660 Epoxy Hardener, as shown in fig. 2.32. 


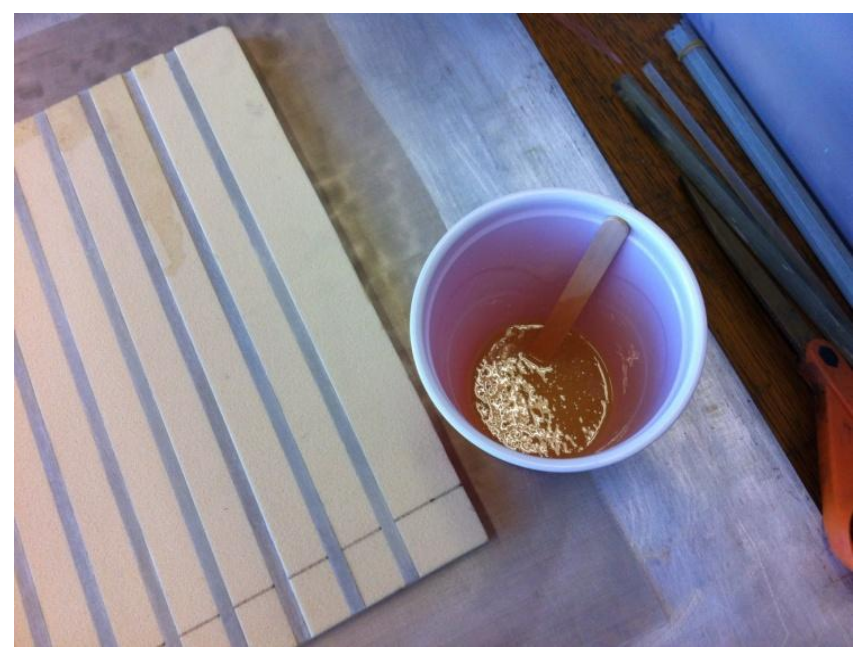

Figure 2.32. Milled foam sheet with DADs placed longitudinally.

Similar to the previous case, the layup sequence is as follows: vacuum bag, breather material, porous material, two layers of bi-directional LTM45EL/CF1803 carbon fiber, pre-milled FR 6710 LastaFoam, two layers of bi-directional LTM45EL/CF1803 carbon fiber, porous material, and non-porous material. The stacking sequence can be seen in fig. 2.33, where a sealant tape is used placed around the perimeter of the aluminum plate to create a tight seal between the vacuum bag and aluminum plate.

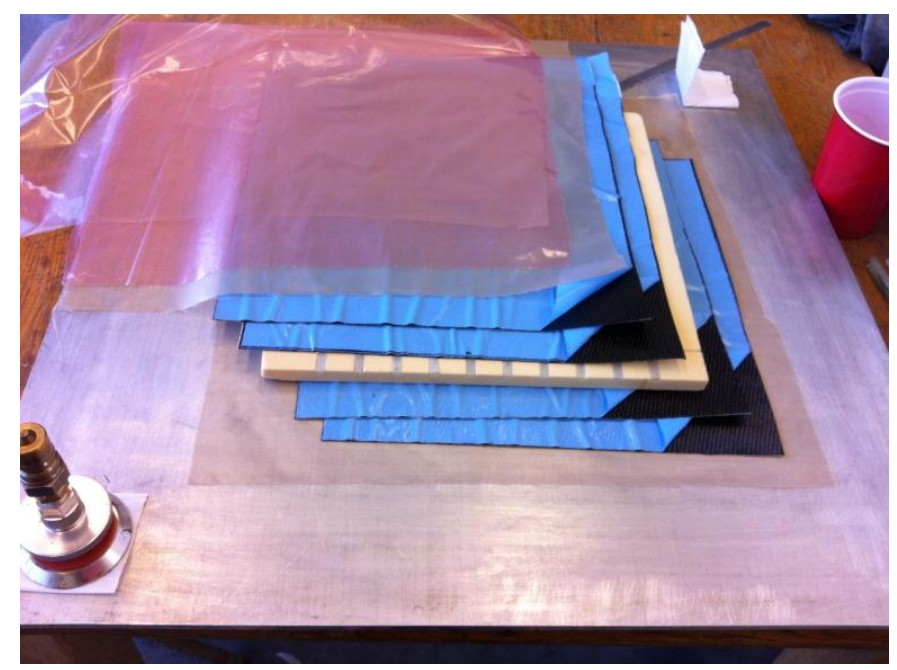

Figure 2.33. Layup sequence of composite sandwich plate with longitudinal keys ready for curing in the autoclave.

This layup was placed on an aluminum plate that had been waxed using a high temp wax and the vacuum attachment was placed on the plate with a 3 -inch by 3 -inch square piece of cotton. A similar cotton piece was placed over the holes in the aluminum plate. The vacuum 
attachment was screwed into place by cutting a small hole in the vacuum bag. The external vacuum was started before beginning the autoclave cure in order to create a tight vacuum for the composite sandwich lay-up. The curing cycle, discussed in section 2.1, began ten minutes after turning on the external vacuum.

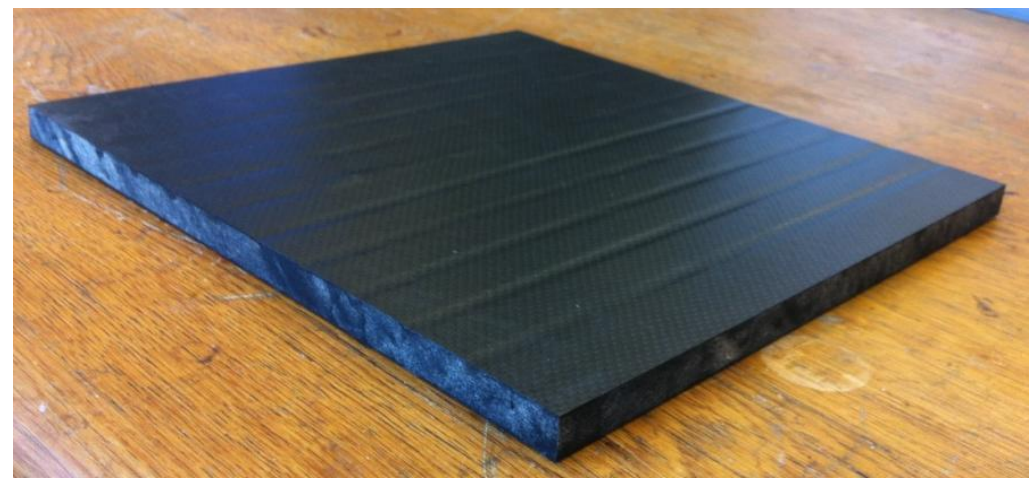

Figure 2.34. Cured composite sandwich plate with no initial delamination and longitudinal keys.

The resulting plate, shown in fig. 2.34, was cut into 10 test specimen beams using a wet tile saw. In total, sixteen test specimen beams were manufactured for this case, six specimens for 4-point bending tests and ten test specimens for fatigue testing. Figure 2.35 and 2.36 show both the dimensions and configuration of a beam with a longitudinal DAD key and no initial delamination.

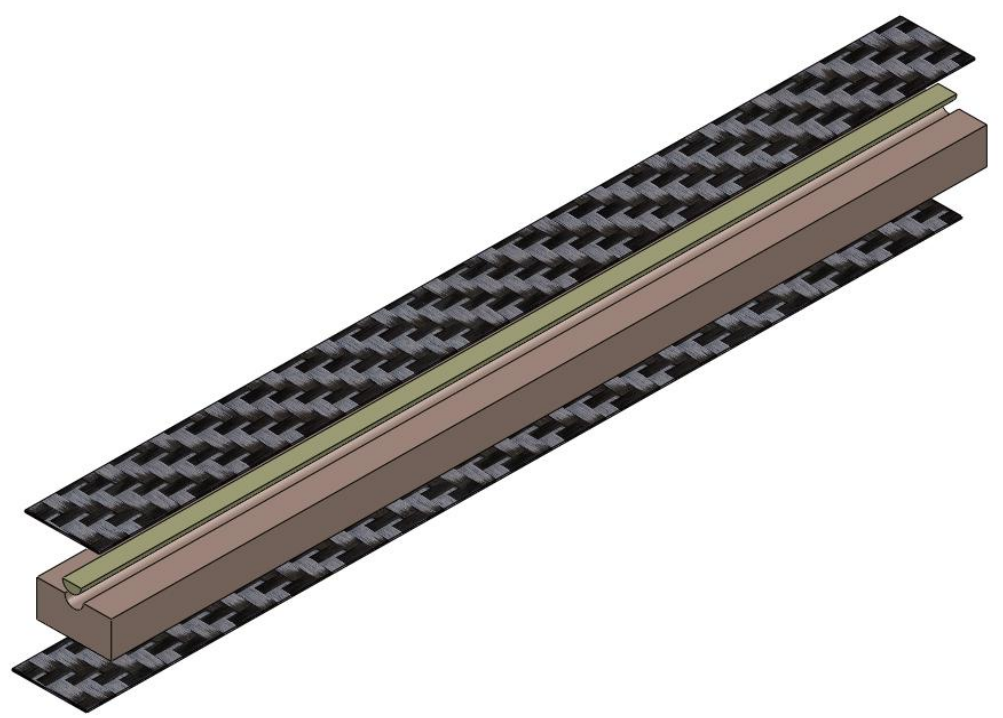

Figure 2.35. Configuration of beam with longitudinal DAD key and no delamination. 


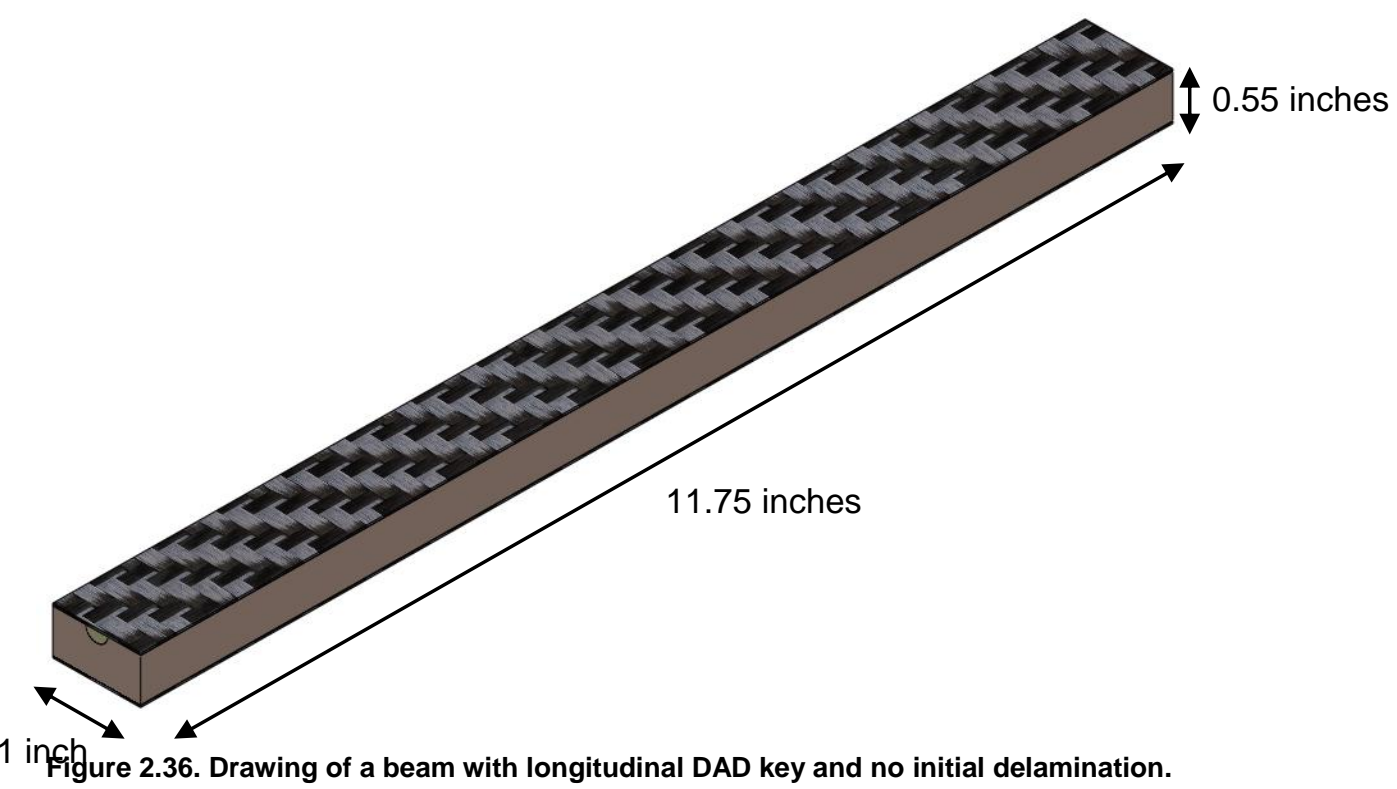

\subsubsection{Initially Delaminated Beam}

Two composite sandwich plates with a centrally located forced delamination were manufactured for the case with an initial delamination and no added DAD. The autoclave curing method was used as the curing method to keep the manufacturing methods consistent. This case was comprised of the Advanced Composites Group LTM45EL/CF1803 carbon fiber, General Plastics FR 6710 LastaFoam, and a non-porous strip. Two layers of pre-impregnated carbon fiber were used to compose each face sheet while the core was made with one sheet of 0.5 -inch thick General Plastics FR 6710 LastaFoam polyurethane foam. To force the delamination, a 1-inch by 14-inch strip was placed centrally on the foam core, as shown in fig. 2.37 .

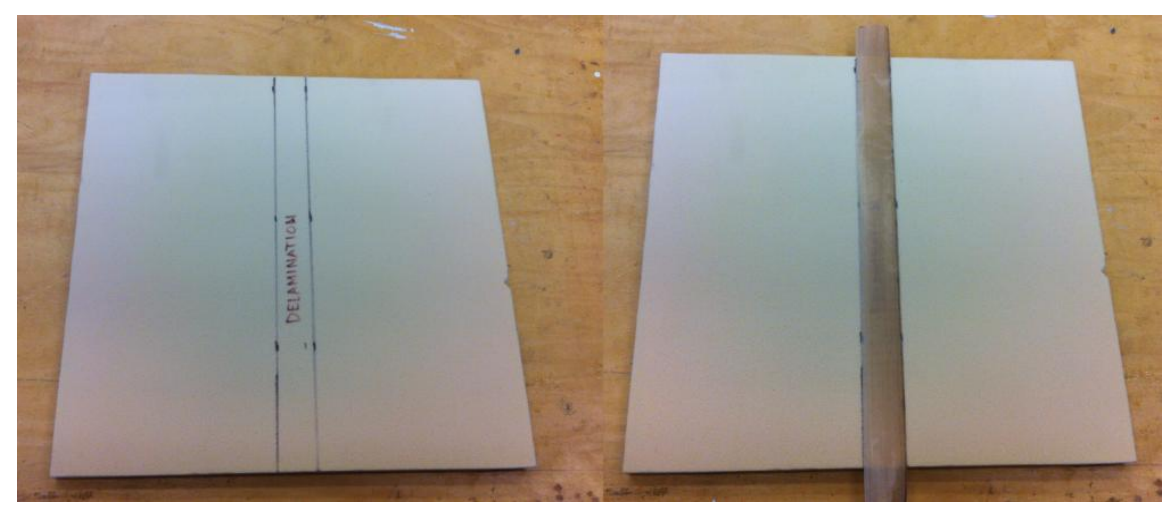

Figure 2.37. Foam core with sketched delamination placement (left) and non-porous strip placed centrally (right). 
To prepare the specimen for curing, four 12 -inch by 12 -inch pre-impregnated squares were cut, followed by obtaining a pre-cut 12 -inch by 12 -inch foam sheet. Two sheets of porous material were cut into 14 -inch by 14 -inch squares using scissors, followed by cutting one 14-inch by 14 -inch square of breather material, one 14 -inch by 14 -inch non-porous square, and one precut 1 -inch by 14 -inch non-porous strip, and one 20 -inch by 20 -inch square of vacuum bag material was cut in order to contain the part. The layup sequence of materials was as follows: vacuum bag, breather material, porous material, two layers of bi-directional LTM45EL/CF1803 carbon fiber, one 1-inch by 14-inch non-porous strip, one sheet of FR 6710 LastaFoam, two layers of bi-directional LTM45EL/CF1803 carbon fiber, porous material, and non-porous material. The stacking sequence can be seen in fig. 2.38 , where a sealant tape material is used to create a tight vacuum seal between the vacuum bag and the aluminum plate.

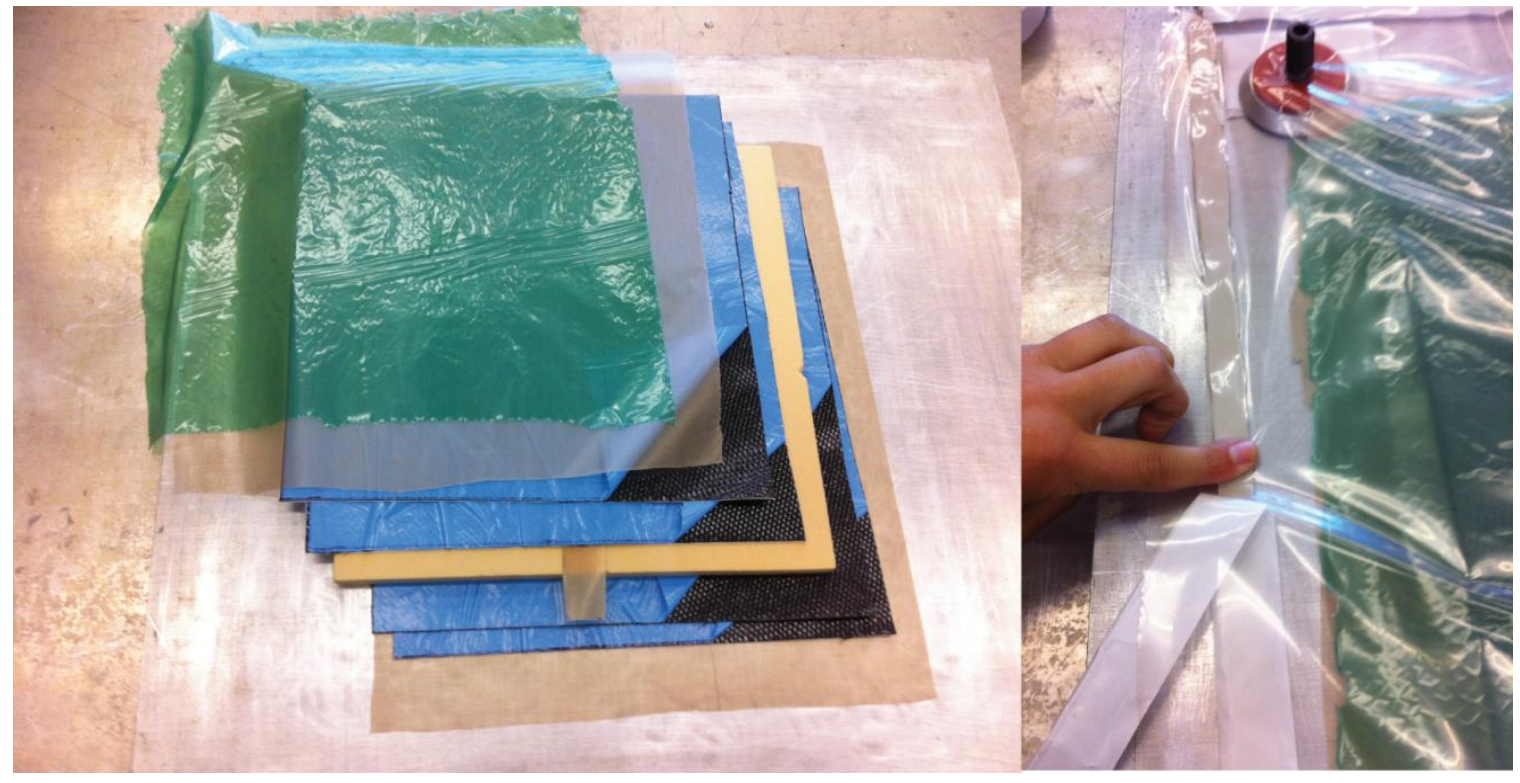

Figure 2.38. Layup of composite sandwich plate with initial delamination and sealant tape placement for curing in the autoclave.

This layup was placed on an aluminum plate that had been waxed using a high temp wax, where a vacuum attachment was placed on the plate, and required a 3 -inch by 3 -inch square piece of cotton, followed by placing a similar cotton piece over the holes in the aluminum plate. The test piece and aluminum plate were then placed in the autoclave and the curing cycle followed the same curing cycle as the carbon fiber face sheet test coupons discussed in section 2.1. 
Following the cure cycle, the test specimen beams were cut to size from each manufactured composite sandwich plate using a wet tile saw. In total, twenty test specimen beams were manufactured for this case, ten specimens for 4-point bending tests and ten test specimens for fatigue testing. Figure 2.39 shows a drawing of a beam with no DAD keys and a 1 -inch centrally located initial delamination, where it follows the same dimensions as the previous cases.

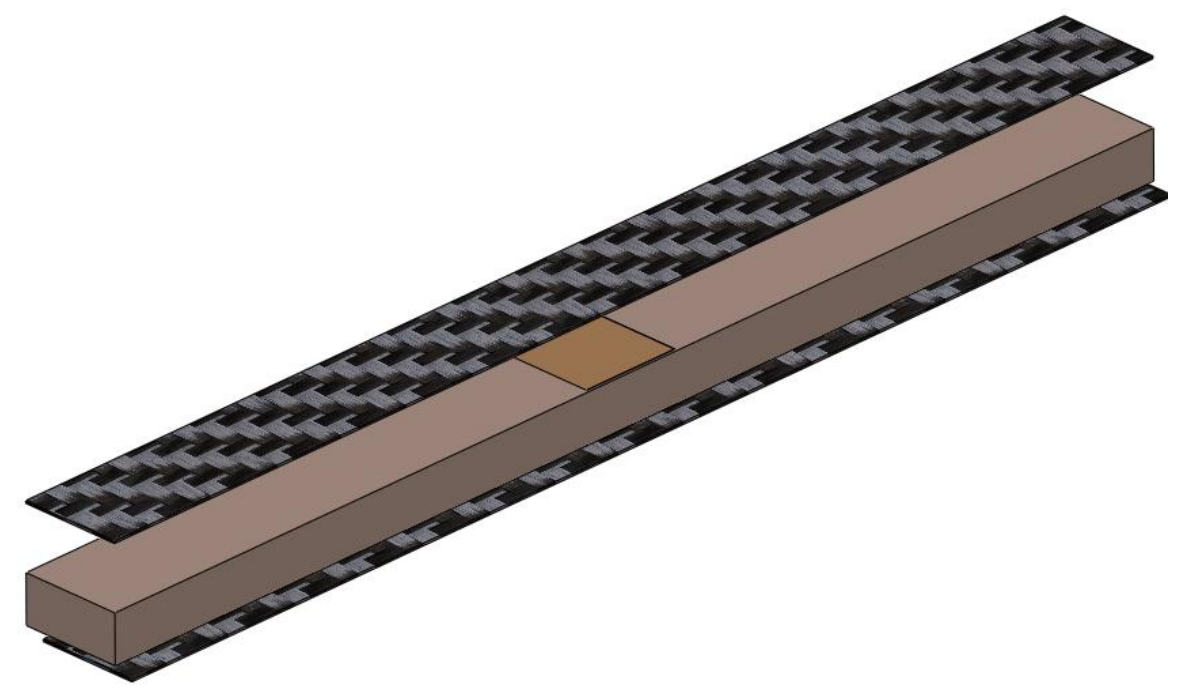

Figure 2.39. Drawing of a beam with initial delamination and no DADs.

\subsubsection{Initially Delaminated Beam with Transverse DADs}

Two composite sandwich plates were manufactured for the test specimen case with a 1inch centrally located initial delamination and transverse DAD keys. This delaminated case consisted of beams with an initial delamination and two DAD keys placed transversely in the center of the beam, 1-inch apart, on either side of the delamination. This case was comprised of the Advanced Composites Group LTM45EL/CF1803 carbon fiber, General Plastics FR 6710 LastaFoam, two manufactured DADs, and a non-porous strip. The lay-up sequence was similar to the beam case with no initial delamination and transversely placed DADs, except a 1 -inch by 14inch non-porous strip was added. Using the pre-cut foam sheets, a non-porous strip was placed on top of the foam along with two cured DADs that were placed inside the milled foam sheet and secured using a mixture of AEROPOXY PR2032 Epoxy Resin and PH3660 Epoxy Hardener, as shown in fig. 2.40 . 


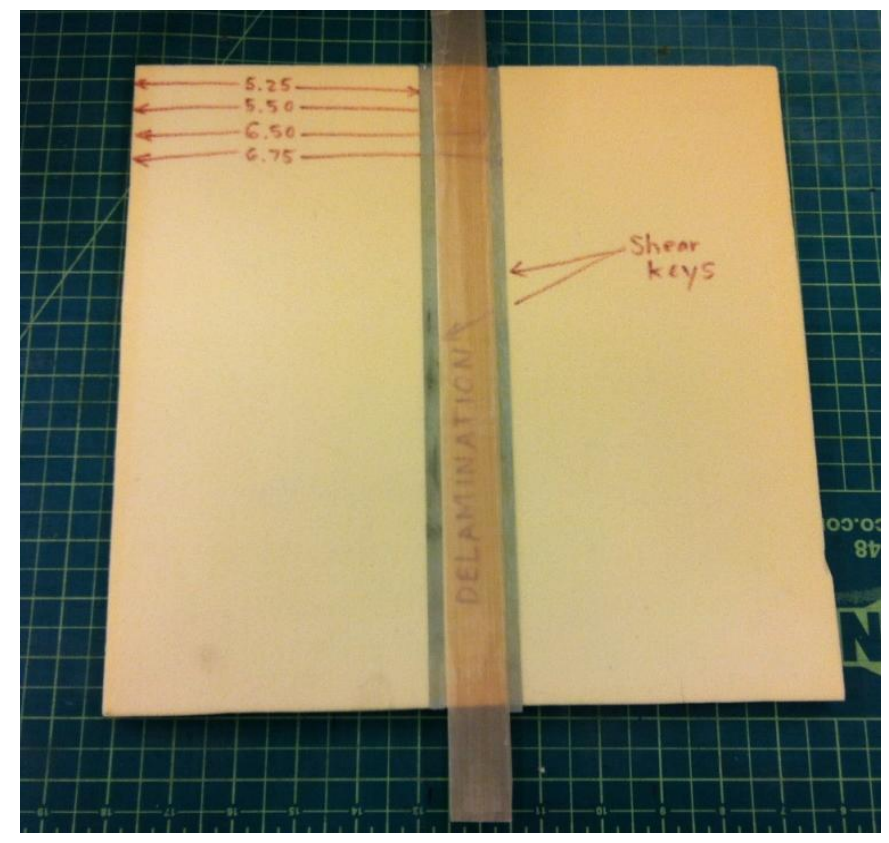

Figure 2.40. pre-cut foam sheet with DADs placed transversely and non-porous strip to force a delamination (dimensions in inches).

Similar to the previous cases, the layup sequence is as follows: vacuum bag, breather material, porous material, two layers of bi-directional LTM45EL/CF1803 carbon fiber, non-porous strip, pre-milled FR 6710 LastaFoam, two layers of bi-directional LTM45EL/CF1803 carbon fiber, porous material, and non-porous material. The stacking sequence was nearly identical to the case with no initial delamination and added transverse DADs, but the strip of non-porous material is added to create an initial delamination to the plate.

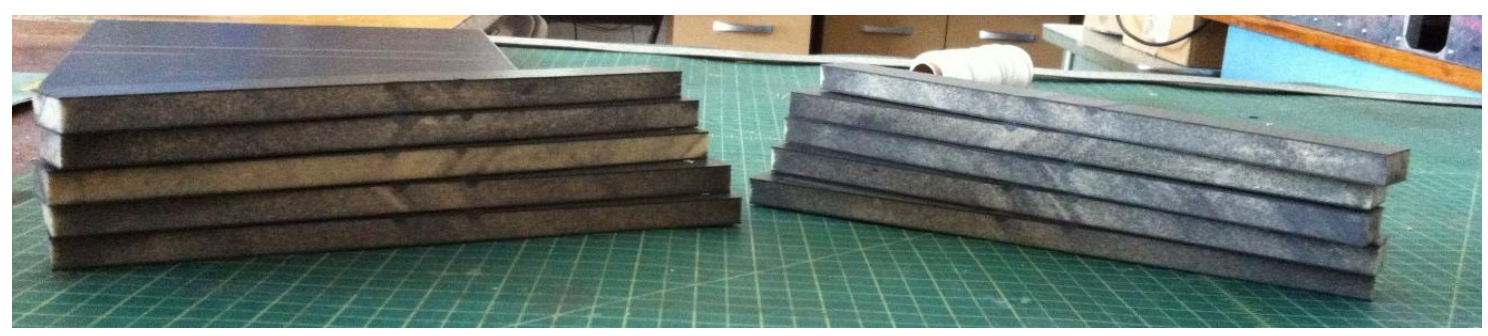

Figure 2.41. Test specimen beams with DADs placed transversely and initial delamination cut from cured plates.

The completed layup was placed on an aluminum plate and placed in the autoclave for curing. Following the cure cycle discussed in section 2.1, test specimen beams were cut to size, shown in fig. 2.41, from each composite sandwich plate using a wet tile saw. In total, twenty test specimen beams were manufactured for this case, ten specimens for 4-point bending tests and ten test specimens for fatigue testing. Figure 2.42 shows an exploded view of a beam with 
transverse DAD keys and an initial delamination, where the centrally located square represents the delamination in the beam. The dimensions are identical to the previous beams discussed.

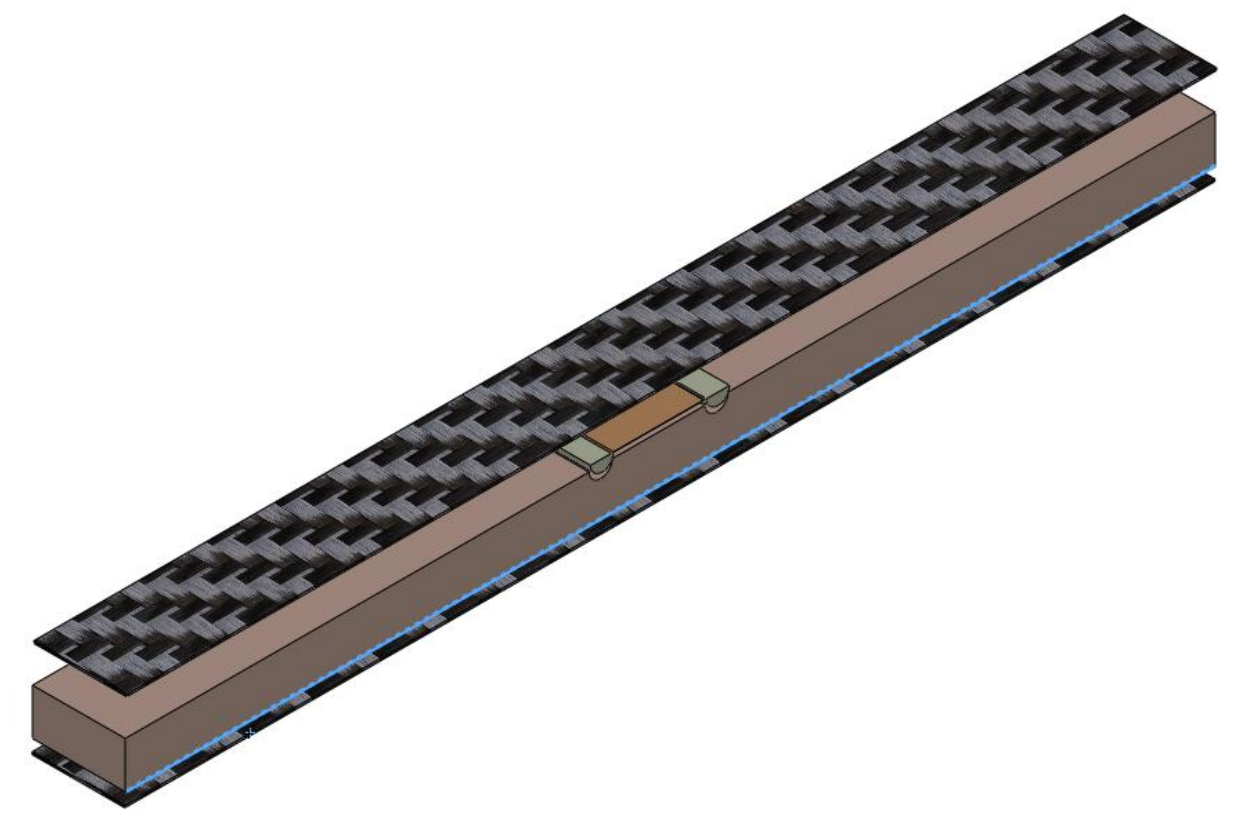

Figure 2.42. Exploded view of a beam with transverse DAD keys and a centrally located 1-inch initial delamination.

\subsubsection{Initially Delaminated Beam with Longitudinal DAD}

The final test case was a composite sandwich beam with a 1-inch centrally located delamination and an added longitudinal DAD key. Two composite sandwich plates were manufactured for this case where the DAD key was placed longitudinally along the length of the beam. This case was comprised of the Advanced Composites Group LTM45EL/CF1803 carbon fiber, General Plastics FR 6710 LastaFoam, a non-porous strip, and one manufactured DAD as discussed in section 2.3. The layup sequence was similar to the case with no initial delamination and a longitudinal $\mathrm{DAD}$, except the 1-inch wide non-porous material was added to force a delamination in the beams. With the pre-cut foam sheets, a cured DAD was placed inside each slot and secured using a mixture of AEROPOXY PR2032 Epoxy Resin and PH3660 Epoxy Hardener and a 1-inch wide non-porous strip was placed over the foam, as shown in fig. 2.43. 


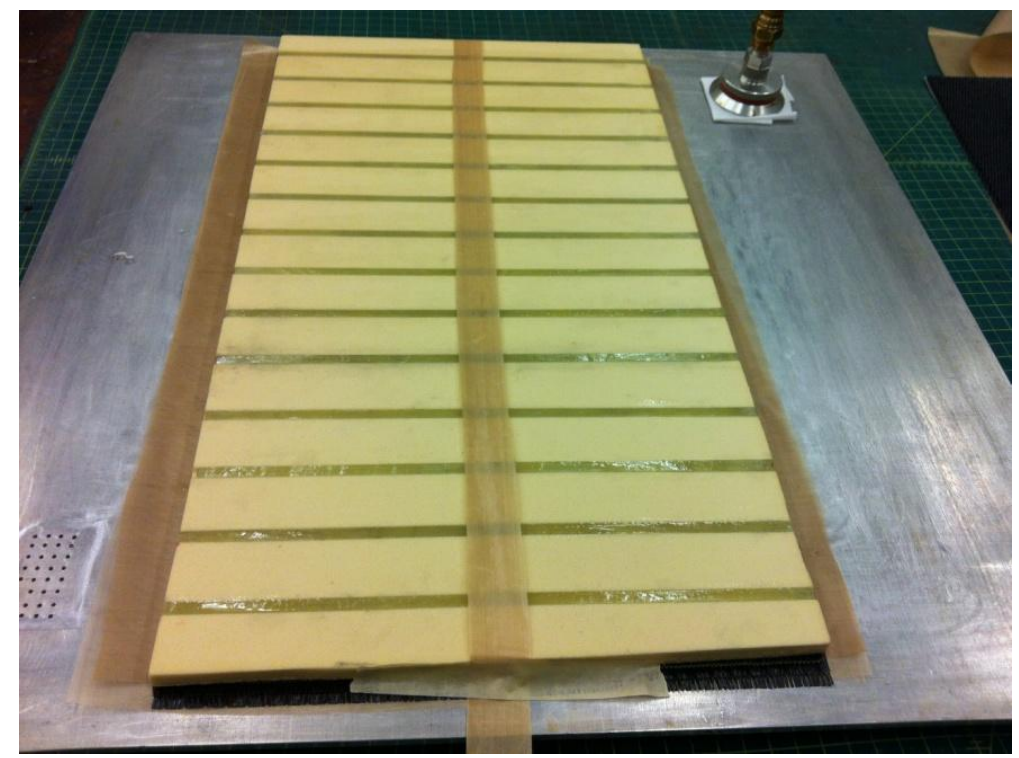

Figure 2.43. Pre-cut foam sheet with DADs placed longitudinally and added non-porous strip.

Similar to the previous cases, the layup sequence is as follows: vacuum bag, breather material, porous material, two layers of bi-directional LTM45EL/CF1803 carbon fiber, non-porous strip, pre-milled FR 6710 LastaFoam, two layers of bi-directional LTM45EL/CF1803 carbon fiber, porous material, and non-porous material. The stacking sequence can be seen in fig. 2.44 below.

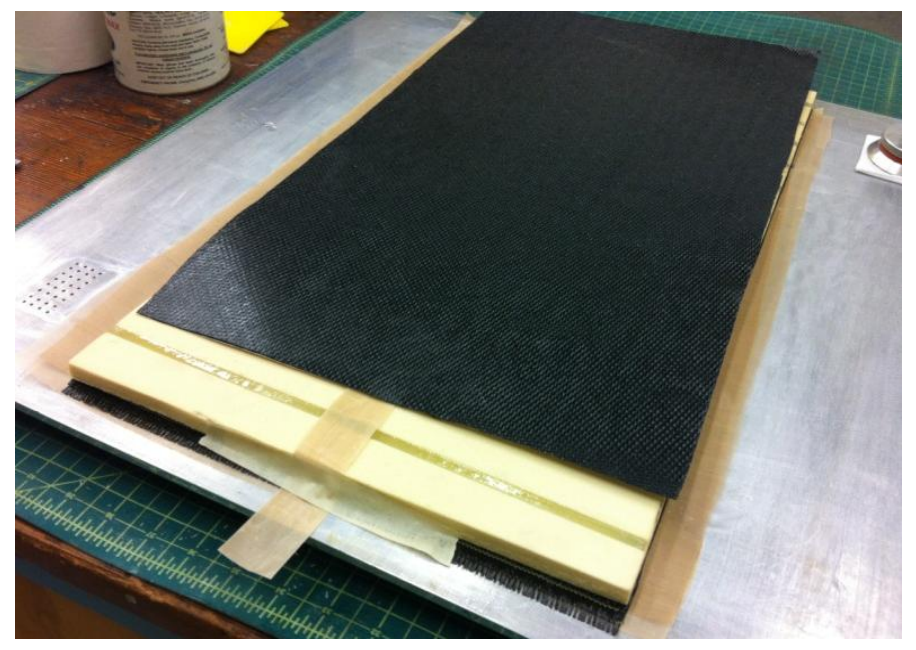

Figure 2.44. Layup sequence of composite sandwich plate with longitudinal keys and delamination ready for curing in the autoclave.

Like previous cases, the layup was placed in the autoclave under a vacuum seal and cured according to the cure cycle discussed in section 2.1., where the resulting plates were cut using a wet tile saw. In total, fifteen test specimen beams were manufactured for this case, five specimens for 4-point bending tests and ten test specimens for fatigue testing. Figure 2.45 shows 
an exploded view of a beam with a longitudinal DAD key and a 1-inch wide centrally located initial delamination. As stated before, the dimensions are identical to the previous beams discussed.

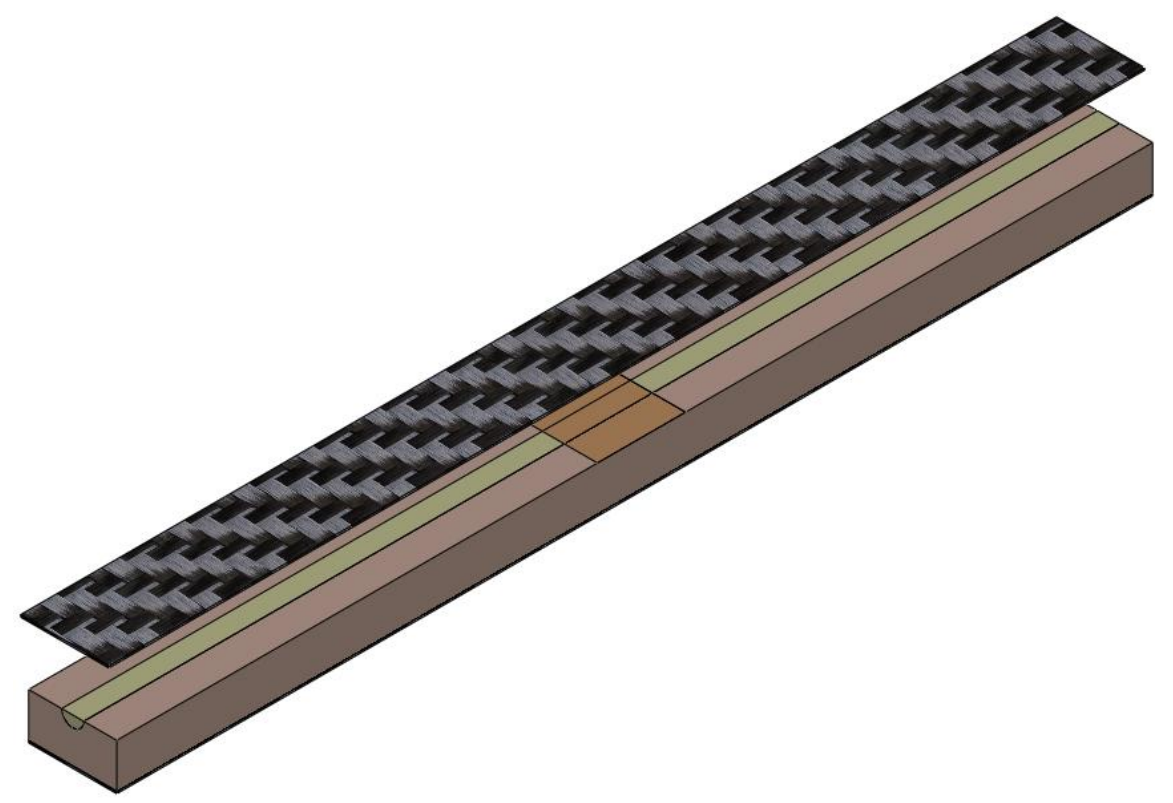

Figure 2.45. Exploded view of beam with longitudinal DAD key and initial delamination. 


\section{Chapter 3 Experimental Design Methodology}

This chapter discusses the experimental design methodology developed and the procedures followed for static 4-point bend testing, impact testing, and dynamic 4-point bend testing (fatigue). Static 4-point bend testing and impact testing followed specific ASTM test standards and a summary of the test methodology will also be presented. Trials and final design methodology for fatigue testing will be discussed in detail as well. First static 4-point bend testing was performed on the six composite sandwich beam cases. Thereafter, impact testing was performed on the control beam case and initially delaminated beam for subsequent static 4-point bend testing. Impact testing was performed on the non-delaminated beam cases and these three cases were fatigued under 4-point bend shortly after. Lastly, fatigue testing was performed on all six beam cases without any impact.

\subsection{Static 4-point Bend Test}

Static 4-point bend testing followed the ASTM D6272 standard test - "Standard Test Method for Flexural Properties of Unreinforced and Reinforced Plastics and Electrical Insulating Materials by Four-Point Bending" [27]. This particular test method required the use of a 4-point loading system that is simply supported on both ends of length of the test specimen. This test standard required a beam with a rectangular cross section, which is loaded by two point loads. The load and support spans were dictated by the specimen dimensions, where the support span distance was sixteen times the thickness of the test specimen and the load span was one third of the distance between the supports. A schematic of the 4-point bend test specifications is shown below in fig. 3.1. Following the ASTM test standard, it was determined that the support span and load span were 8.80 inches and 2.93 inches, respectively. 


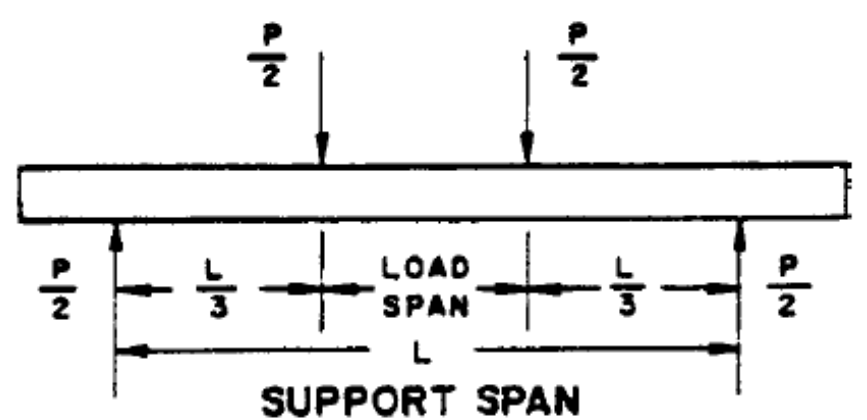

Figure 3.1. Schematic of 4-point bending test specifications followed by ASTM 6272 standard test [27].

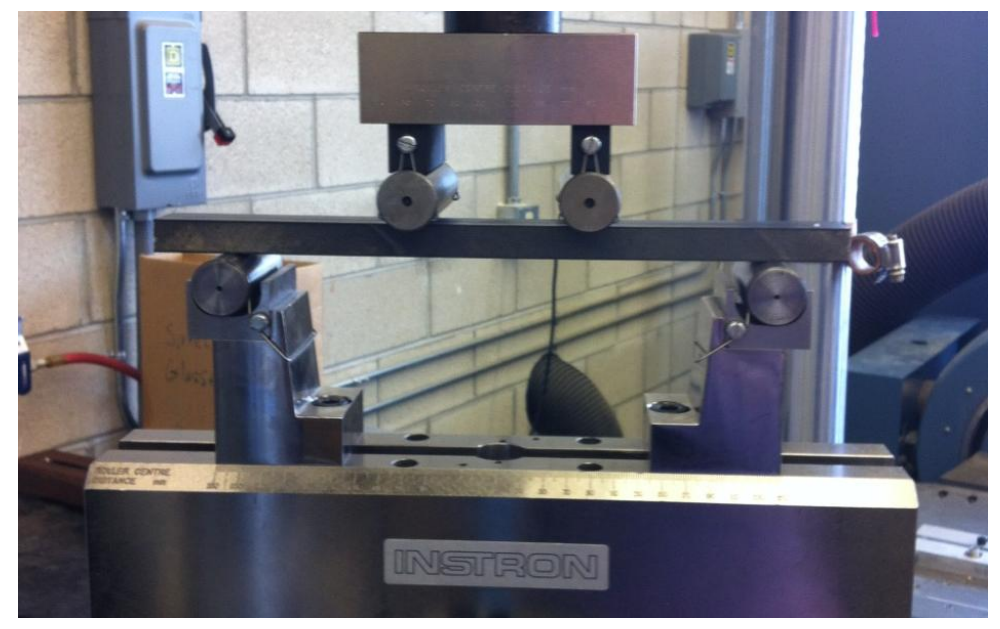

Figure 3.2. Instron 8801 Servohydraulic Fatigue Testing System used for 4-point bend testing.

The Instron 8801 Servohydraulic Fatigue Testing System in the Cal Poly Aerospace Structures/Composites laboratory was used to satisfy the test system specifications, illustrated with the test set up in fig. 3.2. Bluehill 2 software was used in conjunction with the Instron 8801 testing system, which requires an input of constant crosshead loading rate. To determine this value, the test standard gives a rate of crosshead motion $R$ as shown in eqn. 3.1.

$$
R=\frac{0.185 Z L^{2}}{d}
$$

where variable $L$ refers to the support span, $d$ refers to the depth of the beam, and $Z$ refers to the rate of straining of the outer fibers. Given that the rate of straining of the outer fibers $Z$ was equal to 0.01 , the crosshead rate was determined to be $0.369 \mathrm{in} / \mathrm{min}$ for all 4 -point bend tests. All test 
specimens were loaded until fracture of until $5 \%$ of the maximum fiber strain was reached, whichever came first. The Bluehill2 software and Instron 8801 machine recorded time, crosshead position, load, deflection, and strain. Of these test parameters, only the load and deflection values were used to determine important values such as ultimate strength.

\subsection{Impact Test}

Impact testing followed the ASTM D7136 standard test - "Standard Test Method for Measuring the Damage Resistance of a Fiber-Reinforced Polymer Matrix Composite to a DropWeight Impact Event" [28]. This standard was used and modified to obtain the impact resistance properties of the test specimen beams investigated in this research. In this test standard, test dimensions were to adhere to a 6 -inch by 4 -inch test specimen standard. However, in this research test dimensions 4 -point bend test specimens were 1 -inch wide by 11.75 -inches long. Therefore, the actual impact test specimen dimensions were driven by the static and dynamic 4point bend tests following an impact event. Further, the test standard called for the mass of the mass impactor to be $12-\mathrm{lb}_{\mathrm{m}}$, but with the available test machine used in this research, the actual mass impactor weight equated to $7.56-\mathrm{lb}_{\mathrm{m}}$. Lastly, the standard is geared towards unidirectional and woven laminates and does not specifically mention the use of composite sandwiches, but since the face sheets of the composite sandwich beams tested in this standard were laminates, this was deemed acceptable.

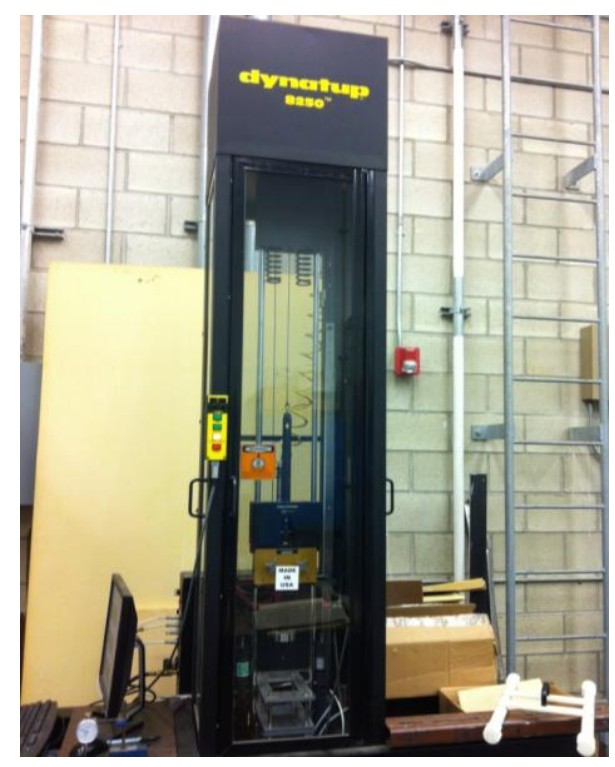


Figure 3.3. Dynatup 8250 drop weight impact machine used for impact testing.

The Dynatup 8250 drop weight impact machine located in the Cal Poly Aerospace Structures/Composites laboratory was used to complete impact testing, following the ASTM D7136 standard test. Figure 3.3 shows the Dynatup 8250 used for the impact testing. Thanks to the efforts of graduate student Kodi Rider, the use of the Dynatup 8250 impact machine was possible with the development of a full data acquisition system. With this data acquisition system the following parameters were obtained for each drop test: the instantaneous velocity of the impactor at the time of impact, a force vs. time history of the impact event, and acceleration vs. time history of the impacting crosshead throughout the impact event. Further, a test specimen support fixture was designed, per standard specifications, and manufactured by an undergraduate student Daniel Barath. A 3-inch wide by 5-inch long unsupported section was machined where the impacting tup was centrally located, as shown in fig. 3.4. The hemispherical impact tup used had a diameter of 0.625 -inches, following test standard specifications, and the hardness of the material was $62 \mathrm{HRC}$. In Kodi Rider's graduate research, problems arose with test specimen beams due to limited contact with support fixture, leading to several rebounds from the impactor tup. Therefore, two plates manufactured by Cody Thompson were used, where a 0.75 -inch wide by 1.5 -inch long unsupported section was machined for the area of impact to the beam, shown in fig. 3.5 .

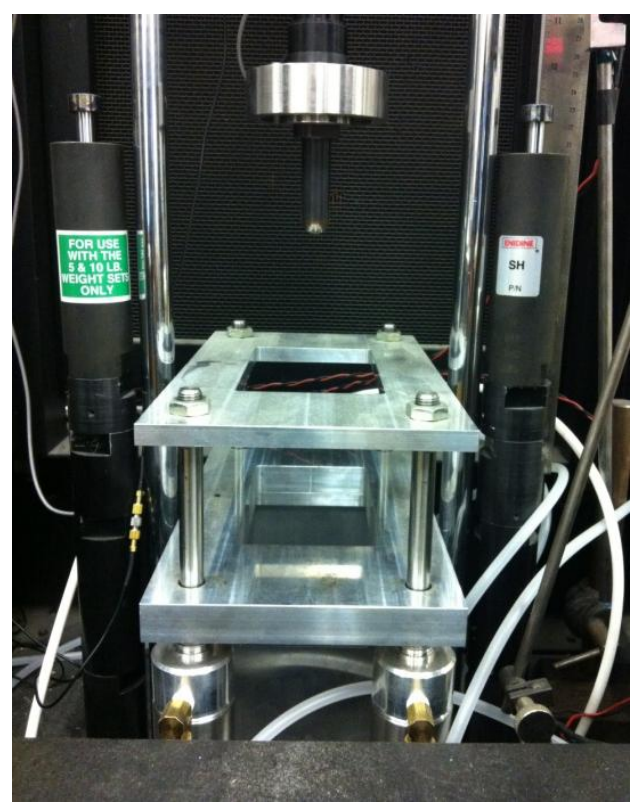


Figure 3.4. Test support fixture used for impact tests.

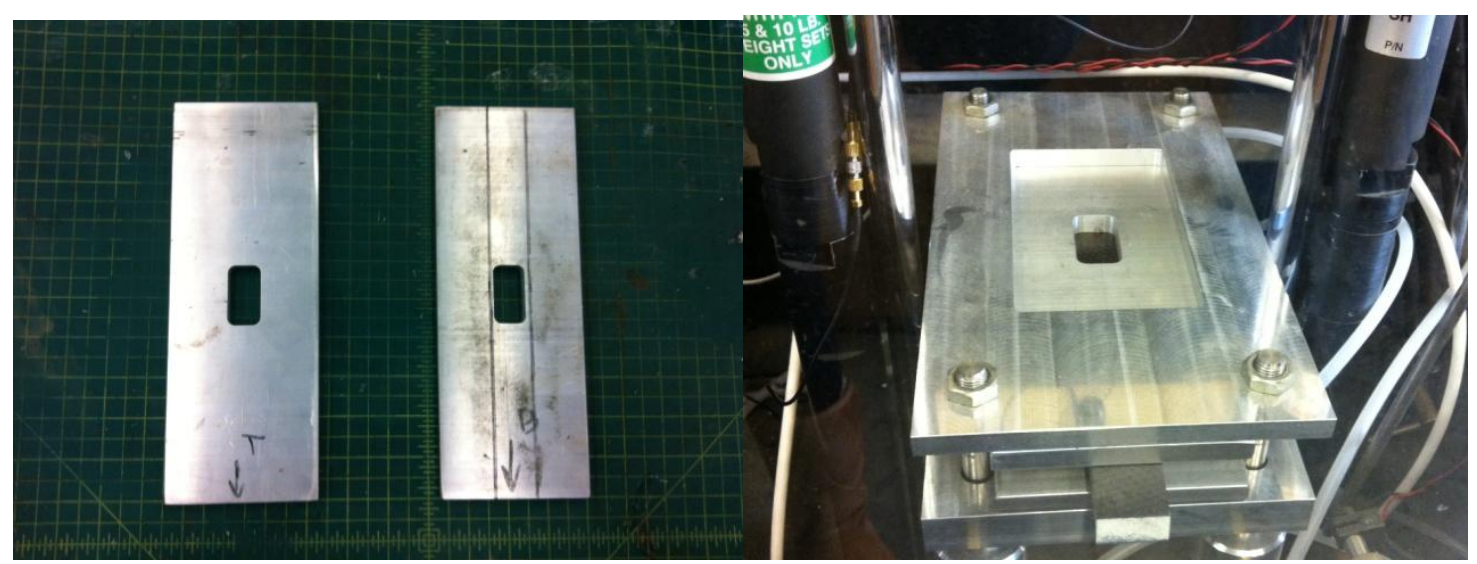

Figure 3.5. Two added support plates for clamping test beams in support fixture.

Real time data of the Si detector data, accelerometer data, and load cell data were shown on the Dynatup DAQ software window, as shown in fig. 3.6. Here the user was able to turn on the laser in the software and start/stop the data acquisition system. Once the test was run and the stop button was pressed, the file saved as a .Ivm file which was read into a MATLAB graphical user interface script. This GUI, shown in fig. 3.7, allowed for easy access to results of the impact test including impact force vs. time plot, acceleration of the tup vs. time plot, instantaneous velocity at impact, and maximum impact force. 


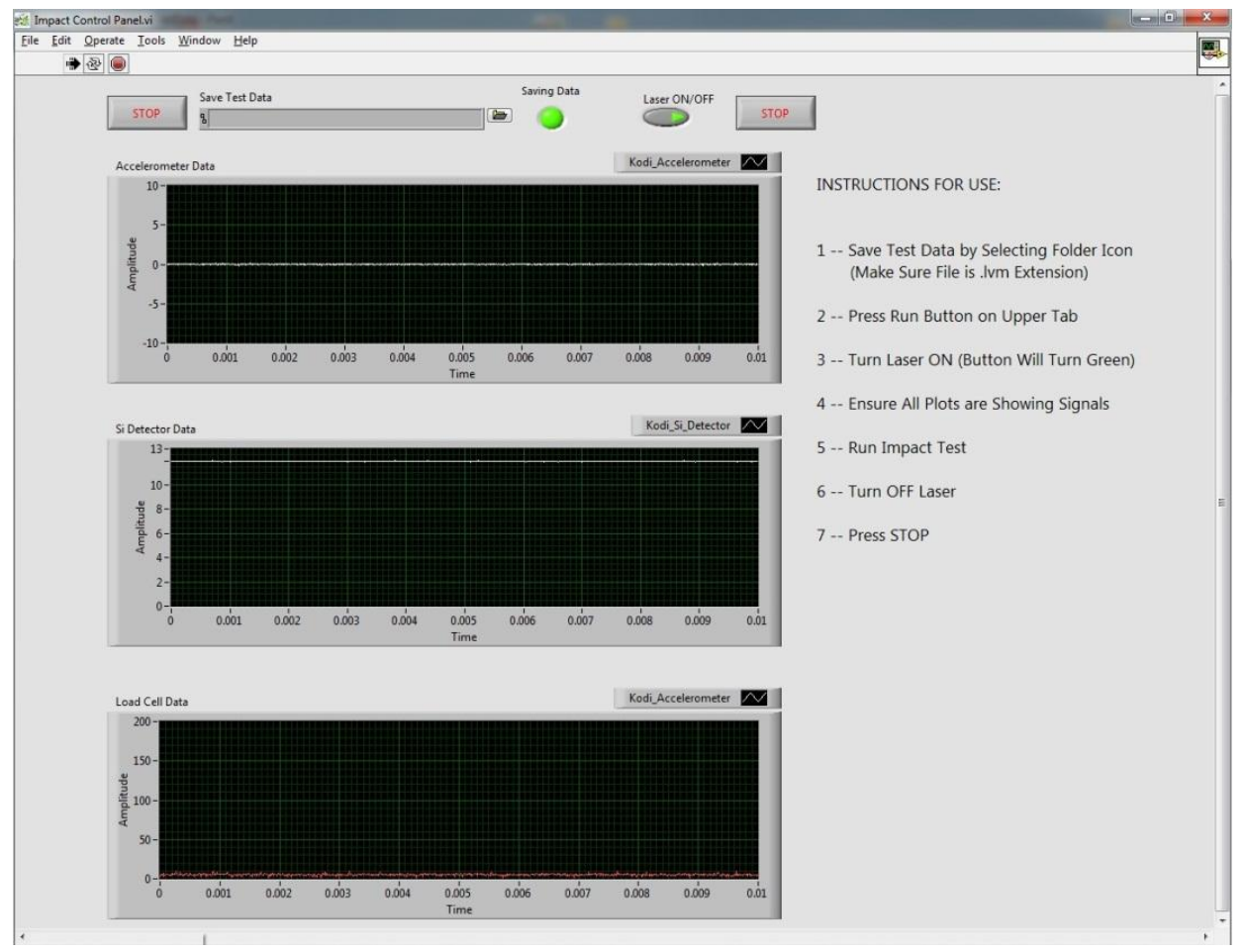

Figure 3.6. Dynatup DAQ software window with data acquisition in progress.
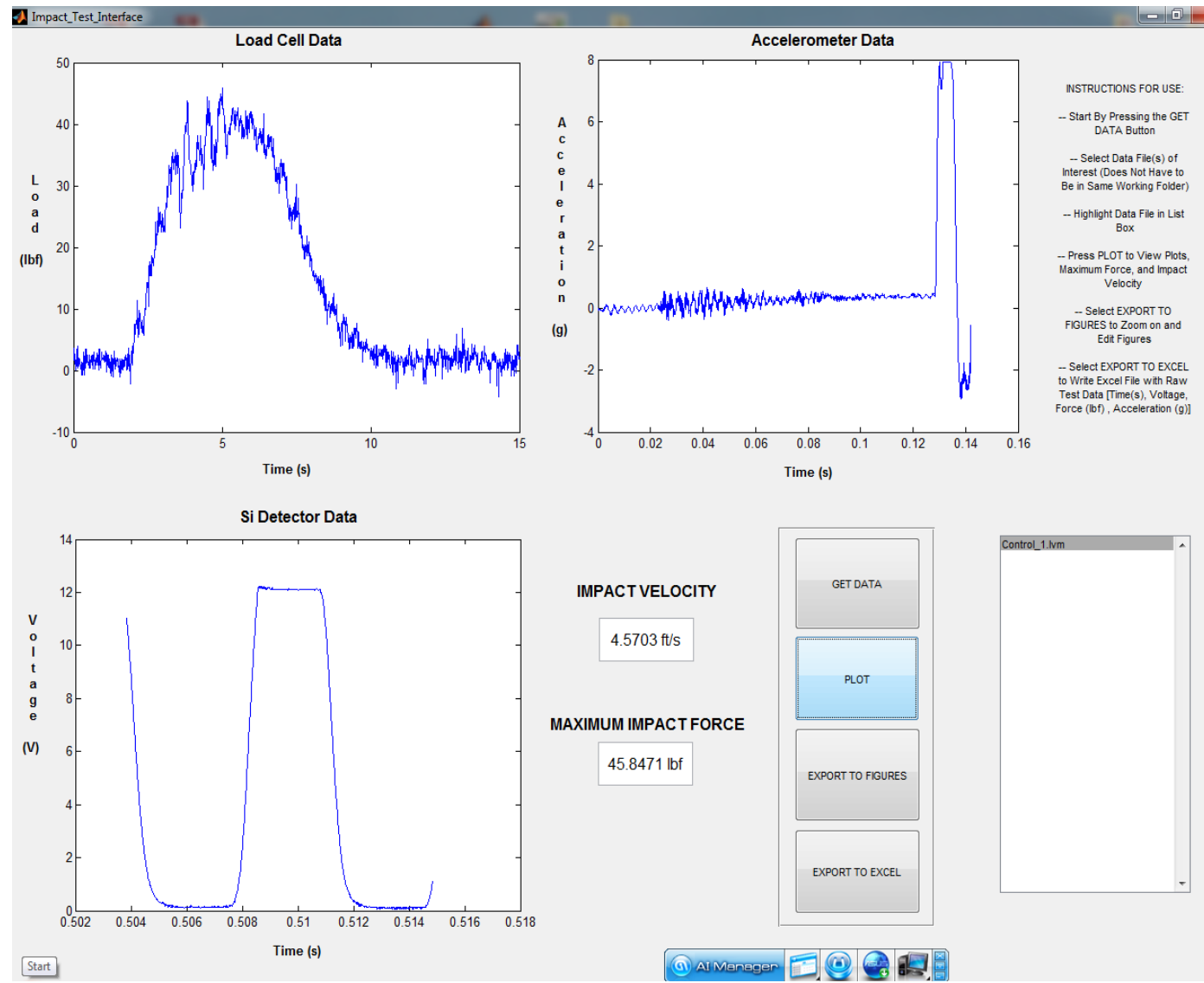
Figure 3.7. Screen shot of GUI with plots from impact test data.

Since the weight of the impactor tup was held constant, the initial resting height of the impactor tup was adjusted in order to ensure non-destructive testing. A Fowler dial depth gauge, shown in fig. 3.8, was used to measure the impact penetration as a means of validation of nondestructive testing. Several drop test heights were investigated until a final drop test height was chosen and deemed acceptable. Figure 3.9 depicts several impact penetrations to the several drop heights investigated in this research, including heights of $47,40,35,25,20$, and 15 -inches. In fig. 3.10, it can be seen that at various drop test heights, the tup did actually penetrate through the top face sheet and caused fiber breakage. The drop test height of 15 -inches showed no sign of tup penetration and the impact penetration ranged from 0.025 to 0.030 -inches, which were within the overall thickness tolerance of the beams themselves. Therefore, for all impact tests, the 7.56-lb impactor dropped from an initial resting height of 5.5-inches above the specimen, which correlated to the 15 -inch drop test height.

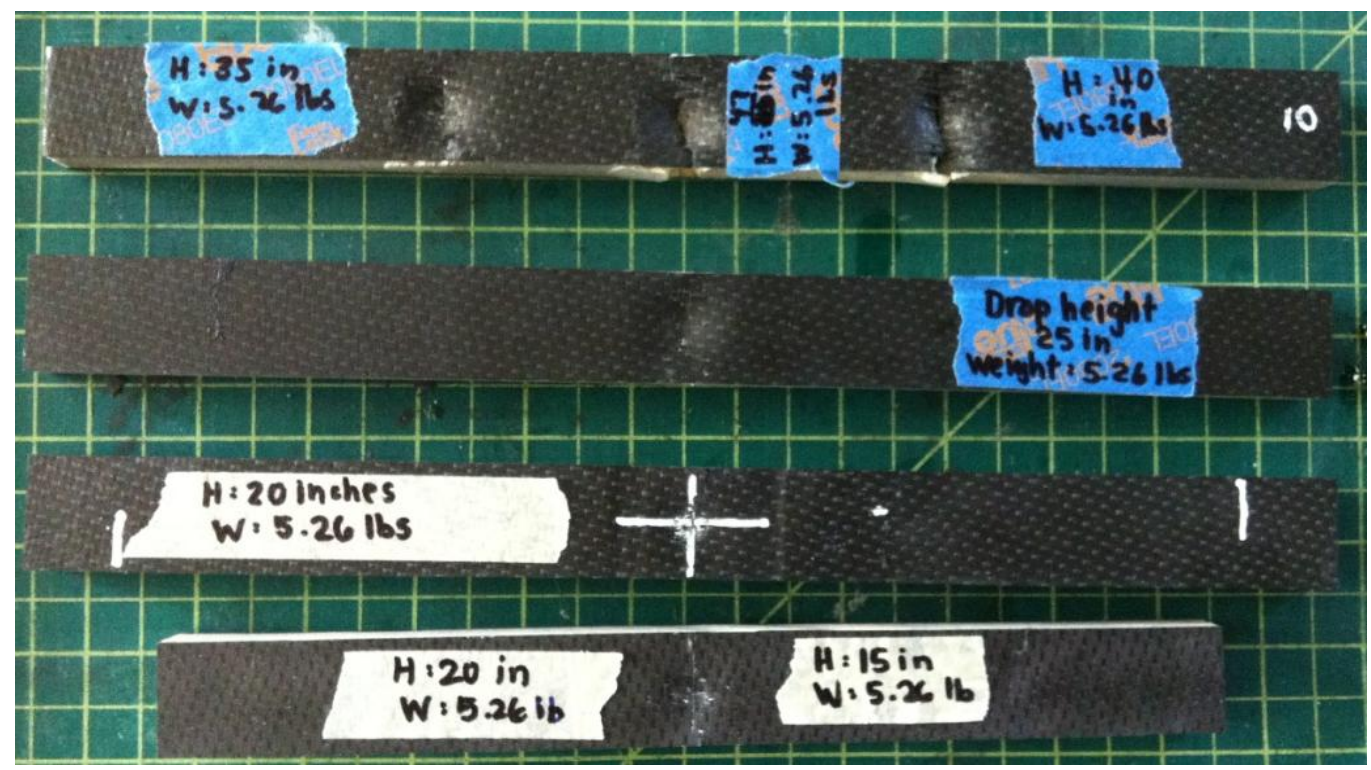

Figure 3.9. Several drop test penetrations tested to choose ideal drop weight height for all test specimens. 


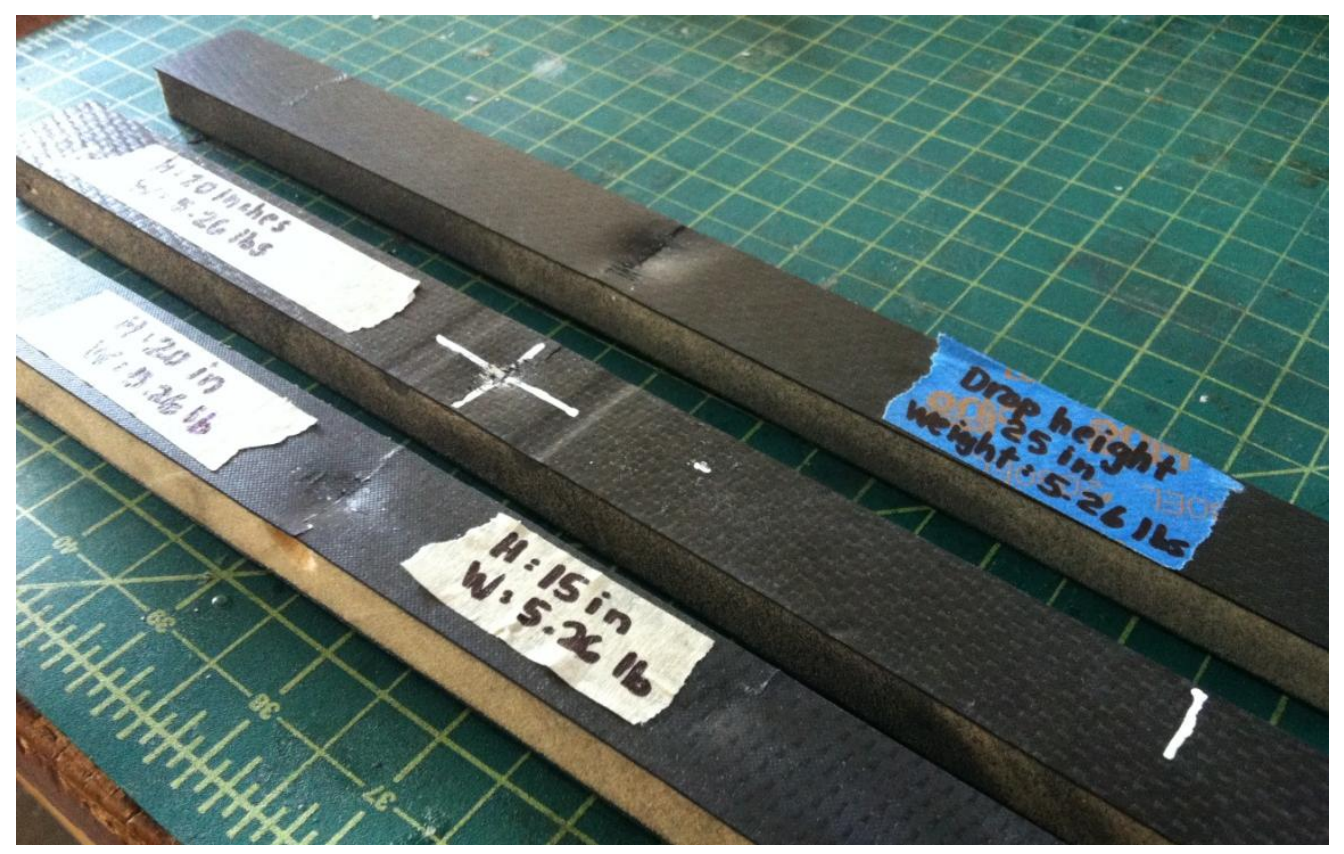

Figure 3.10. Side view of drop test penetrations.

\subsection{4-point Bend Fatigue Test}

Fatigue testing did not follow a specific ASTM standard like the other testing procedures. Therefore, the development of a custom fatigue test procedure was necessary to obtain the fatigue life of each test specimen beam investigated in this research. Fatigue testing performed in this research required the use of the Instron 8801 Servohydraulic Fatigue Testing System in the Cal Poly Aerospace Structures/Composites laboratory, shown in fig. 3.12. In this test, test specifications closely followed the static 4-point bend testing discussed in section 3.1, including the test specimen dimensions and support/load spans. However, differing from static 4-point bend testing, fatigue testing required the use of the Instron WaveMatrix software. This controlled the Instron 8801 servo-hydraulic testing system, which was run on an attached controller and data acquisition PC. The software used for fatigue testing yielded the number of cycles the test specimen beam could withstand before failure, maximum/minimum load, and maximum/minimum 
deflection. A screen shot of real time plots from the WaveMatrix software during fatigue testing is shown in fig. 3.13. For 4-point bend fatigue testing, the test specimen beams were ramped to a mean load and then subjected to a dynamic loading at constant amplitude and frequency. A diagram of this loading sequence is shown in fig. 3.11 .

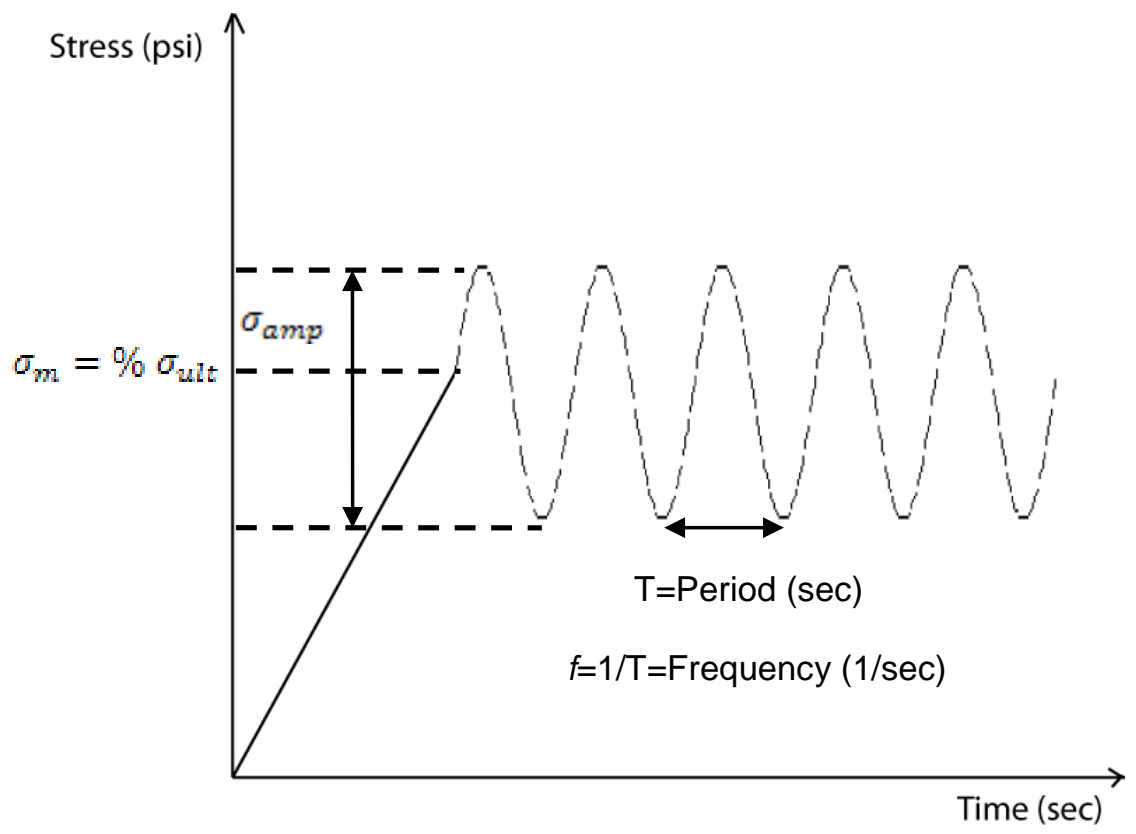

Figure 3.11. Fatigue test loading diagram.

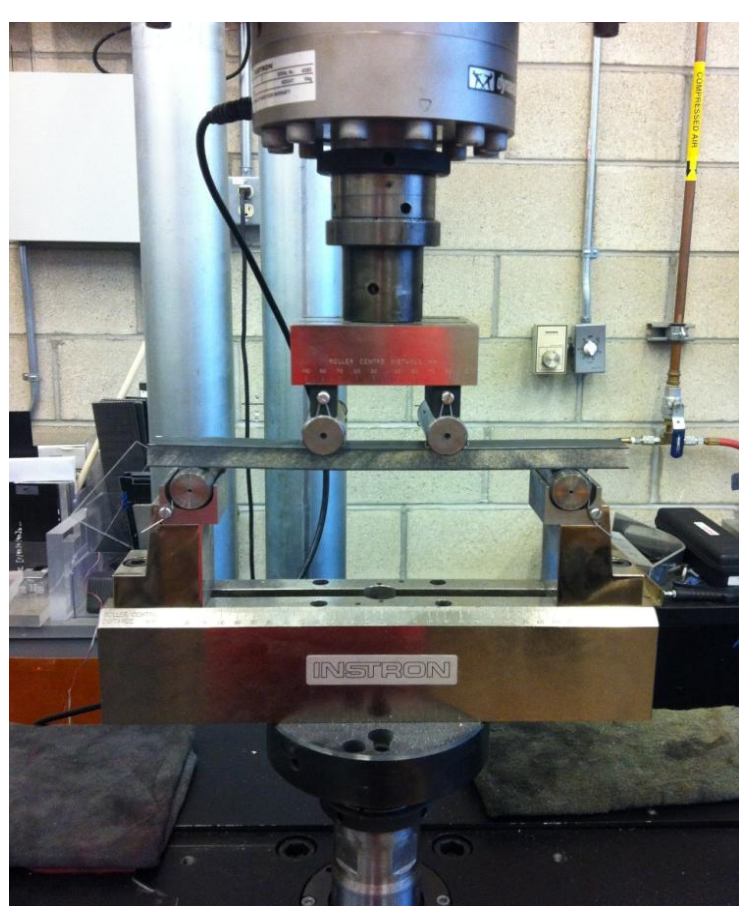

Figure 3.12. Test specimen beam secured in 4-point bend jig for 4-point bend fatigue testing. 


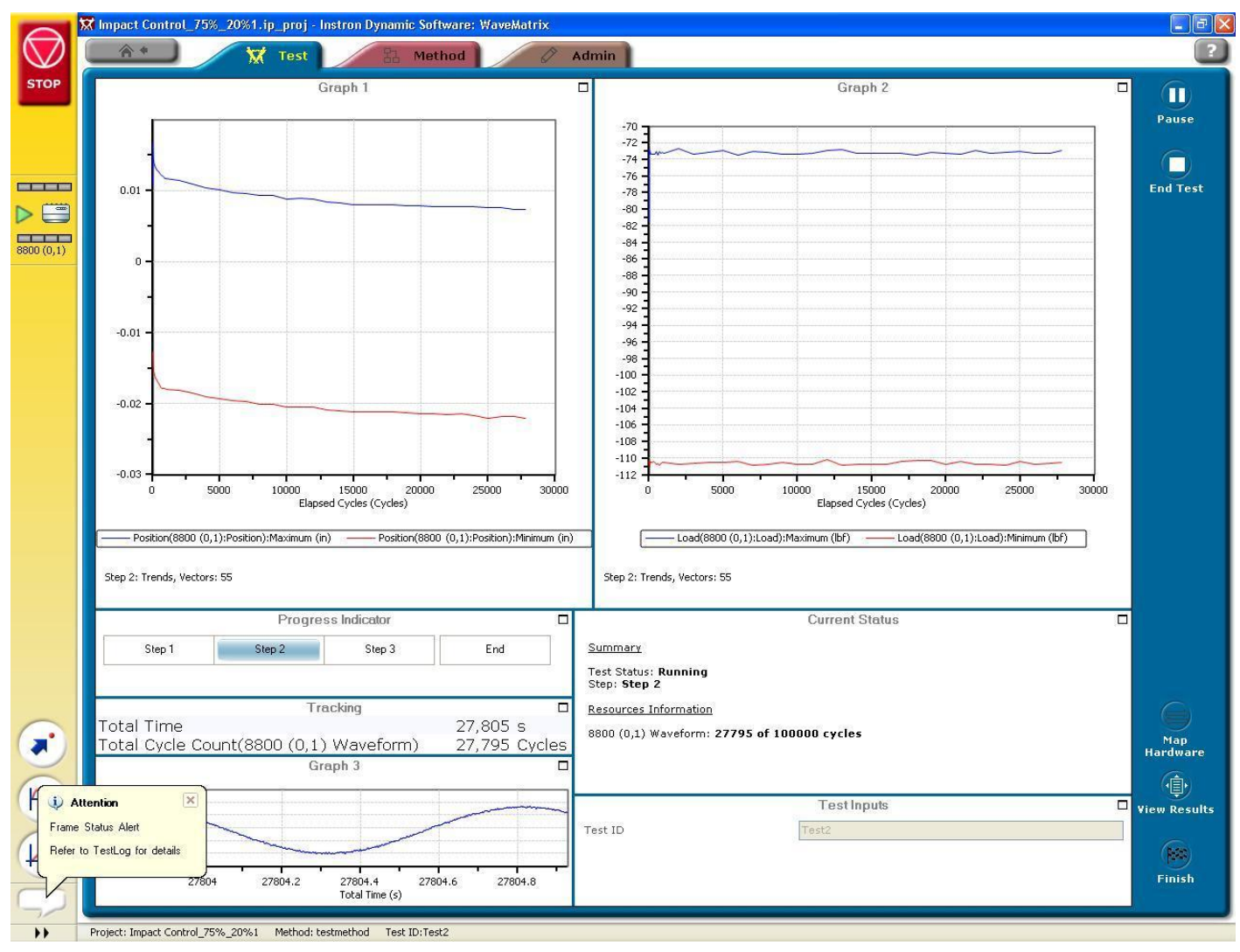

Figure 3.13. Screen shot of Wavematrix plots during a dynamic 4-point bend test.

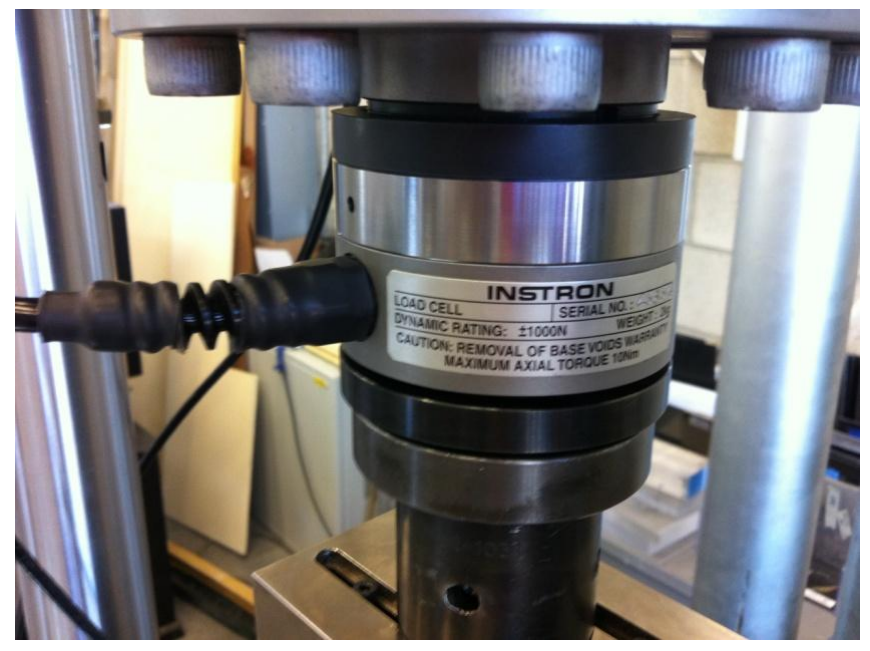

Figure 3.14. Small load cell used to perform 4-point bend fatigue testing on test specimen beams.

The development of the fatigue testing procedures was quite challenging. Testing first began with the use of a smaller load cell rated to $\pm 1 \mathrm{kN}$, shown in fig. 3.14. Changing the load cell allowed for more accurate load cell readings of the loads that were imposed on the test specimen beams, because of the small amplitude applied to the test specimens. Fatigue testing with the 
small load cell only went as far as testing one test beam case before a malfunction of the load cell occurred. Fatigue testing was performed at a frequency of $5 \mathrm{~Hz}$. This frequency was chosen because the lower the frequency, the more accurate the results are. Further, a lower frequency better models the environments to which structures are exposed to. Ideally, a frequency of $1 \mathrm{~Hz}$ yields the most accurate results but since $5 \mathrm{~Hz}$ was still a reasonable value, it was chosen as it saved time.

After the malfunction of the small load cell, the bigger load cell, rated to $\pm 100 \mathrm{kN}$, was configured for use with the WaveMatrix software. Initially, the $100 \mathrm{kN}$ load cell did not yield as precise load readings as the $1 \mathrm{kN}$ load cell, but with alterations to the control loop gains of the load cell, the results improved significantly and matched more closely with the $1 \mathrm{kN}$ load cell readings. As shown in fig. 3.15, the proportional, integral, and derivative gains were all adjustable using the loop tuning tool from the Instron software. A sinusoidal input at $1 \mathrm{~Hz}$ was used to determine the optimum proportional gain value in the loop tuning tool. In this case, the proportional gain was increased from $-17 \mathrm{~dB}$, until the sinusoidal wave of the input closely matched the sinusoidal wave of the actual load reading output. This value was determined to be $36 \mathrm{~dB}$. Since loop tuning was done at $1 \mathrm{~Hz}$, the frequency for fatigue testing was decreased from $5 \mathrm{~Hz}$ to $1 \mathrm{~Hz}$ to maintain the accuracy of the results, and thus, compensate for the small load values applied to the test specimens. Similar to the static 4-point bend testing and the fatigue testing using the $1 \mathrm{kN}$ load cell, the test specimens were loaded in the 4-point bend jig, where the beam was centrally located between the supports. Instron WaveMatrix software was used to control the Instron 8801 servo-hydraulic testing system, which is run on an attached controller and data acquisition PC. The software used for fatigue testing yielded the number of cycles the test specimen beam could withstand before failure, maximum/minimum load, and maximum/minimum deflection. The control test specimen beams were ramped to a mean load and then subject to dynamic 4-point bend flexural loading at constant amplitude and frequency. 


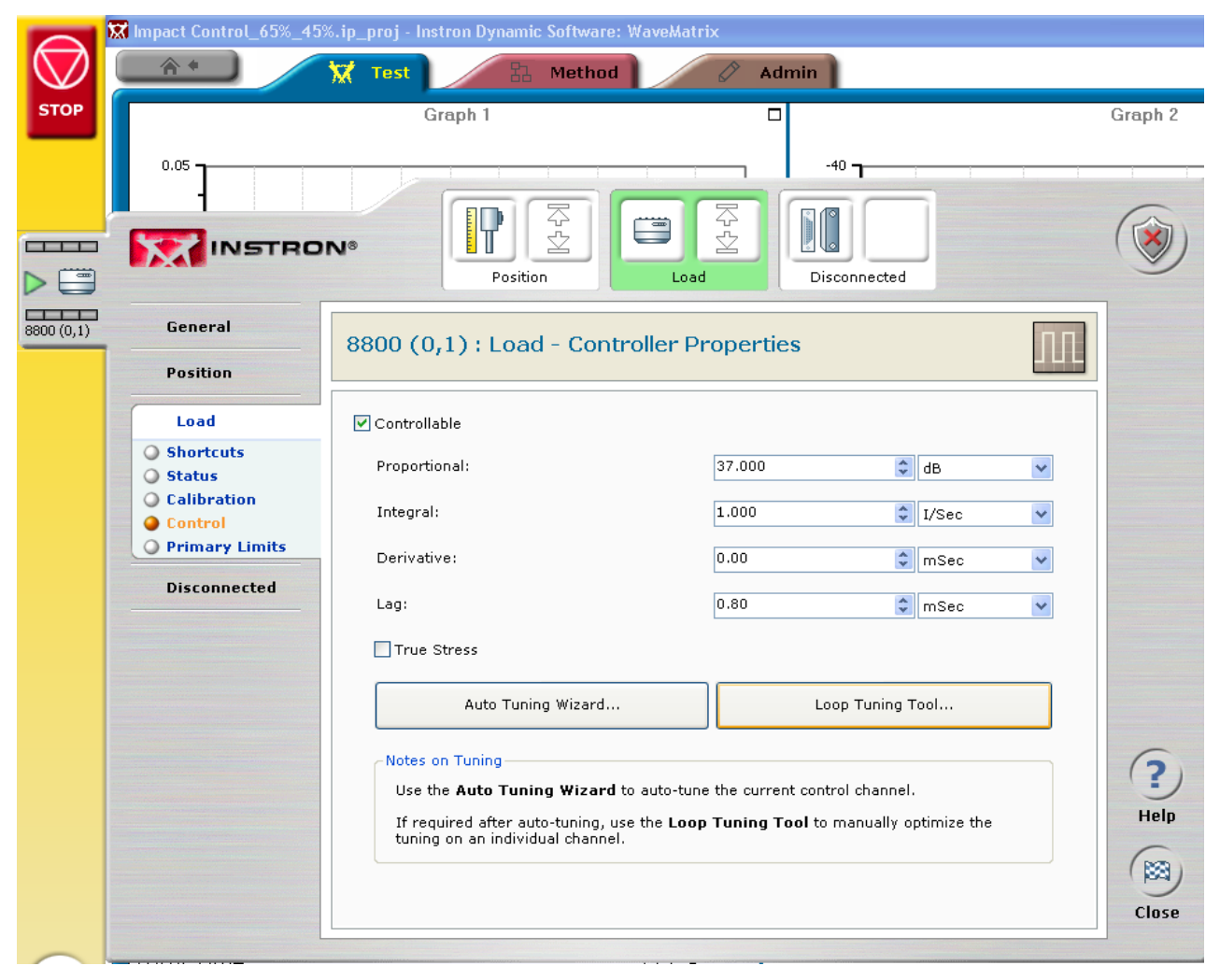

Figure 3.15. Control loop tuning tool for the Instron. 


\section{Chapter 4 Experimental Test Results}

This section presents the results of the experimental tests of static 4-point bending of test specimen beams, impact of the test specimen beams, and 4-point bend fatigue testing of test specimen beams. Ultimate load, strain in the outer fibers, maximum stress, and the bending modulus of elasticity were determined for each test specimen beam case investigated under static 4-point bending. Stress versus strain plots of the test specimen beam cases under static 4point bending are presented as well. For each case under static 4-point bend, failure modes were determined and are discussed in detail. Impact test results included the average maximum impact force experienced by the test specimen and the average impact velocity. Further, impact penetrations and depth of the penetrations were also examined and are presented. Fatigue life cycles of each test beam case are presented for the 4-point bend fatigue testing and failure modes of each case are presented and discussed in detail, including pre-impact and post-impact tests.

\subsection{Static 4-Point Bend Test}

\subsubsection{Control Beam}

Eight control test specimens, manufactured using the autoclave method discussed in section 2.1, were tested according to test standard ASTM D6272 discussed in section 3.1. Time, cross-head position, load, deflection, and strain were obtained using Bluehill 2 software. Of these test parameters, only the load and deflection values were used to determine important values such as ultimate strength. The load versus deflection data was plotted for each non-delaminated control sample as seen in fig. 4.1. 


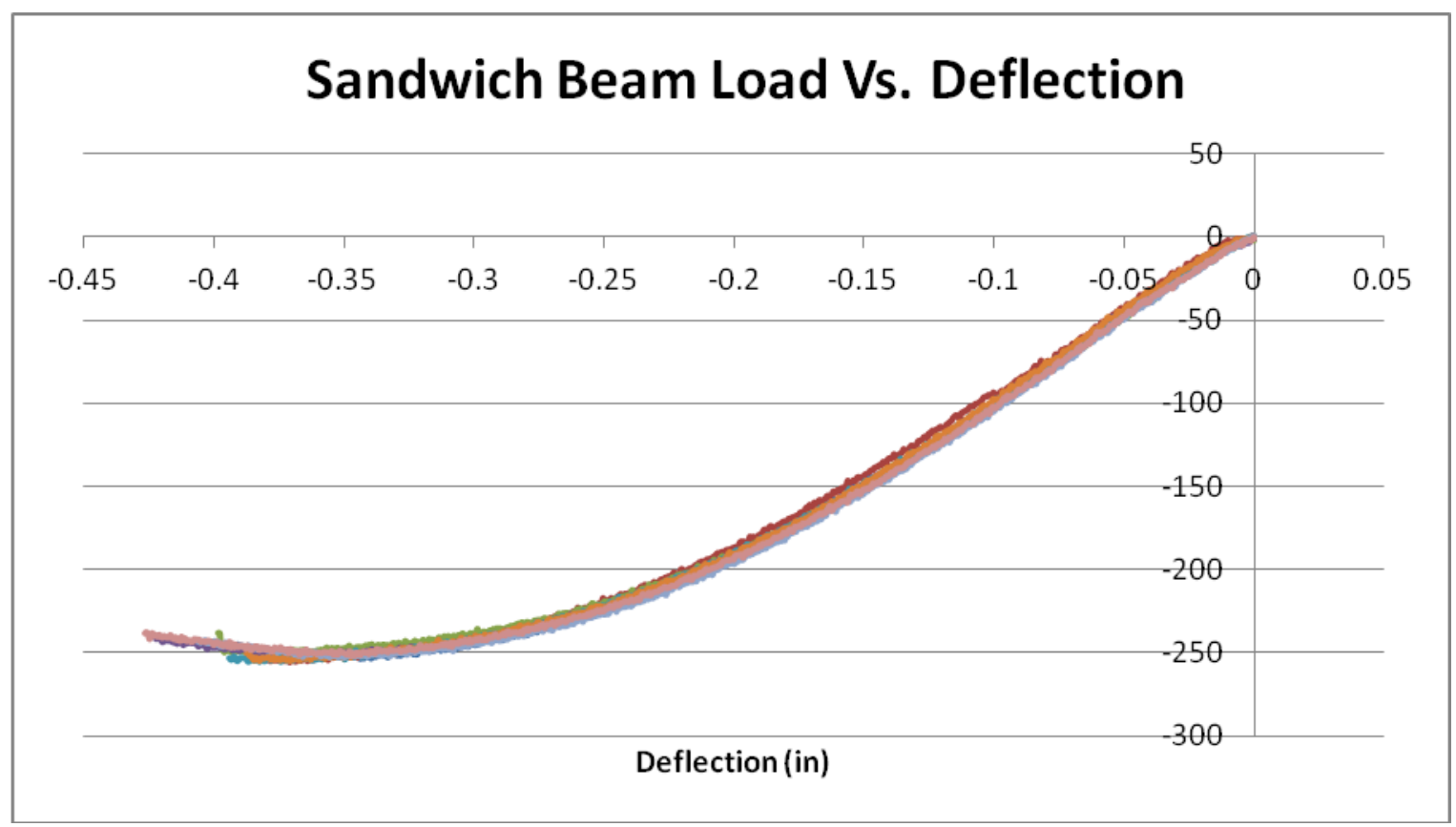

Figure 4.1. Sandwich beam load vs. deflection used to find mechanical properties.

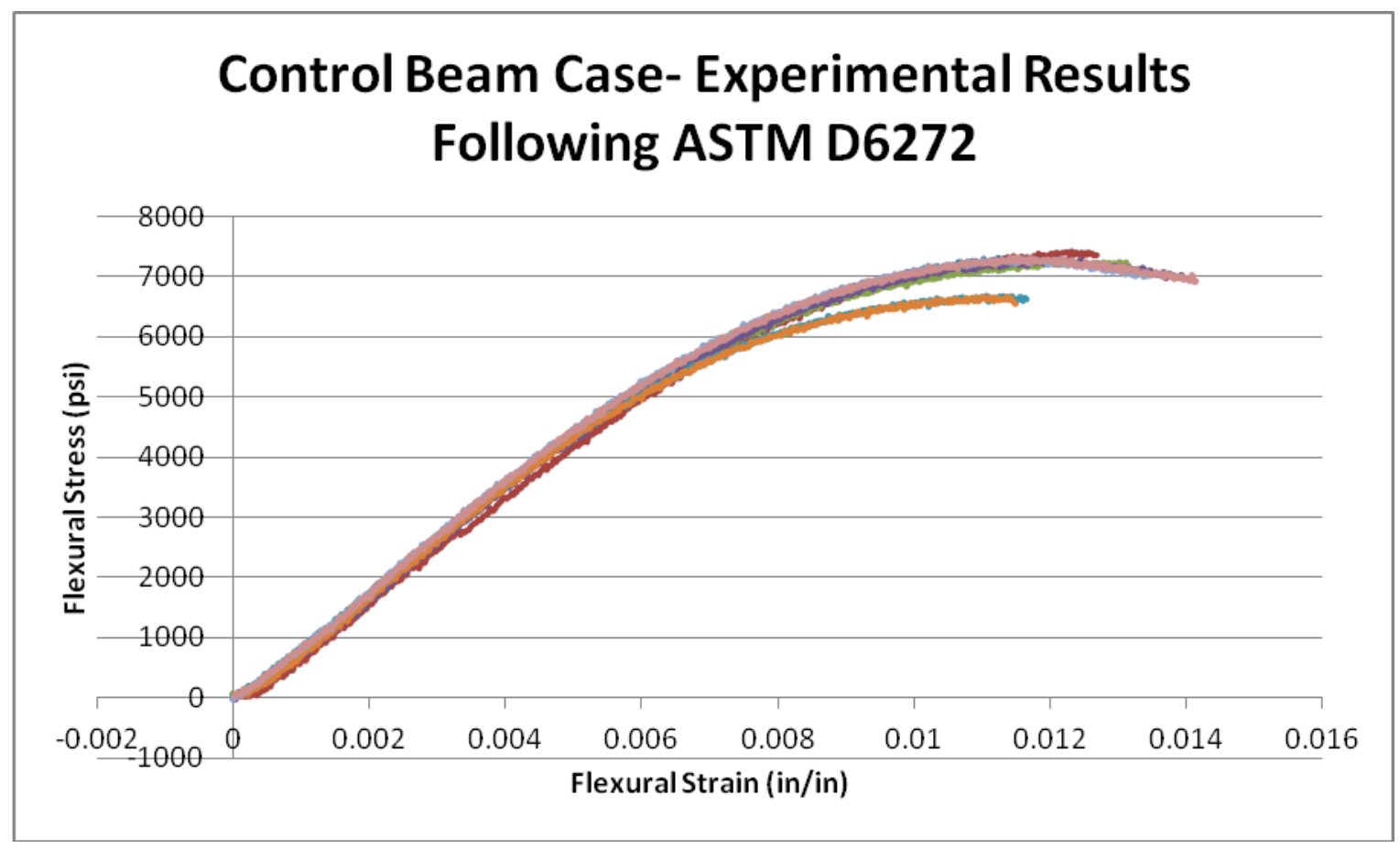

Figure 4.2. Resulting flexural stress-strain curves of the control case beam specimens under 4-point bending.

Flexural parameters were calculated after obtaining the experimental curves in order to have a quantitative means of comparing the performance of each test case. Quantitative performance parameters are given in the ASTM testing standard and include: maximum stress in 
the outer fiber throughout the load span, maximum strain in the outer fibers, and modulus of elasticity in bending [27]. Maximum stress was determined utilizing eqn. 4.1:

$$
\sigma_{\max , f}=\frac{P_{\max L}}{b d^{2}}
$$

where $\mathrm{P}_{\max }$ is the maximum applied compressive load prior to failure. Maximum strain was determined utilizing eqn. 4.2:

$$
\varepsilon_{\max , f}=4.70 \frac{D d}{L^{2}}
$$

where $D$ is the maximum deflection prior to failure and 4.70 is a correction factor indicating that the specimen was tested under a load span that was one-third the total support span. In addition the elastic modulus of the test specimen in bending was determined utilizing eqn. 4.3:

$$
E_{B}=0.21 \frac{L^{3} m}{b d^{3}}
$$

where $\mathrm{m}$ is the slope of the tangent to the initial straight line of the test specimen load vs. displacement curve and 0.21 is, again, a correction factor indicating that the specimen was tested under a load span with one-third the total load span.

The average elastic modulus of the eight test beams was found to be $0.84-M s i$ with a standard deviation of $\pm 12 \%$. The ultimate strength of the sandwich beam is equal to the maximum stress in the outer fibers at the moment the beam breaks. The average ultimate strength of the sandwich beams was 7,364 -psi with a standard deviation of $\pm 2 \%$, which is illustrated in fig. 4.2 , where stress and strain data are plotted. The failure mode observed for each test specimen in the control case group was very similar for each, as depicted in fig. 4.4. 


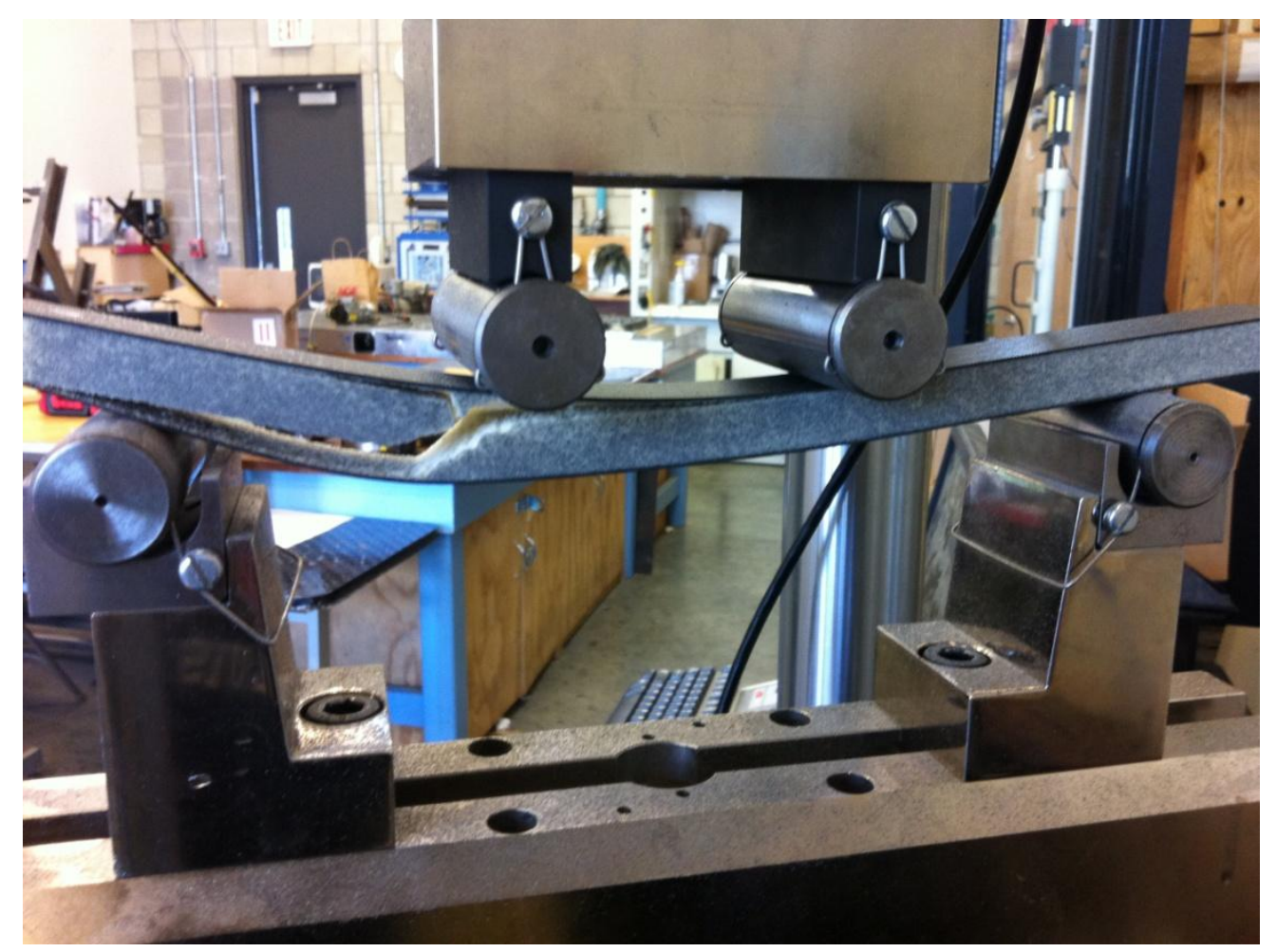

Figure 4.3. Typical failure mode of control case beam subjected to static 4-point bending.

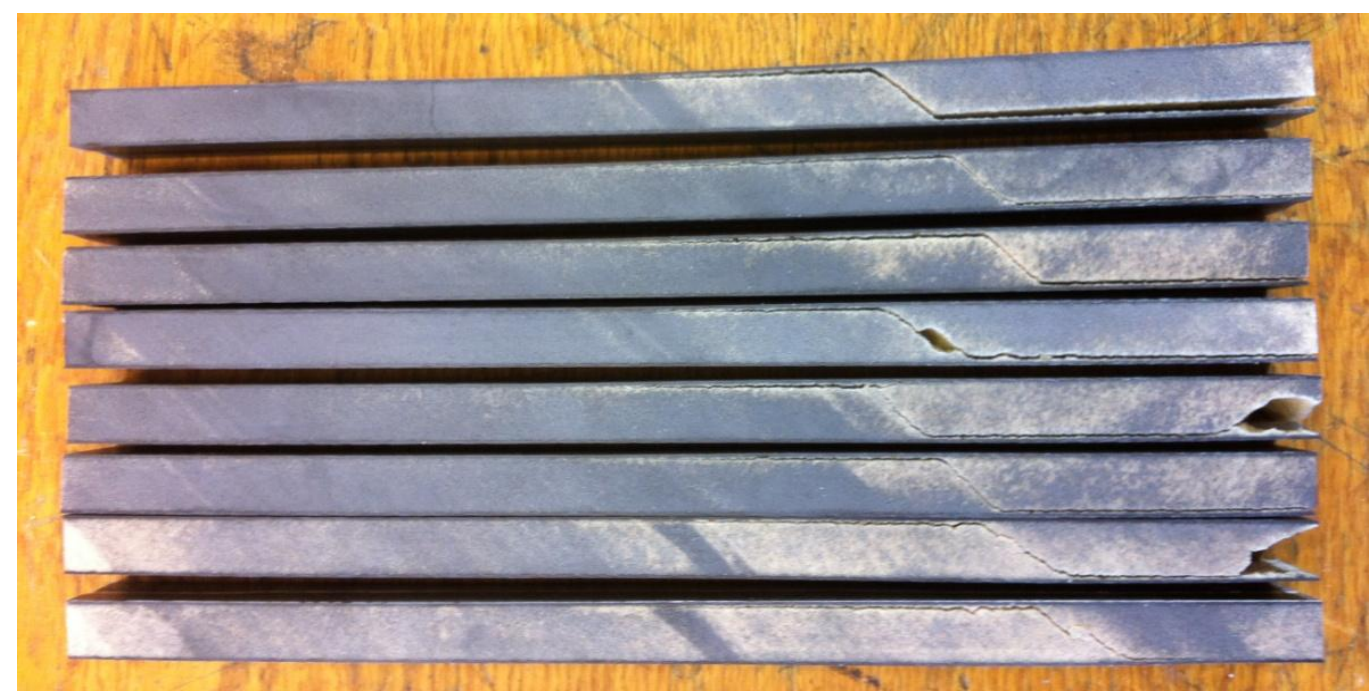

Figure 4.4. Control case group failure mode nearly identical in each test specimen.

Face core delamination was the cause of failure seen in the control beam case. Delamination initiated on the top face sheet over the right loaded cylinder and continued for about one inch until the core fractured completely through the depth of the beam and eventually reached the bottom face sheet, shown in fig. 4.3 (picture taken from back side of four-point bend fixture). This fracture caused a delamination of the core and bottom face sheet near the support, 
which continued to the right end of the beam. This failure sequence occurred in all specimens in the control test group and could be caused by a slightly imbalanced test fixture. Since there was no evidence of face sheet failure, it can be noted that the flexural strength of the control test specimen was dependent on the actual strength of the foam core and the shearing strength of the bond between the face and core materials.

\subsubsection{Beam with Transverse DADs}

Six test specimens of the transverse DAD key case with no initial delamination were tested to ultimate failure. An average ultimate load of 210.89 -lb with a standard deviation of $\pm 4 \%$ was determined for the test specimens subjected to static 4-point bending.

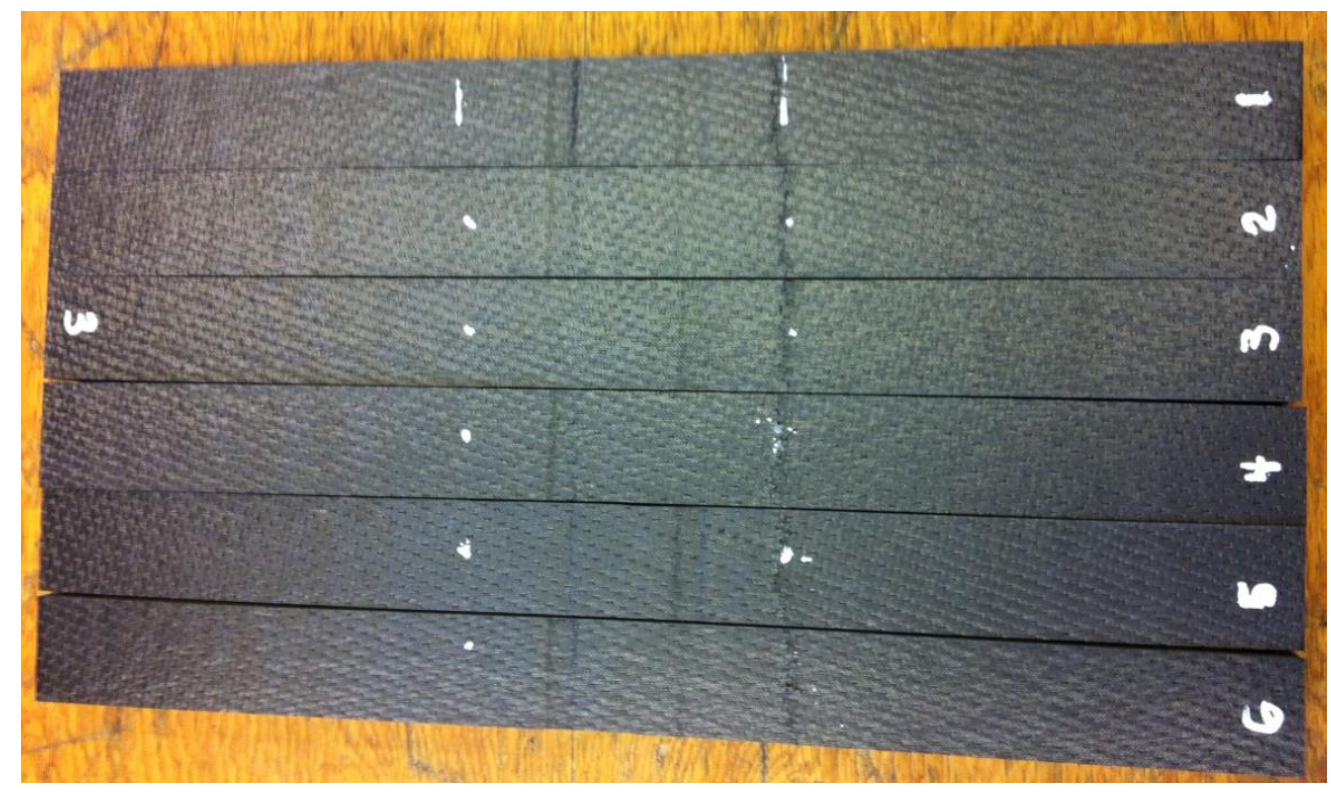

Figure 4.5. Final failure mode of composite sandwich beam test group with added transverse DAD keys.

The failure mode was unique to the previously tested case in that face-core delamination was not the initial failure mode. As shown in fig. 4.5, all specimens in the test group ultimately failed due to face sheet fracture. One can see from the figure that face sheet fracture occurs near the right DAD key location, and under loading this fracture occurred directly under the right loaded cylinder. Once testing was completed for all six test specimens, resulting flexural stress vs. strain curves were obtained from the testing software for each specimen, and are shown in fig. 4.6. The ultimate strength of all samples was found to be 6134 -psi with a deviation of $\pm 4 \%$ error. The ultimate strength value found through experimentation shows that there is a $17 \%$ 
decrease in strength over the control beam case without transverse DAD keys. In this case, the DAD keys did prevent the occurrence of delamination in each beam. However, ultimate strength of the test specimen was compromised. This can be due to the fact that adding the fiberglass inserts increased the stiffness of the system as a whole and under a 4-point bend static test, can be detrimental to the system.

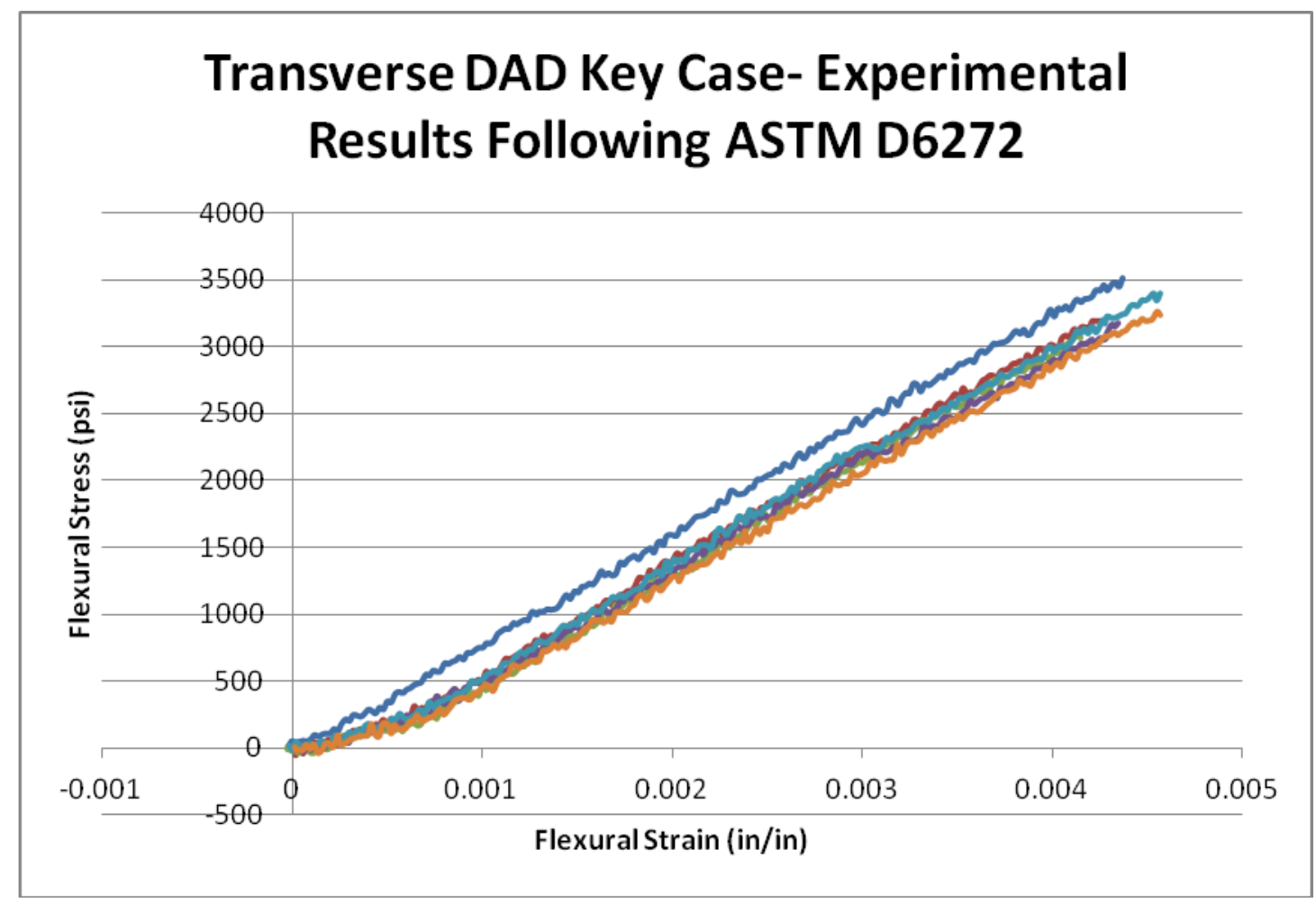

Figure 4.6. Resulting flexural stress-strain curves of specimens with no initial delamination and added transverse DAD keys subjected to static 4-point bending.

\subsubsection{Beam with Longitudinal DAD}

Eight test specimens of the longitudinal DAD key case with no initial delamination were tested to ultimate failure. An average ultimate load of 204.40 -lb with a standard deviation of $\pm 2 \%$ was determined for the test specimens subjected to static 4-point bending. 


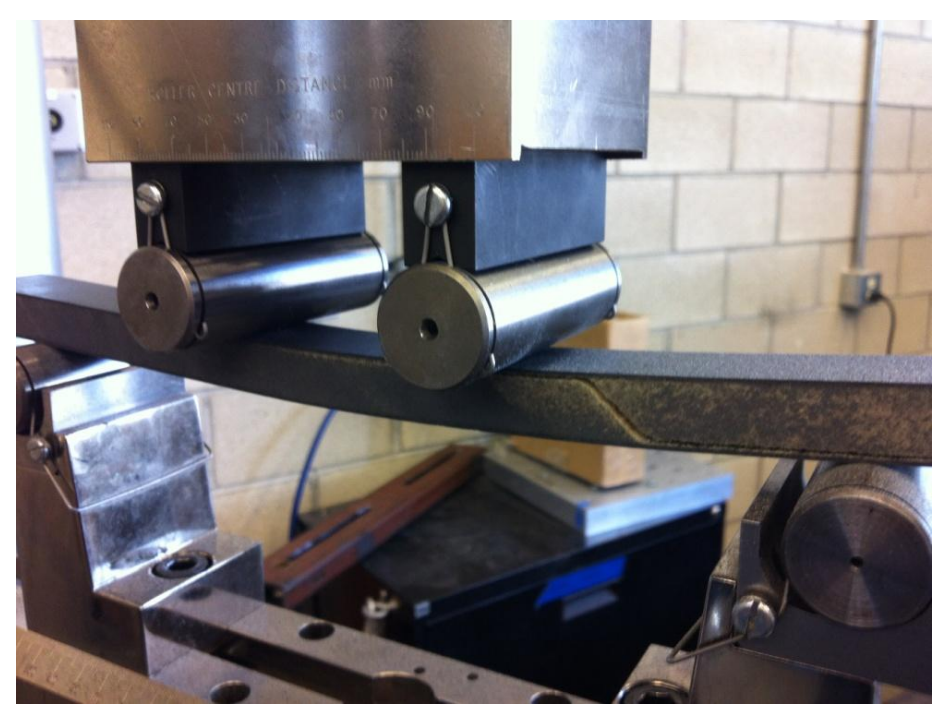

Figure 4.7. Final failure mode of composite sandwich beam test group with added longitudinal DAD keys and no initial delamination.

The failure mode was similar to most of the previously tested cases in that face-core delamination was the initial failure mode. As shown in fig. 4.7, all specimens in the test group ultimately failed similarly to the control beam case with no initial delamination or added DAD keys. One can see from the figure that face sheet delamination begins near the left DAD key location, and propagates for about four inches until core fracture occurs. This shows that the DAD key does actually delay the occurrence of core fracture, but still fails prematurely. Once testing was completed for all eight test specimens, resulting flexural stress vs. strain curves were obtained from the testing software for each specimen, and are shown in fig. 4.8. The ultimate strength of all samples was found to be 5946-psi with a deviation of $\pm 2 \%$ error. The ultimate strength value found through experiment shows that there is a $19 \%$ decrease in strength over the control beam case without DAD keys and no initial delamination. In this case, the DAD keys did not prevent the occurrence of delamination, as the transverse DAD keys did. However, ultimate strength of the test specimen was compromised similarly to the transverse DAD key case. Since the longitudinal DAD key case covered a larger area between the face and core materials, this did add more stiffness to the system, and thus reduced the ultimate strength by $2 \%$ more than the transverse DAD key case. Adding stiffness to a system does not always affect the system negatively, but under a four 4-point static test, the addition of stiffness caused early failure to the system. 


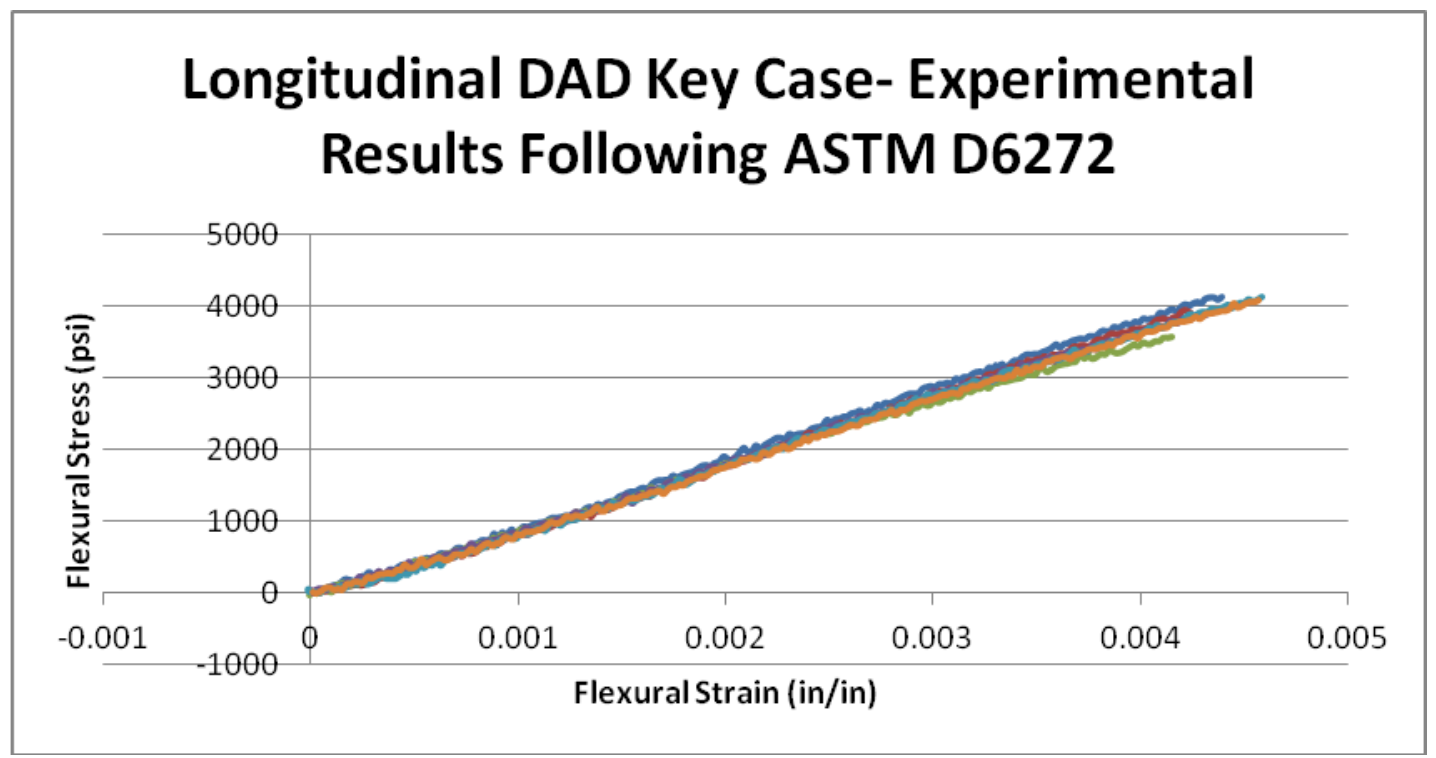

Figure 4.8. Resulting flexural stress-strain curves of composite sandwich specimens with no initial delamination and added longitudinal DAD keys subjected to static 4-point bending.

\subsubsection{Initially Delaminated Beam}

In order to understand the effects of delamination on a composite sandwich, it was pertinent to introduce a form of delamination in the control beam. A one inch delamination was forced as discussed in section 2.4. Again, eight test specimens were chosen to be tested under 4-point bend. For this case, it was imperative that the delamination was placed directly in between the load span to yield consistent results. As shown in fig. 4.9, initial failure occurred due to face-core delamination in between the load span in all test cases. An average ultimate flexural load of 102.58 -lb with a standard deviation of $\pm 6 \%$ error was determined.

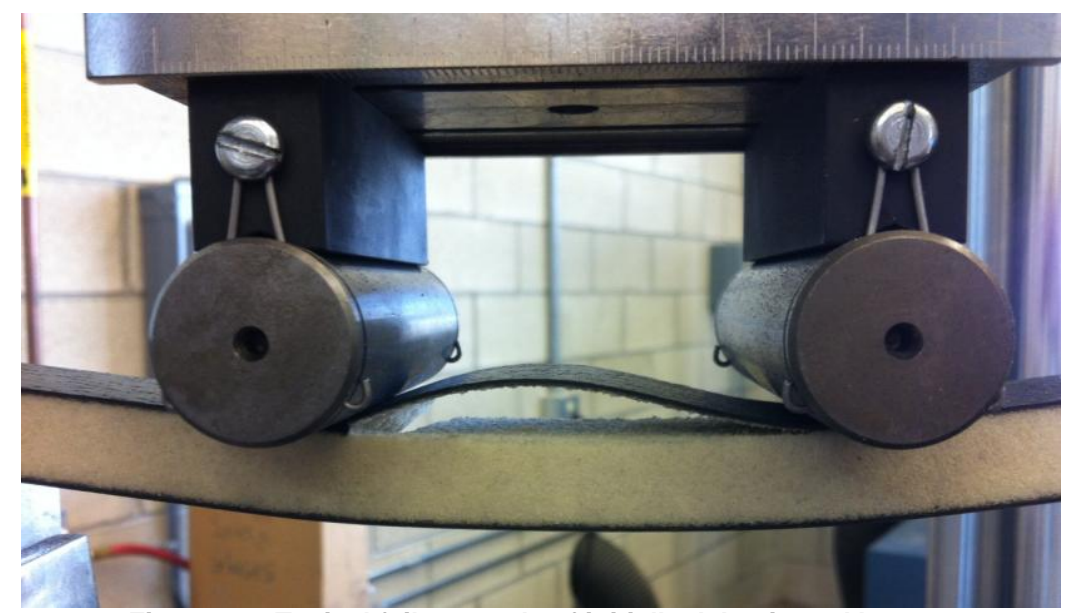

Figure 4.9. Typical failure mode of initially delaminated beams. 


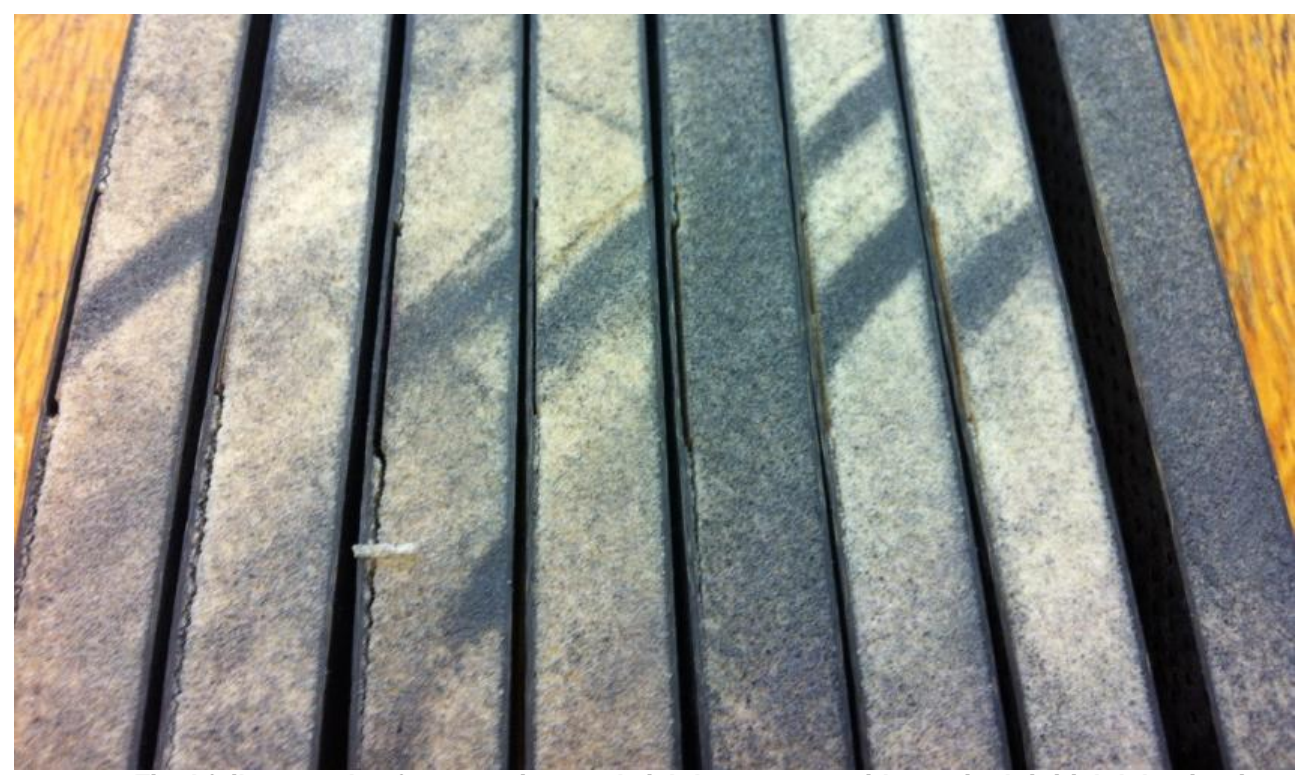

Figure 4.10. Final failure mode of composite sandwich beam case with one inch initial delamination.

The initially delaminated beam case initially failed similarly to the control case, where there was an evident face-core separation under the load span area that continued one inch past the left loaded cylinder. However, the initially delaminated case beam differed in the final failure, where the delamination also continued past the right loaded cylinder. The delamination in this case is symmetrical and continues about one inch past the initial delamination in both directions, as seen in fig. 4.10. In this case, there is no complete failure through the core to the other face sheet, as seen in the control case. This resulted in a $60 \%$ decrease in flexural strength of the beams with an initial one inch delamination compared to the control case. Again, no visible failure to the composite face sheet was observed, which indicates that the flexural strength of the composite sandwich beams with an initial one inch delamination was largely driven by the flexural strength of the foam core. After obtaining displacement and load parameters from the testing software, stress versus strain curves of the eight test specimens were obtained. The resulting flexural stress versus strain curves are shown below in fig. 4.11 for the composite sandwich beams with an initial one inch delamination subjected to a static 4-point bend. The ultimate strength of all samples was found to be 2984 -psi with a standard deviation of $\pm 6 \%$ error. 


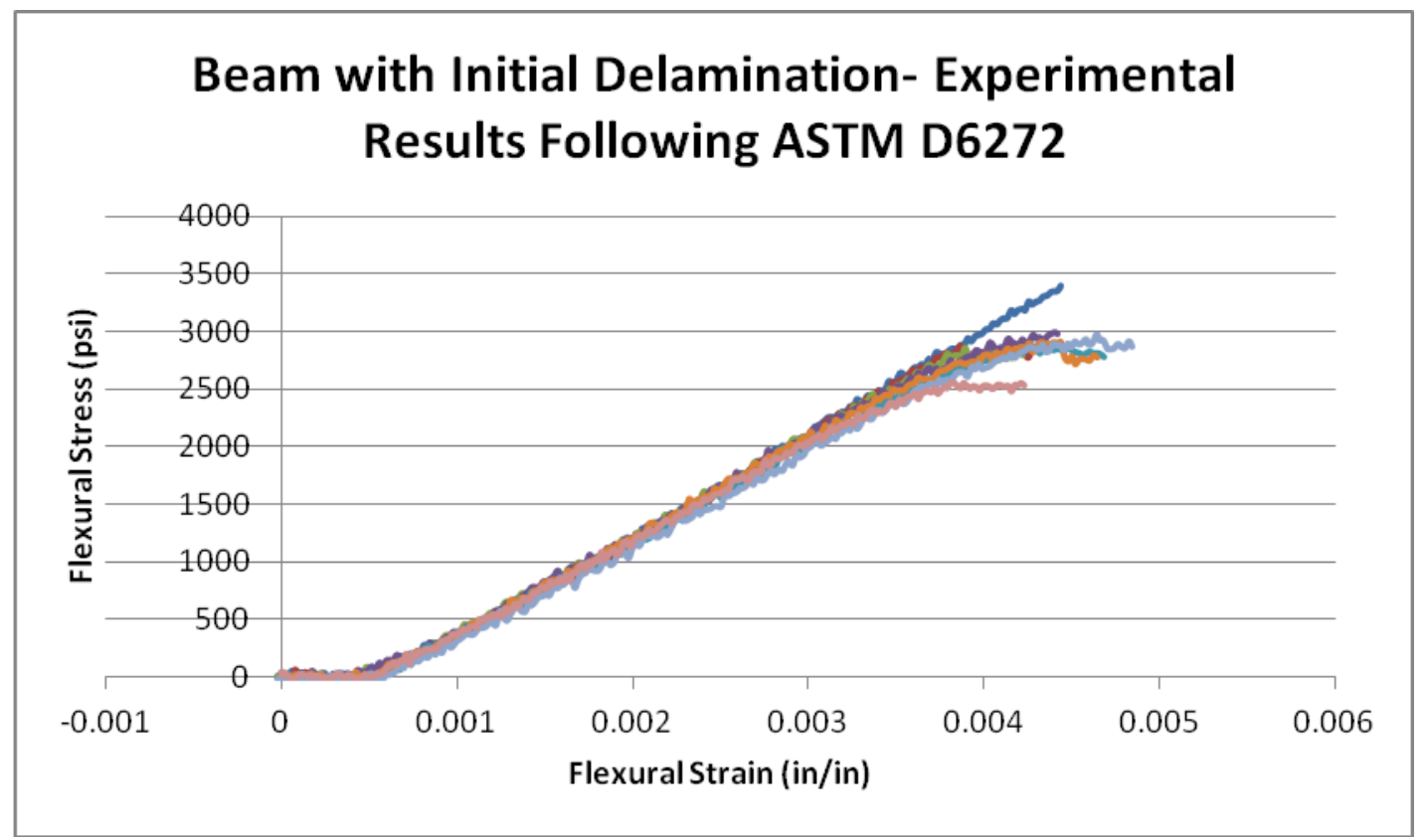

Figure 4.11. Resulting flexural stress-strain curves of test specimens with an initial one inch long delamination subjected to 4-point bending.

\subsubsection{Initially Delaminated Beam with Transverse DADs}

Five test specimens of the DAD key case with a one-inch centrally located initial delamination between the DADs were tested to ultimate failure. An average ultimate load of 220.44-lb with a standard deviation of $\pm 4 \%$ was determined for the test specimens subjected to static 4-point bending.

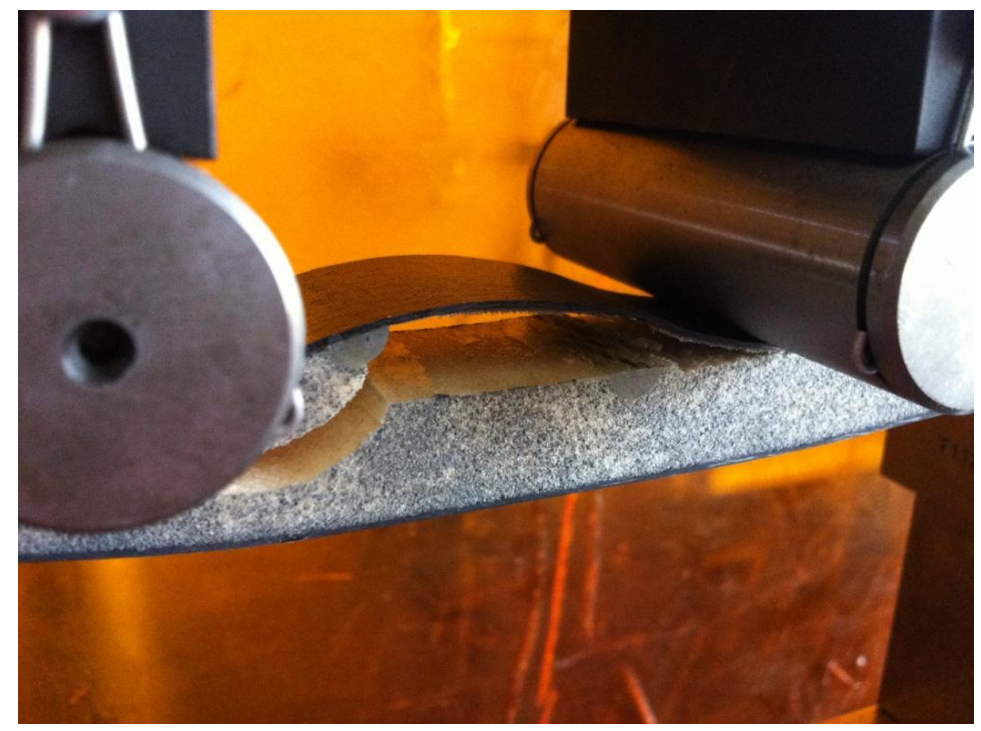

Figure 4.12. Final failure mode of composite sandwich beam with one inch initial delamination and added transverse DAD keys. 


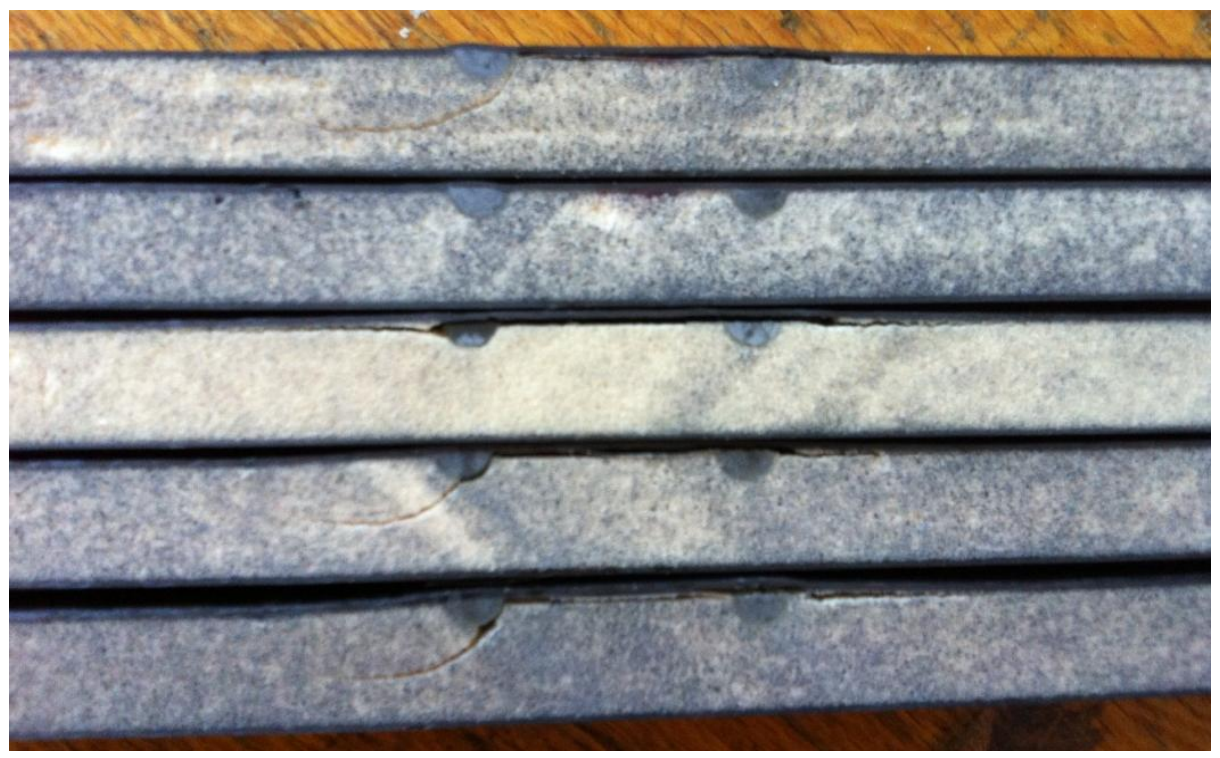

Figure 4.13. Final failure mode of composite sandwich beam test group with one inch initial delamination and added transverse DAD keys.

The failure mode was similar to the two previously tested cases in that face-core delamination was the initial failure mode. As shown in fig. 4.12, the core fracture propagated from the initial delamination towards the left loaded roller, but face-core delamination occurred at the right roller support as well. Figure 4.13 shows the observed failure mode for the transverse DAD key case beams with an initial 1 -inch long centrally located delamination. One can see that core fracture occurs near the left DAD key location with face core delamination also occurring past the area under the right roller. Once testing was completed for all five test specimens, resulting flexural stress vs. strain curves were obtained from the testing software for each specimen, and are shown in fig. 4.14. The ultimate strength of all samples was found to be 6413-psi with a standard deviation of $\pm 4 \%$ error. The ultimate strength value found through experimentation shows that there is a $115 \%$ increase in strength over the delaminated beam case without DAD keys. Although the DAD key did not eliminate the occurrence of delamination propagation, it did allow for an increase in the ultimate load of the beam. 


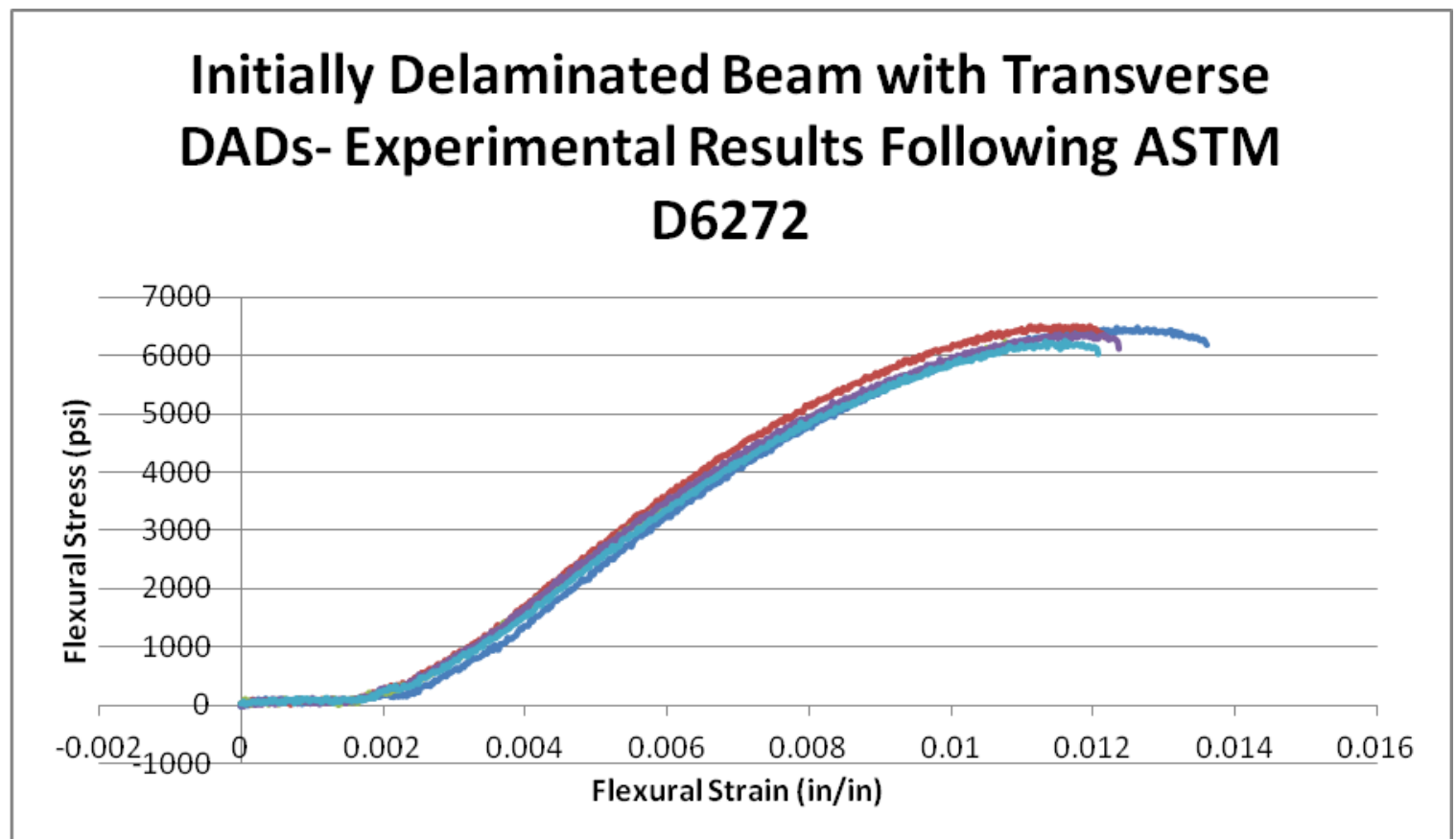

Figure 4.14. Resulting flexural stress-strain curves of composite sandwich beams with an initial one inch long delamination and transverse DAD keys subjected to static 4-point bending.

\subsubsection{Initially Delaminated Beam with Longitudinal DADs}

Five test specimens of the longitudinal DAD key case with a one-inch centrally located initial delamination and a DAD key that ran the entire length of the beam were tested to ultimate failure. An average ultimate load of 154.36 - $\mathrm{lb}$ with a standard deviation of $\pm 5 \%$ was determined for the test specimens subjected to static 4-point bending.

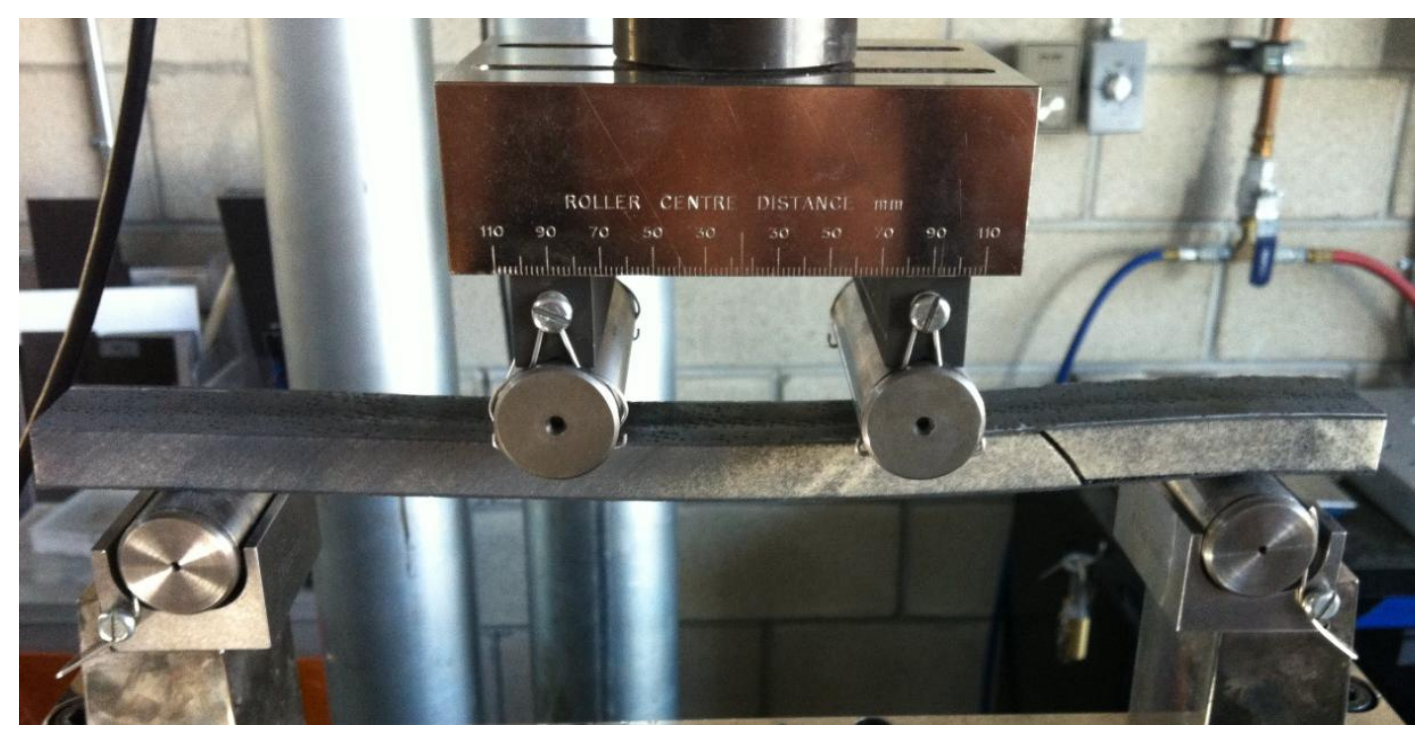

Figure 4.15. Final failure mode of composite sandwich beam with one inch initial delamination and added longitudinal DAD key. 


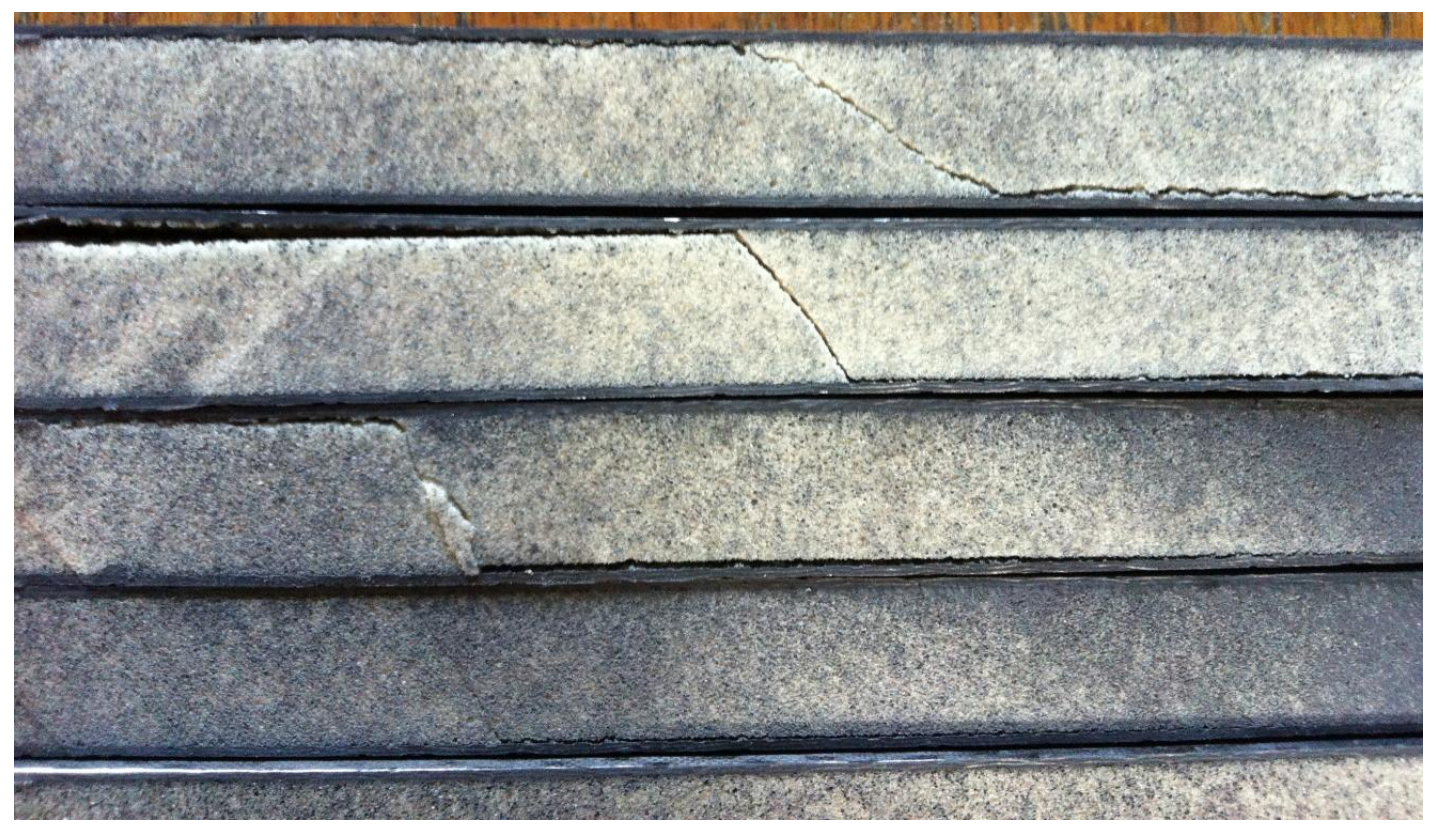

Figure 4.16. Final failure mode of composite sandwich beam test group with one inch initial delamination and added longitudinal DAD key.

The failure mode was similar to the previously tested delaminated cases such that facecore delamination was the initial failure mode. As shown in fig. 4.15, the initial delamination propagated for about 3 inches towards the right loaded roller and core fracture occurred past the right loaded roller. This shows that the longitudinal DAD key does actually delay the occurrence of core fracture, and prolongs the failure of the test beam. Figure 4.16 shows the observed failure mode for the longitudinal DAD key case beams with an initial 1-inch long centrally located delamination. One can see from the figure that core fracture occurred about 3 inches past the initial delamination and face core delamination also occurred past the area under the right roller load. Once testing was completed for all five test specimens, resulting flexural stress vs. strain curves were obtained from the testing software for each specimen, and are shown in fig. 4.17. The ultimate strength of all samples was found to be 4491 -psi with a standard deviation of $\pm 5 \%$ error. The ultimate strength value found through experiment shows that there is a $66 \%$ increase in strength over the delaminated beam case without DAD keys. Similar to the transverse DAD key case, the longitudinal DAD key did not eliminate the occurrence of delamination propagation. However, adding the longitudinal key to the delaminated beam did allow for an increase in the ultimate load. 


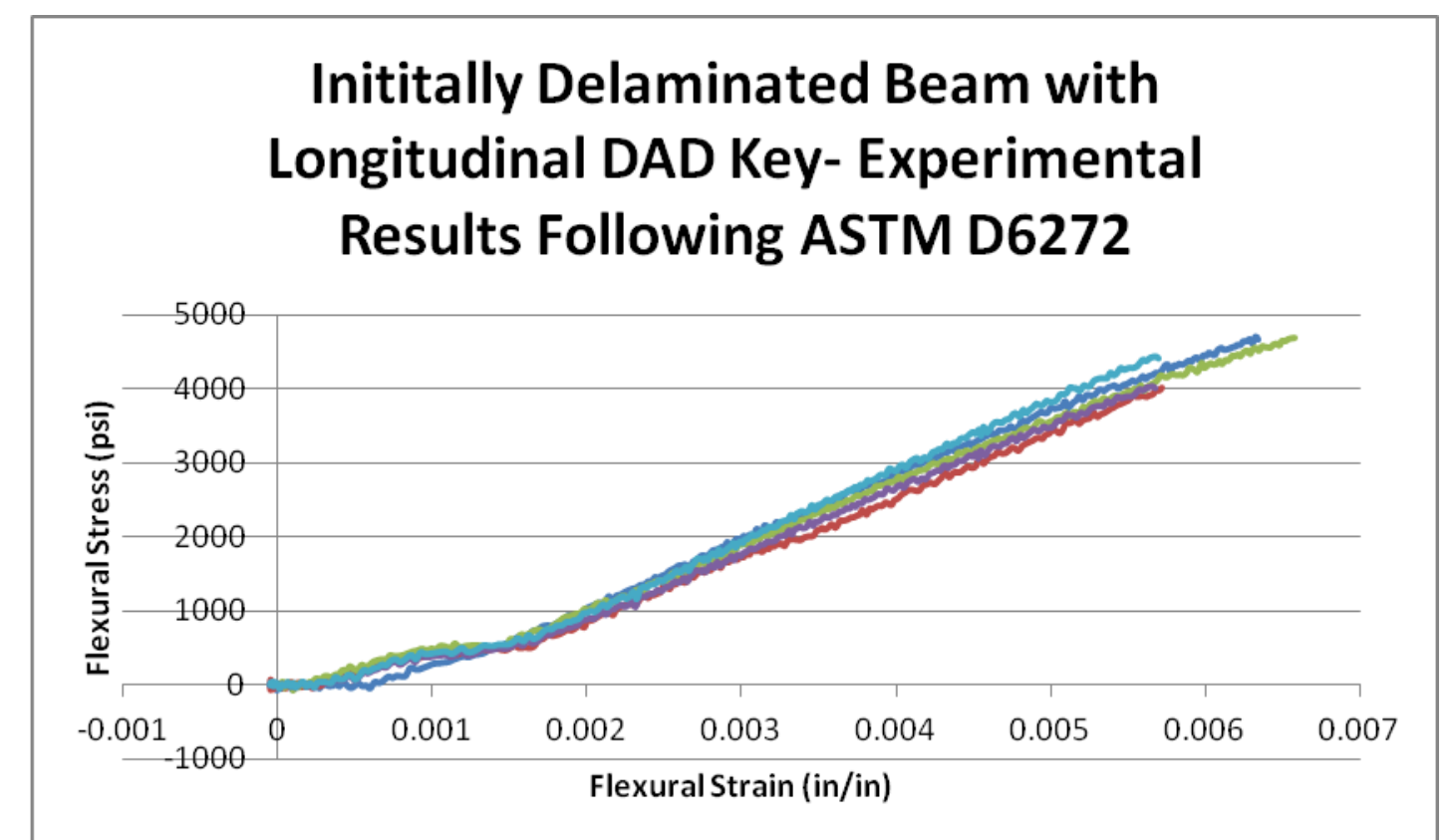

Figure 4.17. Resulting flexural stress-strain curves of longitudinal DAD key case group with an initial one inch long delamination subjected to static 4-point bending.

\subsubsection{Comparison of Composite Sandwich Beam Test Results}

Ultimately, the addition of DAD keys to delaminated beams greatly increased the structural integrity and ultimate strength of the beams. However, contrary to the delaminated case beams, the non-delaminated beams had a decrease in ultimate strength of $17 \%$ and $19 \%$ with the addition of the transverse and longitudinal DAD keys, respectively. A summary of all results of the sandwich beams tested under static 4-point bend can be seen in table 4.1. This table compares the average ultimate loads, ultimate stresses, elastic moduli, and percent differences from control beams.

Table 4.1. Results from experimentally investigated static 4-point bend testing for all test cases.

\begin{tabular}{|c|c|c|c|c|c|c|}
\hline & & Ultimate Load (lb) & $\begin{array}{l}\text { Ómax,f } \\
\text { (psi) }\end{array}$ & $\begin{array}{c}E_{B} \\
(M s i)\end{array}$ & $\begin{array}{c}\text { Ultimate } \\
\text { Strength } \\
\text { Increase over } \\
\text { Control } \\
(\%) \\
\end{array}$ & $\begin{array}{c}\text { Ultimate } \\
\text { Strength } \\
\text { Increase over } \\
\text { Delaminated } \\
\text { Control (\%) }\end{array}$ \\
\hline \multirow{2}{*}{$\begin{array}{l}\text { Control with no } \\
\text { DAD key }\end{array}$} & $\begin{array}{c}\text { Non } \\
\text { Delaminated }\end{array}$ & 253.15 & 7,364 & 0.816 & 0 & 147 \\
\hline & Delaminated & 102.58 & 2,984 & 0.585 & -60 & 0 \\
\hline \multirow{2}{*}{$\begin{array}{c}\text { Transverse DAD } \\
\text { key }\end{array}$} & $\begin{array}{c}\text { Non } \\
\text { Delaminated }\end{array}$ & 210.89 & 6,135 & 0.643 & -17 & 106 \\
\hline & Delaminated & 220.44 & 6,413 & 0.617 & -13 & 115 \\
\hline \multirow[t]{2}{*}{ Longitudinal key } & $\begin{array}{c}\text { Non } \\
\text { Delaminated }\end{array}$ & 204.40 & 5,946 & 0.872 & -19 & 99 \\
\hline & Delaminated & 154.36 & 4,491 & 0.573 & -39 & 66 \\
\hline
\end{tabular}


Through experimentation, it was found that adding DAD keys to the initially delaminated sandwich beams significantly increased the ultimate strength of the delaminated control beam by $147 \%$ for transversely oriented DAD keys and $66 \%$ for a longitudinally oriented DAD key. The addition of surface area bonding between the core and face sheet is the reason for the increase in ultimate strength. However, without any additional sheet resin added, the beams perform poorly without any initial delamination and added DAD keys because the stiffness of the beam is greatly increased. This is because adding a layer of sheet resin, seen in Davis et al., created a better bond between the face sheet and core, which in turn prolonged the delamination propagation. In both cases, failure was due to face core delamination, but it was clear that propagation of the initial delamination began shortly after the load was applied to the beams in this research.

It can be concluded that the addition of DAD keys can significantly improve the ultimate strength of a composite sandwich beam that has been initially delaminated. However, adding DAD keys to composite sandwich beams that have no initial delamination does not enhance the ultimate strength or the overall structural integrity. Without the addition of sheet resin between the core and face sheets, face core delamination is the main mode of failure, even with the addition of DAD keys. Unfortunately, this critically influences the amount of load the structure can take before failure. 


\subsection{Impact Test}

\subsubsection{Control Beam}

Five control test specimens manufactured using the autoclave method discussed in section 2.1 were tested according to test standard ASTM D7136. Instantaneous velocity at impact, force vs. time history, and acceleration-time history of the impact event were obtained through the Dynatup DAQ software, discussed in section 3.2. Each test specimen was subjected to a drop weight impact test by a 7.56-lb impactor dropped from an initial resting height of 5.5inches above the specimen. From the testing software, it was determined that the average impact velocity was 4.24 -ft/s with a standard deviation of $\pm 5.59 \%$, and the average maximum impact force experienced by the test specimen was 45.07 - Ib with a standard deviation of $\pm 3.59 \%$. The measured instantaneous velocity at impact is similar to the value found through classical free fall kinematics, with the experimentally obtained value reaching $78.0 \%$ of the theoretical $5.43-\mathrm{ft} / \mathrm{s}$ velocity from free fall at a 5.5-inch initial height. The small difference between the experimental and theoretical velocity values can be due to friction found between the crosshead and rails.

After the test specimen was impacted, the specimen was removed from the support fixture and the impact penetration was measured using a Fowler dial depth gauge. As shown in fig. 4.18, the impactor tup caused slight matrix and fiber breakage on each test specimen. However, there were no signs of tup penetration through the top face sheet. The impact penetration ranged from 0.034 to 0.040 -inches. 


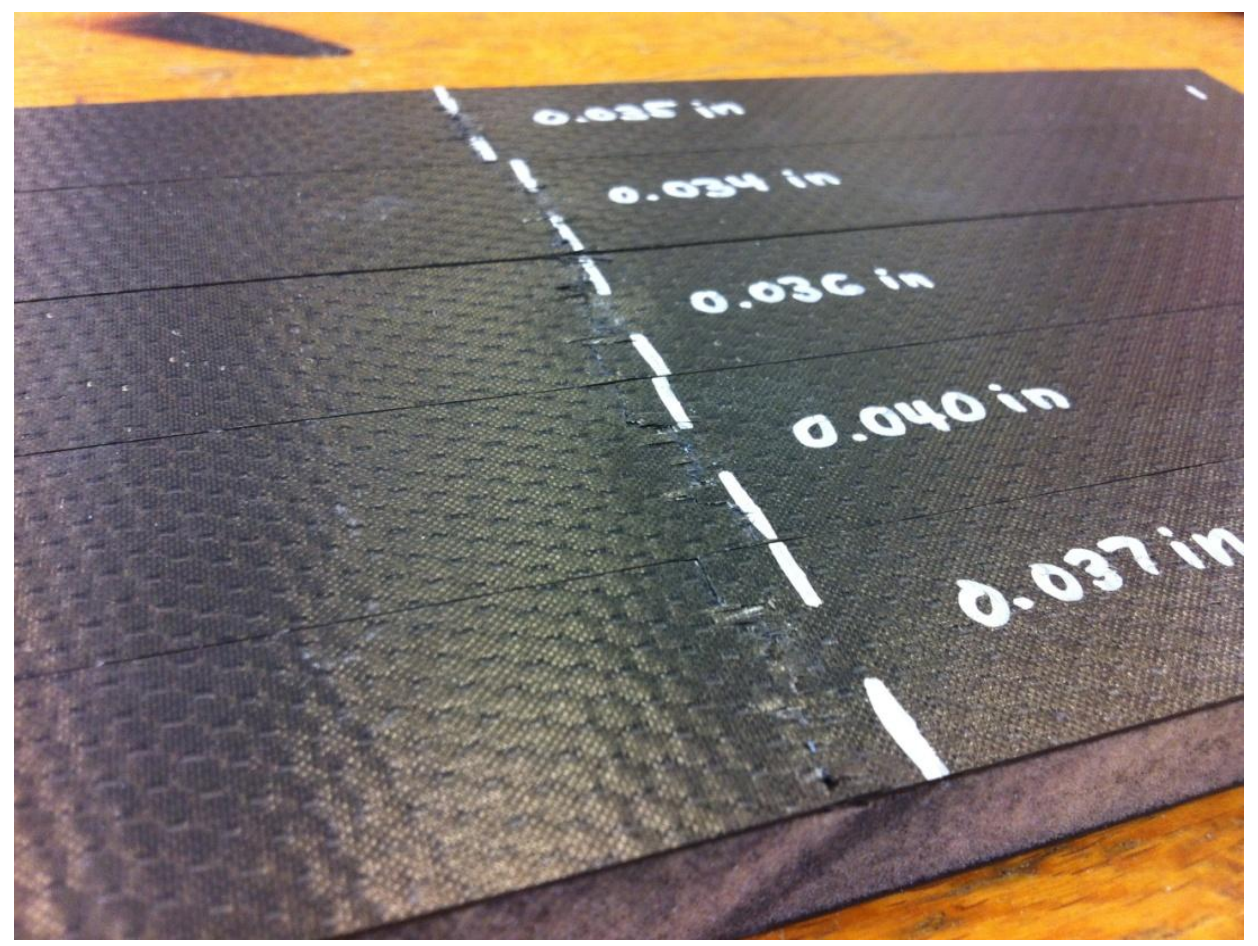

Figure 4.18. beams with no initial delamination or DAD key Post-impact.

\subsubsection{Transverse DAD key Beam}

Next, five sandwich beam test specimens with transverse DAD keys, manufactured using the autoclave method, were tested according to test standard ASTM D7136. Again, instantaneous velocity at impact, force vs. time history, and acceleration-time history of the impact event were obtained through the Dynatup DAQ software. Each test specimen was subjected to a drop weight impact test by a 7.56-lb impactor dropped from an initial resting height of 5.5-inches above the specimen. From the testing software, it was determined that the average impact velocity was $4.74-\mathrm{ft} / \mathrm{s}$ with a standard deviation of $\pm 9.55 \%$, and the average maximum impact force experienced by the test specimen was 48.91 - lb with a standard deviation of $\pm 3.26 \%$. The experimentally obtained instantaneous velocity value reached $87.3 \%$ of the theoretical 5.43 $\mathrm{ft} / \mathrm{s}$ velocity from free fall at a 5.5-inch initial height, where any differences were most likely due to friction found between the crosshead and rails.

After the test specimen was impacted, the specimen was removed from the support fixture and the impact penetration was measured. As shown in fig. 4.19, the impactor tup caused slight matrix breakage on each test specimen. There were no signs of matrix breakage or tup 
penetration through the top face sheet. The impact penetration ranged from 0.034 to 0.041 inches.

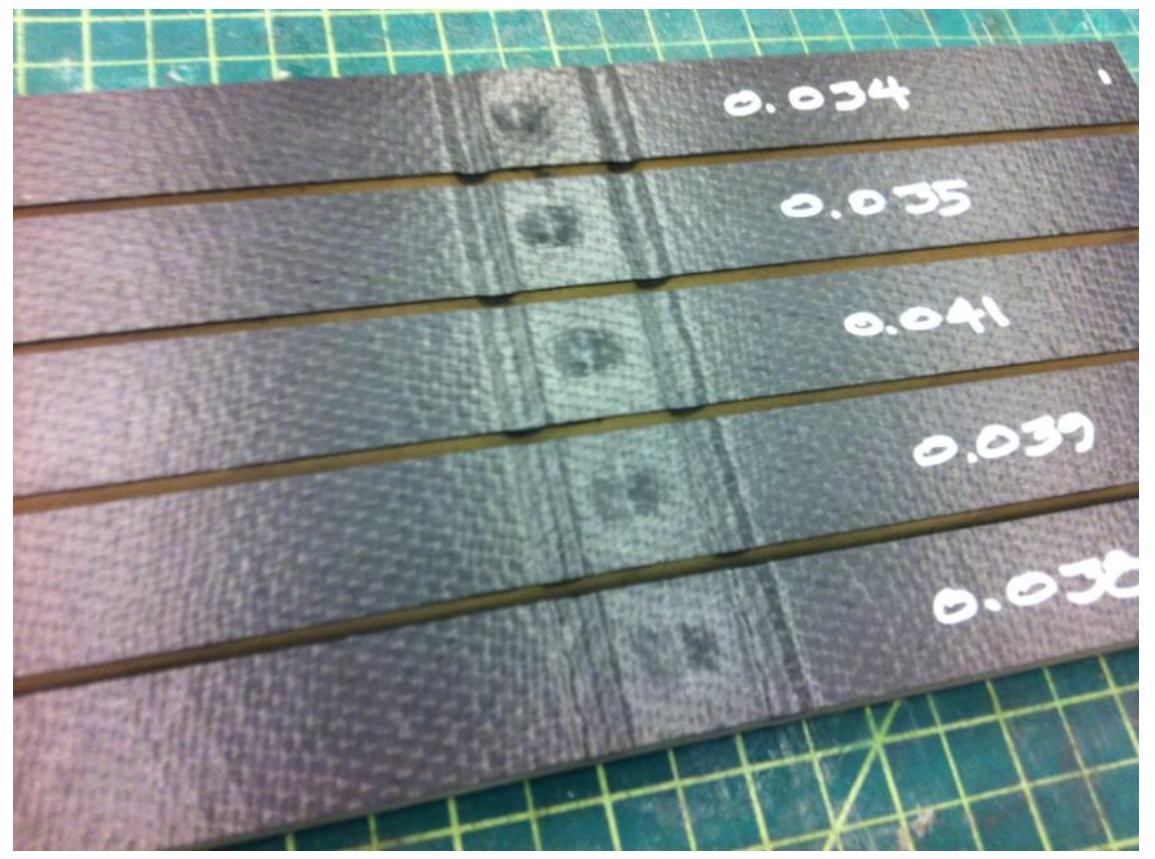

Figure 4.19. Composite sandwich beams with transverse DAD keys and no initial delamination Post-impact.

\subsubsection{Longitudinal DAD key Beam}

Five test specimens with an added longitudinal DAD key were tested according to test standard ASTM D7136. Each test specimen was subjected to a drop weight impact test by a 7.56-lb impactor dropped from an initial resting height of 5.5-inches above the specimen, similar to the previous control cases. From the Dynatup DAQ testing software, it was determined that the average impact velocity was 4.28 -ft/s with a standard deviation of $\pm 4.32 \%$, and the average maximum impact force experienced by the test specimen was 45.35 - Ib with a standard deviation of $\pm 3.36 \%$. The experimentally obtained instantaneous velocity value reached $78.9 \%$ of the theoretical $5.43-\mathrm{ft} / \mathrm{s}$ velocity from free fall at a 5.5 -inch initial height. Similar to the previous control cases, discrepancies found were likely due to friction found between the crosshead and rails.

Figure 4.20 depicts the longitudinal DAD key beam cases with no initial delamination post-impact. As shown, the impactor tup caused no matrix or fiber breakage on the test specimens. Since the longitudinal keys added stiffness to each test specimen, the impact did very little damage to the specimen. During testing, the sound of impact was very different from the 
sound found in the previous two cases, where the impactor tup rebounded very quickly and more frequently after the initial impact event. The impact penetration ranged from 0.005 to 0.009 inches.

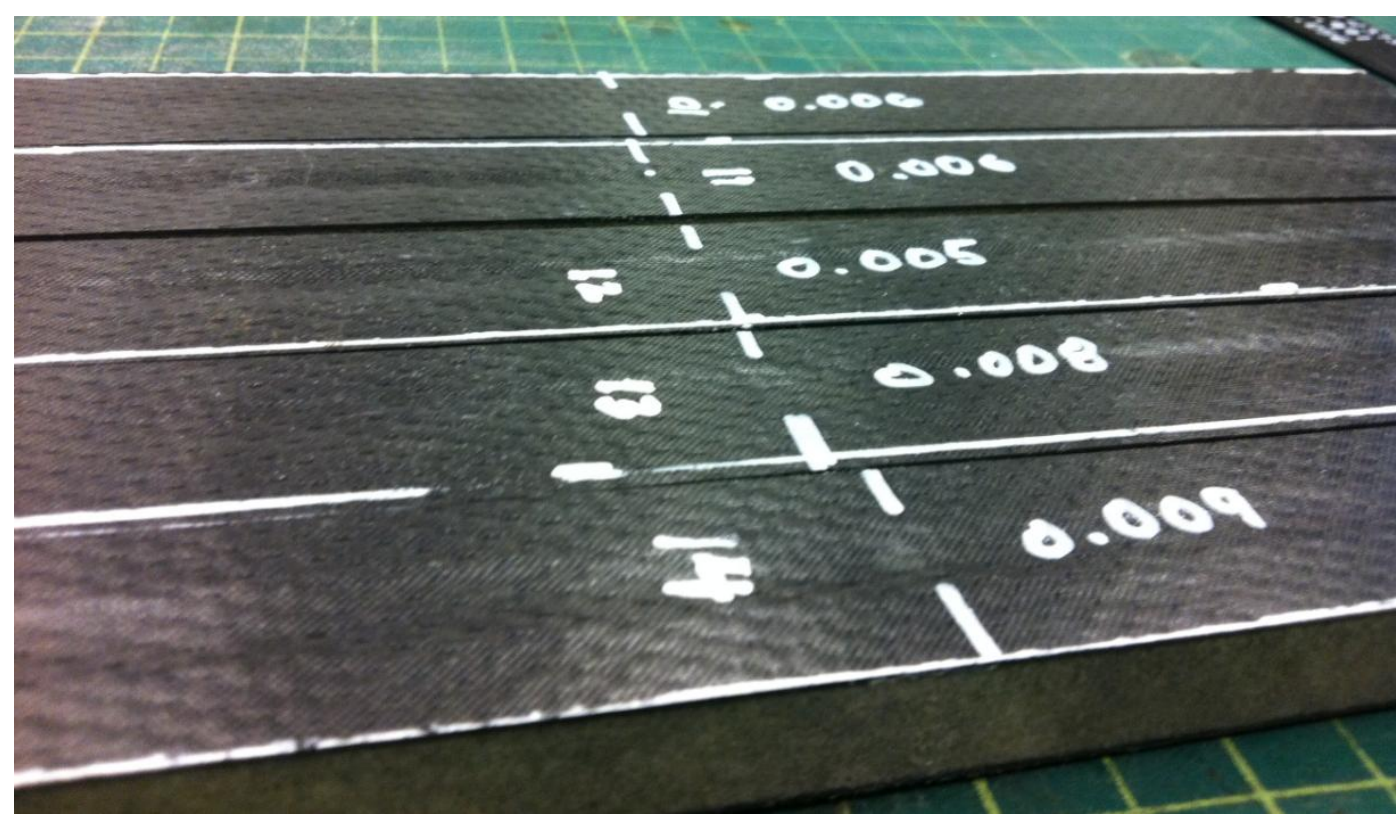

Figure 4.20. Composite sandwich beams with Longitudinal DAD key and no initial delamination Post-impact.

\subsection{4-Point Bend Fatigue Test}

1 kN Load Cell

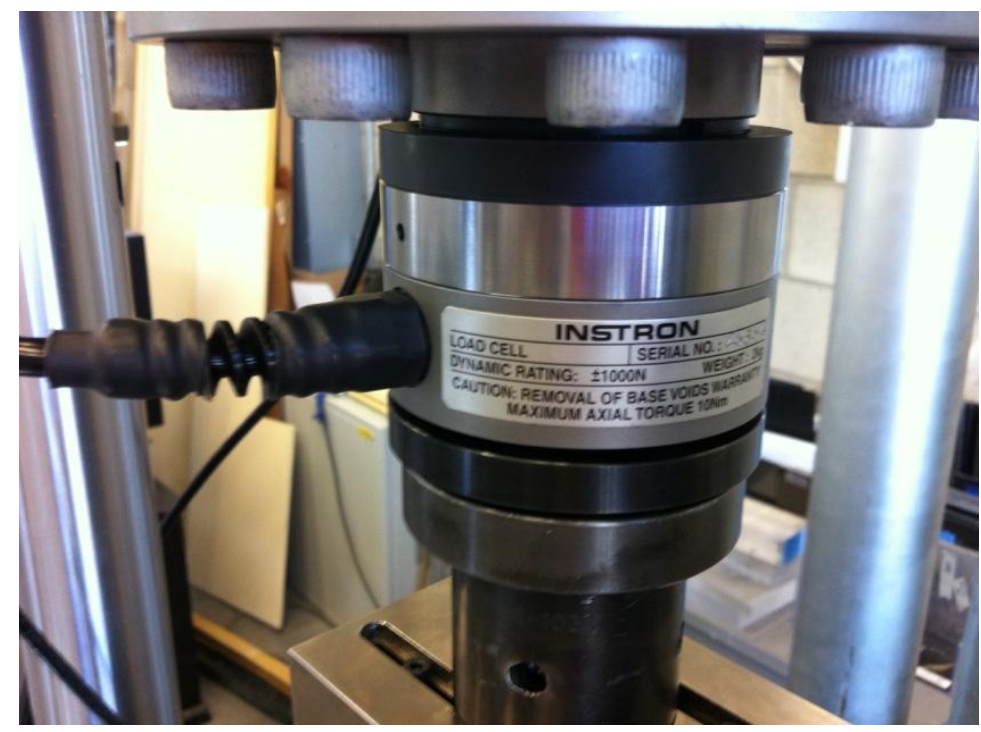


Figure 4.21. 1-kN load cell used for 4-point bend fatigue testing.

Fatigue tests utilizing the $1 \mathrm{kN}$ load cell were only conducted on the control case test specimen beams with no DAD keys or initial delamination. In total, five specimens were subjected to 4-point bend fatigue using the Instron 8801 Servohydraulic Fatigue Testing System in the Cal Poly Aerospace Structures/Composites laboratory. Unlike the static 4-point bend test, the 4-point bend fatigue test required a smaller load cell rated to $\pm 1 \mathrm{kN}$, shown in fig. 4.21. Changing the load cell allowed for more accurate yielded load values incurred on the test specimen beams, because of the small amplitude applied to the test specimens. Similar to the static 4-point bend testing, the test specimens were loaded in the 4-point bend jig, where the beam was centrally located between the supports. Figure 4.22 shows a test specimen beam secured in the testing support fixture prior to fatigue test. Instron WaveMatrix software was used to control the Instron 8801 servo-hydraulic testing system. The software used for fatigue testing yielded the number of cycles the test specimen beam could withstand before failure, maximum/minimum load, and maximum/minimum deflection. The control test specimen beams were ramped to a mean load and then subject to a dynamic loading at constant amplitude and frequency.

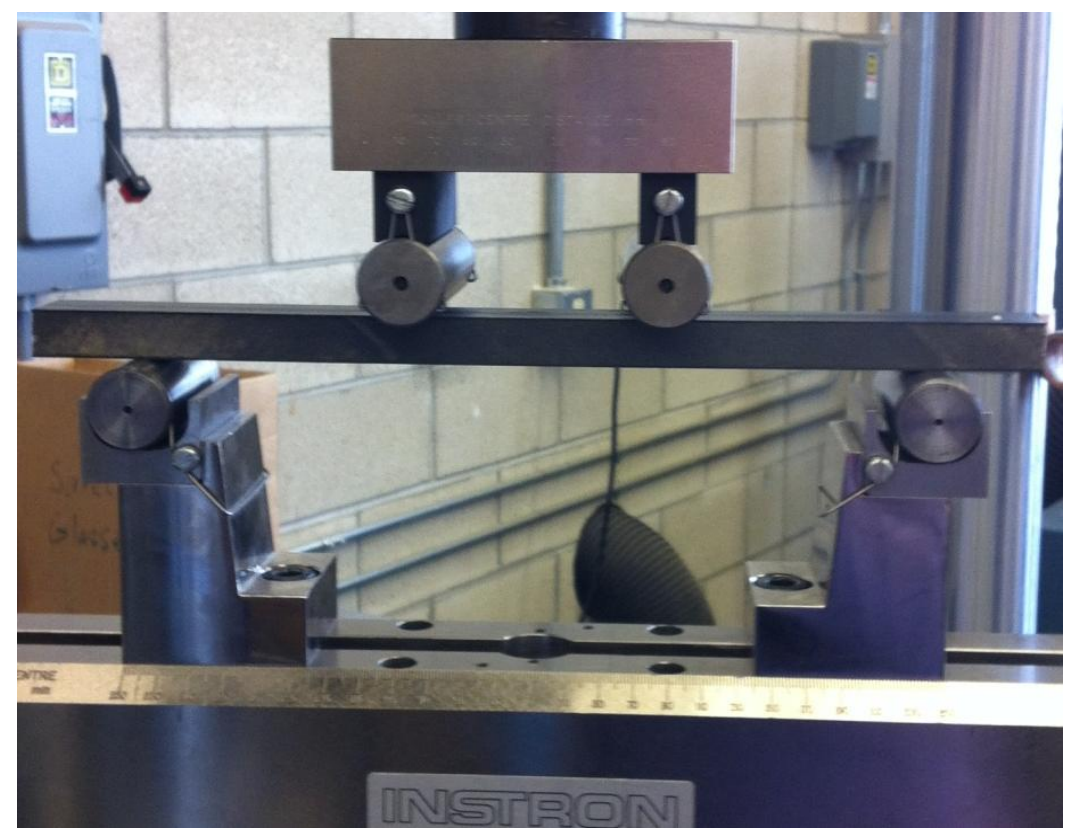

Figure 4.22. Control test specimen secured in 4-point bend jig for dynamic loading test.

The mean load used for fatigue testing chosen for this test case is $65 \%$ of the ultimate load of the test specimen and the amplitude was chosen to be $20 \%$ of the mean load. These 
values were determined to be $160 \mathrm{lbs}$ and $30 \mathrm{lbs}$, respectively. Dynamic flexural loading was performed at a frequency of $5 \mathrm{~Hz}$. This frequency was chosen because the lower the frequency, the more accurate the results are. Further, a lower frequency better models the environments to which structures are exposed to.

The results from the fatigue tests performed on the control test specimen beams are shown below in table 4.2. As shown, the average number of cycles to failure was 3,323 cycles with a percent error of $37.40 \%$. The variance in part life at similar load levels can be attributed to uncontrollable microscopic defects of different sizes and variations in each test group. In fatigue testing, the scatter of data at similar load levels is common.

Table 4.2. Fatigue test results of control test beams using $1 \mathrm{kN}$ load cell.

\begin{tabular}{|c|c|c|}
\hline Test Specimen & $\mathrm{N}_{\mathrm{f}}$ (Cycles to Failure) & \% $\mathrm{F}_{\text {ult }}$ \\
\hline 1 & 2,995 & $65 \%$ \\
\hline 2 & 3,169 & $65 \%$ \\
\hline 3 & 2,772 & $65 \%$ \\
\hline 4 & 2,225 & $65 \%$ \\
\hline 5 & 5,453 & $65 \%$ \\
\hline \multirow{2}{*}{ With Outliers } & Average $\mathrm{N}_{\mathrm{f}}$ & 3,323 \\
\cline { 2 - 4 } & Percent Error & $37.40 \%$ \\
\hline \multirow{2}{*}{ Without Outliers } & Average $\mathrm{N}_{\mathrm{f}}$ & 2,790 \\
\cline { 2 - 3 } & Percent Error & $14.71 \%$ \\
& & \\
\hline
\end{tabular}




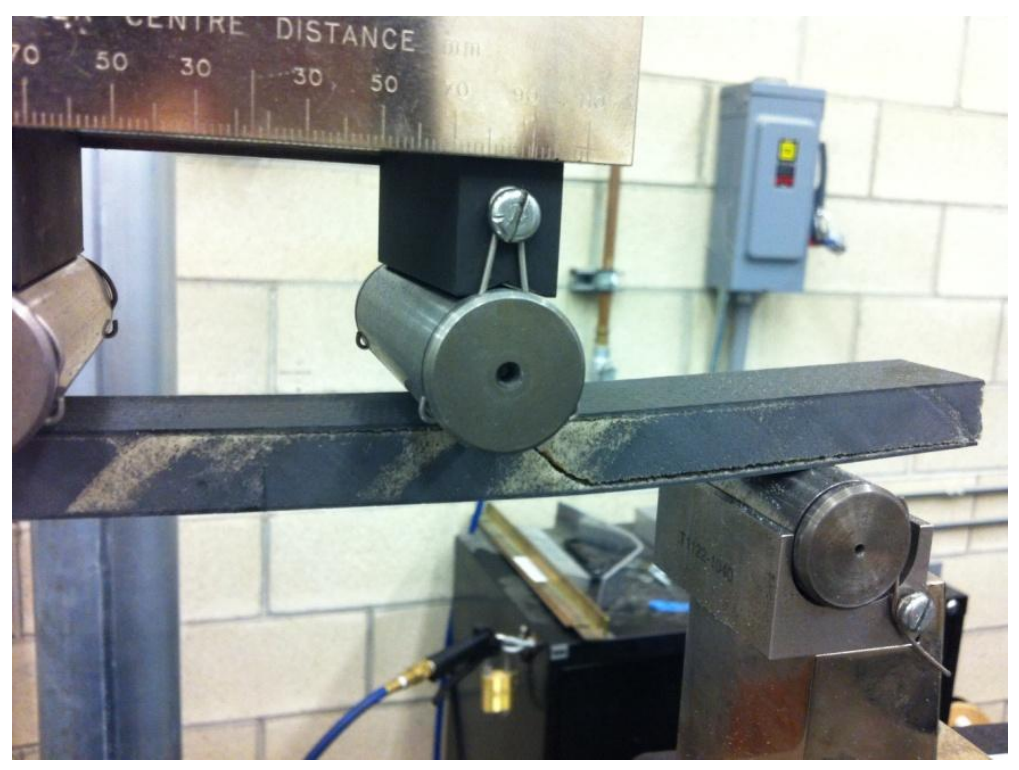

Figure 4.23. Typical failure from fatigue test on control beam with no initial delamination or DAD key.

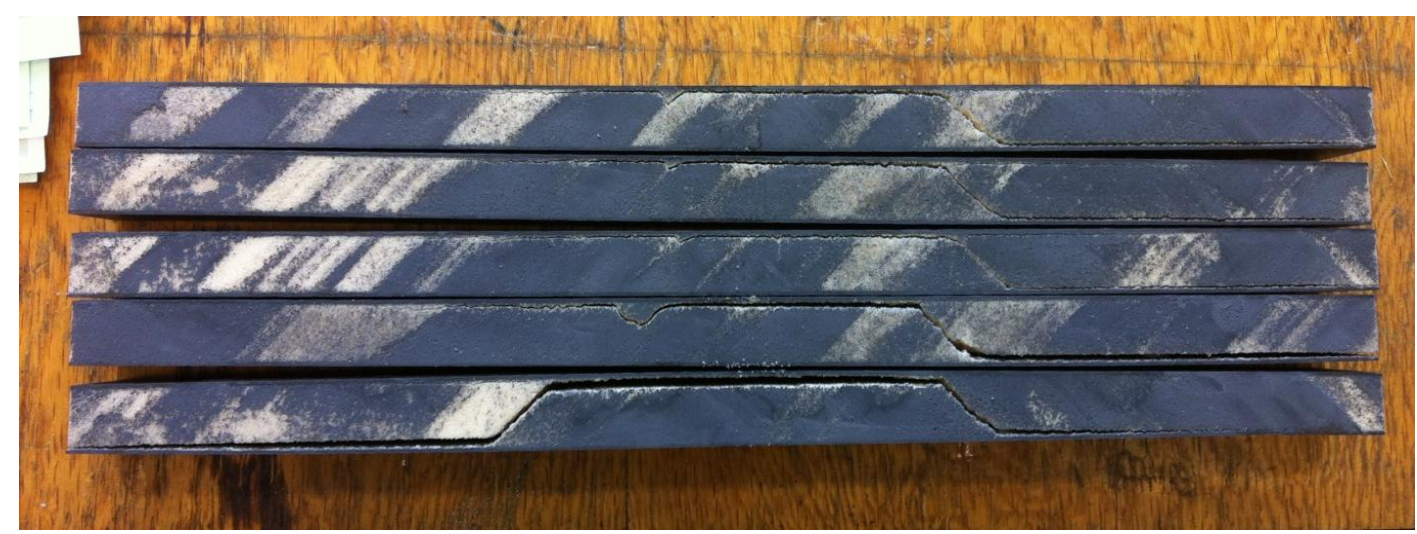

Figure 4.24. Control test group after final failure and completed fatigue life.

Despite varying fatigue life values in the control group, each test specimen failed very similarly. Figure 4.23 depicts the failure seen in the control beam upon the final life cycle. As shown, failure began with face-core delamination on the upper loaded face sheet over the left loaded cylinder and propagated approximately 2 inches before fracturing through the core. Complete fracture propagated through the depth of the core and subsequent face-core delamination on the support end continued through to the right end of the beam. All of the tested specimens mainly failed at the right support which may indicate that there is a very slight offalignment of the testing fixture. No failure was visible in the composite face sheet, which indicates that the flexural strength of the control case beam was based upon the flexural strength of the foam core and the shear strength of the face-core bond. Figure 4.24 shows the control test 
beams after final failure, which shows that the failure was almost identical throughout the test group.

\section{0 kN Load Cell}

A $100 \mathrm{kN}$ load cell was implemented in fatigue tests due to a malfunction of the $1 \mathrm{kN}$ load cell, as discussed in section 3.3. Fatigue loading was conducted on the following cases: control case test specimen beams with no DAD keys or initial delamination, beams with transverse DAD keys and no initial delamination, beams with longitudinal DAD keys and no initial delamination, initially delaminated test case beams with no DAD keys, initially delaminated test case beams with transverse DAD keys, and initially delaminated test case beams with a longitudinal DAD keys. Additionally, previously impacted beams were also fatigued to obtain each subsequent fatigue life value, and to determine whether DAD keys increased the fatigue life of an impacted test specimen. Post-impact cases tested included: control case test specimen beams with no DAD keys or initial delamination, beams with transverse DAD keys and no initial delamination, and beams with longitudinal DAD keys and no initial delamination. For each case, five specimens were subjected to 4-point bend fatigue testing using the Instron 8801 Servohydraulic Fatigue Testing System. Although, with the initial settings, the $100 \mathrm{kN}$ load cell did not yield as precise load readings as the $1 \mathrm{kN}$ load cell, the control loop proportional gain of the load cell was increased significantly to provide accurate load values incurred on the test specimen. Further, the frequency was decreased from $5 \mathrm{~Hz}$ to $1 \mathrm{~Hz}$. Similar to the static 4-point bend testing and the fatigue testing using the $1 \mathrm{kN}$ load cell, the test specimens were loaded in the 4-point bend jig, where the beam was centrally located between the supports.

\subsubsection{Control Case}

The control case with no delamination or DAD key was the first tested under 4-point bend fatigue loading at a $1 \mathrm{~Hz}$ frequency. In total, five specimens were tested to a mean load of $65 \%$ of the ultimate load of the test specimen and amplitude of $20 \%$ of the mean load. These values were determined to be $160 \mathrm{lbs}$ and $30 \mathrm{lbs}$, respectively. As discussed in section 4.1, maximum load 
before failure for each test beam case was found through a static 4-point bend test. The results from the fatigue tests performed on the control test specimen beams are shown below in table 4.3. As shown, the average number of cycles to failure was 4,739 cycles with a percent error of $18.63 \%$. Again, the variance in part life at similar load levels can be attributed to uncontrollable microscopic defects of different sizes and variations in each test group and in fatigue testing, the scatter of data at similar load levels is common.

Table 4.3. Fatigue test results of control test beams using $100 \mathrm{kN}$ load cell.

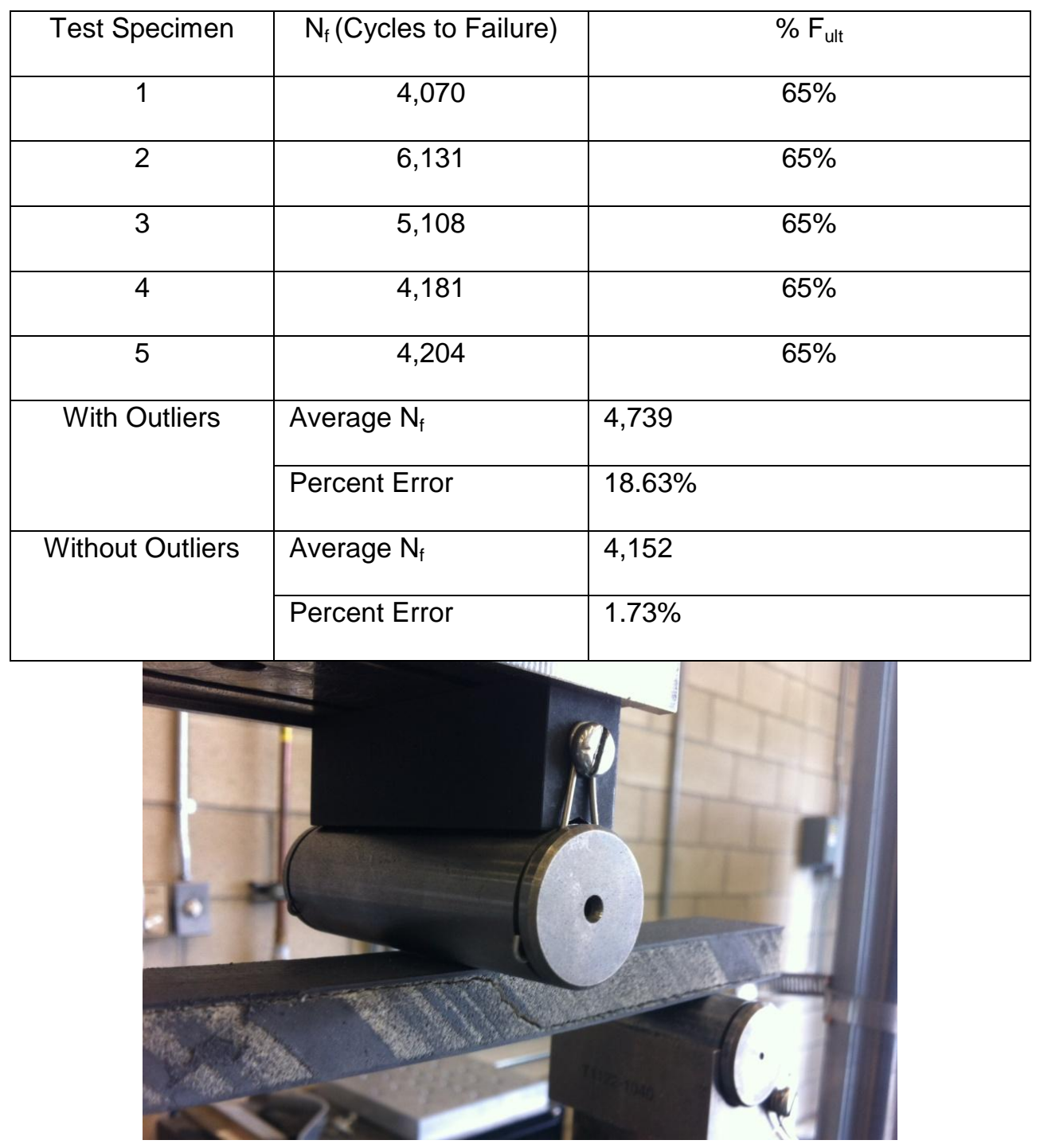

Figure 4.25. Typical failure from fatigue test at $1 \mathrm{~Hz}$ on control beam with no initial delamination or DAD key.

Despite the varying fatigue life values in the control group, each test specimen failed very similarly. Figure 4.25 depicts the failure seen in the control beam, under a $1 \mathrm{~Hz}$ dynamic 4-point 
bend, upon the final life cycle. As shown, failure began with face-core delamination on the upper loaded face sheet over the left loaded cylinder and propagated approximately 2 inches before fracturing through the core. Complete fracture propagated through the depth of the core and subsequent face-core delamination on the support end continued through to the right end of the beam. All of the tested specimens ultimately failed at the right support which may indicate that there is a very slight off-alignment of the testing fixture. No failure was visible in the composite face sheet, which indicates that the flexural strength of the control case beam was based upon the flexural strength of the foam core and the shear strength of the face-core bond. All control test specimens without DAD keys and no initial delamination ultimately fractured through the core at the same location, under the right loaded cylinder, and can be seen below in fig. 4.26.

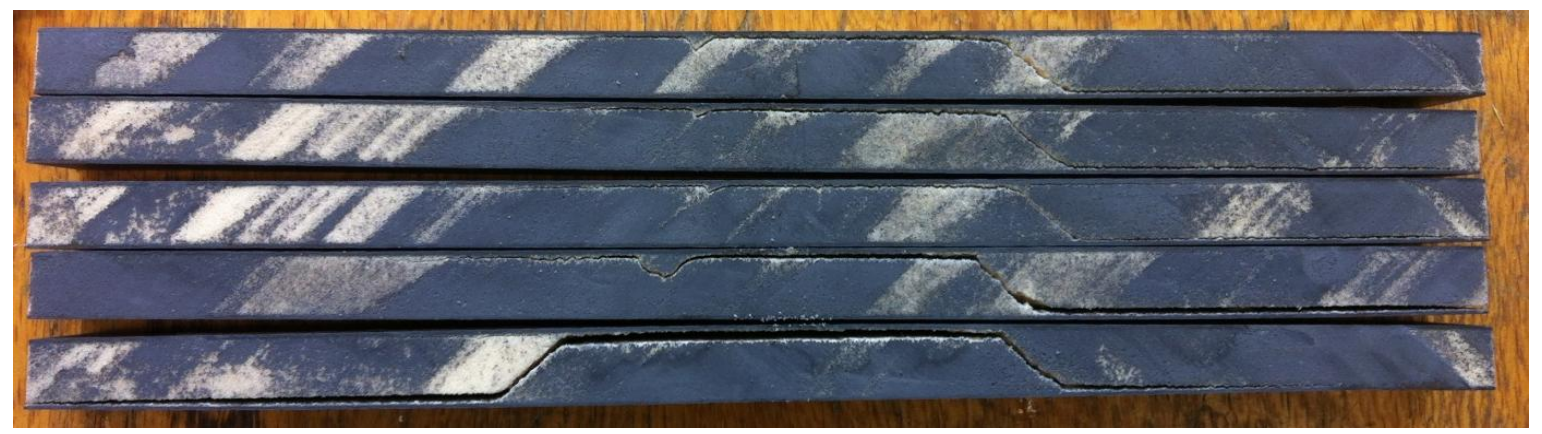

Figure 4.26. Control test group, tested with $100 \mathrm{kN}$ load cell, after final failure and completed fatigue life.

After testing the control group with no DAD key or initial delamination under only 4-point bend fatigue, the beams which were previously impacted were tested. All beams were fatigued with 4-point bend loading immediately after impact testing to ensure that no creep or relaxation effects due to impact would occur. Five impacted test specimens were tested under the same fatigue test conditions as the non-impacted test specimens. The test specimens were tested to a mean load of $65 \%$ of the ultimate load of the impacted control test specimen and amplitude of $40 \%$ of the mean load. These values were determined to be $72 \mathrm{lbs}$ and $30 \mathrm{lbs}$, respectively. The results from the fatigue tests performed on the impacted control test specimen beams are shown below in table 4.4. As shown, the average number of cycles to failure was 3,368 cycles with a percent error of $80.03 \%$. The large variations in fatigue life are likely due to the inconsistency in impact penetrations across the test specimens, which affects the test specimens' capability to withstand the dynamic loading. 
Table 4.4. Fatigue test results of impacted control test beams using $100 \mathrm{kN}$ load cell.

\begin{tabular}{|c|c|c|}
\hline Test Specimen & $\mathrm{N}_{\mathrm{f}}$ (Cycles to Failure) & $\% \mathrm{~F}_{\text {ult }}$ \\
\hline 1 & 7,522 & $65 \%$ \\
\hline 2 & 2,804 & $65 \%$ \\
\hline 3 & 721 & $65 \%$ \\
\hline 4 & 1,476 & $65 \%$ \\
\hline \multirow{2}{*}{ With Outliers } & 4,316 & $65 \%$ \\
\cline { 2 - 3 } & Average $\mathrm{N}_{\mathrm{f}}$ & 3,368 \\
\cline { 2 - 3 } & Percent Error & $80.03 \%$ \\
\hline \multirow{2}{*}{ Without Outliers } & Average N & 1,667 \\
\cline { 2 - 3 } & Percent Error & $63.26 \%$ \\
& & \\
\hline
\end{tabular}

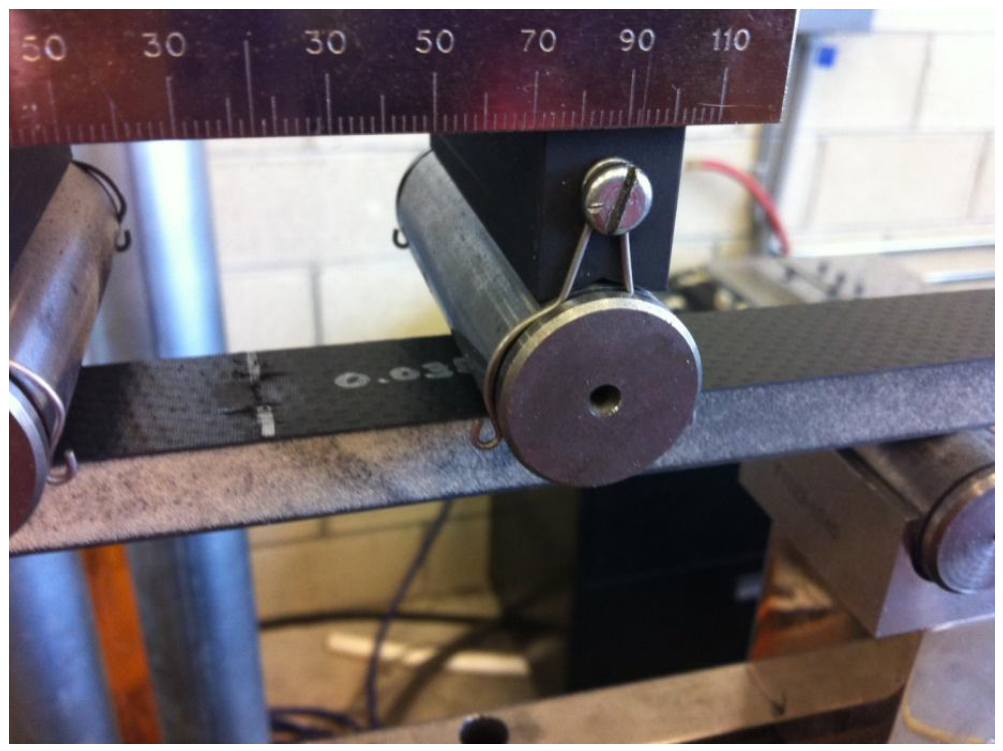

Figure 4.27. Typical failure from fatigue test at $1 \mathrm{~Hz}$ on previously impacted control beam with no initial delamination or DAD key.

Although the fatigue life of each test specimen differed, each test specimen failed identically. Figure 4.27 depicts the failure seen in the impacted control beam, under a $1 \mathrm{~Hz} 4$ point bend fatigue loading, upon the final life cycle. As shown, the impacted control beam failed due to matrix and fiber cracking. As shown in fig. 4.28 , all of the tested specimens ultimately failed due to a complete face sheet fracture that did not propagate any further than the top face sheet. In this case, the initial fiber breakage due to impact caused the test beam to fail 
prematurely. Therefore, flexural strength of the impacted control case beam was based upon the flexural strength of the face sheet.

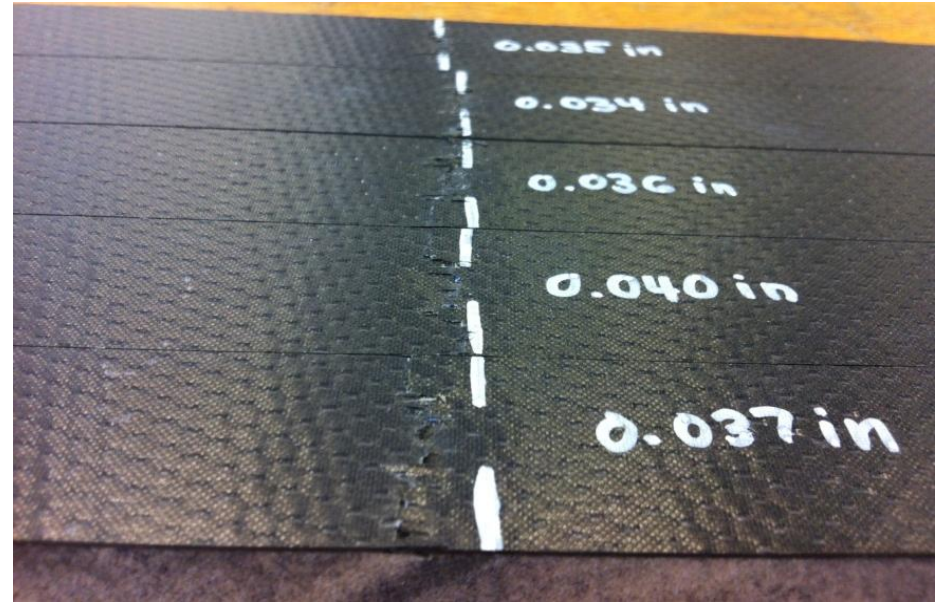

Figure 4.28. Impacted Control test group tested with $100 \mathrm{kN}$ load cell, after final failure and completed fatigue life.

\subsubsection{Beam with Transverse DADs}

The composite sandwich beam case with no delamination and transverse DAD keys was also tested under 4-point bend fatigue conditions at a $1 \mathrm{~Hz}$ frequency. In total, five specimens were tested to a mean load of $65 \%$ of the ultimate load of the control test specimen with no initial delamination or DAD key and amplitude of $20 \%$ of the mean load. As stated previously, these values were determined to be $160 \mathrm{lbs}$ and $30 \mathrm{lbs}$, respectively. The results from the fatigue tests performed on the control test specimen beams with transverse DAD keys are shown below in table 4.5. The average number of cycles to failure was 6,282 cycles with a percent error of $51.06 \%$. The percent error including the outlier value is very high due to a very short life cycle of 2,091 cycles. This great variance can be attributed to many factors, but is most likely due to microscopic defects in the test specimen. However, with the same mean load value and amplitude as the control case, it can be seen that the average number of cycles to failure greatly increased with the addition of the transverse DAD keys. On average, the addition of the transverse DAD keys increased the number of cycles to failure by $32.56 \%$. This is contrary to the static 4-point bending case, where the addition of the transverse DAD keys caused a decrease in ultimate strength of $17 \%$.

Table 4.5. Fatigue test results of test beams with transverse DAD keys and no delamination. 


\begin{tabular}{|c|l|l|}
\hline Test Specimen & $\mathrm{N}_{\mathrm{f}}$ (Cycles to Failure) & \% $\mathrm{F}_{\text {ult }}$ \\
\hline 1 & 5,258 & $65 \%$ \\
\hline 2 & 5,833 & $65 \%$ \\
\hline 3 & 7,337 & $65 \%$ \\
\hline 4 & 2,091 & $65 \%$ \\
\hline 5 & 10,889 & $65 \%$ \\
\hline \multirow{2}{*}{ With Outliers } & Average $\mathrm{N}_{\mathrm{f}}$ & 6,282 \\
\cline { 2 - 4 } & & \\
\hline & Percent Error & $51.06 \%$ \\
\hline \multirow{2}{*}{ Without Outliers } & Average $\mathrm{N}_{\mathrm{f}}$ & 7,329 \\
\cline { 2 - 4 } & Percent Error & $34.52 \%$ \\
& & \\
\hline
\end{tabular}

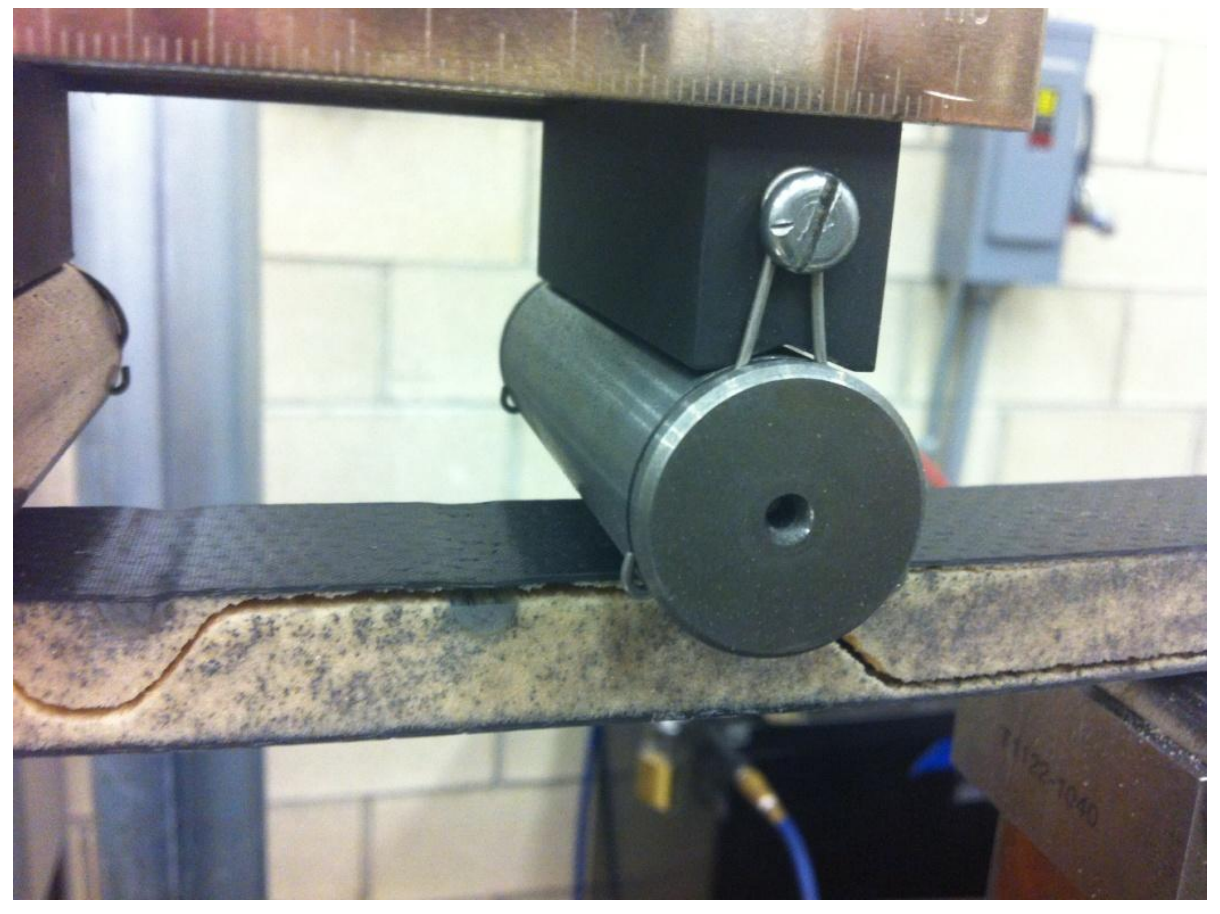

Figure 4.29. Typical failure from fatigue test at $1 \mathrm{~Hz}$ on beams with no initial delamination and transverse DAD keys.

The variance in fatigue life values in the group with added transverse DAD keys was very high, but each test specimen failed very similarly. The typical failure witnessed during 4-point bend fatigue testing can be seen in fig. 4.29. As shown above, the failure mode observed began with a similar face-core interface failure and foam fracture as the control case with no 
delamination or DAD. However, in this case the shear key delayed fracture through the core. The face-core delamination propagated both left and right of the loading cylinder, with fracture occurring under the right loaded cylinder, similar to what was observed in the control cases. As depicted in fig. 4.29, the DAD key arrested fracture through the foam core to the left of the right loaded cylinder and face-core delamination continued around the DAD key and further propagated between the upper face sheet and core. This again indicated that the flexural strength of the sandwich beam was driven by the flexural strength of the foam core and shear strength of the face-core bond. Figure 4.30 depicts similar ultimate failure between the beams with the addition of transverse DAD keys.

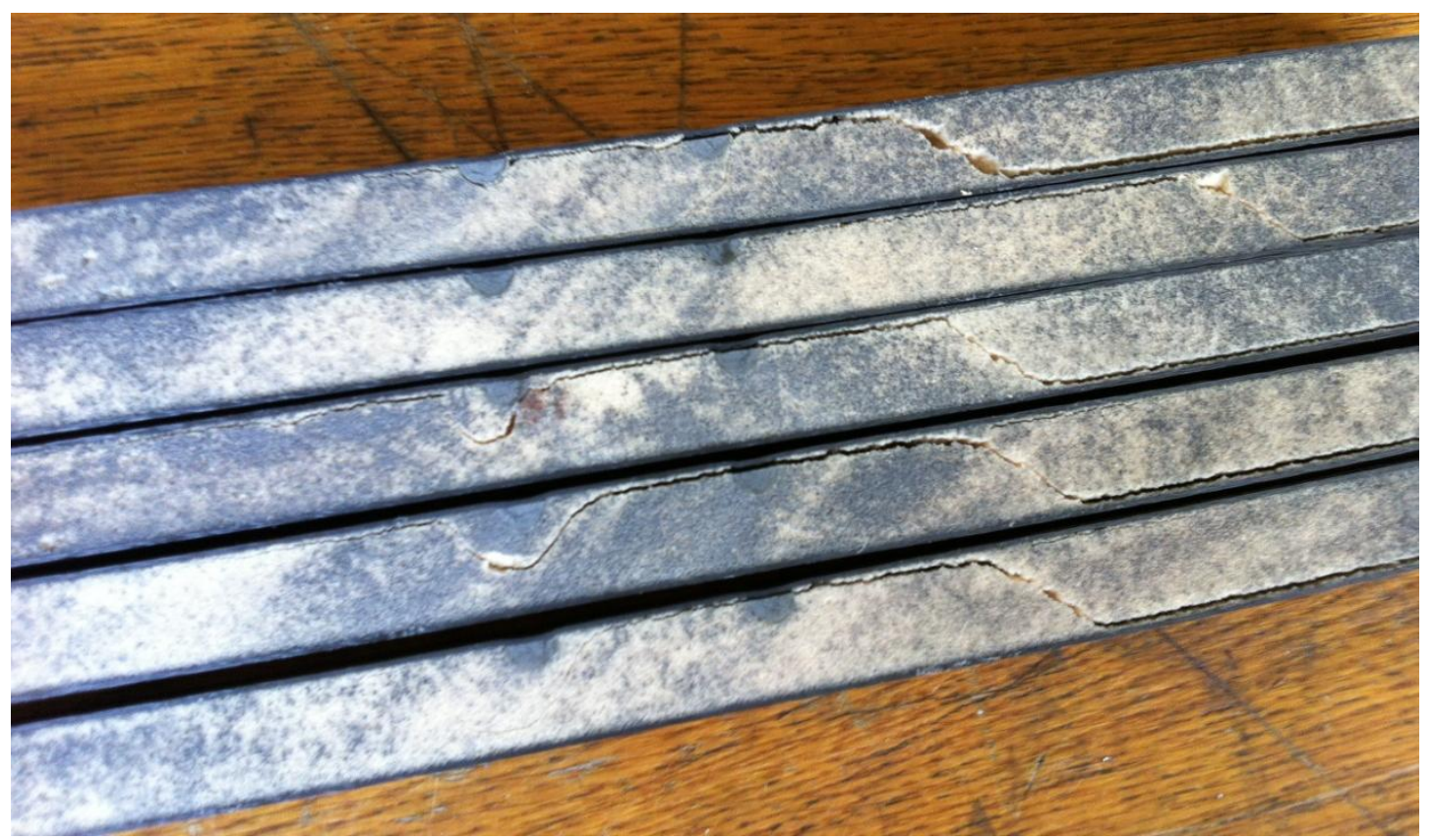

Figure 4.30. Test group with the addition of transverse DAD keys after final failure and completed fatigue life.

After testing the beams with transverse DAD keys and no initial delamination under only 4-point bend fatigue, the beams which were previously impacted were tested. Again, all beams were tested under 4-point bend fatigue conditions immediately after impact testing to ensure that no creep or relaxation effects due to impact would occur. Five impacted test specimens with added transverse DAD keys were tested under the same fatigue test conditions as the nonimpacted test specimens. The test specimens were tested to a mean load of $65 \%$ of the ultimate load of the impacted control test specimen and amplitude of $40 \%$ of the mean load. These values 
were determined to be $72 \mathrm{lbs}$ and $30 \mathrm{lbs}$, respectively. The results from the fatigue tests performed on the impacted test specimen beams with the addition of transverse DAD keys are shown below in table 4.6. As shown, the average number of cycles to failure was 24,737 cycles with a percent error of $90.83 \%$. The percent error is very high due to two specimens failing prematurely, but without including these test specimens, the average number of cycles to failure increases to 41,117 cycles with a percent error of $4.67 \%$. The higher average more accurately portrays the benefits of adding the transverse DAD keys to an impacted control beam. With the same mean load value and amplitude as the impacted control case, it can be seen that the average number of cycles to failure greatly increased with the addition of the transverse DAD keys. On average, the addition of the transverse DAD keys increased the number of cycles to failure by $634.48 \%$. However, the significant differences in fatigue life in this particular test group are generally due to inconsistent impact penetrations and uncontrollable microscopic defects of different sizes.

Table 4.6. Fatigue test results of impacted test beams with transverse DAD keys using 100kN load cell.

\begin{tabular}{|c|c|c|}
\hline Test Specimen & $\mathrm{N}_{\mathrm{f}}$ (Cycles to Failure) & $\% \mathrm{~F}_{\text {ult }}$ \\
\hline 1 & 38,999 & $65 \%$ \\
\hline 2 & 42,740 & $65 \%$ \\
\hline 3 & 133 & $65 \%$ \\
\hline 4 & 201 & $65 \%$ \\
\hline 5 & 41,613 & $65 \%$ \\
\hline \multirow{2}{*}{ With Outliers } & Average $\mathrm{N}_{\mathrm{f}}$ & 24,737 \\
\cline { 2 - 3 } & Percent Error & $90.83 \%$ \\
\hline \multirow{2}{*}{ Without Outliers } & Average $\mathrm{N}_{\mathrm{f}}$ & 41,117 \\
\cline { 2 - 3 } & Percent Error & $4.67 \%$ \\
& & \\
& & \\
& & \\
\end{tabular}




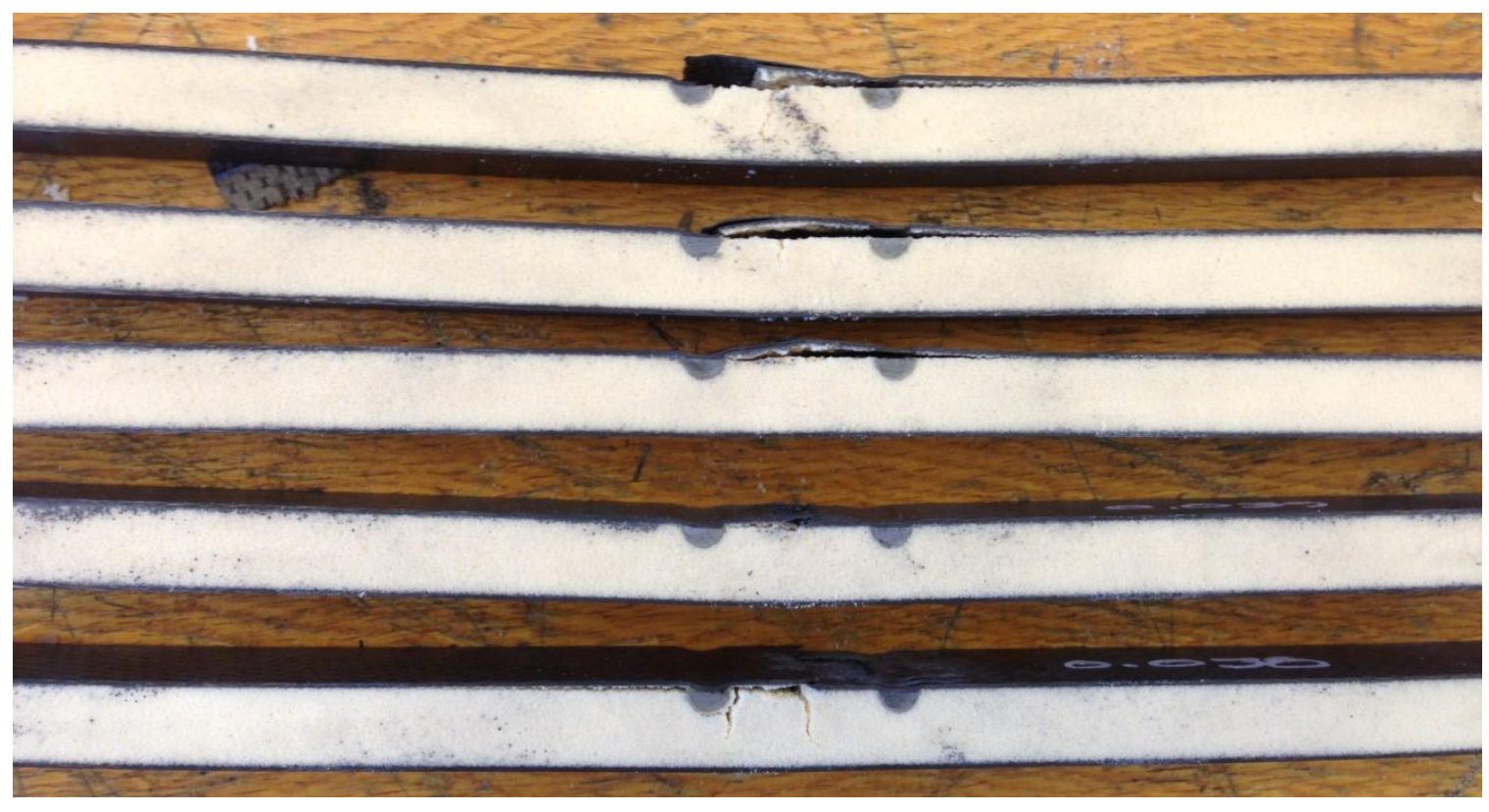

Figure 4.31. Typical failure from fatigue test at $1 \mathrm{~Hz}$ on previously impacted beams with no initial delamination and transverse DAD keys.

The fatigue life of each test specimen that did not fail prematurely was very consistent, including the type of failure. Figure 4.31 depicts the failure seen in the impacted beam with no initial delamination and added transverse DAD keys, under a $1 \mathrm{~Hz}$ dynamic 4-point bend. Similar to the impacted control case, the impacted control beams with transverse DAD keys failed due to matrix and fiber cracking, as shown in fig. 4.32. However, fig. 4.31 shows that the failure continued into the foam core and caused cracking in the foam. The initial fiber breakage due to impact caused some test beams to fail prematurely, and caused others to fail completely due to fiber cracking in the top face sheet. Therefore, this indicates that the flexural strength of the impacted test group with added transverse DAD keys was based upon the flexural strength of the top face sheet with the initial flaw due to impact. 


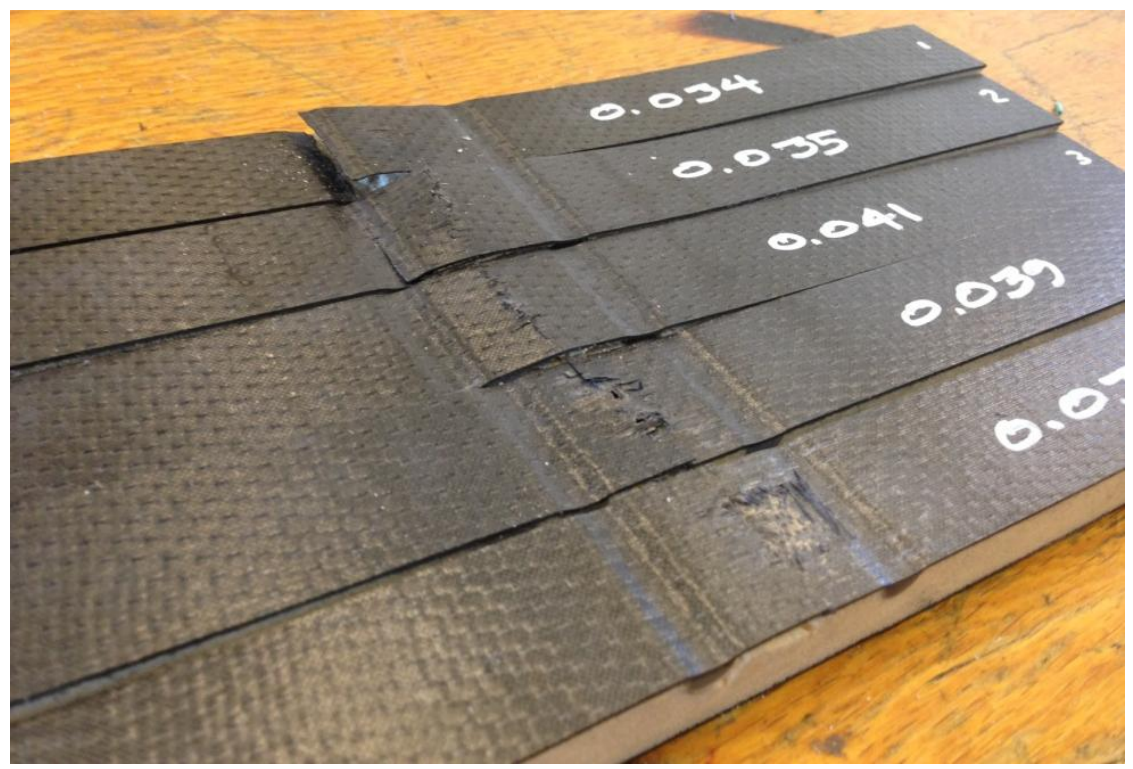

Figure 4.32. Impacted test group with added transverse DAD keys tested with $100 \mathrm{kN}$ load cell, after final failure and completed fatigue life.

\subsubsection{Beam with Longitudinal DAD}

The longitudinal DAD key case beam with no delamination was the third case tested under 4-point bend fatigue conditions at a $1 \mathrm{~Hz}$ frequency. Similar to the previous two cases, five specimens were tested to a mean load of $65 \%$ of the ultimate load of the control test specimen with no initial delamination or DAD key and amplitude of $20 \%$ of the mean load. As stated previously, these values were determined to be $160 \mathrm{lbs}$ and $30 \mathrm{lbs}$, respectively. The results from the fatigue tests performed on the beams with a longitudinal DAD key are shown below in table 4.7. The average number of cycles to failure was 1,460 cycles with a percent error of $65.14 \%$. The percent error including the outlier value is very high due to two short life cycles of 384 and 649 cycles. Without the outlying values, the average life cycle observed was 2,008 cycles with a percent error of $26.58 \%$. The premature failure seen in the two test specimens can be due to the fact that the longitudinal keys were not completely bonded to the core and face sheet, and acted more as a defect than an added surface area connection. Further, similar to the static 4-point bend test, the case with an added longitudinal key and no delamination failed much sooner than the case with no delamination or DAD key under 4-point bend fatigue testing. On average, the number of cycles to failure decreased by $69.19 \%$ from the control group case. This is likely due to 
the DAD acting more as a foreign object, which causes a different reaction to the constraints and makes the structure overall stiff.

Table 4.7. Fatigue test results of longitudinal DAD key beams with no delamination.

\begin{tabular}{|c|l|l|}
\hline Test Specimen & $\mathrm{N}_{\mathrm{f}}$ (Cycles to Failure) & \% $\mathrm{F}_{\text {ult }}$ \\
\hline 1 & 384 & $65 \%$ \\
\hline 2 & 1,450 & $65 \%$ \\
\hline 3 & 649 & $65 \%$ \\
\hline 4 & 2,358 & $65 \%$ \\
\hline 5 & 2,457 & $65 \%$ \\
\hline \multirow{2}{*}{ With Outliers } & Average $\mathrm{N}_{\mathrm{f}}$ & \\
\cline { 2 - 4 } & Percent Error & $65.14 \%$ \\
& & 2,088 \\
\hline \multirow{2}{*}{ Without Outliers } & Average $\mathrm{N}_{\mathrm{f}}$ & $26.58 \%$ \\
\cline { 2 - 4 } & & \\
\cline { 2 - 4 } & Percent Error & \\
\hline
\end{tabular}

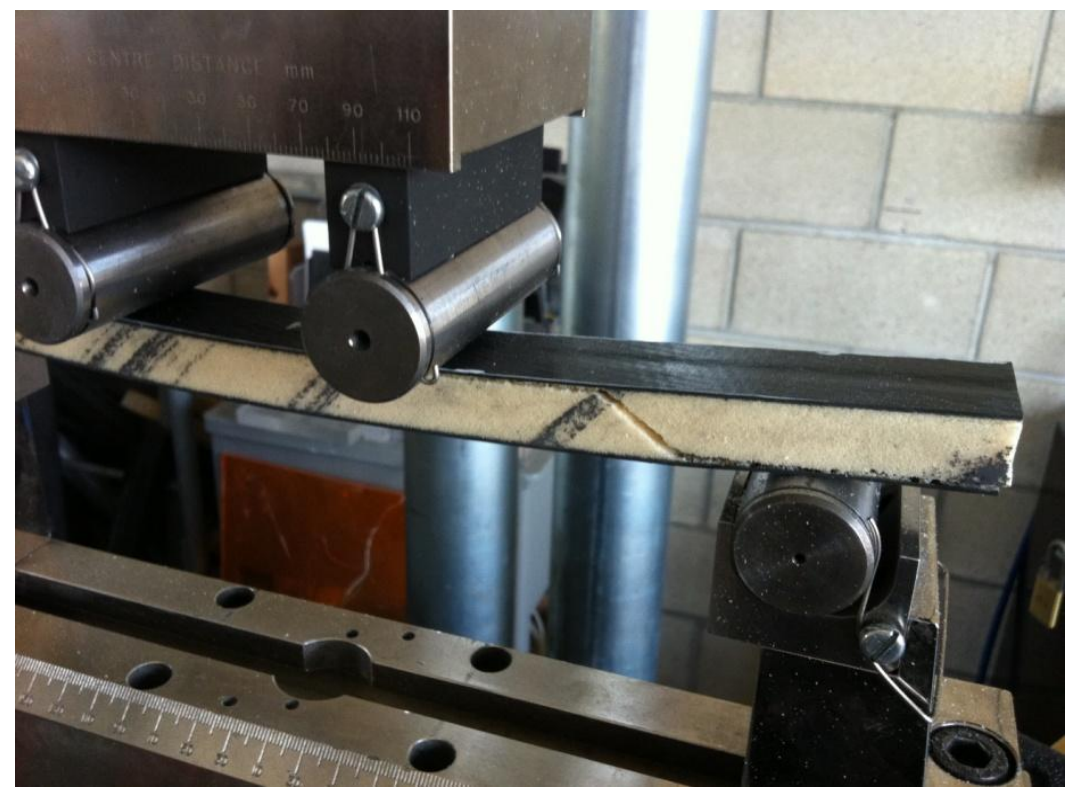

Figure 4.33. Typical failure seen in fatigue test at $1 \mathrm{~Hz}$ in beams with no initial delamination and longitudinal DAD key.

Although the number of cycles to failure varied widely across the group with added longitudinal keys, the failure mode observed was quite similar. The typical failure during 4-point 
bend fatigue testing can be seen in fig. 4.33. Similar to the control case, the failure mode observed began with a face-core interface failure and foam fracture. However, in this case the DAD key delayed fracture through the core. The face-core delamination propagated to the right of the left loaded cylinder, with fracture occurring two inches from right loaded cylinder. As depicted in fig. 4.33, the DAD key arrested fracture through the foam core to the right of the right loaded cylinder and face-core delamination continued past the right loaded cylinder and further propagated between the upper face sheet and core. Contrary to the previous two cases, some of the test specimens did fail due to fracture of the top face sheet. This indicates that the flexural strength of the sandwich beam was driven by the flexural strength of the face sheet and shear strength of the face-core bond. Figure 4.34 depicts the similar failure mode observed throughout the fatigue testing of the control case with an added longitudinal key.

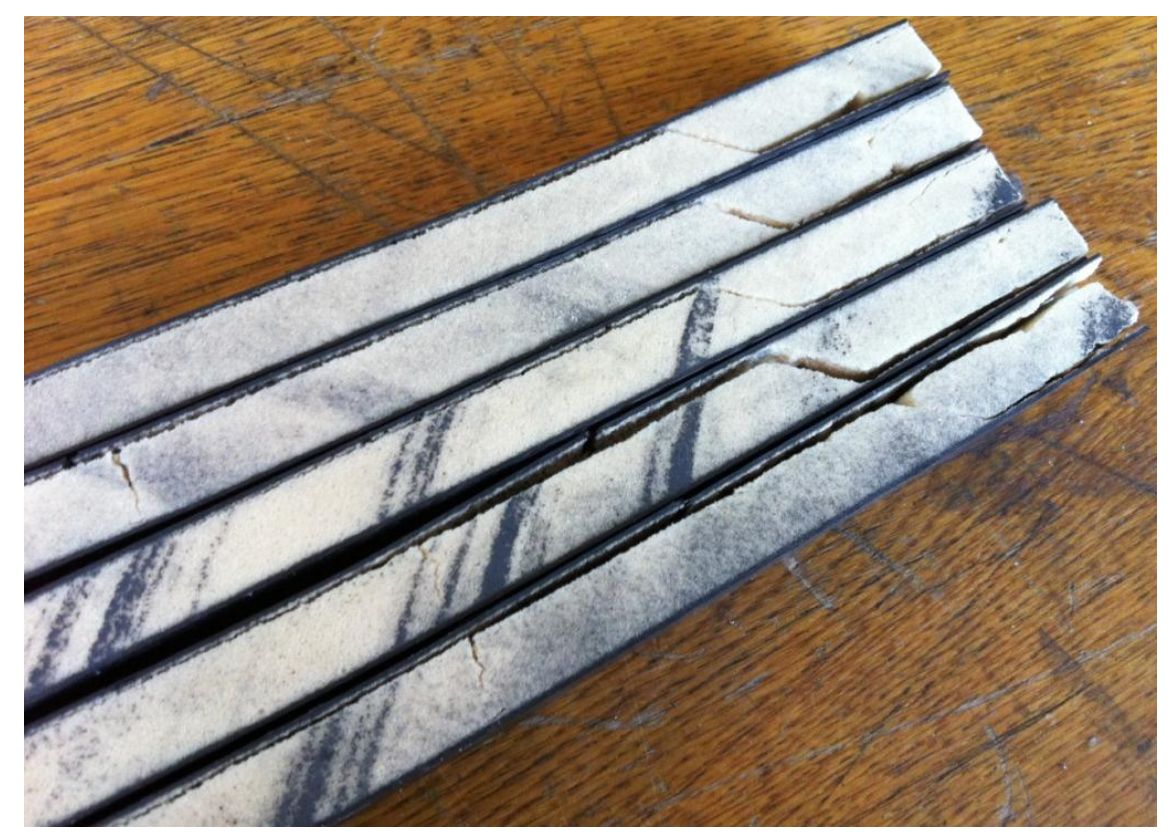

Figure 4.34. Test group with the addition of a longitudinal DAD key after final failure and completed fatigue life.

After testing the group with longitudinal DAD keys and no initial delamination under only 4-point bend fatigue test conditions, the beams which were previously impacted were tested. Again, to ensure that no creep or relaxation effects due to impact would occur, the beams were tested under fatigued under 4-point bend loading immediately following the impact testing. Five impacted test specimens with added longitudinal DAD keys were tested under the same fatigue test conditions as the non-impacted test specimens. The test specimens were tested to a mean 
load of $65 \%$ of the ultimate load of the impacted control test specimen and amplitude of $40 \%$ of the mean load. These values were determined to be $72 \mathrm{lbs}$ and $30 \mathrm{lbs}$, respectively. The results from the fatigue tests performed on the impacted control test specimen beams with the addition of longitudinal DAD keys are shown below in table 4.8. It can be seen that the average number of cycles to failure was 46,782 cycles with a percent error of $61.26 \%$. The percent error was high due to a premature failure seen in one test specimen and a very high fatigue life seen in another test specimen. Overall, with the same mean load value and amplitude as the impacted control case, it can be concluded that the average number of cycles to failure greatly increased with the addition of the longitudinal DAD keys. On average, the addition of the longitudinal DAD keys increased the number of cycles to failure by $1289.01 \%$. Again, any differences in fatigue life in this particular test group are generally due to inconsistent impact penetrations and uncontrollable microscopic defects of different sizes.

Table 4.8. Fatigue test results of impacted test beams with Longitudinal DAD keys using 100kN load cell.

\begin{tabular}{|c|c|c|}
\hline Test Specimen & $\mathrm{N}_{\mathrm{f}}$ (Cycles to Failure) & $\% \mathrm{~F}_{\text {ult }}$ \\
\hline 1 & 48,802 & $65 \%$ \\
\hline 2 & 79,829 & $65 \%$ \\
\hline 3 & 52,864 & $65 \%$ \\
\hline 4 & 654 & $65 \%$ \\
\hline 5 & 51,761 & $65 \%$ \\
\hline \multirow{2}{*}{ With Outliers } & Average $\mathrm{N}_{\mathrm{f}}$ & 46,782 \\
\cline { 2 - 3 } & Percent Error & $61.26 \%$ \\
\hline \multirow{2}{*}{ Without Outliers } & Average $\mathrm{N}_{\mathrm{f}}$ & 51,142 \\
\cline { 2 - 3 } & Percent Error & $4.12 \%$ \\
& & \\
& &
\end{tabular}




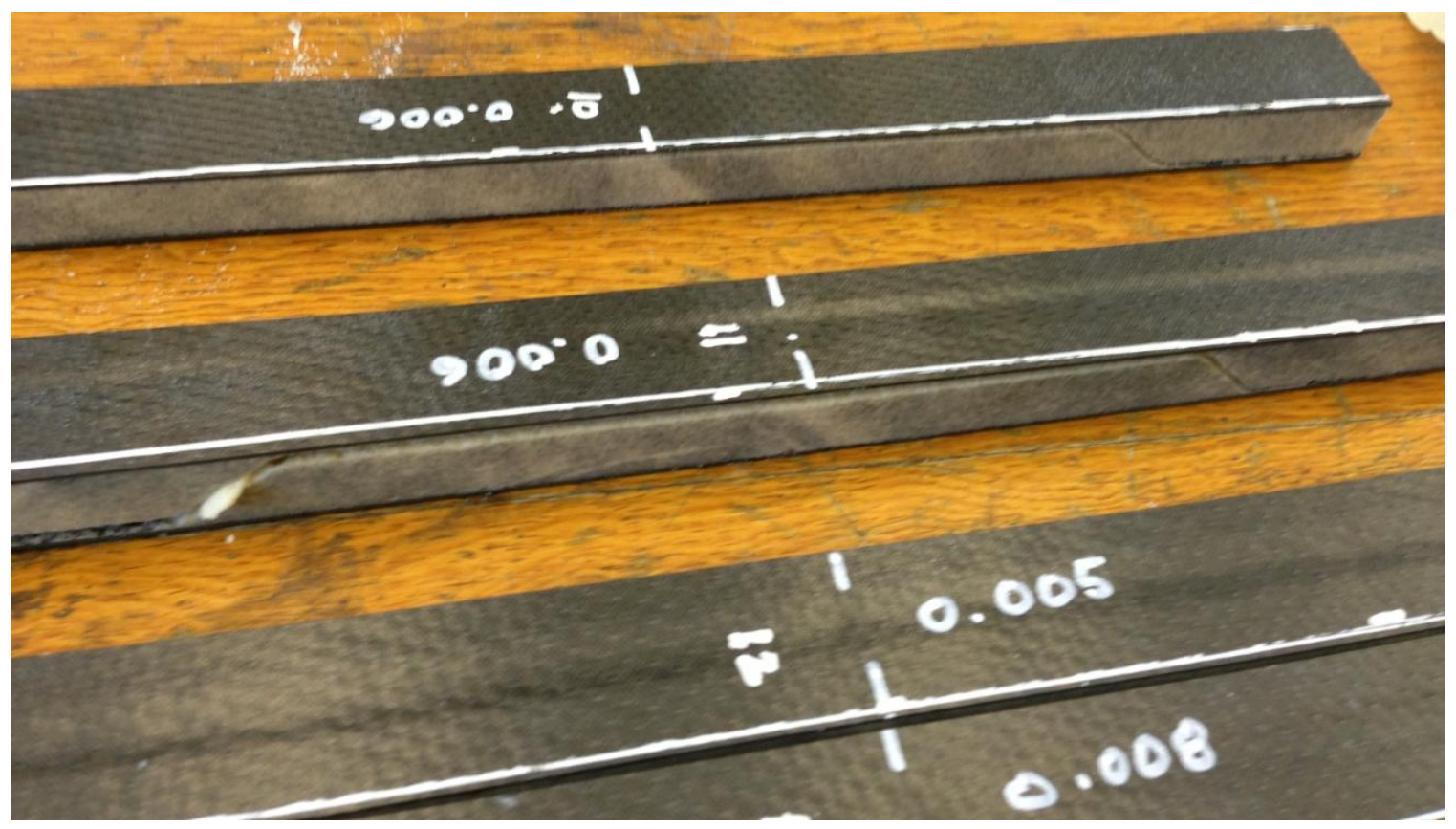

Figure 4.35. Typical failure from fatigue test at $1 \mathrm{~Hz}$ on impacted beam with no initial delamination and longitudinal DAD key.

The failure mode observed in the impacted case with no initial delamination and longitudinal DAD keys involved the same face-core failure and foam core fracture as the case with no initial delamination and longitudinal DAD keys, except that the core fracture occurred on both sides of the test beams. Figure 4.35 depicts the failure seen in the impacted control beam with no initial delamination and added longitudinal DAD keys after fatigue testing. This case was very different in the previous impacted cases because there was no visible failure seen in the top face sheet. Figure 4.36 shows that the only damage seen in the test specimens is due to the initial impact, where there is no matrix or fiber cracking. The ultimate failure seen in this test case indicates that the flexural strength of the beam was based largely upon the flexural strength of the foam core. 


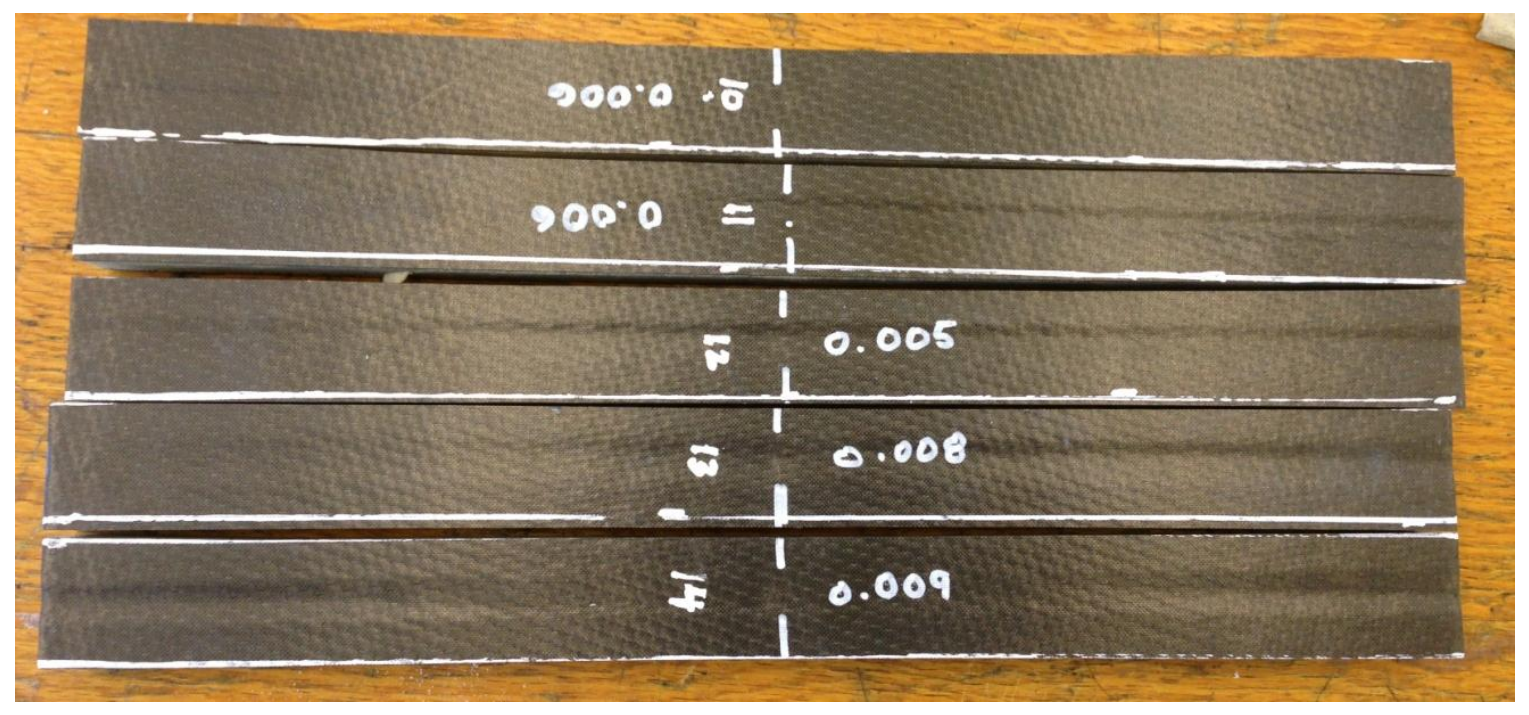

Figure 4.36. Impacted beams with added Longitudinal DAD keys after final failure and completed fatigue life.

\subsubsection{Initially Delaminated Beam}

The initially delaminated beams without DAD keys were tested under 4-point bend fatigue testing conditions at a $1 \mathrm{~Hz}$ frequency. In total, five specimens were tested to a mean load of $75 \%$ of the ultimate load of the initially delaminated beam without DAD keys and amplitude of $40 \%$ of the mean load. These values were determined to be $77 \mathrm{lbs}$ and $30 \mathrm{lbs}$, respectively. The mean load percentage was increased due to time constraints, since the delaminated case did not fail after 20,000 cycles with a mean load of $65 \%$ ultimate load of the specimen. The results from the fatigue tests performed on the test specimen beams with a one inch initial delamination are shown below in table 4.9. The average number of cycles to failure was 1,459 cycles with a percent error of $11.68 \%$, excluding the outlier values. The percent error including the outlier value is very high due to varying life cycles, with 165 cycles being the shortest fatigue life and 4,090 being the highest. This great variance can be due to the variance between each test specimen in the group and the placement of the test specimen in the 4-point bend jig. If the specimen is not placed directly in the center, the weight of the loaded cylinder can have a great effect, especially in the forced delamination case. 
Table 4.9. Fatigue test results of test beams with one inch initial delamination.

\begin{tabular}{|c|c|c|}
\hline Test Specimen & $\mathrm{N}_{\mathrm{f}}($ Cycles to Failure $)$ & $\% F_{\text {ult }}$ \\
\hline 1 & 165 & $75 \%$ \\
\hline 2 & 1,579 & $75 \%$ \\
\hline 3 & 4,090 & $75 \%$ \\
\hline 4 & 125 & $75 \%$ \\
\hline 5 & 1,338 & $75 \%$ \\
\hline \multirow[t]{2}{*}{ With Outliers } & Average $\mathrm{N}_{\mathrm{f}}$ & 1,459 \\
\hline & Percent Error & $110.51 \%$ \\
\hline \multirow[t]{2}{*}{ Without Outliers } & Average $N_{f}$ & 1,459 \\
\hline & Percent Error & $11.68 \%$ \\
\hline
\end{tabular}

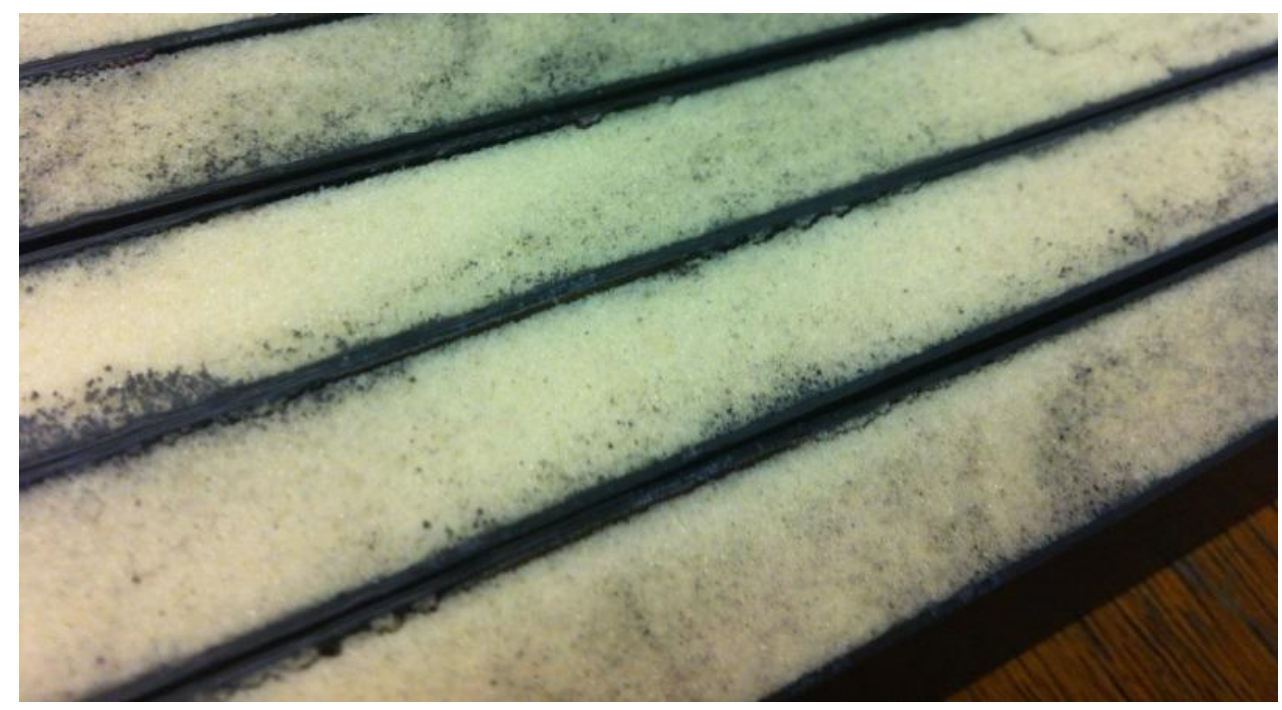

Figure 4.37. Typical failure from fatigue test at $1 \mathrm{~Hz}$ on beams with an initial delamination.

The variance in fatigue life values in the group with a forced 1-inch delamination is very high, but each test specimen failed in the same manner. The typical failure during 4-point bend fatigue testing can be seen in fig. 4.37. As shown above, initial failure occurred due to face-core delamination in between the load span in all test cases. The initially delaminated beams failed similarly to the static 4-point bend case, where there was an evident face-core separation under the load span area that continued one inch past the left loaded cylinder and one inch past the 
right loaded cylinder. The delamination in this case is very symmetrical and continues about one inch past the initial delamination in both directions, as seen in fig. 4.37. In this case, there is no complete failure through the core to the other face sheet, as seen in the static 4-point bend test. No visible failure to the composite face sheet was observed, which indicates that the flexural strength of this case was largely driven by the flexural strength of the foam core.

\subsubsection{Initially Delaminated Beam with Transverse DADs}

The initially delaminated beams with added transverse DAD keys were tested under 4point bend fatigue test conditions at a $1 \mathrm{~Hz}$ frequency. The objective of adding the transversely oriented DAD keys in the delaminated test specimen was to see if an increase in fatigue life occurred. Therefore, five specimens were tested to a mean load of $75 \%$ of the ultimate load of the initially delaminated beam and amplitude of $40 \%$ of the mean load. These values were determined to be $77 \mathrm{lbs}$ and $30 \mathrm{lbs}$, respectively. The results from the fatigue tests performed on the test specimen beams with a one inch initial delamination and added transverse DAD keys are shown below in table 4.10. In this case, the test specimen beams did not fail after 50,000 cycles. In order to save time, fatigue testing ended when the number of cycles exceeded $30,000-50,000$ cycles. This was done because the increase in fatigue life proved to be significant enough to conclude that the DAD keys increased the number of cycles to failure significantly. 
Table 4.10. Fatigue test results of test beams with one inch initial delamination and transverse DAD keys.

\begin{tabular}{|c|l|c|}
\hline Test Specimen & $\mathrm{N}_{\mathrm{f}}$ (Cycles to Failure) & \% $\mathrm{F}_{\text {ult }}$ \\
\hline 1 & $>33,684$ & $75 \%$ \\
\hline 2 & $>33,396$ & $75 \%$ \\
\hline 3 & $>30,078$ & $75 \%$ \\
\hline 4 & $>50,001$ & $75 \%$ \\
\hline 5 & 3,244 & \\
\hline \multirow{2}{*}{ With Outliers } & Average $\mathrm{N}_{\mathrm{f}}$ & \\
\cline { 2 - 3 } & Percent Error & \\
\hline \multirow{2}{*}{ Without Outliers } & Average $\mathrm{N}_{\mathrm{f}}$ & \\
\cline { 2 - 3 } & Percent Error & \\
\hline
\end{tabular}

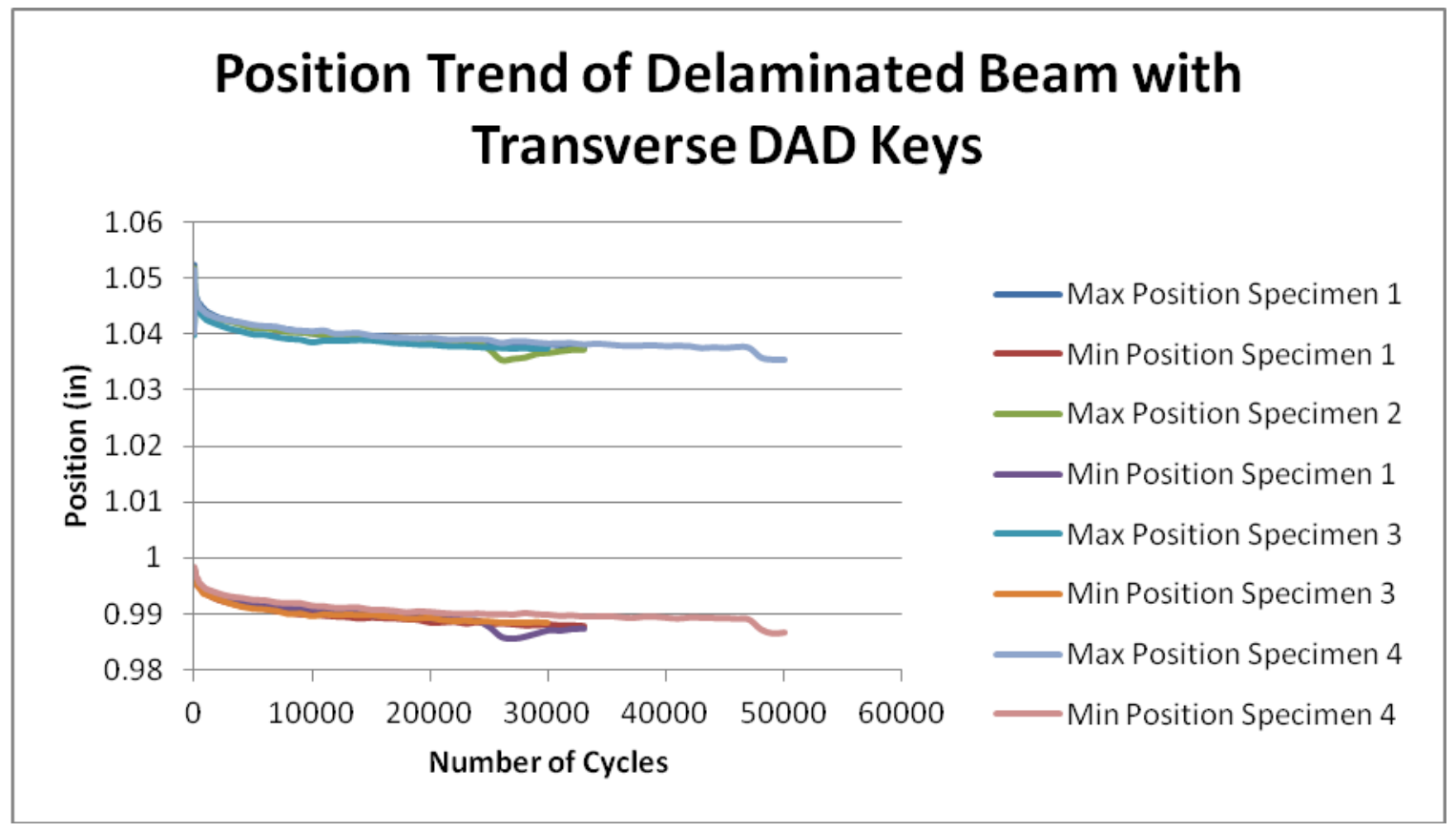

Figure 4.38. Position trend of delaminated beam with transverse DAD keys. 


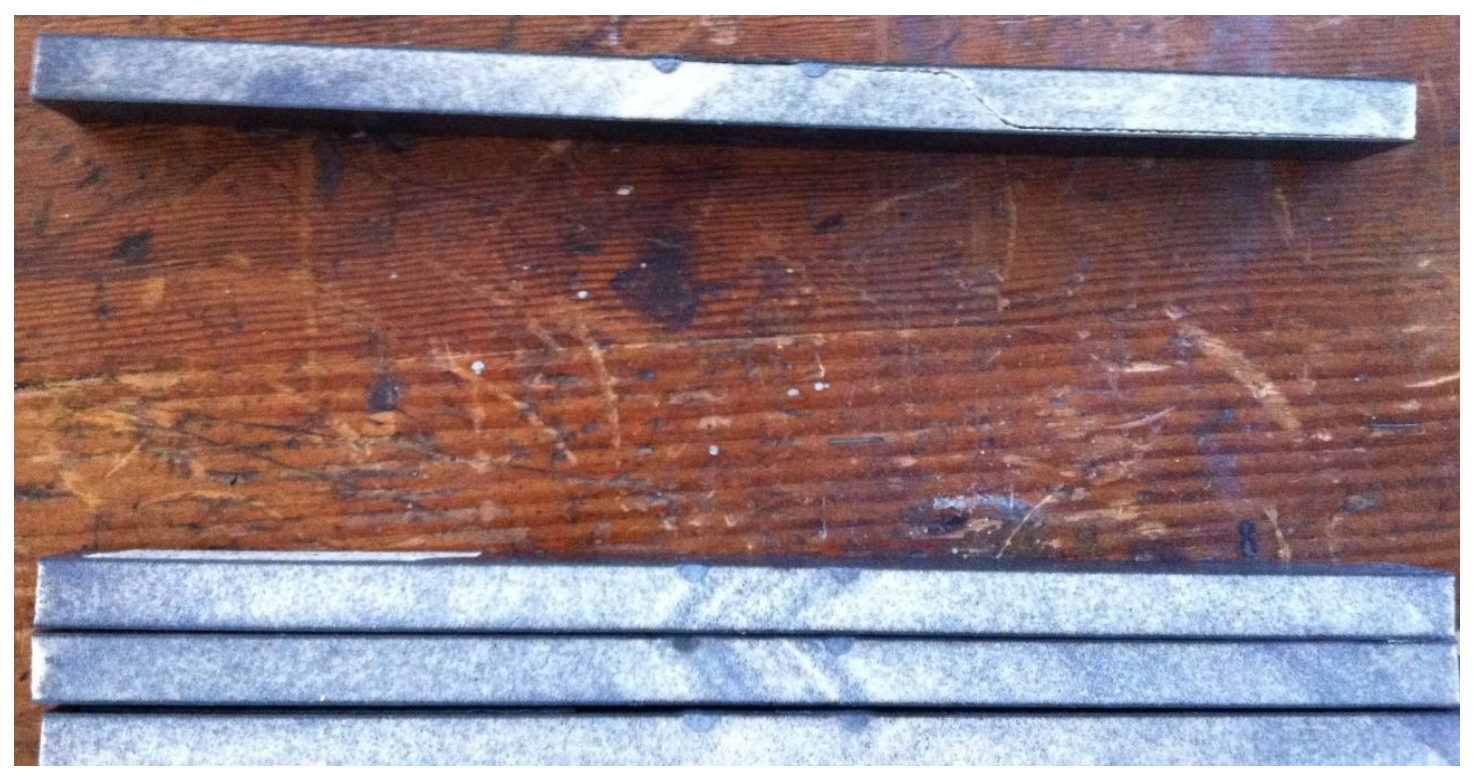

Figure 4.39. premature failure from fatigue test at $1 \mathrm{~Hz}$ on control beam with initial delamination and transverse DAD key.

The addition of transverse DAD keys proved to provide the necessary stiffness to extend the fatigue life of the delaminated test beams. As shown in table 4.10, the fatigue life of the test beams had the capability of exceeding 50,000 cycles. In order to show that the test specimens had the capability of exceeding 50,000 cycles, a position trend of the test specimens that did not fail is shown in fig. 4.38. It can be seen that the position trend of the test beam at 50,000 cycles does not change very much from the position of the test specimens at $\sim 30,000$ cycles. Therefore, the assumption that all test specimens could exceed 50,000 cycles is very plausible. Although most test specimens performed very well, there was one test specimen that failed prematurely during the 4-point bend fatigue test and this can be due to many errors such as; placement of test specimen in 4-point bend fixture, manufacturing flaw, etc. There was an evident face-core separation under the load span area that continued one inch past the right loaded cylinder. In this case, final failure occurs through a shear in the core to the other face sheet, where there is also face-core separation in the bottom face sheet, as shown in fig. 4.39. No visible failure to the composite face sheet was observed, which indicates that the flexural strength of the initially delaminated beams with added transverse DAD keys was largely driven by the flexural strength of the foam core. 


\subsubsection{Initially Delaminated Beam with Longitudinal DAD}

The initially delaminated beam case with a longitudinal DAD key was the last case tested under fatigue loading at a $1 \mathrm{~Hz}$ frequency. The objective of adding the longitudinally oriented DAD key in the delaminated control specimen was to see if the increase in stiffness would in turn increase the fatigue life. Therefore, five specimens were tested to a mean load of $75 \%$ of the ultimate load of the initially delaminated beam case without DAD keys and amplitude of $40 \%$ of the mean load. These values were determined to be $77 \mathrm{lbs}$ and $30 \mathrm{lbs}$, respectively. The results from the fatigue tests performed on the initially delaminated beams with an added longitudinal DAD key are shown below in table 4.11. In this case, the test specimen beams did not fail after 45,000 cycles. In order to save time, fatigue testing ended when the number of cycles exceeded $35,000-45,000$ cycles. Similar to the previous case; this was done because the increase in fatigue life proved to be significant enough to conclude that the longitudinal DAD key increased the number of cycles to failure significantly.

Table 4.11. Fatigue test results of test beams with one inch initial delamination and Longitudinal DAD key.

\begin{tabular}{|c|l|c|}
\hline Test Specimen & $\mathrm{N}_{\mathrm{f}}$ (Cycles to Failure) & \% $\mathrm{F}_{\text {ult }}$ \\
\hline 1 & $>36,764$ & $75 \%$ \\
\hline 2 & $>45,833$ & $75 \%$ \\
\hline 3 & 384 & $75 \%$ \\
\hline 4 & $>35,208$ & $75 \%$ \\
\hline 5 & $>37,114$ & \\
\hline \multirow{2}{*}{ With Outliers } & Average $\mathrm{N}_{\mathrm{f}}$ & \\
\cline { 2 - 3 } & Percent Error & \\
\hline \multirow{2}{*}{ Without Outliers } & Average $\mathrm{N}_{\mathrm{f}}$ & \\
\cline { 2 - 3 } & Percent Error & \\
\hline
\end{tabular}




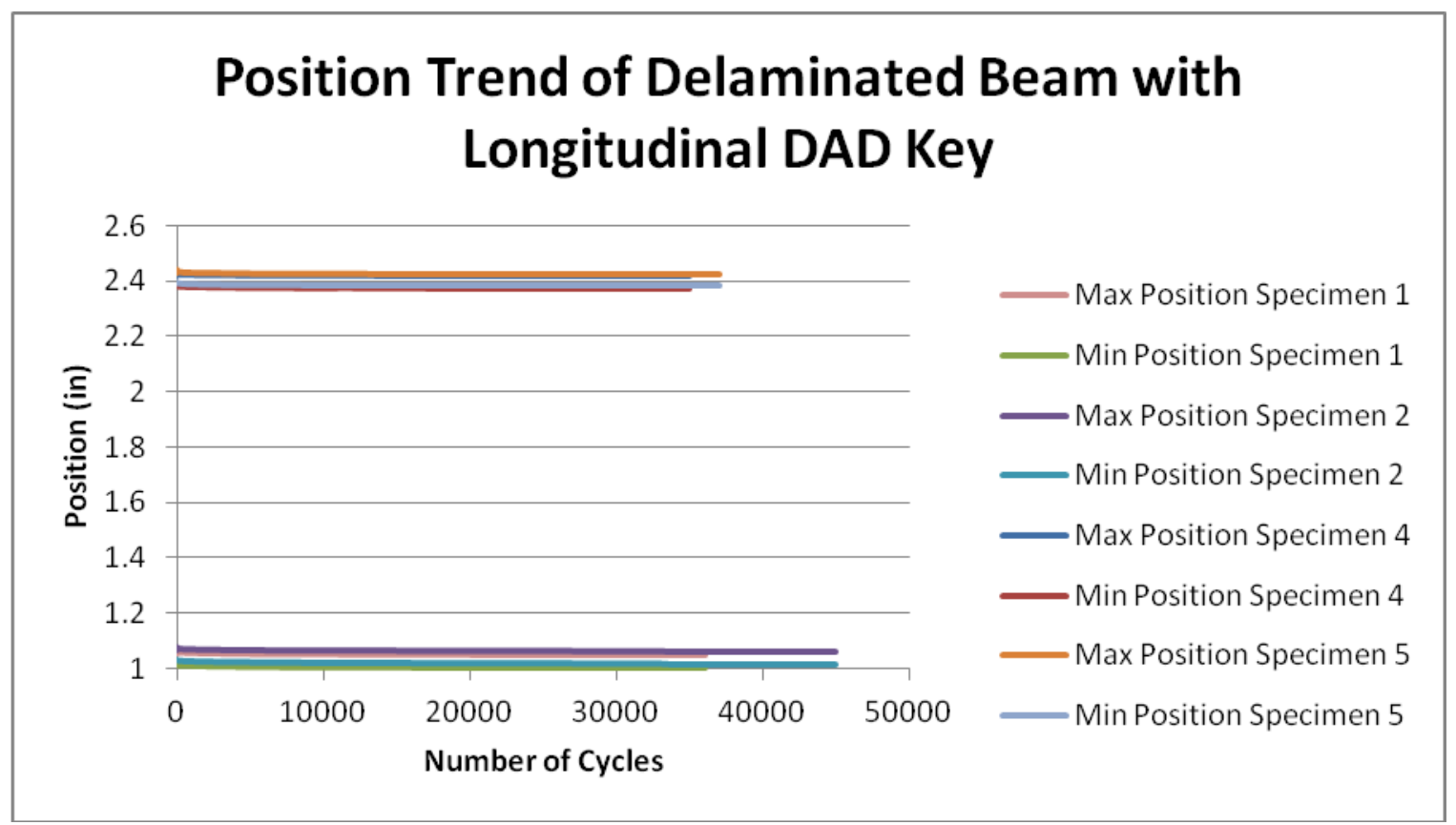

Figure 4.40. Position trend of delaminated beam with Longitudinal DAD key.

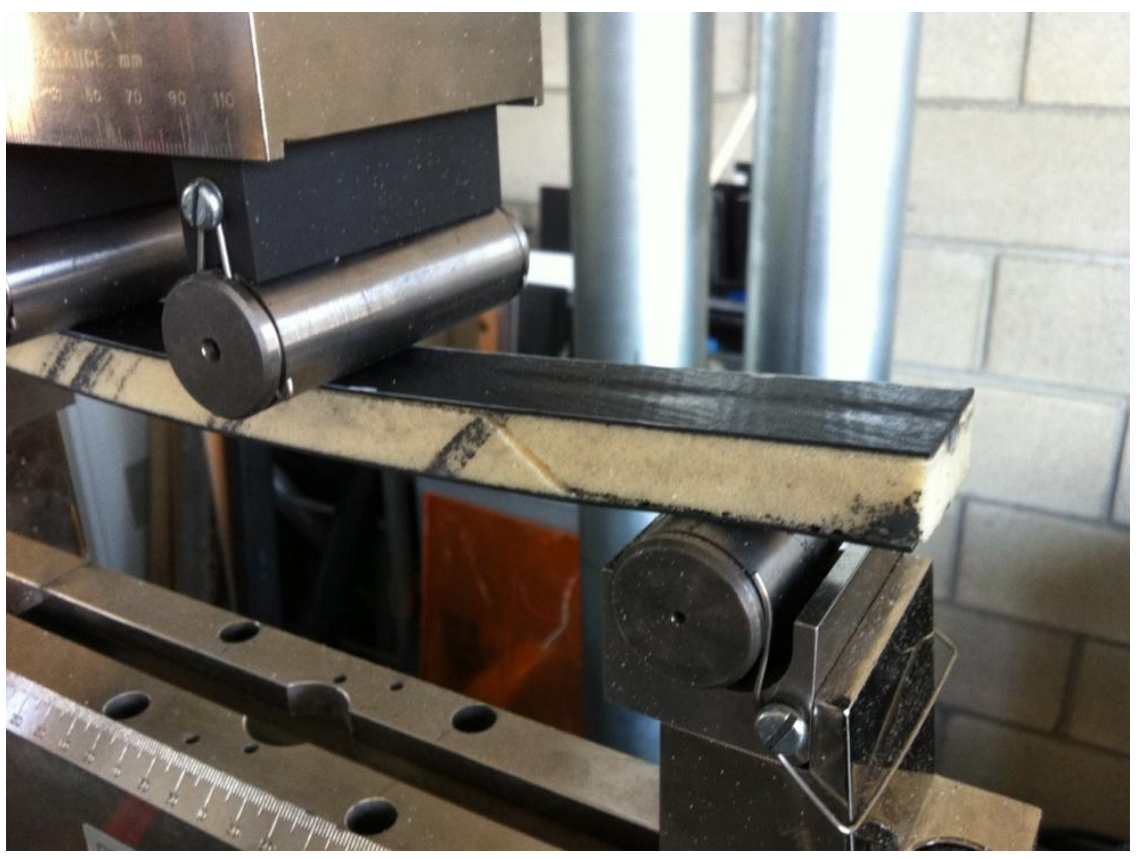

Figure 4.41. Premature failure seen from fatigue test at $1 \mathrm{~Hz}$ on beam with initial delamination and longitudinal DAD key.

The longitudinal DAD keys proved to extend the fatigue life of the beam with a 1 -inch delamination. Through 4-point bend fatigue testing, the beams with an initial delamination and an 
added longitudinal DAD key showed no sign of failure. As shown in table 4.11, the fatigue life of the test beams had the capability of exceeding 45,000 cycles. In order to show that the test specimens had the capability of exceeding 45,000 cycles, a position trend of the test specimens that did not fail is shown in fig. 4.40. It can be seen that the position trend of the test beam at 45,000 cycles does not change very much from the position of the test specimens at $\sim 35,000$ cycles. Similar to the previous case, the assumption that all test specimens could exceed 45,000 cycles is very reasonable. One test specimen failed prematurely during the 4-point bend fatigue test. As shown in fig. 4.41, face-core separation occurred under the load span area and continued one inch past the right loaded cylinder. Final failure occurred through a shear in the core to the other face sheet, where face-core separation continued in the bottom face sheet. Again, no visible failure to the composite face sheet was observed, which indicates that the flexural strength of the case with an initial one inch delamination and added longitudinal DAD key was largely driven by the flexural strength of the foam core. 


\section{Chapter 5 Finite Element Analysis}

Finite element analysis (FEA) was performed on six different composite sandwich beam configurations constructed of carbon composite face sheets, PVC foam core, and fiberglass damage arrestment devices (DADs). Six cases were modeled; a non-delaminated beam and an initially delaminated beam, each with the addition of transversely placed DADs and a longitudinally placed DAD. Each structure was subjected to a four-point bending condition with two 50-lb forces acting on the upper face sheets of the beam. The foam core and fiberglass DADs were both modeled as solid elements, while the face sheets were modeled as shell elements. A convergence study was performed and a seed size of 0.1 " was used for all analysis. Under the consistent loading condition, the non-delaminated structure was found to be insignificantly affected by the addition of either DAD configuration and only decreased the deflection by approximately 0.06 percent. The delaminated structure was significantly affected by the addition of the DADs, where the DADs caused a decrease in deflection of up to 6.3 percent. The deflections for the various DAD key configurations found in the experimental work were in agreement with the finite element results and differed between 1-14 percent.

\subsection{Model Development}

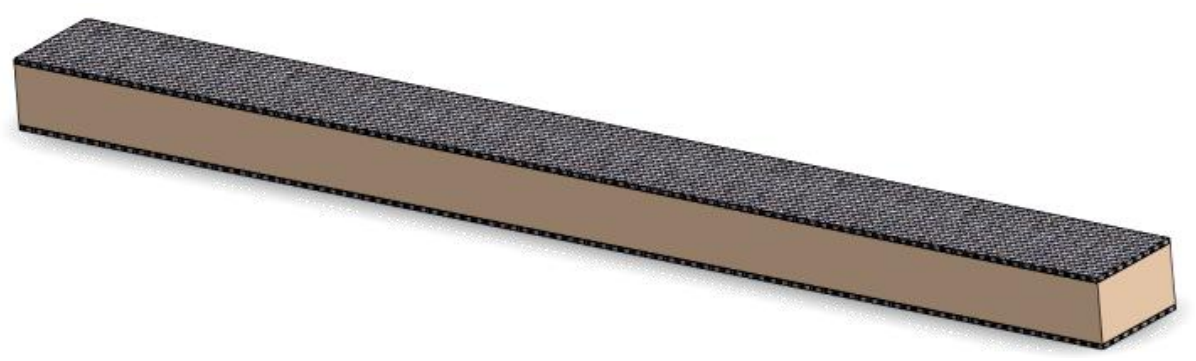

Figure 5.1. Composite Sandwich control Beam model.

Abaqus, developed by Dassault Systemes, was the finite element modeling software of choice in this research. Abaqus was used to model six configurations of the composite sandwich beam, all under 4-point loading. Six configurations of the composite sandwich beam are 
considered. Two control cases are considered, a non-delaminated beam and a delaminated beam. For the delaminated case, an initial delamination is introduced in between the load span of the beam and is one inch in length. Each of these cases consists of two structure-critical parts that were modeled in Abaqus.

- $\quad$ Face Sheets (2)

- Core (1)

The non-delaminated and delaminated beam cases are then tested with the addition of DADs and each of these configurations consists of three structure-critical parts that were modeled in Abaqus.

- $\quad$ Face Sheets (2)

- Core (1)

- $\operatorname{DADs}(1,2)$

Each of the structural components were considered and modeled in Abaqus. As shown in the non-delaminated beam with transverse DADs in fig. 5.2 and the non-delaminated beam with one longitudinal DAD in fig. 5.3; the face sheets are shown in black, the core is shown in tan, and the DADs in white.

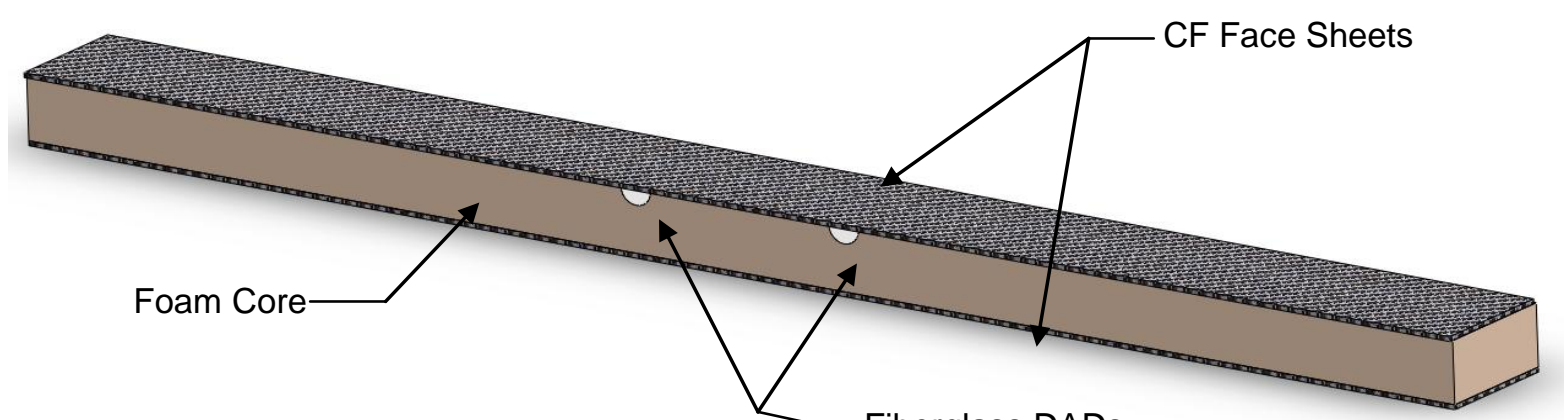

Figure 5.2. Non-Delaminated Beam with Firanglass. DADS

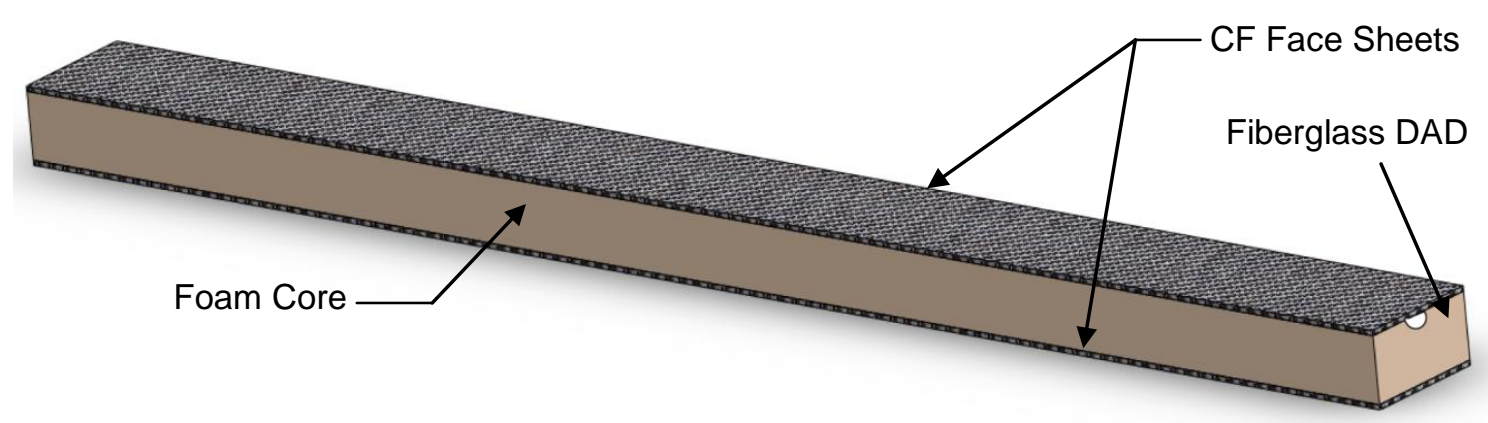

Figure 5.3. Non-Delaminated Beam with Longitudinal DAD. 


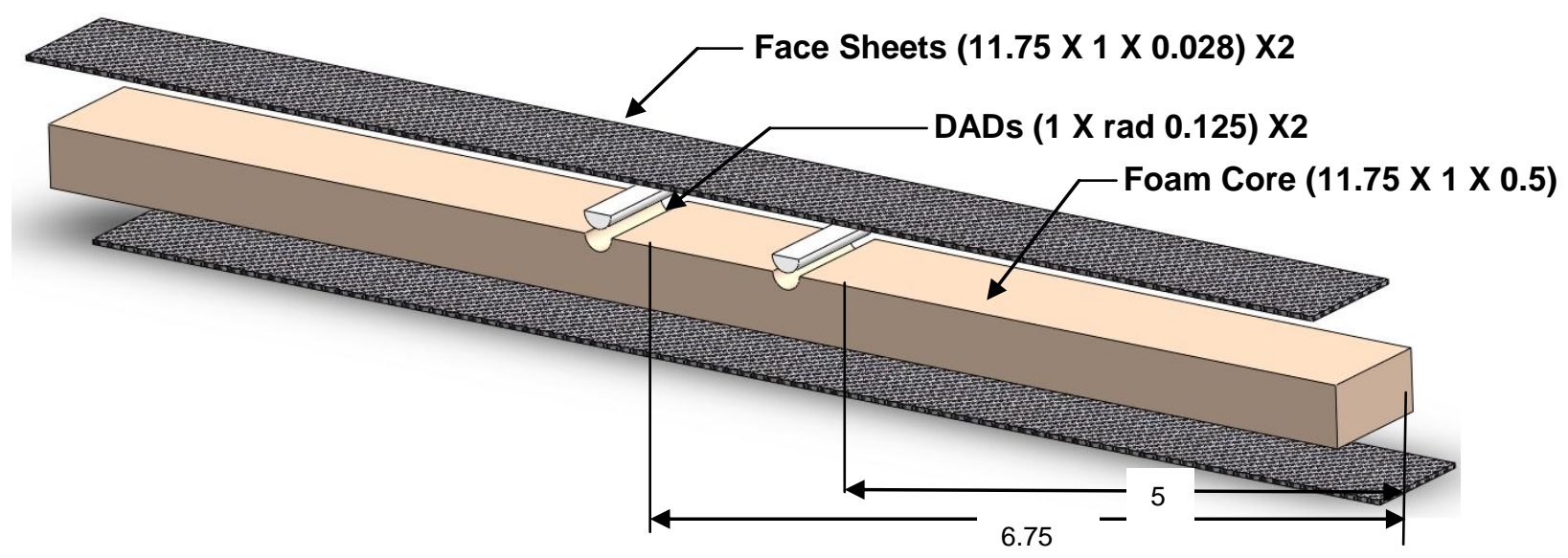

Figure 5.4. Non-Delaminated Sandwich Beam with transverse DADs Exproaed View (inches).

Abaqus was used to model a representation of the composite sandwich beam for each of the six configurations. Each part was given an elastic definition, density, and dimension as shown in Table 5.1. The material properties shown were found through the experimental portion of this research as discussed in Section 2. Each component in the model was defined in the base length unit of inches.

Table. 5.1. FE Simulation material properties for composite sandwich beam.

\begin{tabular}{|c|c|c|c|c|c|c|c|c|c|}
\hline Part & Material & $E(p s i)$ & $\begin{array}{l}\text { Poisson's } \\
\text { Ratio }\end{array}$ & $\begin{array}{l}\text { Length } \\
\text { (in) }\end{array}$ & $\begin{array}{l}\text { Width } \\
\text { (in) }\end{array}$ & $\begin{array}{l}\text { Depth } \\
\text { (in) }\end{array}$ & $\begin{array}{l}\text { Radius } \\
\text { (in) }\end{array}$ & $\begin{array}{l}\text { Part } \\
\text { Type }\end{array}$ & $\begin{array}{l}\text { Section } \\
\text { Type }\end{array}$ \\
\hline Core & $\begin{array}{l}\text { Last-A-Foam } \\
\text { FR } 6710 \text { PVC } \\
\text { Foam }\end{array}$ & 7,700 & 0.31 & 11.75 & 1 & 0.5 & - & $\begin{array}{l}\text { 3-D } \\
\text { Solid }\end{array}$ & $\begin{array}{l}\text { 3-D } \\
\text { Solid }\end{array}$ \\
\hline $\begin{array}{l}\text { Face } \\
\text { Sheets }\end{array}$ & $\begin{array}{l}\text { LTM45/CF1803 } \\
\text { Carbon Fiber }\end{array}$ & $9,619,014$ & 0.096 & 11.75 & 1 & 0.0385 & - & $\begin{array}{l}\text { 3-D } \\
\text { Shell }\end{array}$ & $\begin{array}{l}-D \\
\text { Shell }\end{array}$ \\
\hline DAD & $\begin{array}{l}\text { Woven } \\
\text { Roving Fiberglass }\end{array}$ & $3,304,000$ & 0.26 & 0.25 & 1 & - & 0.125 & $\begin{array}{l}\text { 3-D } \\
\text { Solid }\end{array}$ & $\begin{array}{l}\text { 3-D } \\
\text { Solid }\end{array}$ \\
\hline
\end{tabular}

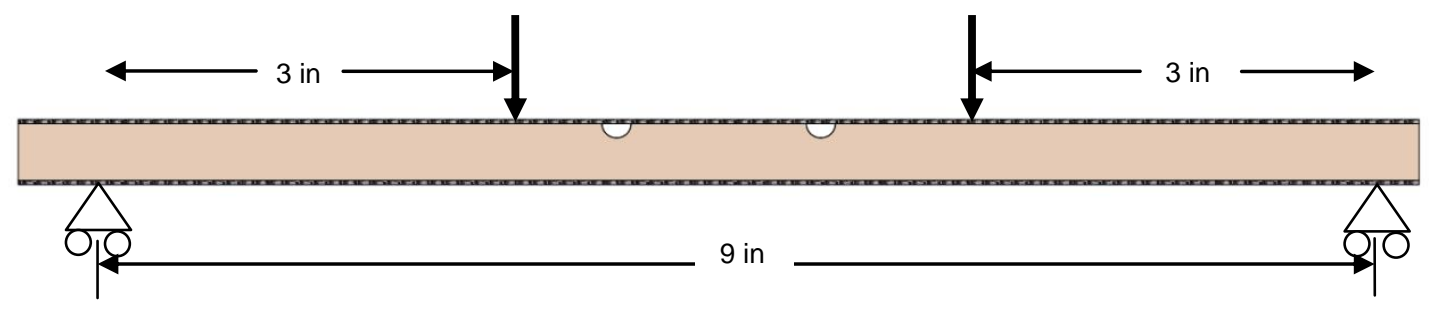

Figure 5.5. Four-Point Bending Loading Condition, Two 50-lb loads.

The standard units of the output used force in pounds, due to the inch units used, but pressures were measured in pounds per square inch (psi). The composite sandwich beam with no delamination was modeled as two separate parts in Abaqus. The first part was the PVC foam core, which was modeled as a 3-D deformable solid. The second part was the carbon fiber face 
sheets, which were modeled as a 3-D shell. A solid section was created with the PVC foam material properties shown in Table 5.1 for implementation in the core. Similarly, a shell section was created for implementation in the face sheets, with the given material properties of the carbon fiber used and a shell thickness corresponding to the face sheet depth. The two face sheets were sketched and modeled as one part; therefore the distance between the two face sheets accounted for both the thickness of the core and the thickness of one face sheet since Abaqus generates the shell thickness equally between the sketch lines. In the assembly, the instances were translated to their appropriate places before each part received their appropriate constraints. Since the bonding strength from the sheet resin between the carbon fiber and the foam is unknown, two tie constraints were created in order to allow for shared nodes between the two parts. For the first tie constraint, the top surface of the core was chosen as the master and the bottom surface of the top face sheet was chosen as the slave. The second tie constraint consisted of a tie between the bottom surface of the core and the top surface of the bottom face sheet. The surface of the core was chosen as the slave surface, while the surface of the bottom face sheet was chosen as the master.

The composite sandwich beam with an initial delamination was modeled identically with the exception of the tie constraints. In order to model the delamination, the tie constraint must be modified slightly to account for the one inch delamination in the center of the beam span. First a partition was made to the bottom face of the top face sheet. This partition created a one inch square located in the center span of the face sheet. Once this was completed, the tie constraint the ties the top face sheet to the core was modified to exclude the one inch partitioned square by selecting all surfaces of the top face sheet except the one inch square in the center. This ensures that the middle square and the foam core do not share nodes, and therefore simulate a delamination at the face core interface. The second tie constraint is constrained identically to the non-delaminated control case.

The composite sandwich beams with no delamination and the addition of the DADs were modeled as three separate parts in Abaqus. The first part was the PVC foam core, which was modeled as a 3-D deformable solid. The second part was the carbon fiber face sheets, which 
were modeled as a 3-D shell. The third part was the fiberglass DAD, which was modeled as a 3-D solid. The sections for the face sheets and core were modeled similarly to the previous models; however, the third section created was a 3-D solid section with the material properties of the fiberglass seen in table 5.1. This section was assigned to the fiberglass parts in the model, which totaled to two for the transverse DAD key case and one for the longitudinal DAD key case. In the assembly, the instances were translated to their appropriate places before each part received their appropriate constraints. Three tie constraints were created in order to allow for shared nodes between the three parts. For the first tie constraint, the top surface of the core and the top surfaces of the DADs were chosen as the master and the bottom surface of the top face sheet was chosen as the slave. The second tie constraint consisted of a tie between the bottom surface of the core and the top surface of the bottom face sheet. The surface of the core was chosen as the slave surface, while the surface of the bottom face sheet was chosen as the master. The last tie constraint tied the DADs to the foam core. The bottom surfaces of the DADs were chosen as the slave and the top face of the foam core was chosen as the master.

The final beam configurations consisted of models that included both initial delamination and DADs. These models were modeled similar to the composite sandwich beams with no delamination and the addition of DADs with the exception of the tie constraints. The delamination was again modeled similarly to the composite sandwich beam with delamination in that a partition was created in order to exclude the one inch square in the center of the beam span from the tie constraint between the face sheet and the core.

\subsection{Static Load Analysis}

To ensure the composite sandwich beam was modeled correctly, a model of a composite beam was created with the same static loading and boundary conditions as the sandwich beams tested in experiment. Using this method, the FE maximum deflection results could be compared with theoretical hand calculations found by analyzing the system in closed form. The model was made out of a layer of steel $\left(E_{S T}=30 \times 10^{6} \mathrm{psi}\right)$ sandwiched between two layers of aluminum $\left(E_{\mathrm{AL}}\right.$ $\left.=10 \times 10^{6} \mathrm{psi}\right)$. The loading condition consisted of two 50 -lb loads placed near the center span of the beam as shown in fig. 5.5. The total load of 100 pounds was chosen because it lies in the 
linear region of the load-deflection curve. Because the simulation assumes small angles of deflection and linearity, the load value of 100 pounds kept the response within that regime.

\subsubsection{Model Validity}

The static load case discussed was used to compare the FE results to the hand calculations. The method of equivalent stiffness's [29] was used to find the model's deflection theoretically. Because the elastic modulus of the steel is three times that of the aluminum, we can make one equivalent cross-section of aluminum by replacing the steel with aluminum that is three times as large, which can be seen in the appendix. The equation used to find the theoretical maximum deflection of the beam was found from a mechanics textbook for a simple four-point loading condition. By modeling the loading and boundary conditions correctly, the FE results yielded a deflection very close to the theoretical hand calculations and can be seen in table 5.2 .

Table 5.2. FE model and hand calculation comparison.

\begin{tabular}{|l|l|}
\hline Parameter & Result \\
\hline FE Max Deflection [in] & -0.00363 \\
\hline Theoretical Max Deflection [in] & -0.00348 \\
\hline
\end{tabular}

The results correspond very well, which shows that a FE model tested in experiment, consisting of the carbon fiber and PVC foam, should give acceptable results. The same loading condition was used to check for mesh convergence on the non-delaminated control beam. This was done to produce an adequate mesh to be used for the other configurations of the beam. Square linear elements were used since the geometry of the model was relatively simple and rectangular.

\subsection{Mesh Convergence}

To check mesh convergence, the left support restricted the model from moving in any of the three degrees of freedom $(\mathrm{U} 1=\mathrm{U} 2=\mathrm{u} 3=0)$. However, since the model was free to move in the direction parallel to the span of the beam, the right support only restricted the movement in the remaining two directions $(\mathrm{U} 2=\mathrm{U} 3=0)$. Only one support restricted the movement in all three 
degrees of freedom because without doing so, the system would become dynamic and the model would not yield accurate results. Two 50-lb loads were placed at the center of the beam span on the upper face sheet, as shown in fig. 5.5. The 50-lb loads were modeled as pressure loads with a total pressure of $100 \mathrm{psi}$ for each load since the applied area was $\mathrm{A}=0.05 \mathrm{in}^{2}$. This was done so that the numerical analysis reflected the same conditions as the four-point bending jig.

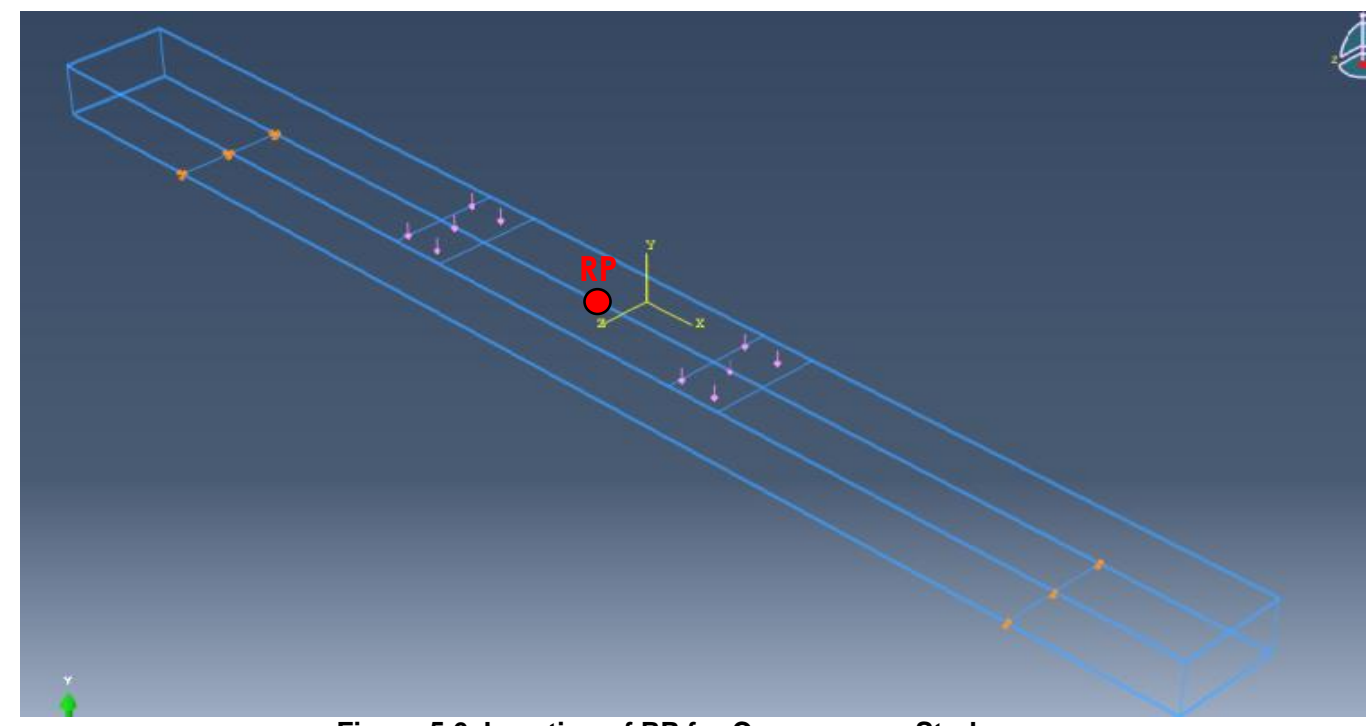

Figure 5.6. Location of RP for Convergence Study.

Two convergence studies were performed to check for mesh convergence. Maximum deflection and stress on the upper surface of the top face sheet, as shown in fig. 5.6, were used as mesh convergence parameters. The maximum deflection was taken from the mid-span of the beam, where the greatest displacement occurs. Figure 5.7 shows the convergence graph for maximum deflection at the mid-span, whereas fig. 5.8 shows the convergence graph for stress measurements taken at the RP. The number of degrees of freedom was varied by changing the seed size and in this study the seed size varied between 0.25 and 0.0625 in. As shown, both graphs begin to converge at a point corresponding to 70,482 degrees of freedom. This corresponds to a part seed size of $0.1 "$.

The refined mesh was seeded with a global size of 0.1 " for all parts in the model. For the 3-D solid element parts, linear elements were used with reduced integration turned off. For the 3D shell element parts, quadratic elements were used with reduced integration turned off. This was done so each element in the mesh was made up of an 8-noded element. Therefore, with a seed 
size of 0.1 ", the number of 8 -noded elements totaled to 8410 in the entire model. Thereafter the loading of two 50-lb loads, applied to the upper face sheet of the beam, was imposed on the model. This was modeled using Abaqus/Standard, similar to the loading of the validation model. This process was chosen since this was a static loading condition. After obtaining the maximum displacement values for the non-delaminated control beam using FE analysis, the remaining configurations were meshed identically. The elements found in these remaining configurations yielded very similar elements to the validation model.

The static model had a few considerable warnings, but they were negligible. The most significant set of warnings warned about certain nodes in specified surfaces of tie constraints not being tied. However, this was to be expected since there were some surfaces that were not tied purposely for the delaminated cases.

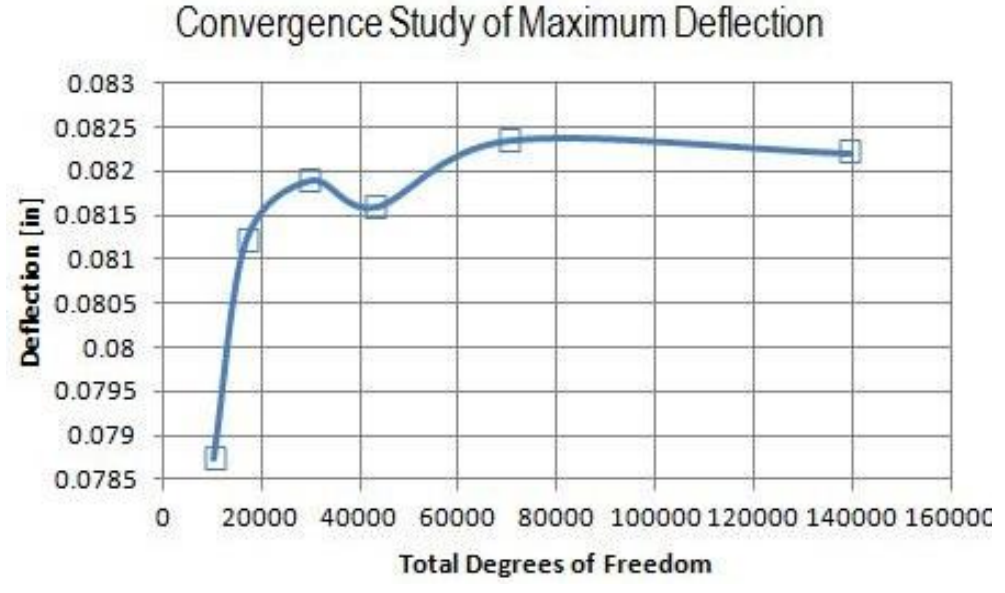

Figure 5.7. Convergence Study of Max Deflection.

Convergence Study of Stress atRP

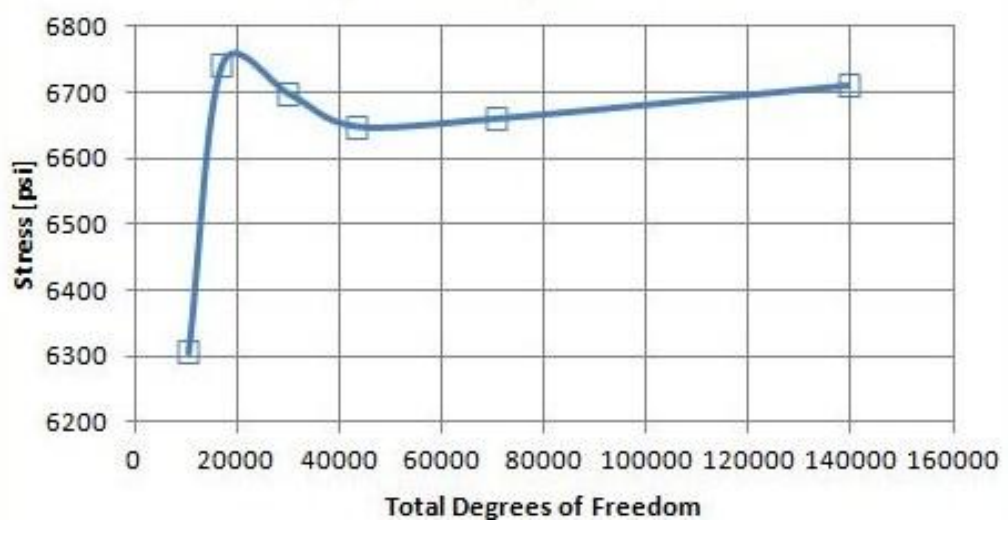

Figure 5.8. Convergence Study of Stress at RP. 


\subsection{Results}

The 4-point loading condition was applied to each of the six configurations of the model as shown in fig. 5.9. Since the validation model using different materials but similar loading conditions and boundary conditions was verified to be accurate, it can be assumed that the six configurations modeled are accurate despite the change in materials. Recall, the materials used for the validation were aluminum and steel and changed to the actual model's materials of carbon fiber, foam, and fiberglass.

The stress and displacements of the non-delaminated control beam under four-point bending can be found in table 5.3 below.

Table 5.3. Results for four-point bending condition on non-delaminated control beam.

\begin{tabular}{|l|l|}
\hline \multicolumn{2}{|l|}{ Mid Span Load $=2 \times 50 \mathrm{lbs}$} \\
\hline Max Deflection & $-0.1001 \mathrm{in}$ \\
\hline Max Deflection- Experimental & $-0.1014 \mathrm{in}$ \\
\hline Max Beam von Mises & $13.48 \mathrm{ksi}$ \\
\hline
\end{tabular}

The resulting deflection matches the experimental results within $1.3 \%$, indicating an acceptable model. As expected, the maximum normal stress is found in the supports. The beam deflection can be seen in fig. 5.9 below.

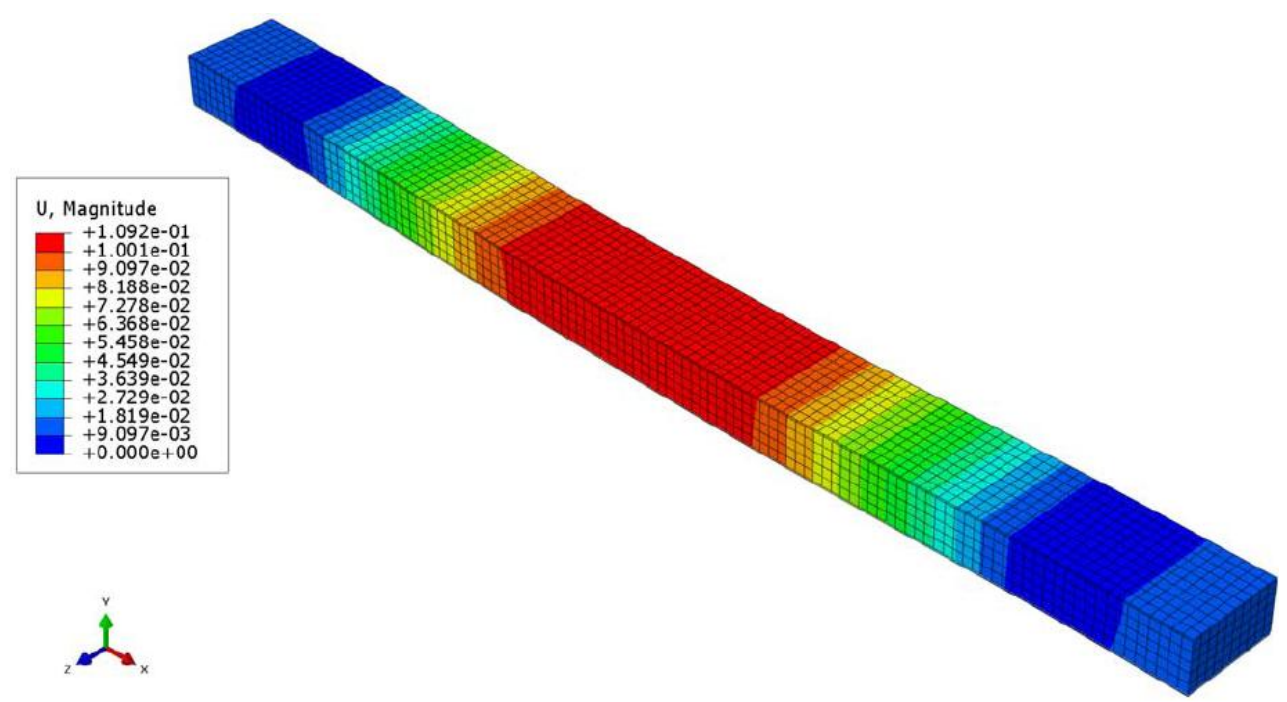

Figure 5.9. Displacement contour plot of control beam with deformation factor $=1$. 
The stress and displacements from the four point bending condition for the initially delaminated beam can be found in table 5.4 below.

Table 5.4. Results for four-point bending condition on delaminated beam.

\begin{tabular}{|l|l|}
\hline \multicolumn{2}{|l|}{ Mid Span Load $=2 \times 50$ lbs } \\
\hline Max Deflection & -0.1283 in \\
\hline Max Deflection -Experimental & -0.1095 in \\
\hline Max Beam von Mises & $13.48 \mathrm{ksi}$ \\
\hline
\end{tabular}

The delaminated beam did not produce significantly different beam displacements compared to non-delaminated control beam. The resulting deflection differs by $14.6 \%$ from the experimental test results. Although not significant, the delamination does cause a greater deflection in the beam. This can be seen in a contour plot of the beam deflection in fig. 5.10 below.

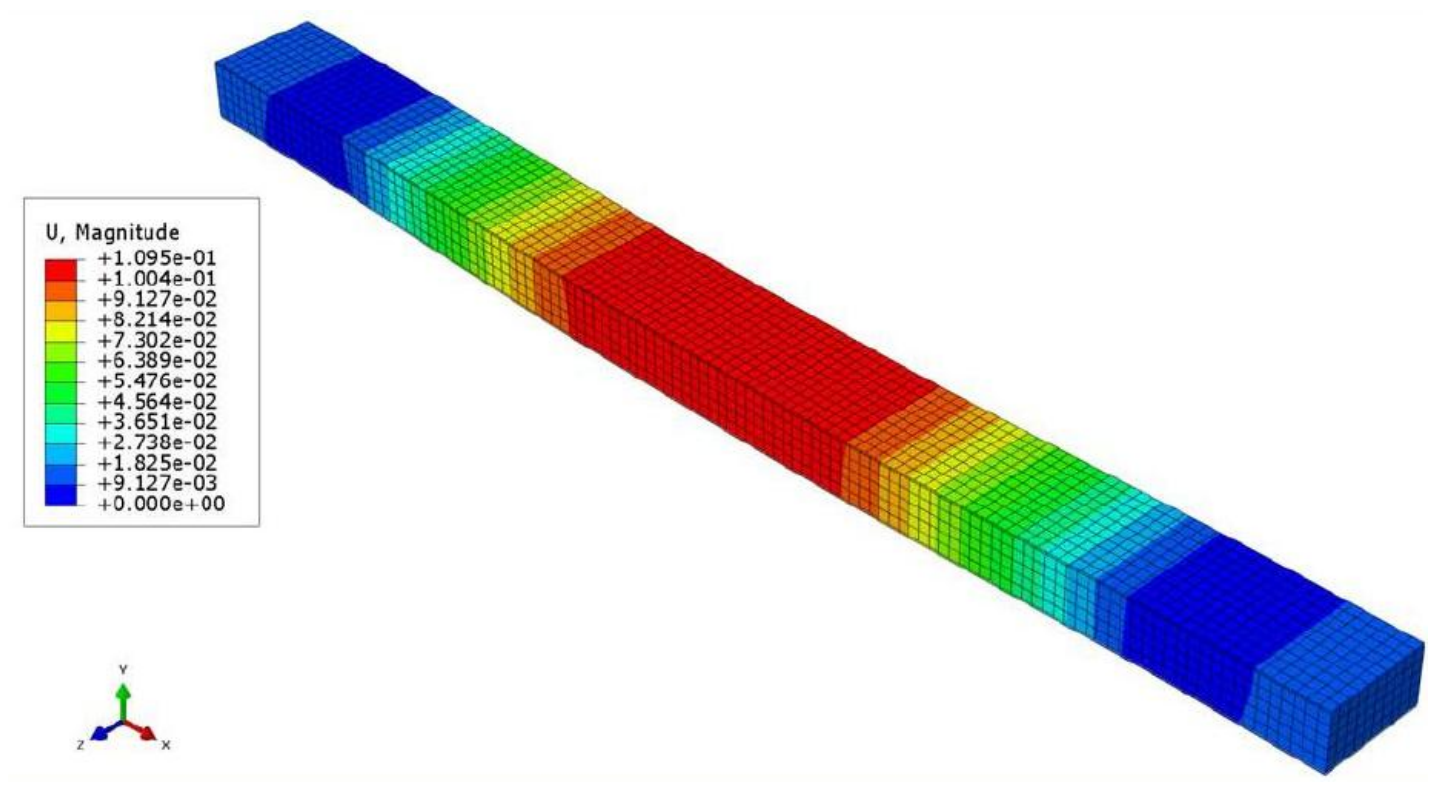

Figure 5.10. Displacement contour plot of delaminated beam case with deformation factor $=1$.

The stress and displacements of the non-delaminated beam with the addition of transverse DADs under four-point bending can be found in table 5.5 below. 
Table 5.5. Results for four-point bending condition on non-delaminated beam with transverse DADs.

\begin{tabular}{|l|l|}
\hline \multicolumn{2}{|l|}{ Mid Span Load $=2 \times 50$ lbs } \\
\hline Max Deflection & -0.1166 in \\
\hline Max Deflection- Experimental & -0.1089 in \\
\hline Max Beam von Mises & $13.49 \mathrm{ksi}$ \\
\hline
\end{tabular}

The resulting deflection matches the hand calculations within $6.6 \%$, indicating an acceptable model. This is slight increase in the maximum deflection of the beam compared to the control case, which follows what was seen in experiment with the addition of the transverse DADs. The beam deflection can be seen in fig. 5.11 below.

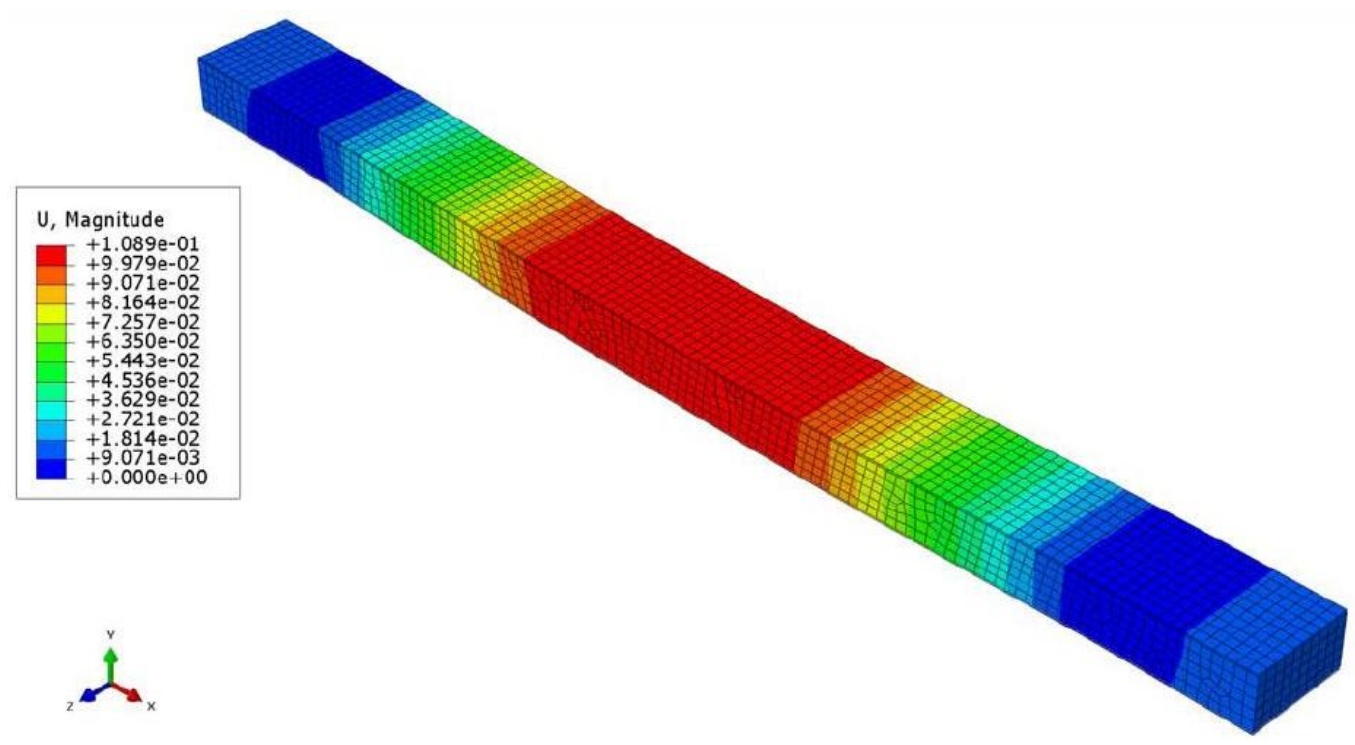

Figure 5.11. Displacement contour plot of transverse DAD key case with deformation factor $=1$. 
The deflection and stress results for the delaminated beam with transverse DADs can be seen below.

Table 5.6. Results for four-point bending condition on delaminated beam with transverse DADs.

\begin{tabular}{|l|l|}
\hline \multicolumn{2}{|l|}{ Mid Span Load $=2 \times 50$ lbs } \\
\hline Max Deflection & -0.1216 in \\
\hline Max Deflection- Experimental & -0.1089 in \\
\hline Max Beam von Mises & $13.49 \mathrm{ksi}$ \\
\hline
\end{tabular}

The resulting deflection matches the hand calculations within $10.6 \%$, indicating an acceptable model. This is a decrease in the maximum deflection of the beam compared to the delaminated beam case, which is expected with the addition of the transverse DADs in a flawed beam. The beam deflection can be seen in fig. 5.12 .

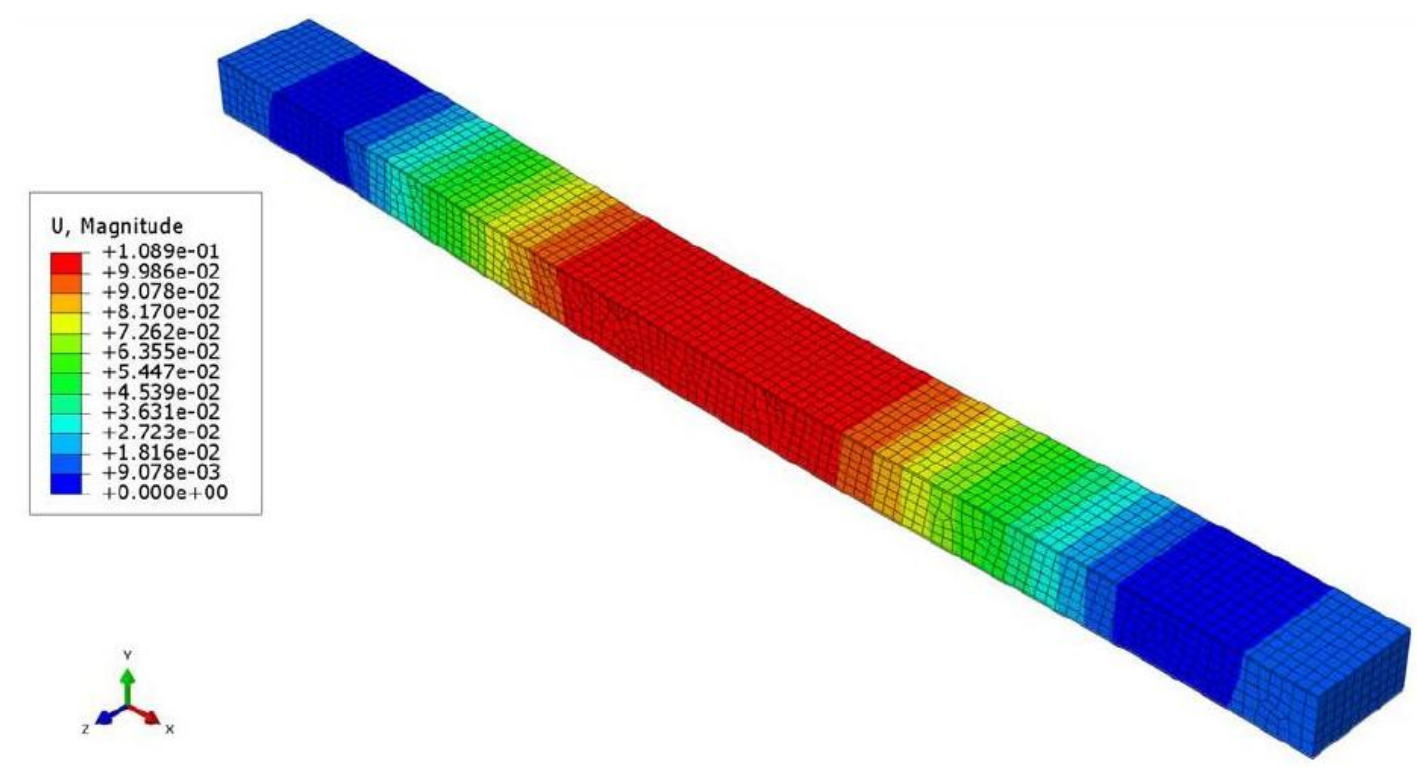

Figure 5.12. Displacement contour plot of delaminated beam with transverse dad keys with deformation factor = 1. 
The stress and displacements of the non-delaminated beam with the addition of a longitudinal DAD under four-point bending can be found in table 5.7 below.

Table 5.7. Results for four-point bending condition on non-delaminated beam with Transverse DADs.

\begin{tabular}{|l|l|}
\hline \multicolumn{2}{|l|}{ Mid Span Load $=2 \times 50$ lbs } \\
\hline Max Deflection & -0.0948 in \\
\hline Max Deflection- Experimental & -0.1014 in \\
\hline Max Beam von Mises & $17.46 \mathrm{ksi}$ \\
\hline
\end{tabular}

The resulting deflection matches the hand calculations within $6.9 \%$, indicating an acceptable model. This is a decrease in the maximum deflection of the beam compared to the control case, which is expected with the addition of the longitudinal DAD. The beam deflection can be seen in fig. 5.13 below.

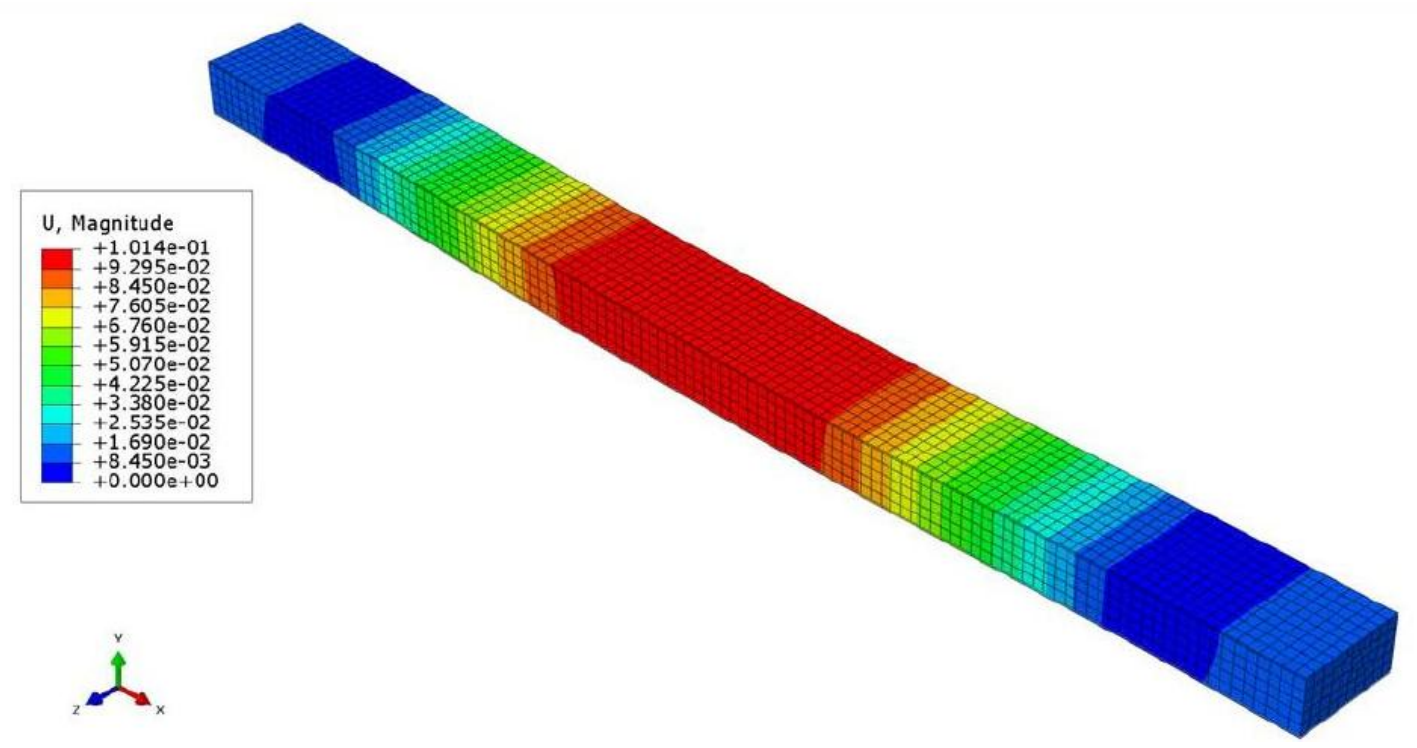

Figure 5.13. Displacement contour plot of longitudinal DAD key case with deformation factor $=1$. 
The deflection and stress results for the final configuration, delaminated beam with a longitudinal $\mathrm{DAD}$, can be seen below.

Table 5.8. Results for four-point bending condition on delaminated beam with Longitudinal DAD.

\begin{tabular}{|l|l|}
\hline \multicolumn{2}{|l|}{ Mid Span Load $=2 \times 50$ lbs } \\
\hline Max Deflection & -0.1169 in \\
\hline Max Deflection- Experimental & -0.1018 in \\
\hline Max Beam von Mises & $17.46 \mathrm{ksi}$ \\
\hline
\end{tabular}

The resulting deflection matches the hand calculations within $12.9 \%$, indicating an acceptable model. This is also a decrease in the maximum deflection of the beam compared to the delaminated control case, which is expected with the addition of the DAD in a flawed beam. The beam deflection can be seen in fig. 5.14 .

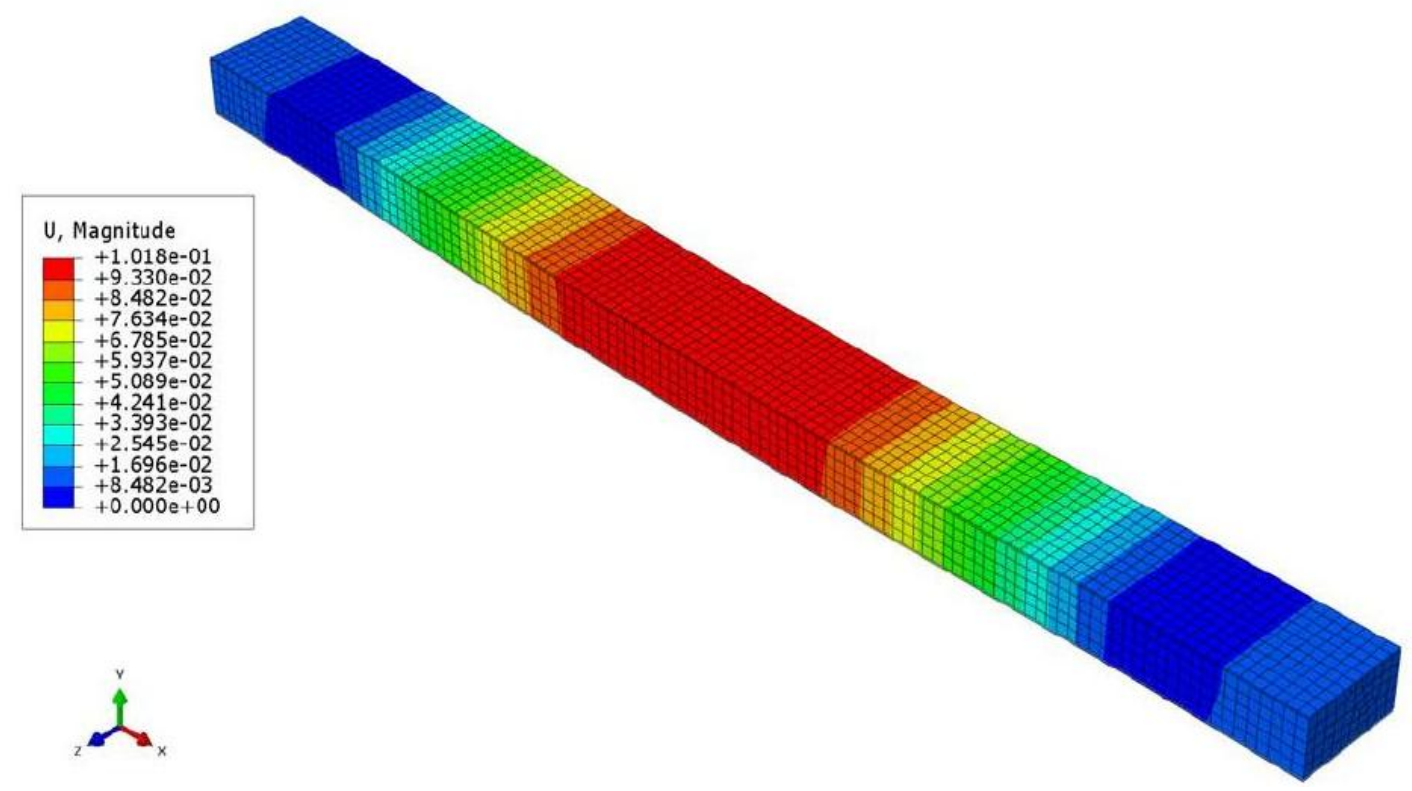

Figure 5.14. Displacement contour plot of delaminated beam with longitudinal DAD key with deformation factor = 1. 
Table 5.9. Comparison of all Numerical and Experimental Deflections.

\begin{tabular}{|c|c|c|c|c|c|c|}
\hline & \multicolumn{2}{|c|}{ Control } & \multicolumn{2}{|c|}{$\begin{array}{c}\text { Transverse } \\
\text { DAD }\end{array}$} & \multicolumn{2}{|c|}{$\begin{array}{c}\text { Longitudinal } \\
\text { DAD }\end{array}$} \\
\hline & $\begin{array}{c}\text { No } \\
\text { Delam }\end{array}$ & Delam & $\begin{array}{c}\text { No } \\
\text { Delam }\end{array}$ & Delam & $\begin{array}{c}\text { No } \\
\text { Delam }\end{array}$ & Delam \\
\hline Experimental (in) & -0.1001 & -0.1283 & -0.1166 & -0.1216 & -0.0948 & -0.1169 \\
\hline Numerical (in) & -0.1014 & -0.1095 & -0.1089 & -0.1089 & -0.1014 & -0.1018 \\
\hline Percent Difference & 1.3 & 14.6 & 6.6 & 10.6 & 6.9 & 12.9 \\
\hline
\end{tabular}

The experimental deflection values for each beam configuration under four-point bending were fairly similar to those found in the FE model. The fact that the values found numerically differ $1-14 \%$ from the values of deflection found experimentally adds to the confidence that the simulation was successful. In addition, for standard displacement based elements, the response is less stiff than the actual values. The error is likely due to the fact that the mechanical characteristics of the foam obtained through experiment have a significantly lower elastic modulus than the given value from the manufacturer. Because the foam was stacked (which is not part of the ASTM standard) when testing for the mechanical characteristics of the foam, the results of the test were not realistic because they yielded a much lower elastic modulus than the manufacturer provided. Therefore, when the elastic modulus from experiment is used, the model becomes less stiff and the deflections found resulted in greater values. For research purposes, the elastic modulus value found from manufacturer of the foam was used in the model and the same amount of error resulted but in this case the beams were too stiff and all the deflections were about 1-14 percent larger, as seen in table 5.9. A recommendation would be to obtain a single sample of the foam with the correct depth values called by the ASTM test to avoid stacking and obtain the correct mechanical properties of the samples (which would probably be in between the values tested and the values supplied by the manufacturer) and use that for the simulation. Since the FE model yielded acceptable deflection results, maximum stress in the beams were plotted for the beam Von Mises and each beam configuration can be found in Appendix B. 


\section{Non Delaminated Configurations}

When subjected to the total static downward force of 100 -lbs, the non-delaminated control beam deflected most at the mid-span of the beam. After the addition of the transverse DADs, the deflection increased by approximately $7.4 \%$. This is not a significant increase, which is expected since there was no initial delamination in the beam. With the addition of the transverse DADs, the maximum stress that occurs at the supports increases. After the addition of the longitudinal $\mathrm{DAD}$, the deflection did not increase. This followed what occurred in the experiment closely because the addition of the DADs to an unflawed test specimen did not typically enhance the test specimen. With the addition of the longitudinal DAD, the maximum stress that occurs at the supports increases as well.

\section{Delaminated Configurations}

In the case of the delaminated control beam, the addition of the DADs provided a decrease in the beams maximum deflection. The FE results showed that there was a decrease in beam deflection for the transverse DAD keys and longitudinal DAD key of approximately $0.6 \%$ and $7 \%$, respectively. These results followed the experimental results found in the four point bending test. However, in the experiment the addition of the transverse DAD keys proved to be more effective in improving the beams ability to withstand more load.

In the experimental testing it was found the there was a significant increase in ultimate strength of the beam with the addition of the DADs. Since the ASTM standards used to find the ultimate strengths and other mechanical data use equations which are functions of the beam deflection, the deflections found numerically must follow closely to those in experiment to ensure the testing is valid. In this research, the experimental deflection values found are validated with the deflections found through FE.

The six different configured composite sandwich beam models illustrate how simple fiberglass inserts can affect stress and displacement values. All models have a net $100 \mathrm{lbs}$ downward force applied to the same location on the beam, but each is configured differently. The 
addition of the DADs in a delaminated beam, affect the displacement quite significantly, whereas the DADs do not affect the deflections very much in a non-delaminated beam. The addition of the DADs affects the maximum stress seen in the beam in both cases. 


\section{Chapter 6 Conclusion}

The objective of this study was to determine the effectiveness of damage arrestment devices placed in composite sandwich structures subjected to 4-point bend fatigue loading, after a low velocity impact. Further, static 4-point bend testing was also performed to determine whether DADs increased the ultimate strength of the composite sandwich structures with an initial face-core delamination. A 4-point bend fatigue test was implemented in order to determine if the DAD keys increased fatigue life of the impacted composite sandwich beams and those with an initial face-core delamination.

\section{The static 4-point bend test showed:}

- Adding a layer of sheet resin between the foam core and face sheet, as seen in [21], greatly improved the effectiveness of DAD keys on the structural integrity of both an initially delaminated and non-delaminated composite sandwich structure.

- The ultimate strength of the composite sandwich structures decreased greatly when introduced to a low-velocity impact.

- $D A D$ keys significantly improved the ultimate strength of an initially delaminated composite sandwich structure under 4-point bend.

- No failure was visible in the face sheet due to bending, indicating that the flexural strength of the structure was mostly dependent on the flexural strength of the foam core and the shear strength of the face-core bonding interaction.

Based on these results, it is recommended that the designer consider that the addition of DADs significantly increases the strength under bending of composite sandwich structures that are flawed due to delamination. 


\section{The 4-point bend fatigue test showed:}

- The fatigue life of sandwich composite structures with and without delamination is significantly extended through the introduction of transversely placed DADs.

- DAD keys greatly increased the fatigue life of previously impacted composite sandwich structures and composite sandwich structures with an initial presence of delamination.

- Initially delaminated structures were greatly affected by the introduction of both longitudinally and transversely placed DAD keys, with a considerable increase in fatigue life.

- The flexural strength of previously impacted composite sandwich beams is directly driven by the strength of the carbon fiber face sheet.

Based on these results, it is recommended that the designer consider that the addition of DADs significantly prolongs the fatigue life of a flawed composite sandwich structure, including an impacted composite sandwich structure or a delaminated composite sandwich structure.

In summary, these results lead to the conclusion that the presence of DAD keys will increase the performance of flawed composite sandwich structures under bending. 


\section{References}

${ }^{[1]}$ Jones, Robert M. Mechanics of Composite Materials. 2nd ed. Philadelphia, PA: Taylor \& Francis, 1999. Print.

${ }^{[2]}$ Vasiliev, Valery V., and Evgeny V. Morozov. Mechanics and Analysis of Composite Materials. New York: Elsevier Science, 2001. Print.

[3] "Ultrasonic Wave Interaction with Heterogeneous Material." Modelling and Advanced Software. University of Strathclyde Engineering. Web. 20 September 2012. $<$ http://www.strath.ac.uk/eee/research/cue/research/modellingandadvancedsoftware/ultra sonicwaveinteractionwithheterogeneousmedia/>.

[4] "Bamboo Strip as Orthotropic Unidirectional Fibrous Composite." Bamboo Structural Material. Luit Nirman. Web. 20 September 2012. <http://www.assambambooworld.com/bamboostructural-material.htm>.

${ }^{[5]}$ Niu, Michael Chun-Yung. Composite Airframe Structures: Practical Design Information and Data. North Point: Hong Kong Conmilit, 1992. Print.

[6] "Sandwich Constructions." About Composites. Advanced Lightweight Engineering Web. 01 October 2012. <http://www.ale.nl/?portfolio=sandwich-constructions >.

${ }^{[7]}$ Callister, W.D., "Materials Science and Engineering: An Introduction $7^{\text {th }}$ Ed. Chapter 16" Print.

[8] Weekend Wings \#37: The F-111 Aardvark, Part 1. Web. 01 October 2012. <http://bayourenaissanceman.blogspot.com/2010/11/weekend-wings-37-f-111-aardvarkpart-1.html>.

[9] Broutman, Lawrence. "Impact Strength and Toughness of Fiber Composite Materials." Air Force Office of Scientific Research. Print.

${ }^{[10]}$ Reifsnider, K.L.. "Fatigue of Composite Materials" LS-DYNA Anwenderforum (1991). Volume 4 of Composite Materials series. Print. 
[11] "Fatigue Behavior of Composite Materials." Internal Journal of Fracture. SpringerLink Web. 01 October 2012. <http://link.springer.com/article/10.1007\%2FBF02265218>.

${ }^{[12]}$ Soni, Gibson, and Ayorinde. "Characterization of Fatigue Damage in Composite Sandwich Hull Materials at Low Temperatures." Wayne State University. Print.

${ }^{[13]}$ Roosen, Dirk. "Fatigue Behaviour of Sandwich Foam Core Materials- Comparison of Different Core Materials." Print.

${ }^{[14]}$ Harte, Fleck, and Ashby. "The fatigue strength of sandwich beams with an aluminum alloy foam core." International Journal of Fatigue. Print.

${ }^{[16]}$ Butler. "Post-Buckling and Fatigue Limit of Artificially Delaminated Composites." AIAA. Print.

[17] Balatbat, Richard. An Investigation of Damage Arrestment Devices Application with Fastner/Hole Interaction. Thesis. California Polytechnic State University, San Luis Obispo, September 2010. Print.

${ }^{[18]}$ Surano, Dominic. The Effectiveness of Damage Arrestment Devices in Delaying FastenerHole Interaction Failures in Carbon Fiber Polyurethane Foam Composite Sandwich Panels Subjected to Static and Dynamic Loading Under Increased Temperatures. Thesis. California Polytechnic State University, San Luis Obispo, September 2010. Print.

[19] Tran, Tony D. An Investigation of Initially Delaminated Composite Sandwich with Delamination Arrest Mechanism Under Buckling Loading. Thesis. California Polytechnic State University, San Luis Obispo, September 2010. Print.

${ }^{[20]}$ Eswonia, Eugene. Effects of Delamination on Composite Structures under Static and Fatigue Loading. Thesis. Califorina Polytechnic State University, San Luis Obispo, December 2009. Print.

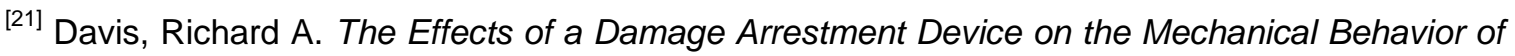
Composite sandwich Beams under Four-Point Bending. Thesis. California Polytechnic State University, San Luis Obispo, May 2011. Print.

[22] Rider, Kodi. The Effect of Low Velocity Impact on the Flexural Strength and Dynamic Response of Composite Sandwiches with Damage Arrestment Devices. Thesis. California Polytechnic State University, San Luis Obispo, August 2012. Print. 
[23] "Standard Test Method for Tensile Properties of Polymer Matrix Composite Materials." ASTM International (2008): 1-13. Print.

[24] "Standard Test Method for Poisson's Ratio at Room Temperature." ASTM International (2010): 1-3. Print.

[25] "Standard Test Method for Compressive Properties of Rigid Cellular Plastics." ASTM International (2010): 1-5. Print.

[26] "Standard Test Method for Compressive Properties of Polymer Matrix Composite Materials with Unsupported Gage Section by Shear Loading." ASTM International (2008): 1-16. Print.

[27] "Standard Test Method for Flexural Properties of Unreinforced and Reinforced Plastics and Electrical Insulating Materials by Four-Point Bending." ASTM International (2008): 1-8. Print.

[28] " Standard Test Method for Measuring the Damage Resistance of a Fiber-Reinforced Polymer Matrix Composite to a Drop-Weight Impact Event." ASTM International (2007): 1-16. Print.

${ }^{[29]}$ American Society of Testing and Materials, Applications of Composite Materials STP 524, $1^{\text {st }}$ ed., Library of Congress, Tallahassee FL., 1973, Chap. 1

[30] "ACG LTM 40 Series Component Prepregs." Advanced Composites Group PDS 1042 (2007): 1-14. Print. 


\section{Appendix}

See Attached. 


\section{Appendix A}

Safety Precautions:

\section{Autoclave Operations}

If Alarm sirens

- Reset alarm on software, otherwise the alarm will stop within 15 seconds

Do NOT press Emergency stop button

- If done, all control over the Autoclave will be lost and the connection to the computer will be lost

On Power Box, "Heater High Limit" and "Fan High Limit" reset buttons will reset the max temperature and pressure limits. Currently, the max temperature is set to $T_{\max }=600^{\circ} \mathrm{F}$

To clean the Autoclave: Use hand vacuum to eliminate loose particles

Instructions for Use:

1. Begin by turning on the autoclave by switching point 1 and 2 in fig. 1 to the ON position.

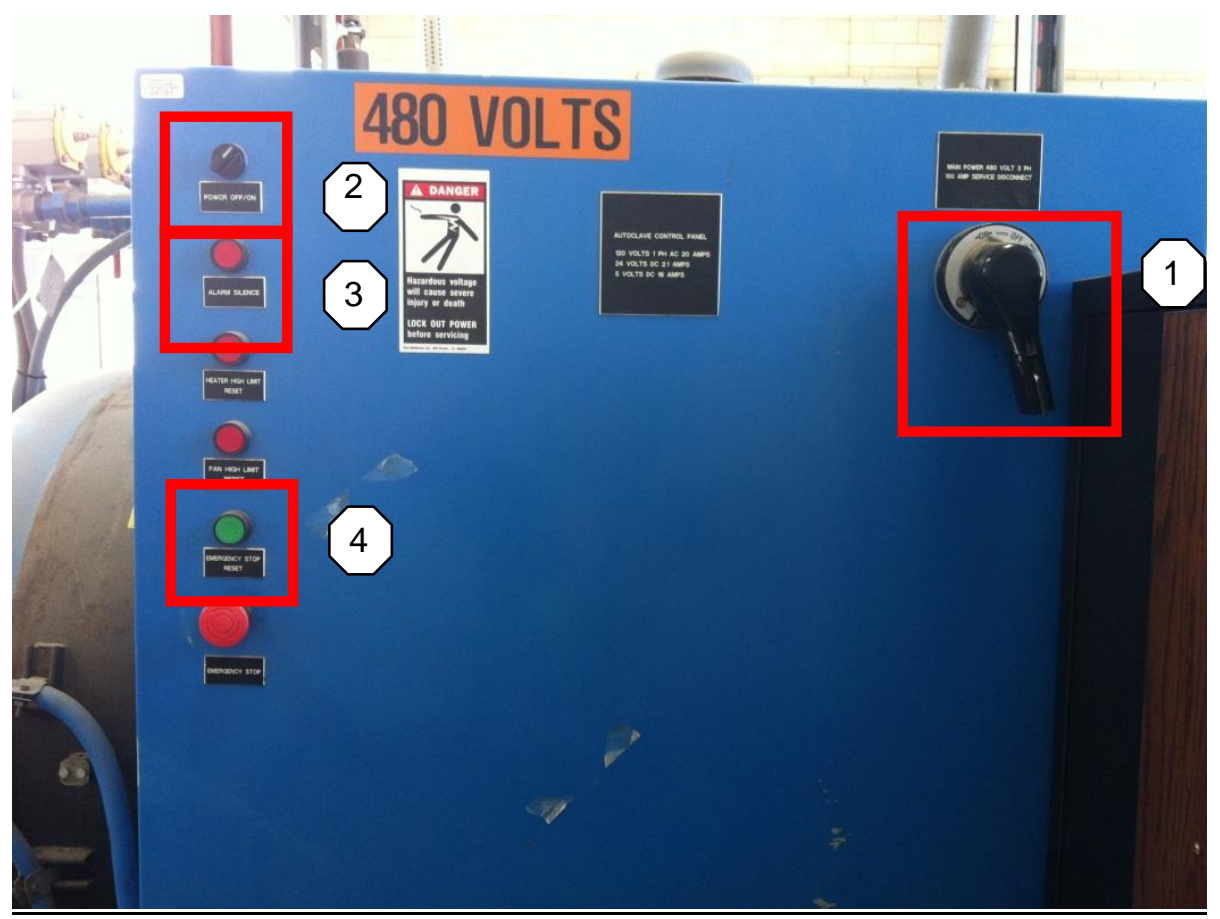

Figure 1. Autoclave Control Panel

2. Turn on computer and monitor located adjacent to the control panel.

3. American Autoclave Company screen will come up once the computer has turned on. Click the button "Click here to Continue" to login as shown in fig. 2. Enter the following login and password:

Operator ID:

oper123 
Password:

oper123

Upon entering, Press Send.

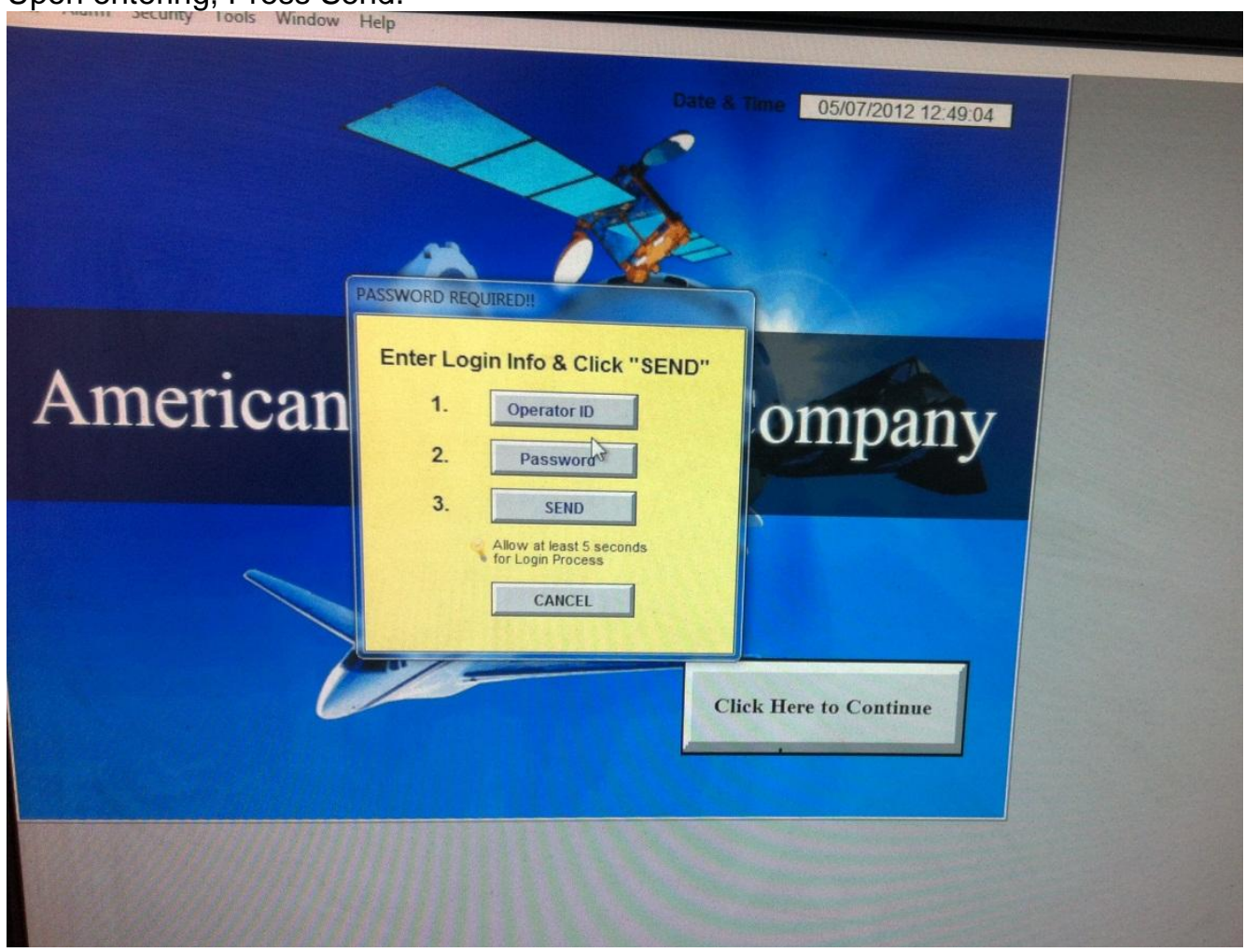

Figure 2. Start-up page

4. Once logged in, go to "Alarm Screen" and clear all errors. Press "Man in autoclave reset" button on alarm screen. Alarm will siren and press "Alarm Silence" as seen in fig. 1 at point 3 . Following, press "Alarm Reset" as shown in fig. 1 at point 4 . This will cause an automatic logout from the computer program.

5. Login in similarly to procedure in step 3.

6. Once logged in, there will be a grey screen. To bring program back up, go to File->Open Project->AutoClave1.UII->Open as shown in Fig. 3. This will allow for control of the autoclave through the computer.

7. We can now place the part in the autoclave and begin the external vacuum.

External vacuum:

Turn Valve 1 on(On position will be turning the valve handle towards operator), followed by turning on Vacuum 1(external vacuum as shown in Fig. 4)

- Note: Valve 1 and 2 can both be turned on, but each vacuum correlating must also be turned on, e.g. If Valve 2 is turned on, Vacuum 2 must also be turned on. There are two gauges that correlate to each valve to monitor the pressure. [Gage 1 is on the left, Gage 2 is on the right] 


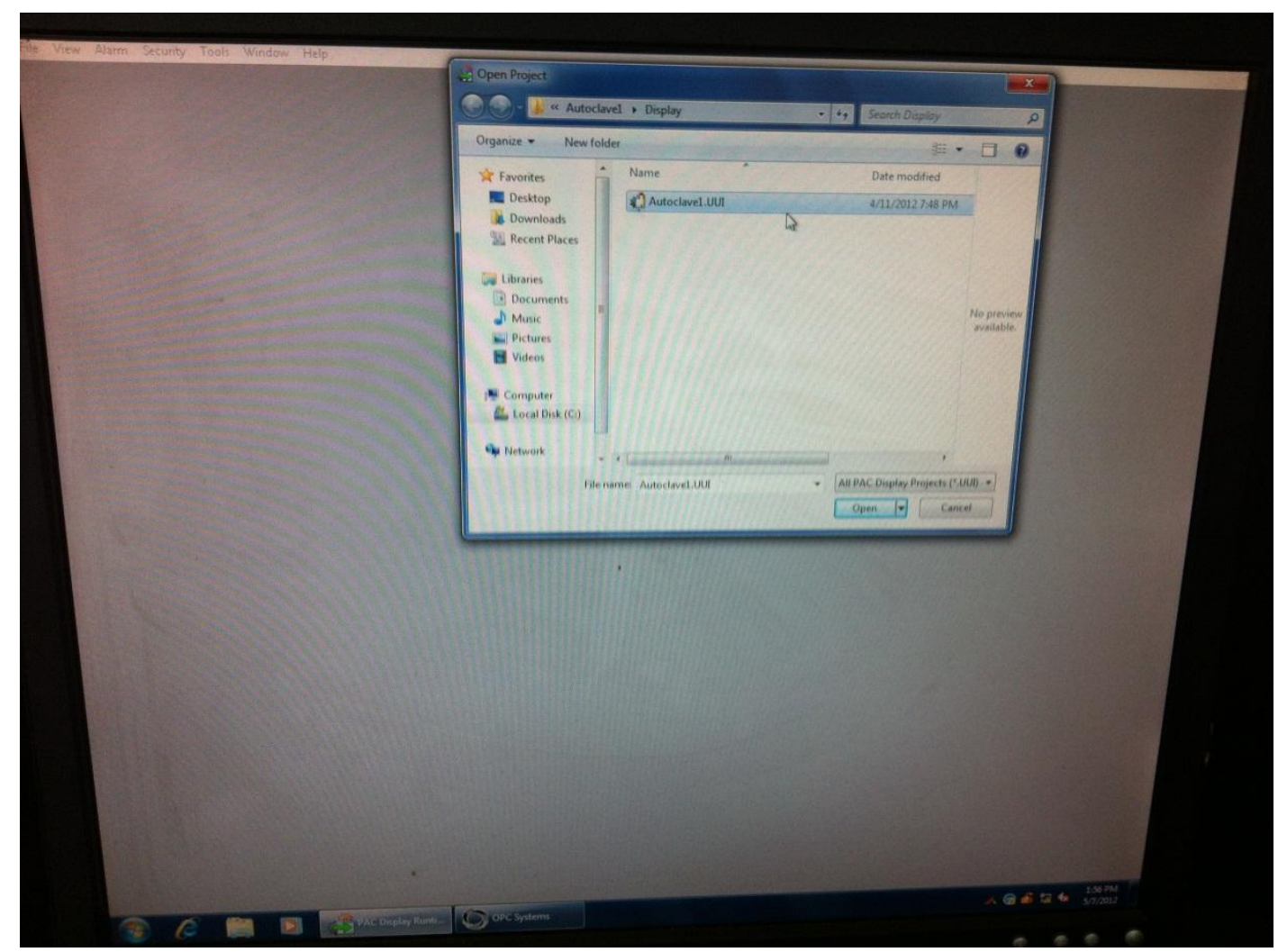

Figure 3. Screen shot of computer to open program manually.

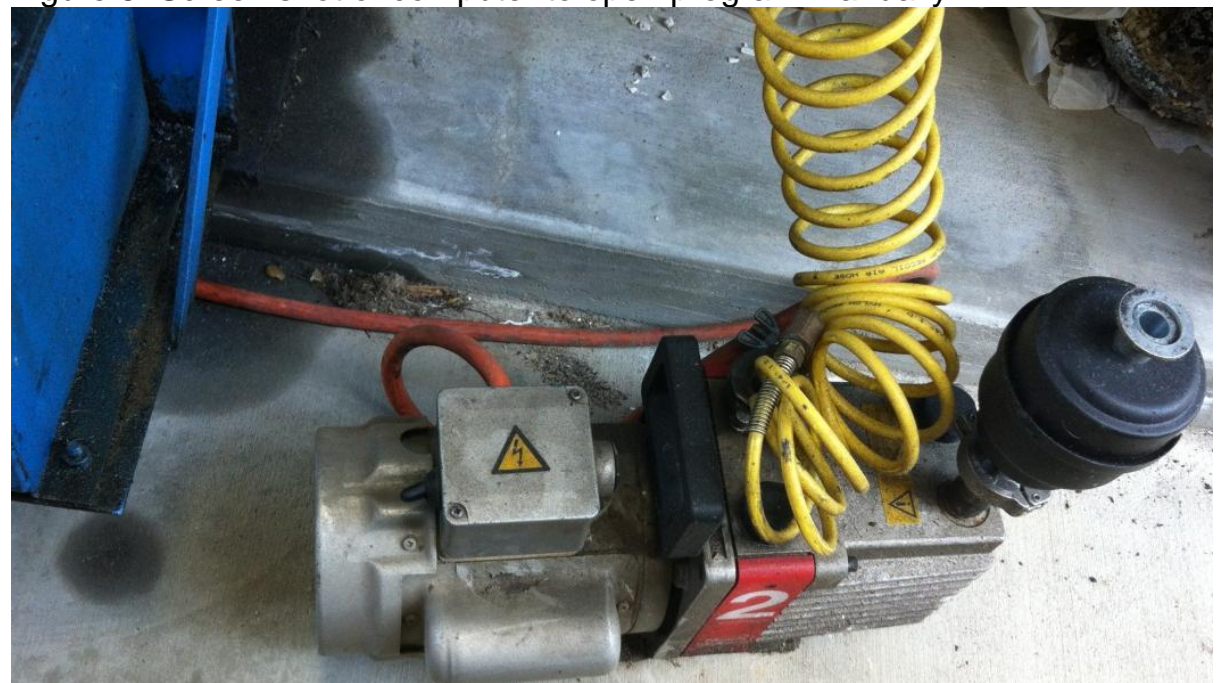

Figure 4. External vacuum pump

8. Turn on Hydraulic Pump switch as shown in fig. 5. 


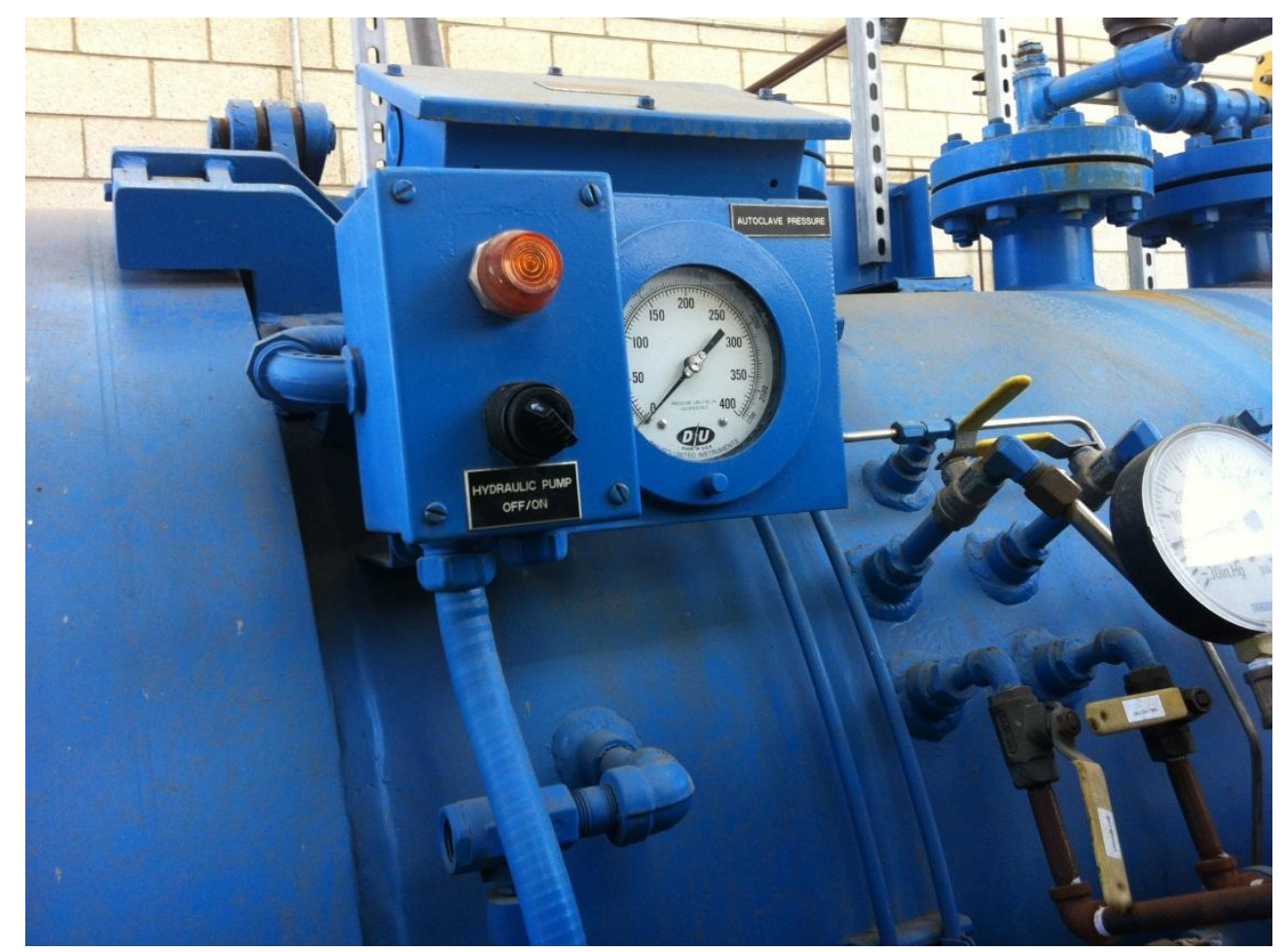

Figure 5. Hydraulic Pump Switch

9. Pull handle as shown in fig. 6 to open position.

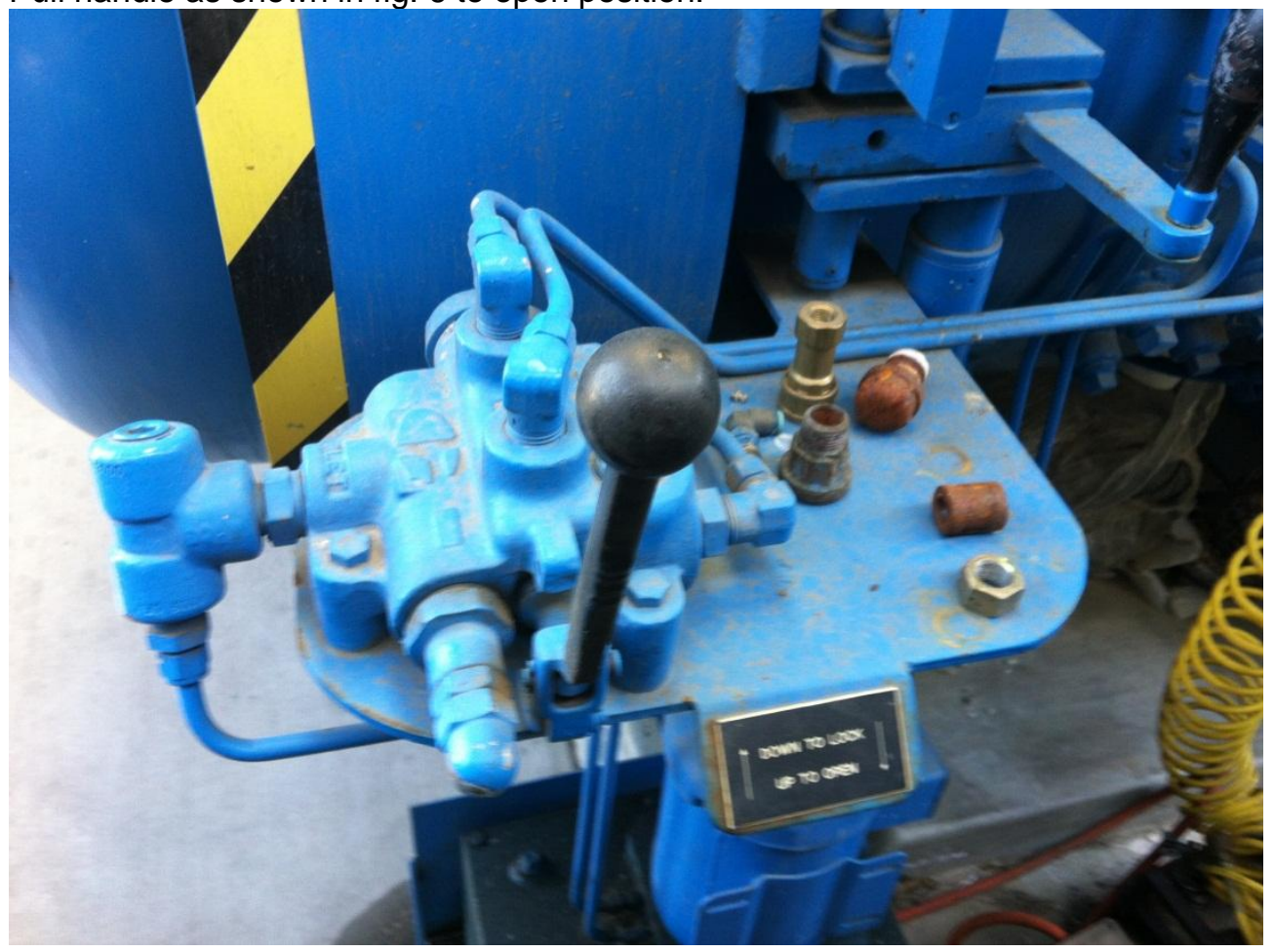

Figure 6. Black handle to open autoclave door.

10. Open door using black handle, shown in fig.6, until a "screeching" sounds is made which means it is free to open- This means Stop! (Instructions are located on a black plate) $\uparrow$ To Open $\downarrow$ To Lock

11. Pull Autoclave door open and place desired part in chamber as shown in Fig 7 and 8. Hook up each hose to part, one for the external vacuum and the other for the internal. 
Part should begin to vacuum and check for any leaks in the vacuum bag. Once this has been achieved the autoclave door can be closed.
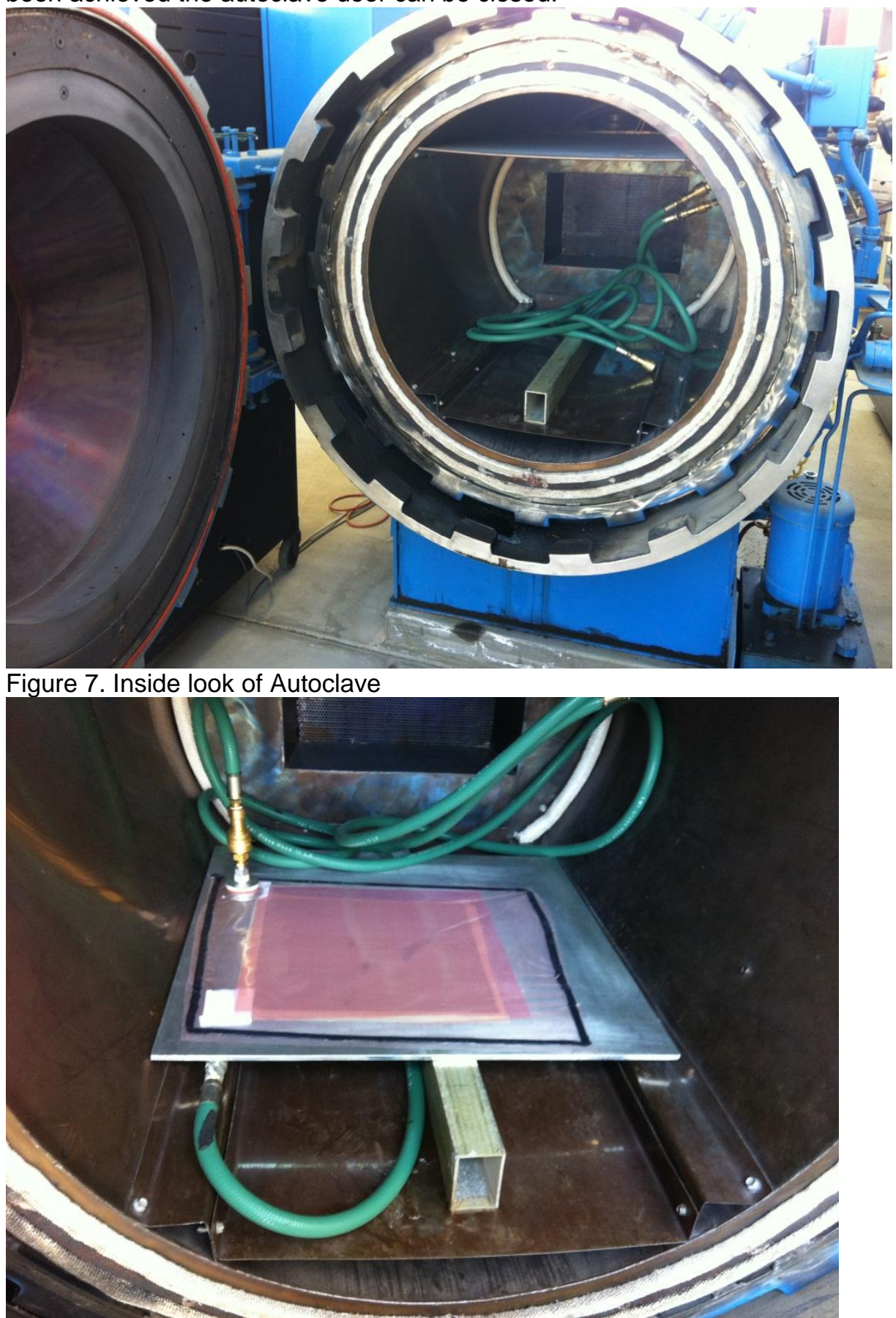

Figure 8. Part inside autoclave with hoses attached

12. Close door and to lock push down on black handle until a "screeching" sounds is made which means it has locked- This means Stop! (Instructions are located on a black plate) $\uparrow$ To Open $\downarrow$ To Lock

13. Use black handle mentioned in step 10 to lock the door by pulling the handle to the right into the lock position, where you can see a locking mechanism (Pin is in place).

14. Turn off hydraulic pump 
15. Return to computer screen. Upon entering program, select "Cure Control" as seen in fig. 9 and select "Press to start" shown in step 3 on the prompt screen. The cure cycle will now begin and the "Push to start" button should change to "Running" as shown in fig.10.
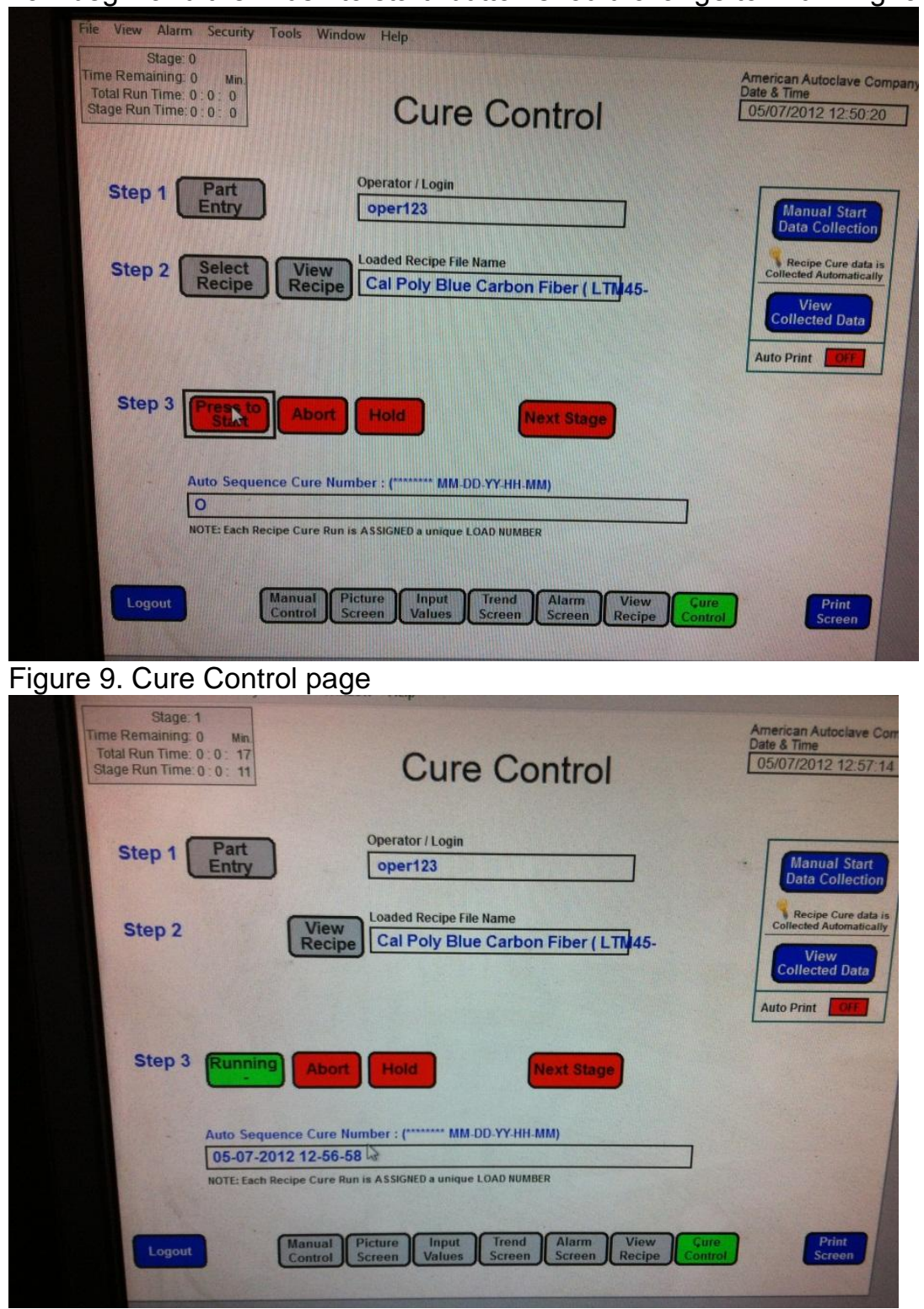

Figure 10. Cure Control page-running cure cycle

16. After the desired pressure has been reached (Apprx $10 \mathrm{~min}$ ) within the autoclave, turn off external pump (top valve) and turn on the bottom valve to release the gas.

\section{Upon Completion of Curing:}

Observe that the "Autoclave Pressure" is at 0 psi and pressure reading on the computer program also reads 0 psi.

Turn on hydraulic pump and pull lever on top towards door. Check to make sure there is no air leak by placing hand over valve (near door).

Clean inside of autoclave with a hand vacuum to pick up any loose particles. 


\section{Appendix B}

Von Mises Contour Plots:

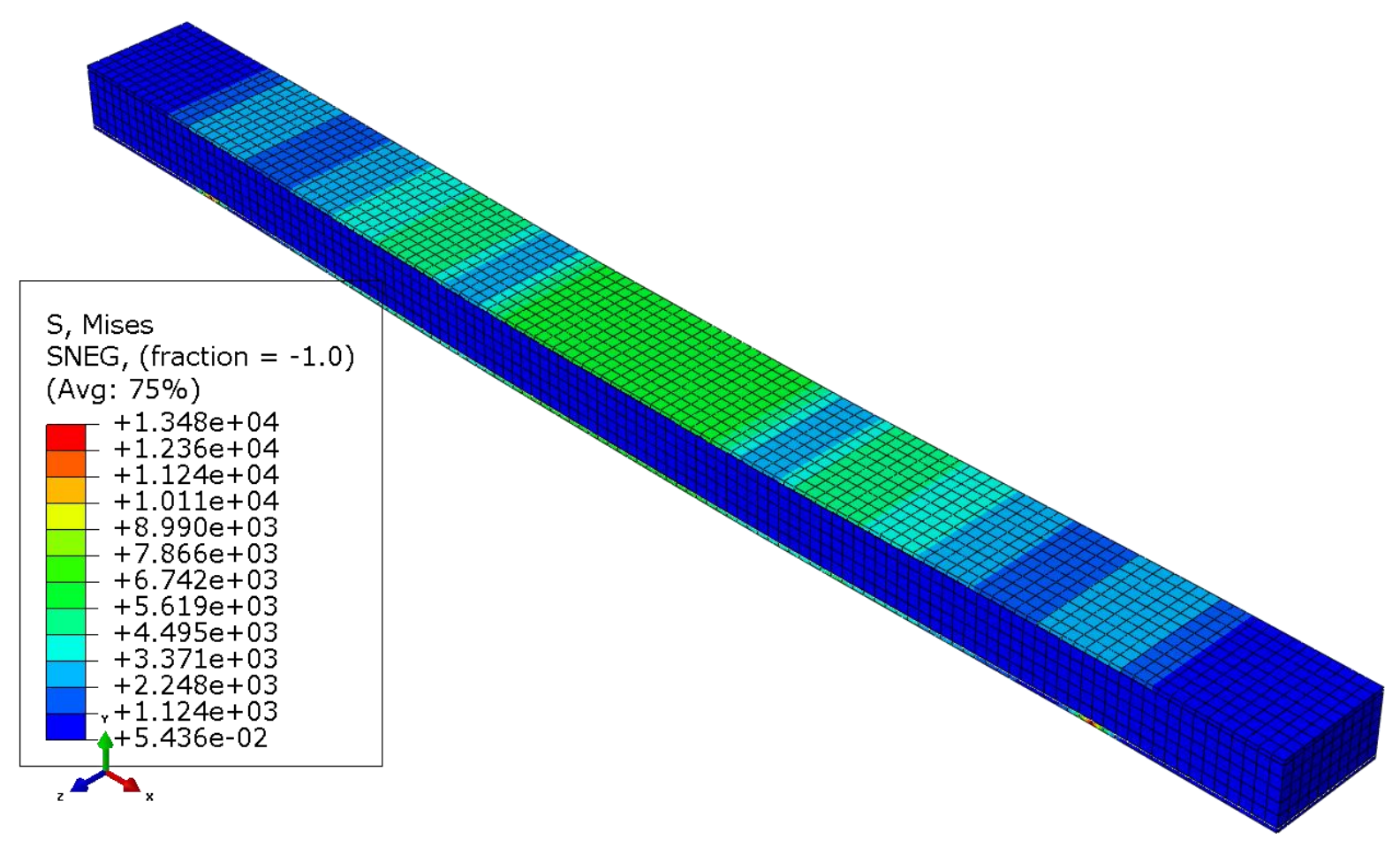

Figure 1. Von Mises non-delaminated control beam 


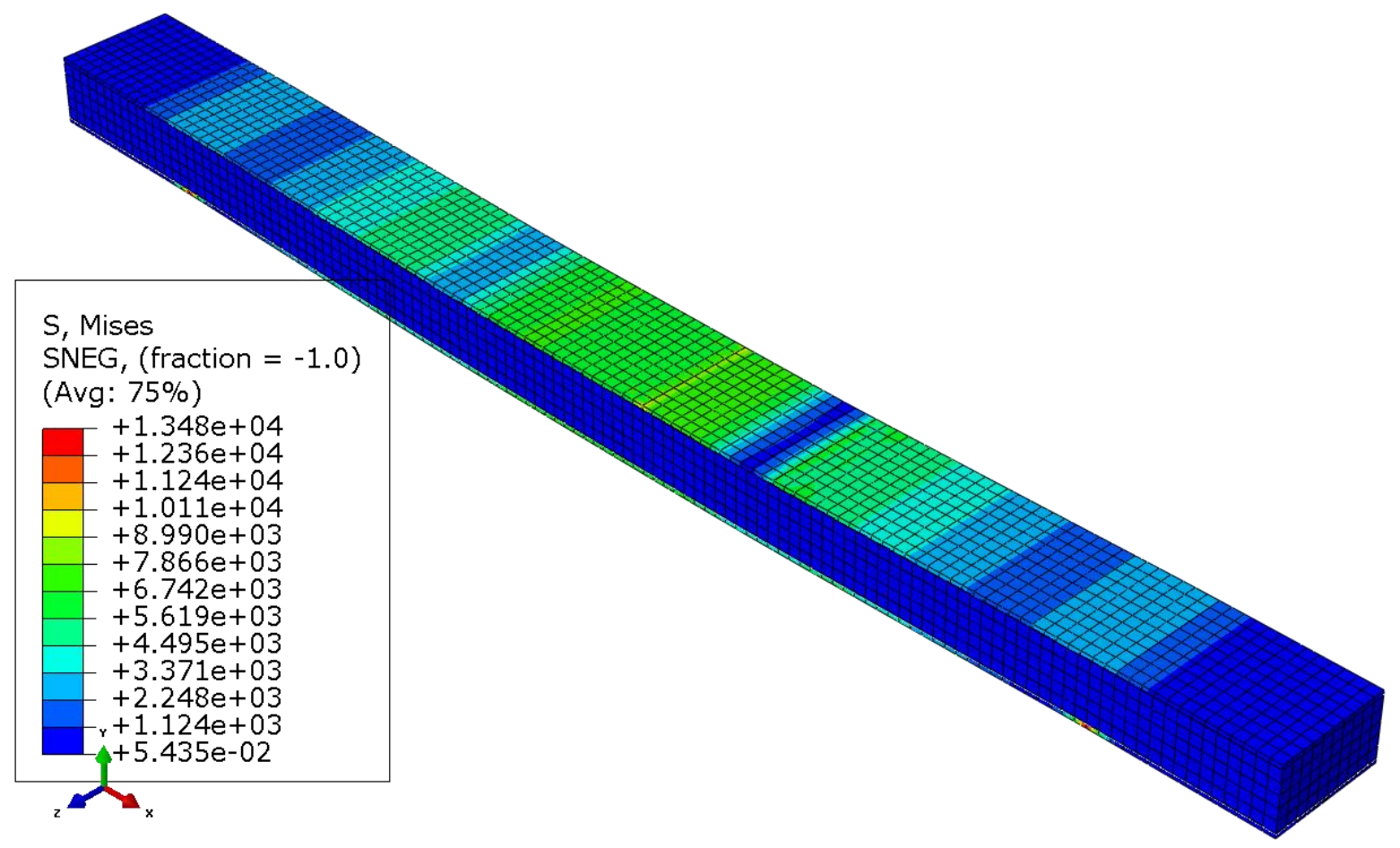

Figure 2. Von Mises delaminated control beam

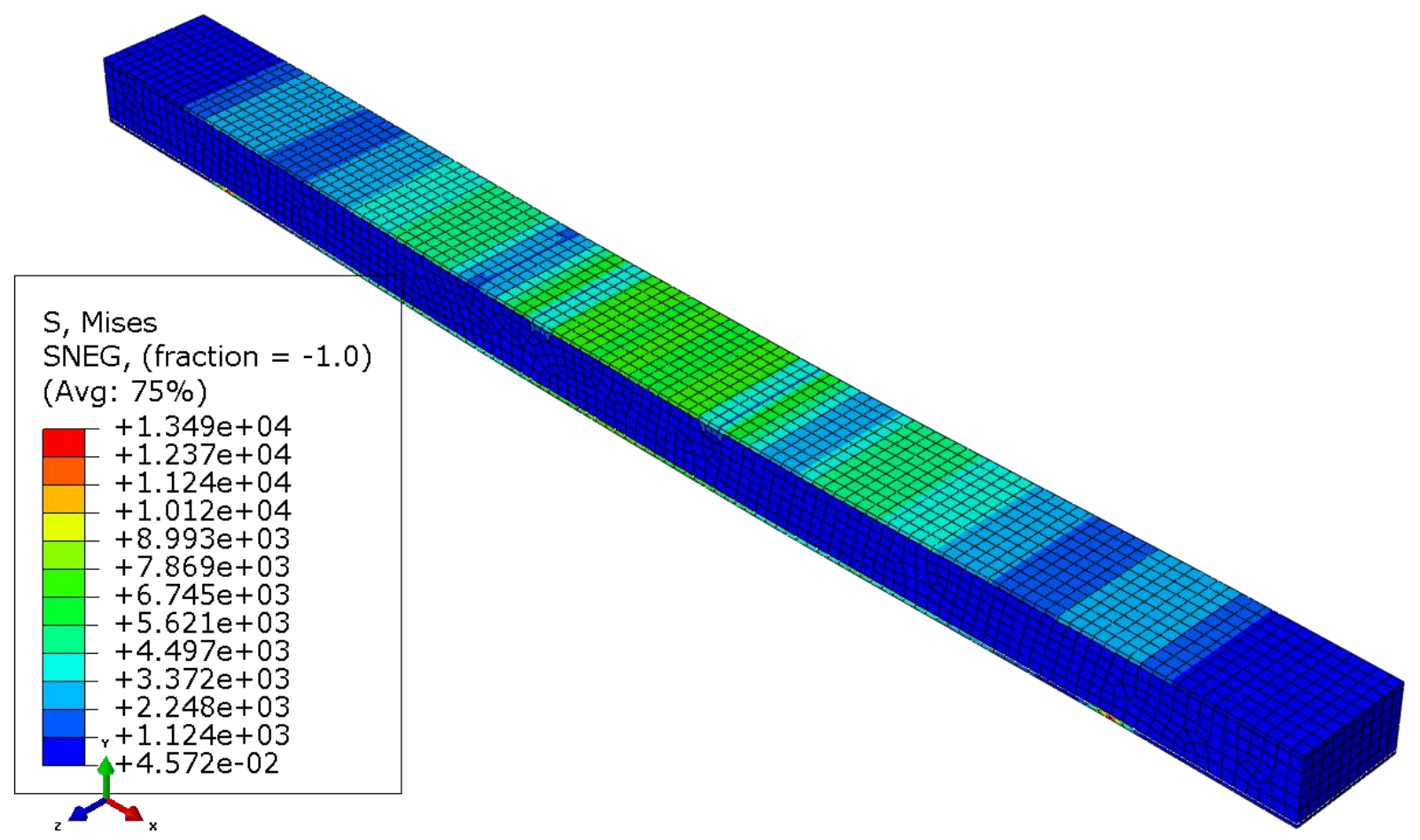

Figure 3. Von Mises non-delaminated beam with transverse DADs 


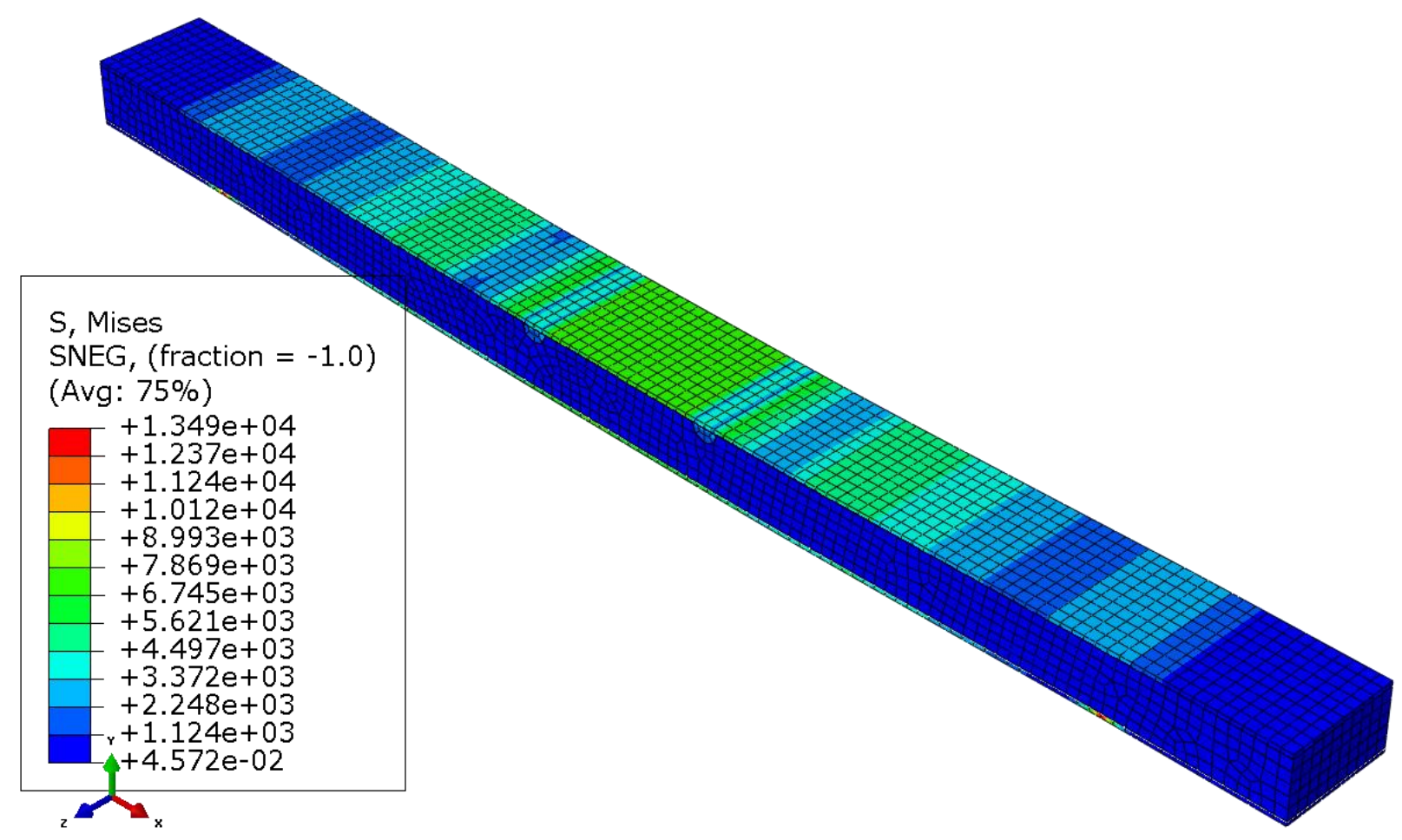

Figure 4. Von Mises delaminated beam with transverse DADs

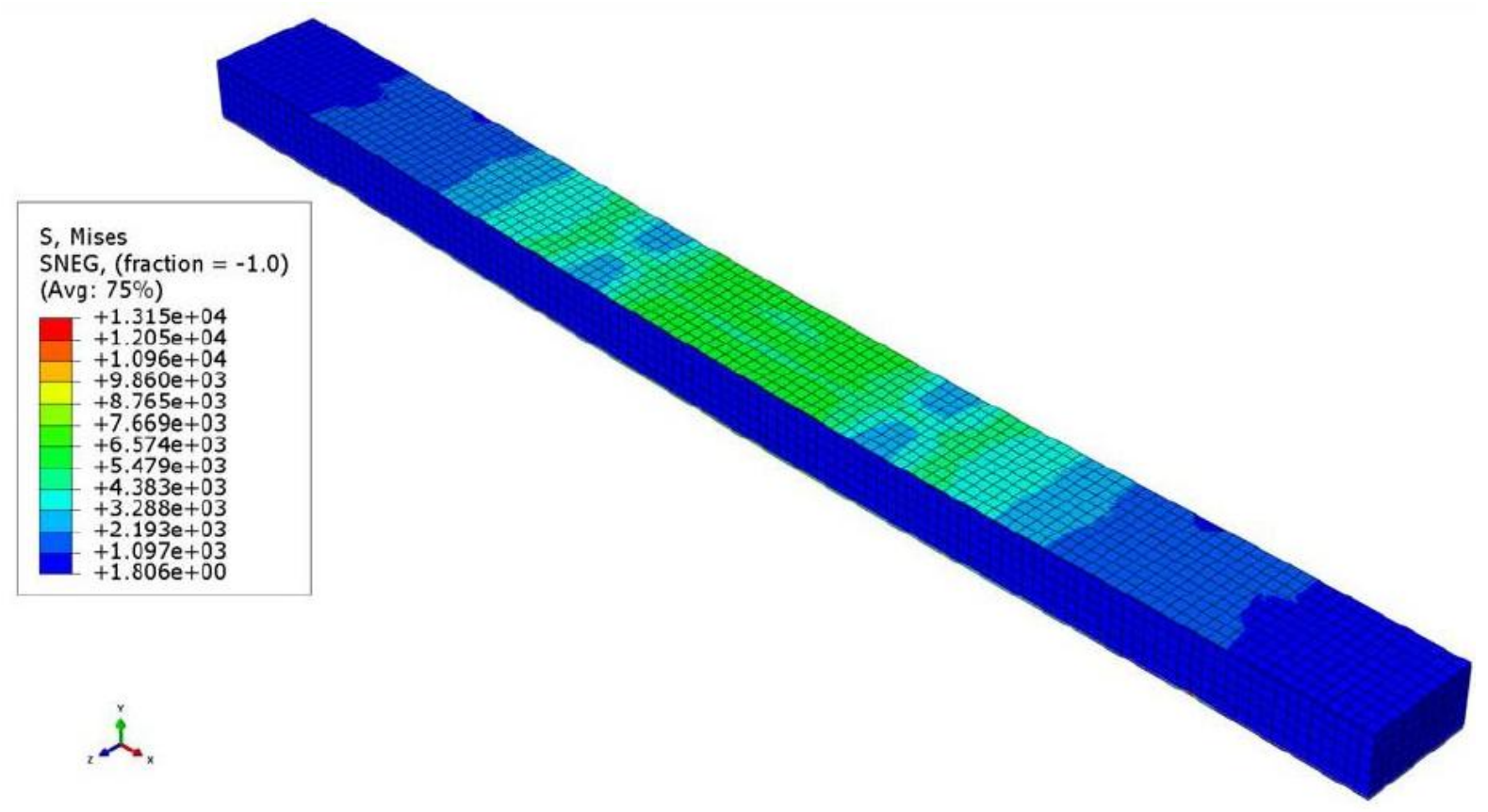

Figure 5. Von Mises non-delaminated beam with longitudinal DADs 


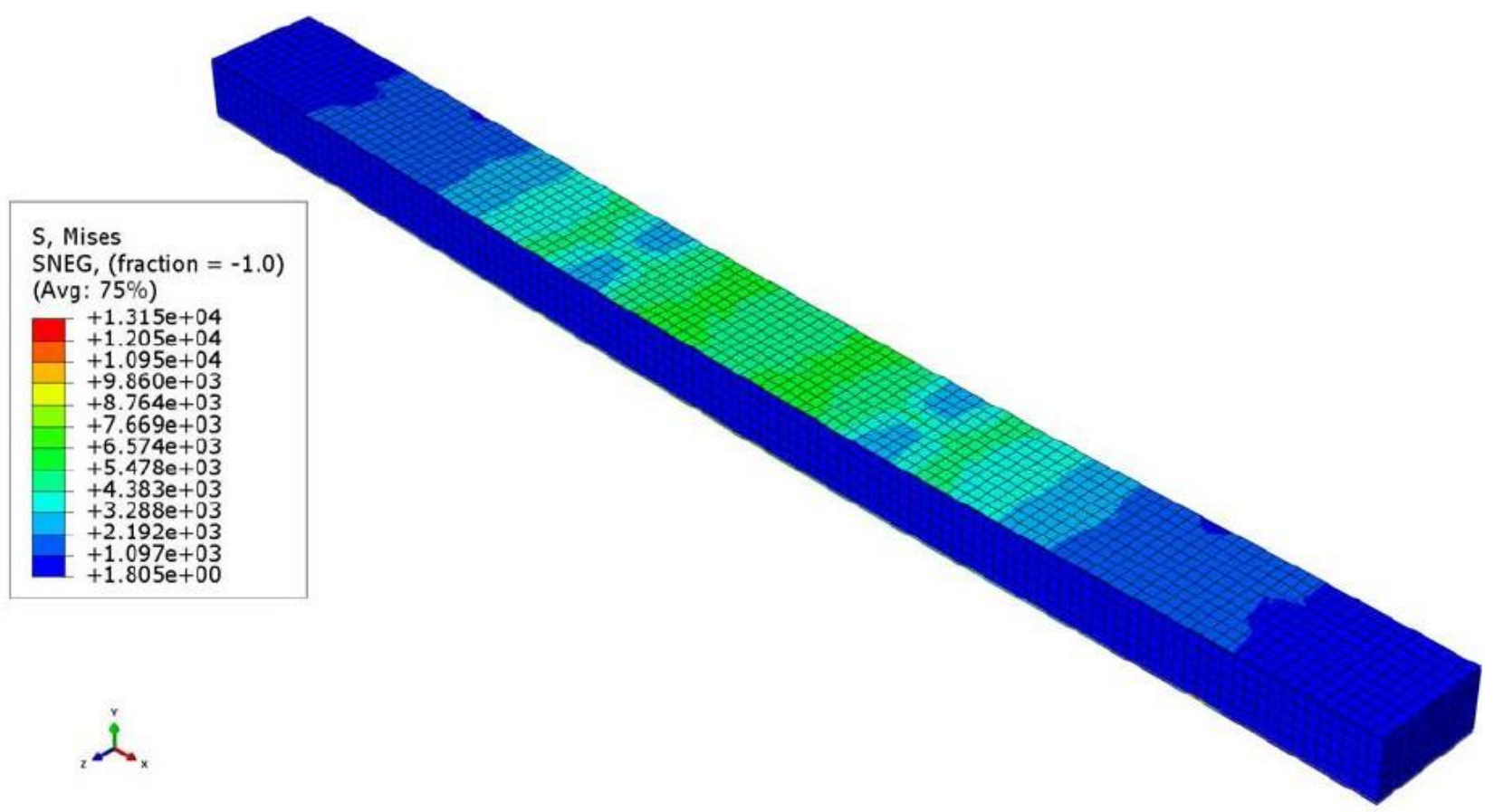

Figure 6. Von Mises delaminated beam with longitudinal DADs 